\section{Pacific Northwest}

National Laboratory

Operated by Battelle for the

U.S. Department of Energy

\title{
The Majorana Zero-Neutrino Double-Beta Decay Experiment
}

\author{
R. Gaitskell \\ A. Barabash \\ S. Konovalov \\ V. Stekhanov \\ V. Umatov \\ V. Brudanin \\ S. Egorov \\ J. Webb \\ H.S. Miley \\ C.E. Aalseth \\ D.N. Anderson \\ T.W. Bowyer \\ R.L. Brodzinski \\ D.V. Jordan
}

\author{
R.T. Kouzes \\ L.E. Smith \\ R.C. Thompson \\ R.A. Warner \\ W. Tornow \\ A. Young \\ J.I. Collar \\ F.T. Avignone \\ J.M Palms \\ P.J. Doe \\ S.R. Elliott \\ K. Kazkaz \\ H. Robertson \\ J. Wilkerson
}

February 2002

Prepared for the U.S. Department of Energy under Contract DE-AC06-76RL01830 


\title{
DISCLAIMER
}

This report was prepared as an account of work sponsored by an agency of the United States Government. Neither the United States Government nor any agency thereof, nor Battelle Memorial Institute, nor any of their employees, makes any warranty, express or implied, or assumes any legal liability or responsibility for the accuracy, completeness, or usefulness of any information, apparatus, product, or process disclosed, or represents that its use would not infringe privately owned rights. Reference herein to any specific commercial product, process, or service by trade name, trademark, manufacturer, or otherwise does not necessarily constitute or imply its endorsement, recommendation, or favoring by the United States Government or any agency thereof, or Battelle Memorial Institute. The views and opinions of authors expressed herein do not necessarily state or reflect those of the United States Government or any agency thereof.

\author{
PACIFIC NORTHWEST NATIONAL LABORATORY \\ operated by \\ BATTELLE \\ for the \\ UNITED STATES DEPARTMENT OF ENERGY \\ under Contract DE-AC06-76RL01830
}

Ty 


\section{The Majorana Zero-Neutrino Double-Beta Decay Experiment}

The Majorana Collaboration:

Brown University, Providence, RI

Institute for Theoretical and Experimental Physics, Moscow, Russia

Joint Institute for Nuclear Research, Dubna, Russia

New Mexico State University, Las Cruces, NM

Pacific Northwest National Laboratory, Richland, WA

Triangle Universities Nuclear Laboratory, Durham, NC

University of Chicago, Chicago, IL

University of South Carolina, Columbia, SC

University of Washington, Seattle, WA

February 20th, 2002

Pacific Northwest National Laboratory

Richland, WA 99352 


\begin{abstract}
The goal of the Majorana Experiment is to determine the effective Majorana mass of the electron neutrino. Detection of the neutrino mass implied by oscillation results is within our grasp. This exciting physics goal is best pursued using double-beta decay of germanium because of the historical and emerging advances in eliminating competing signals from radioactive backgrounds. The Majorana Experiment will consist of a large mass of ${ }^{76} \mathrm{Ge}$ in the form of high-resolution detectors deep underground, searching for a sharp peak at the $\beta \beta$ endpoint. If found, this peak would quantify the $T_{1 / 2}(0 v \beta \beta)$ and hence the electron neutrino mass. We present here an overview of the entire project in order to help put in perspective the scope, the level of technical risk, and the readiness of the Collaboration to begin the undertaking.
\end{abstract}

\title{
The Majorana Collaboration
}

Brown University, Providence, $R I$

Rick Gaitskell

Institute for Theoretical and Experimental Physics, Moscow, Russia

A. Barabash, S. Konovalov, V. Stekhanov, V. Umatov

Joint Institute for Nuclear Research, Dubna, Russia

V. Brudanin, S. Egorov, O. Kochetov, V. Sandukovsky

New Mexico State University

Joel Webb

Pacific Northwest National Laboratory, Richland, WA

Harry Miley, Project Coordinator, Spokesperson for DOE

Craig Aalseth, Dale Anderson, Theodore Bowyer, Ronald Brodzinski, David Jordan, Richard Kouzes, William Pitts, L. Eric Smith, Robert Thompson, Ray Warner

Triangle Universities Nuclear Laboratory, Durham, NC

Werner Tornow, Albert Young

University of Chicago, Chicago, IL

Juan Collar

University of South Carolina, Columbia, SC

Frank Avignone, Spokesperson for NSF

John M. Palms

University of Washington, Seattle, WA

Peter Doe, Steve Elliott, Kareem Kazkaz, Hamish Robertson, John Wilkerson

Draft

Page ii 


\section{Outline}

THE MAJORANA ZERO-NEUTRINO DOUBLE-BETA DECAY EXPERIMENT ..............................

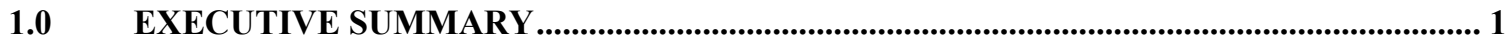

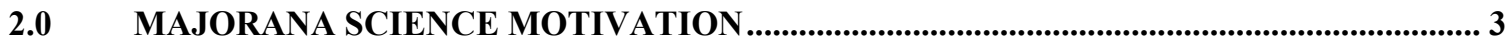

3.0 IMPLEMENTATION OF MAJORANA PROJECT APPROACH....................................... 21

4.0 PROPOSED MAJORANA R\&D PROGRAM (PHASES 1 AND 2) ....................................... 65

5.0 PROJECT MANAGEMENT PLAN ........................................................................................ 69

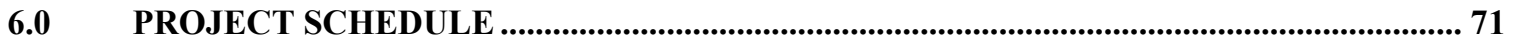

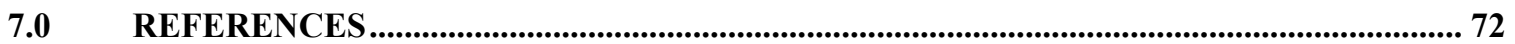

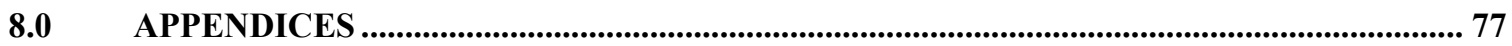

9.0 INVESTIGATOR BIOS / CURRENT AND PENDING SUPPORT ....................................... 85 


\subsection{Executive Summary}

\subsection{Purpose of Experiment}

The goal of the Majorana experiment is to determine the effective Majorana mass of the electron neutrino. Detection of the neutrino mass implied by oscillation results is now technically within our grasp. This exciting physics goal is best pursued using the wellestablished technique of searching for the neutrinoless double-beta $(0 v \beta \beta)$ decay of ${ }^{76} \mathrm{Ge}$, augmented with recent advances in signal processing and detector design. The Majorana experiment will consist of a large mass of ${ }^{76} \mathrm{Ge}$ in the form of high-resolution detectors located deep underground within a low-background environment. Observation of a sharp peak at the $\beta \beta$ endpoint will quantify the $0 v \beta \beta$-decay half-life and thus the effective Majorana mass of the electron neutrino. In addition to the modest R\&D program, we present here an overview of the entire project in order to help put in perspective the scope, the level of technical risk, and the readiness of the Collaboration to begin the undertaking.

\subsection{Phased Concept}

The Majorana Collaboration has created a three-phased approach to the work. This allows realistic shorter-term, intermediate goals, both for physics measurements and for technical accomplishments. Interim goals allow students to work within reasonable graduate degree schedules. All three phases will be carried out in a deep underground facility and tangible physics results will be obtained from each of two introductory phases. In addition, the phased approach will allow the sponsor to gauge progress, and provide a series of modest investments with reasonable payoffs.

The phases have been defined along the main technical lines of the overall project.

- Phase 1: Development of signal processing techniques for segmented crystals.

- Phase 2: Develop packaging for multiple crystals sharing a single cooling system.

- Phase 3: Including all the above plus a large quantity of enriched materials.

In Phase 1, the low background and specialized signal processing is expected to produce an interesting dark matter result in only a few months of counting. After this initial goal, results such as the verification of the two-neutrino half-life of ${ }^{76} \mathrm{Ge}$ can be achieved (values from previous experiments vary considerably).

Phase 2 consists of an array of 18 detectors. All detectors will make use of pulse-shape analysis and some fraction of the detectors will be segmented. This arrangement should provide excellent sensitivity for study of double-beta decay to excited states in ${ }^{76} \mathrm{Ge},{ }^{82} \mathrm{Se}$, ${ }^{96} \mathrm{Zr},{ }^{100} \mathrm{Mo},{ }^{130} \mathrm{Te}$, and ${ }^{150} \mathrm{Nd}$. Further improvement in dark matter sensitivity is also expected. Once the physics goals of Phase 2 are complete, the apparatus may be used for screening materials for Phase 3 or other underground experiments. In addition it may be used for ultra-trace environmental radiological measurements. The combination of low background and special signal processing would make this arrangement among the most sensitive and selective available for sample counting. 
Phase 3 will consist of 210 crystals of enriched ${ }^{76} \mathrm{Ge}$ arranged in 10 modules. All crystals will be segmented and instrumented for pulse-shape analysis. This modular arrangement results in a small footprint and allows easy access to modules. Although investigation of alternative cooling is under consideration, the baseline plan is to cool the germanium using well-understood conventional techniques. More than 20 years of double-beta decay experience and the lessons learned from the initial phases ensure that the instrumentation, analysis techniques, and packaging will be proven and the engineering risk will be minimal.

\subsection{Anticipated Sensitivity}

The Majorana Collaboration is actively refining estimates of the ultimate sensitivity of the experiment. However, the original and conservative estimation method, based on early $\mathrm{IGEX}^{+}$data, predicts an achievable $0 v \beta \beta$-decay half-life limit of $\sim 4 \times 10^{27} \mathrm{y}$, compared to a current limit of $1.9 \times 10^{25}$ y from the Heidelberg-Moscow collaboration. Depending on the nuclear matrix elements chosen, the effective neutrino mass sensitivity becomes $\left\langle\mathrm{m}_{\mathrm{v}}>=0.02-0.07 \mathrm{eV}\right.$ which is within the range implied by recent neutrino oscillation experiments. This sensitivity is a factor of 15 times better than previous work and within a factor of 2 of the sensitivity of an experiment having zero background.

\subsection{Major Requirements}

The required sensitivity can be reached with $5000 \mathrm{~kg}$-y of data from germanium enriched to $85 \%$ in ${ }^{76} \mathrm{Ge}$ operated with backgrounds lower than those obtained in the IGEX experiment. Thus a deep underground location, an active veto system, and carefullydesigned shielding are required. To lower and reject backgrounds, special signal processing techniques, detector segmentation, and underground material preparation and fabrication will be needed. Specialized techniques have been developed to utilize pulse shape and crystal segmentation data.

\subsection{Basic Timeline}

The Majorana Collaboration proposes that intensive work to complete the Phase 1 and Phase 2 instruments continue while beginning a modest campaign of germanium enrichment. Beyond the first year, the most variable schedule element in the timeline is the chosen rate of germanium enrichment. Assuming that the rate is rapidly ramped to $100 \mathrm{~kg} / \mathrm{y}, 7$ years of production will be required. Since rates of up to $200 \mathrm{~kg} / \mathrm{yr}$ are possible, this could be shortened. Our estimation and experience is that the most serious background problem is the cosmogenic production of ${ }^{68} \mathrm{Ge}$ and ${ }^{60} \mathrm{Co}$ in the ${ }^{76} \mathrm{Ge}$.

\subsection{Current Status}

Through Collaboration pooling of resources, our first (enriched) segmented detector for Phase 1 is under preparation. For Phase 2, the 16 outer crystals are in hand and the special cryostat is under construction. We are developing space underground for Phase 1 and Phase 2, as well as designing the data sharing and data hosting needed for a geographically-dispersed collaboration. The national enthusiasm for developing underground science facilities and performing underground science in the United States has lent additional momentum to our collaborative effort.

\footnotetext{
${ }^{+}$The International Germanium Experiment (IGEX) amassed 117 mole-yr of data using ${ }^{76}$ Ge detectors.
} 


\subsection{Majorana Science Motivation}

The neutrino mass range of interest favored by the results of neutrino oscillation experiments is now within the grasp of a well-designed germanium $0 v \beta \beta$-decay experiment. This well established technique has been augmented by the availability of enrichment facilities, dramatic improvements in germanium spectroscopy, and new US underground initiatives. The realization that the technology is available to achieve such a fundamental physics goal provides the basic motivation for the Majorana experiment.

To convey the importance of neutrino mass and of the Majorana experiment, we present a theoretical motivation and a brief recapitulation of past double-beta decay experiments, drawing heavily on our own completed work. We present our new technological capabilities to show how the Majorana Collaboration is positioned to make rapid strides toward neutrino mass discovery, as well as impacting other science areas.

\subsection{Theoretical Motivation of ${ }^{76} \mathrm{Ge} 0 v$ Double-Beta Decay}

Ordinary beta decay of many even-even nuclei is energetically forbidden. However, a process in which a nucleus increases its atomic number $(Z)$ by two while simultaneously emitting two beta particles is possible for some of these nuclei. Such a process is called double-beta decay. Two-neutrino double-beta decay $(2 v \beta \beta)$, defined by

$$
{ }^{Z} A \Rightarrow{ }^{Z+2} A+2 e^{-}+2 \bar{v}_{e}
$$

is an allowed second-order weak process that occurs in nature, although its rate is extremely low; half-lives for these decay modes have been measured at $\sim 10^{20}$ years or more.

A more interesting process is zero-neutrino double-beta decay $(0 v \beta \beta)$,

$$
{ }^{Z} A \Rightarrow{ }^{Z+2} A+2 e^{-}
$$

where there are no neutrinos emitted in the decay process. It is clear from this equation that unlike $2 v \beta \beta, 0 v \beta \beta$ violates lepton number conservation and hence requires physics beyond the standard model. One can visualize $0 v \beta \beta$ as an exchange of a virtual neutrino between two neutrons within the nucleus. According to the standard model of weak interactions, the first neutron emits a right-handed anti-neutrino. However, the second neutron requires the absorption of a left-handed neutrino. In order for this to happen, the neutrino would have to be massive so that it is not in a pure helicity state, and the neutrino and anti-neutrino would have to be indistinguishable. That is, the neutrino would have to be a massive Majorana particle. The Dirac or Majorana nature of the neutrino is an important open question. Neutrinoless double-beta decay is the only known practical way to determine if neutrinos are Majorana particles.

Neutrinoless double-beta decay has also been hypothesized to be driven by a number of other mechanisms: intrinsic right-handed currents, the emission of Goldstone bosons 
(Majorons), and the exchange of supersymmetric particles. However, only the process involving Majorana neutrino mass will be discussed here.

The results of atmospheric and solar neutrino oscillation experiments indicate that neutrinos have mass. This in itself excites interest in $0 v \beta \beta$ experiments. However, neutrino oscillation experiments only measure the difference in the squares of the masses of the mass eigenstates. Therefore, they indicate only the relative mass scale of the neutrinos. Even so, these experiments show that at least one neutrino has a mass greater than $\sim 50 \mathrm{meV}$. As a result, measurements of the absolute mass scale on this order are extremely exciting. Neutrinoless double-beta decay experiments are the only proposed method of measuring neutrino mass that have the potential to reach this interesting level of sensitivity.

The decay rate for this process can be written as follows:

$$
\lambda_{\beta \beta}^{0 v}=G^{0 v}\left(E_{0}, Z\right)\left\langle m_{v}\right\rangle^{2}\left|M_{f}^{0 v}-\left(g_{A} / g_{V}\right)^{2} M_{G T}^{0 v}\right|^{2} .
$$

In Equation 1, $G^{0 v}$ is the two-body phase-space factor including coupling constants; $M_{f}^{0 v}$ and $M_{G T}^{0 v}$ are the Fermi and Gamow-Teller nuclear matrix elements, respectively; and $g_{A}$ and $g_{V}$ are the axial-vector and relative vector weak coupling constants, respectively. The quantity $\left\langle m_{v}\right\rangle$ is the effective Majorana electron neutrino mass given by:

$$
\left\langle m_{v}\right\rangle \equiv\left|\sum_{k=1}^{2 n} \lambda_{k}^{C P}\left(U_{e k}^{L}\right)^{2} m_{k}\right|,
$$

where $\lambda_{k}^{C P}$ is the CP eigenvalue associated with the $\mathrm{k}^{\text {th }}$ neutrino mass eigenstate $( \pm 1$ for $\mathrm{CP}$ conservation); $U_{e k}^{L}$ is the $\mathrm{n} \times \mathrm{n}$-dimensional $(\mathrm{e}, \mathrm{k})$ matrix element of the transformation between flavor eigenstates $\left|v_{\lambda}\right\rangle$ and mass eigenstates $\left|v_{k}\right\rangle$ for left-handed neutrinos;

$$
\left|v_{\lambda}\right\rangle=\sum U_{\lambda k}\left|v_{k}\right\rangle
$$

and $m_{k}$ is the mass of the $\mathrm{k}^{\text {th }}$ neutrino mass eigenstate.

The effective Majorana neutrino mass, $\left\langle m_{v}\right\rangle$, is directly derivable from the measured half-life of the decay as follows:

$$
\left\langle m_{v}\right\rangle=m_{e}\left(F_{N} T_{1 / 2}^{0 v}\right)^{-1 / 2} \mathrm{eV},
$$

where $F_{N} \equiv G^{0 v}\left|M_{f}^{0 v}-\left(g_{A} / g_{V}\right)^{2} M_{G T}^{0 v}\right|^{2}$, and $m_{e}$ is the electron mass. This quantity derives from nuclear structure calculations and is model dependent.

The SuperKamiokande (SK) data[Sob01] imply maximal mixing of $v_{\mu}$ with $v_{\tau}$ with $\delta \mathrm{m}^{2}($ atmospheric $) \cong(55 \mathrm{meV}) \cdot$ The solar neutrino data from SK and from the Sudbury Neutrino Observatory (SNO) also imply that the large-mixing-angle solution to the solar neutrino problem is favored[Ahm01], so that $\delta \mathrm{m}^{2}($ solar $) \cong(7 \mathrm{meV})^{2}$ with $\sin ^{2} 2 \theta \cong 0.8$. 
Based on these interpretations, one probable scenario for the neutrino mixing matrix can be approximated by the following bimaximal form:

$$
\left(\begin{array}{c}
v_{e} \\
v_{\mu} \\
v_{\tau}
\end{array}\right)=\left(\begin{array}{ccc}
1 / \sqrt{2} & 1 / \sqrt{2} & 0 \\
-1 / 2 & 1 / 2 & 1 / \sqrt{2} \\
1 / 2 & -1 / 2 & 1 / \sqrt{2}
\end{array}\right)\left(\begin{array}{c}
v_{1} \\
v_{2} \\
v_{3}
\end{array}\right) \text {. }
$$

The neutrino masses can be arranged as $\delta m_{31}^{2} \approx \delta m_{32}^{2} \approx(55 \mathrm{meV})^{2}$ and $\delta m_{21}^{2} \approx(7 \mathrm{meV})^{2}$. With the available data, it is not possible to determine which hierarchy, $m_{3}>m_{1}\left(m_{2}\right)$ or $m_{1}\left(m_{2}\right)>m_{3}$, is the correct one, nor the absolute value of any of the mass eigenstates. The consideration of reactor neutrino and atmospheric neutrino data together strongly indicates that the atmospheric neutrino oscillations are dominantly $v_{\mu} \rightarrow v_{\tau}\left(\bar{v}_{\mu} \rightarrow \bar{v}_{\tau}\right)$, which implies, as seen from Eq. 5, that $v_{e}$ is a mixture of $v_{1}$ and $v_{2}$. In the chosen case, where $U_{e 3}=0$, Eq. 2 only contains one relative CP phase, $\varepsilon$, and reduces to:

$$
\left\langle m_{v}\right\rangle=\frac{1}{2}\left(m_{1}+\varepsilon m_{2}\right),
$$

whereas the large mixing angle solution of the solar neutrino problem implies

$$
\left(m_{2}^{2}-m_{1}^{2}\right)=(7 m e V)^{2} \text {. }
$$

Consideration of bi-maximal mixing yields four cases to be analyzed: (a) $\mathrm{m}_{1} \cong 0$, (b) $\mathrm{m}_{1}$ $>>\mathrm{meV},(\mathrm{c}) \mathrm{m}_{3} \cong 0$ and (d) the existence of a mass scale, $\mathrm{M}$, where $\mathrm{M}>>55 \mathrm{meV}$.
a) If $m_{1}=0, m_{2} \cong 7 \mathrm{meV}$, and $\left\langle m_{v}\right\rangle=\frac{m_{2}}{2}$.
b) If $m_{1}>>7 m e V \equiv \mathrm{M}$ and $\left\langle m_{v}\right\rangle \cong \frac{M}{2}(1+\varepsilon)$.
c) If $m_{3}=0, m_{1} \cong m_{2} \cong 55 \mathrm{meV}$, and $\left\langle m_{v}\right\rangle \cong 0$ or $55 \mathrm{meV}$.
d) If $M>>55 m e V, m_{1} \cong m_{2} \cong(M+55 m e V)$, and $\left\langle m_{v}\right\rangle \cong \frac{m_{1}}{2}(1+\varepsilon)$.

If we assume that $\varepsilon \cong+1$, and that neutrinos are Majorana particles, then it is very probable that $\left\langle m_{v}\right\rangle$ lies between $3.5 \mathrm{meV}$ and the present bound from ${ }^{76} \mathrm{Ge} 0 \mathrm{v} \beta \beta$ experiments.

In discussing the value of effective electron neutrino Majorana mass, $\left\langle m_{v}\right\rangle$, that could render neutrinoless double-beta decay observable, we chose one scenario out of a number of possibilities. There have been several extensive discussions of the various interpretations of neutrino oscillation data, and their impact on the range of probable values of this important parameter[Ahm01, Pas01, Far01, Bil01, Kla01a]. 
In one case, for example, the authors found that for three-neutrino mixing, $|\langle m\rangle| \sim 10 \mathrm{meV}$ if the neutrino mass spectrum is hierarchical[Bi199]. On the other hand, if two of the neutrino eigenstates are quasi-degenerate, with $m_{l}$ having a small mass, $|\langle m\rangle|$ could be as large as $100 \mathrm{meV}$. In this case, early stages of one of the next-generation experiments could directly observe neutrinoless double-beta decay.

Another interpretation[Kla01a] gives specific predictions for the ranges of the Majorana mass parameter for all possible solar neutrino solutions in the cases of hierarchy, partial degeneracy and inverse hierarchy. These predicted masses range from $1 \mathrm{meV}$ to $1.0 \mathrm{eV}$.

The impact on CP-violation in the neutrino sector and its connection to neutrino oscillations, tritium beta-decay, and double-beta decay experiments has been discussed by several authors[Ahm01, Pas01, Far01, Bil01, Kla01a]. Three- and four-neutrino flavor scenarios have been considered[Far01] in the context of next-generation tritium betadecay measurements and double-beta decay experiments. They discuss how these data could help determine the pattern of neutrino mass eigenstates, and possibly the relative $\mathrm{CP}$ - violating phase in the case that two neutrino states are involved in solar neutrino oscillations.

New literature in the field is appearing frequently. It almost always refers to the importance of conducting next-generation zero-neutrino double-beta decay experiments. A complete understanding of the neutrino mass matrix depends on three types of data, each analogous to one leg of a three-legged stool. They are: neutrino oscillations, tritium beta-decay measurements and neutrinoless double-beta decay. Each is necessary for a complete picture. The case for a significant investment in next-generation experiments of all three types is being made by many experts in the field.

Because double-beta decay always results in the emission of two electrons, and because these electrons travel very short distances in germanium, $0 v \beta \beta$-decay should appear as a sharply defined spectral line at the endpoint energy of the decay, or $2039 \mathrm{keV}$, in a high resolution germanium spectrometer. This energy is above most radiological backgrounds (exceptions are discussed in detail later).

This internal source technique is quite convenient for high-resolution germanium detectors: they have excellent energy resolution $(\sim 0.2 \%$ at $2039 \mathrm{keV})$ and normally run for many years without maintenance as long as they are maintained cold, dark, and in vacuum. Based on the extensive previous experience of the Majorana Collaboration, the most serious problem will be cosmogenic radionuclides in the detector crystal. Given a large mass of material, a long run time, and an attainable factor of 40 reduction in background compared to previous work, sensitivity to Majorana neutrino masses as low as $0.02-0.07 \mathrm{eV}$, and thus the mass region of interest, should be attainable. 


\subsection{Completed Double-Beta Decay Experiments}

The first laboratory search for double-beta decay was made in 1948by Fireman[Fir48]. The experiment involved a search for coincident pulses in Geiger counters in proximity to a source of ${ }^{124} \mathrm{Sn}$, and a limit of $\mathrm{T}_{1 / 2}>3 \cdot 10^{15} \mathrm{yr}$ was assigned to the decay mode. Based on the Standard Model of Physics, as understood at that time, this was assumed to be for the neutrinoless mode mediated by Majorana neutrinos, which would have been expected to have a decay rate 7-9 orders of magnitude greater than the two-neutrino mode.

The existence of double-beta decay was first confirmed in a series of geochronological experiments by Inghram and Reynolds[Ing50] in 1950 using ${ }^{130} \mathrm{Te}$. These results were confirmed by Takaoka and Ogata[Tak66] in 1966 and again by Kirsten, et al.[Kir67a] in 1967. Kirsten, Gentner, and Schaeffer [Kir67b] also reported measurement of doublebeta decay for ${ }^{82} \mathrm{Se}$ in 1967 . These experiments relied on mass-spectrometric measurements of the noble gas daughters entrained in very old ores. Excesses of ${ }^{130} \mathrm{Xe}$ and ${ }^{82} \mathrm{Kr}$ were used to determine the double-beta decay half-lives from ores that were independently dated by other techniques. While these measurements unequivocally demonstrated that double-beta decay was a real phenomenon, nothing could be inferred about the particular mode of double-beta decay responsible for the buildup of daughter products.

The ingenious utilization of a high-resolution germanium diode gamma-ray spectrometer as both the source and detector for a double-beta decay experiment was introduced by Fiorini and colleagues[Fio67] in 1967. They were able to assign a limit to the ${ }^{76} \mathrm{Ge}$ neutrinoless double-beta decay mode of $\mathrm{T}_{1 / 2}>2 \cdot 10^{20} \mathrm{yr}$.

The first direct laboratory observation of double-beta decay was reported by Elliott, Hahn, and Moe[El187] in 1987. They used a Time Projection Chamber to measure the two-neutrino double-beta events from a source consisting of $14 \mathrm{~g}$ of $97 \%$ isotopically enriched ${ }^{82}$ Se contained between thin aluminized Mylar sheets. Their value of $\mathrm{T}_{1 / 2}=$ $1.1 \times 10^{20} \mathrm{yr}$ was in excellent agreement with the geochronological half-life reported earlier for this isotope.

In 1988, Avignone and Brodzinski[Avi88] in a review article reported on the use of an isotopically-enriched germanium spectrometer by the ITEP-EREVAN group, and predicted that the combination of large isotopically-enriched germanium spectrometers coupled with application of good background-reduction practices would ultimately lead to a sensitivity for the effective electron neutrino mass of a few tens of meV. Interestingly, this prediction corresponds precisely with the now-known requisite mass range based on the atmospheric and solar oscillation results and with the mass range attainable by this proposed Majorana Collaboration experiment.

The first reported measurements of the two-neutrino half life for ${ }^{76} \mathrm{Ge}$ were made in 1990 by Vasenko, et al.[Vas90] and by Miley, et al.[Mil90], later confirmed by Avignone et al.[Avi91]. The Russian collaboration measurements were made using the isotopicallyenriched detector referred to above and were in substantial agreement with the U.S.-based 
measurement, which was determined from data acquired with two 1-kg natural isotopic detectors. The reported half lives were $\mathrm{T}_{1 / 2}=0.92 \times 10^{21} \mathrm{y}$ and $1.1 \times 10^{21} \mathrm{y}$, respectively. The confirmation experiment utilized a small detector enriched to $86 \%$ in ${ }^{76} \mathrm{Ge}$.

Additional direct measurements of double-beta decay were reported for ${ }^{100}$ Mo in 1991 by Elliott, et al.[El191] and by Ejiri, et al.[Eji91] and for ${ }^{150} \mathrm{Nd}$ in 1993 by Artem'ev et al.[Art93] and by Elliott, et al.[El193]. The virtually-identical results for the two-neutrino double-beta decay of ${ }^{100} \mathrm{Mo}$ to the ground state of ${ }^{100} \mathrm{Ru}, \mathrm{T}_{1 / 2}=1.16(1.15) \times 10^{19} \mathrm{y}$, was followed by a direct measurement of the double-beta decay of ${ }^{100}$ Mo to the first excited $0^{+}$state in ${ }^{100} \mathrm{Ru}$ by Barabash, et al.[Bar95] in 1995. The double-beta decay to the $1130.29-\mathrm{keV}$ state was observed by single-gamma measurements of the cascade deexcitation gamma-rays at 539.53 and $590.76 \mathrm{keV}$ from a $956-\mathrm{g}$ sample of $98.468 \%$ isotopically-enriched ${ }^{100}$ Mo metal powder. The resulting half-life was determined to be $\mathrm{T}_{1 / 2}=6.1 \times 10^{20} \mathrm{yr}$.

All the small scale ${ }^{76} \mathrm{Ge}$ double-beta decay experiments have now been terminated, with the current neutrinoless half-life limit $\mathrm{T}_{1 / 2}>1.9 \times 10^{25} \mathrm{y}[\mathrm{Kla} 01 \mathrm{~b}]$. This half-life corresponds to a limit for the Majorana neutrino mass of $0.3-1 \mathrm{eV}$, depending on the theoretical nuclear matrix elements chosen. To become sensitive to a neutrino mass an order of magnitude or more smaller will require a large increase in the scale of a doublebeta decay experiment; precisely the increase described in this Majorana Collaboration document.

\subsection{Ultimate Sensitivity of the Majorana Experiment}

To estimate the sensitivity of the Majorana Phase 3 experiment we begin with the published[Bro95] spectrum from an enriched germanium detector that had been operated under 4000 meters water-equivalent (mwe) shielding from cosmic rays. The components of the background were computed based on the use of validated spallation mechanisms and rates. The computed rate at the region of interest $\left(\mathrm{R}_{\mathrm{c}}=0.29 \mathrm{cts} / \mathrm{keV} / \mathrm{kg} / \mathrm{yr}\right)$ from the spallation isotopes actually exceeded the experimentally measured count rate $\left(R_{e}=0.1\right.$ $\mathrm{cts} / \mathrm{keV} / \mathrm{kg} / \mathrm{yr}$ ). Therefore a conservative estimate of $0.2 \mathrm{cts} / \mathrm{keV} / \mathrm{kg} / \mathrm{yr}$ has been taken as an intermediate value. In practice, lower values may be possible by keeping high-energy neutrons away from the raw enriched material and by fabricating the detector crystal underground.

We wish to scale the count rate of the previous experiment to that of our baseline plan: a $500 \mathrm{~kg}$ detector operated for 10 years. We correct that rate to account for the decay of activities that will occur before and during the experiment. Finally we correct the rate to account for the new technologies that we will employ.

The detector used for these sensitivity estimates had been zone refined, so that the ${ }^{60} \mathrm{Co}$ $\left(\mathrm{T}_{1 / 2}=5.2 \mathrm{y}\right)$ inside the crystal, created by cosmic-ray generated neutrons, was expected to be low. But the detector had been above ground long enough before zone refining to have reached equilibrium with respect to ${ }^{68} \mathrm{Ge}\left(\mathrm{T}_{1 / 2}=271 \mathrm{~d}\right)$, another important internal contaminant. The first reduction in this background rate comes from decay during the 
construction period. This has been calculated using a modest rate of enrichment and assuming quarterly shipments of enriched material during the construction period.

Decay during the construction period would decrease the ${ }^{68} \mathrm{Ge}$ by an average factor of 0.24 , and an additional factor of 0.11 during the data acquisition of the experiment. Similarly, ${ }^{60}$ Co would decay during construction to reduce the count rate to 0.73 of the original rate by the experiment start, and during the 10 year data acquisition the average rate during the experiment would be 0.55 times that at the beginning.

Table 2-1. Estimation of sources of activity in early IGEX data

\begin{tabular}{cccccccc}
\hline $\begin{array}{c}\text { Spallation } \\
\text { Isotope }\end{array}$ & $\mathrm{T}_{1 / 2}(\mathrm{~d})$ & $\begin{array}{c}\text { Rate from } \\
\text { [Bro95] }\end{array}$ & $\begin{array}{c}\text { After } \\
\text { Construction }\end{array}$ & $\begin{array}{c}\text { Rate During } \\
\text { Experiment }\end{array}$ & $\begin{array}{c}\text { Total in } \\
\text { ROI }\end{array}$ & $\begin{array}{c}\text { After PSD } \\
\text { Rejection }\end{array}$ & $\begin{array}{c}\text { After Seg } \\
\text { Rejection }\end{array}$ \\
\hline${ }^{68} \mathrm{Ge}$ & 270.82 & 0.1562 & 0.03702 & $3.93 \mathrm{E}-03$ & 70.15 & 18.59 & 2.57 \\
${ }^{56} \mathrm{Co}$ & 77.27 & 0.0238 & 0.00212 & $6.43 \mathrm{E}-05$ & 1.15 & 0.30 & 0.04 \\
${ }^{60} \mathrm{Co}$ & 1925.2 & 0.0177 & 0.01294 & $7.15 \mathrm{E}-03$ & 127.55 & 33.80 & 4.66 \\
${ }^{58} \mathrm{Co}$ & 70.82 & 0.0024 & 0.000202 & $5.60 \mathrm{E}-06$ & 0.10 & 0.03 & 0.00 \\
& & cts $/ \mathrm{keV} / \mathrm{kg} / \mathrm{y}$ & $\mathrm{cts} / \mathrm{keV} / \mathrm{kg} / \mathrm{y}$ & $\mathrm{cts} / \mathrm{keV} / \mathrm{kg} / \mathrm{y}$ & Counts & Counts & Counts \\
\hline Total & & 0.2 & 0.0523 & 0.0112 & 198.95 & 52.72 & 7.28 \\
\hline
\end{tabular}

Thus, accounting for decay, we would take the average background rate during the experiment to be $0.01 \mathrm{cts} / \mathrm{keV} / \mathrm{kg} / \mathrm{yr}$. Thus the effect of pre-deployment decay is effectively a reduction of $94 \%$ or a factor of 17.8 .

The number of ${ }^{76} \mathrm{Ge}$ atoms in $500 \mathrm{~kg}$ of enriched germanium $\left(85 \%{ }^{76} \mathrm{Ge}\right)$ is $\mathrm{N}=$ $3.429 \times 10^{27}$. An energy window of $\delta \mathrm{E}=3.568 \mathrm{keV}$ is expected to capture $83.8 \%$ of the events in a sharp peak at $2039 \mathrm{keV}$. If $\mathrm{B}=\mathrm{b} * \delta \mathrm{E} * \mathrm{~N} * \delta \mathrm{t}$ and $\delta \mathrm{t}$ is 10 years, we would expect to observe 199 background counts.

The next step in estimating the sensitivity of the experiment is to apply two new but easily implemented techniques. The first is an experimentally-demonstrated technique to measure the multiplicity of energy depositions by analyzing digitized current pulses using a robust, self-calibrating technique. This method has been shown to accept $\varepsilon_{\mathrm{PSD}}=80.2 \%$ of single site pulses (like double-beta decay) and to reject $73.5 \%$ of background. The second technique involves the electrical segmentation of the detector crystal to form many small segments within a crystal. A simple Monte Carlo analysis of this configuration was carried out only to count the segments with significant energy deposition and reject events with a multiplicity $>1$. This cut accepted $\varepsilon_{\mathrm{SEG}}=90.7 \%$ of double-beta decay pulses and rejected $86.2 \%$ of backgrounds like ${ }^{60} \mathrm{Co}$ and ${ }^{68} \mathrm{Ge}$, which are highly multiple.

Applying the background reduction factors to the simple calculation above, only 7.28 counts of the original 199 counts survive in our $3.568 \mathrm{keV}$ analysis window, a reduction of $96.3 \%$ or a factor of 27.3 . 
For a positive signal at the $90 \% \mathrm{CL}$, we would then need to observe 11 counts $\left(\mathrm{L}_{\mathrm{c}}=11\right.$ actually yields $91.2 \% \mathrm{CL}$ ). This is an additional 3.72 counts over the expected 7.28 background events. Computing the $0 v \beta \beta$ half-life must then take into account this number of observable counts, the cut efficiencies, and the fraction of the $0 v \beta \beta$-decay peak found in the analysis window. Thus

$$
T_{1 / 2}=\frac{\ln (2) \cdot N \cdot \Delta t \cdot \varepsilon_{P S D} \cdot \varepsilon_{S E G} \cdot 83.8 \%}{3.72}=3.8 \times 10^{27} y .
$$

Our formulation for the effective Majorana mass of the electron neutrino is

$$
\left\langle m_{v}\right\rangle=\frac{m_{e}}{\sqrt{F_{N} \cdot T_{1 / 2}}}
$$

where $F_{N}$ is a set of nuclear factors adopted from various authors[Aal99a]. The variety of nuclear calculations gives a range of observable effective Majorana neutrino mass from $0.02 \mathrm{eV}$ to $0.07 \mathrm{eV}$.

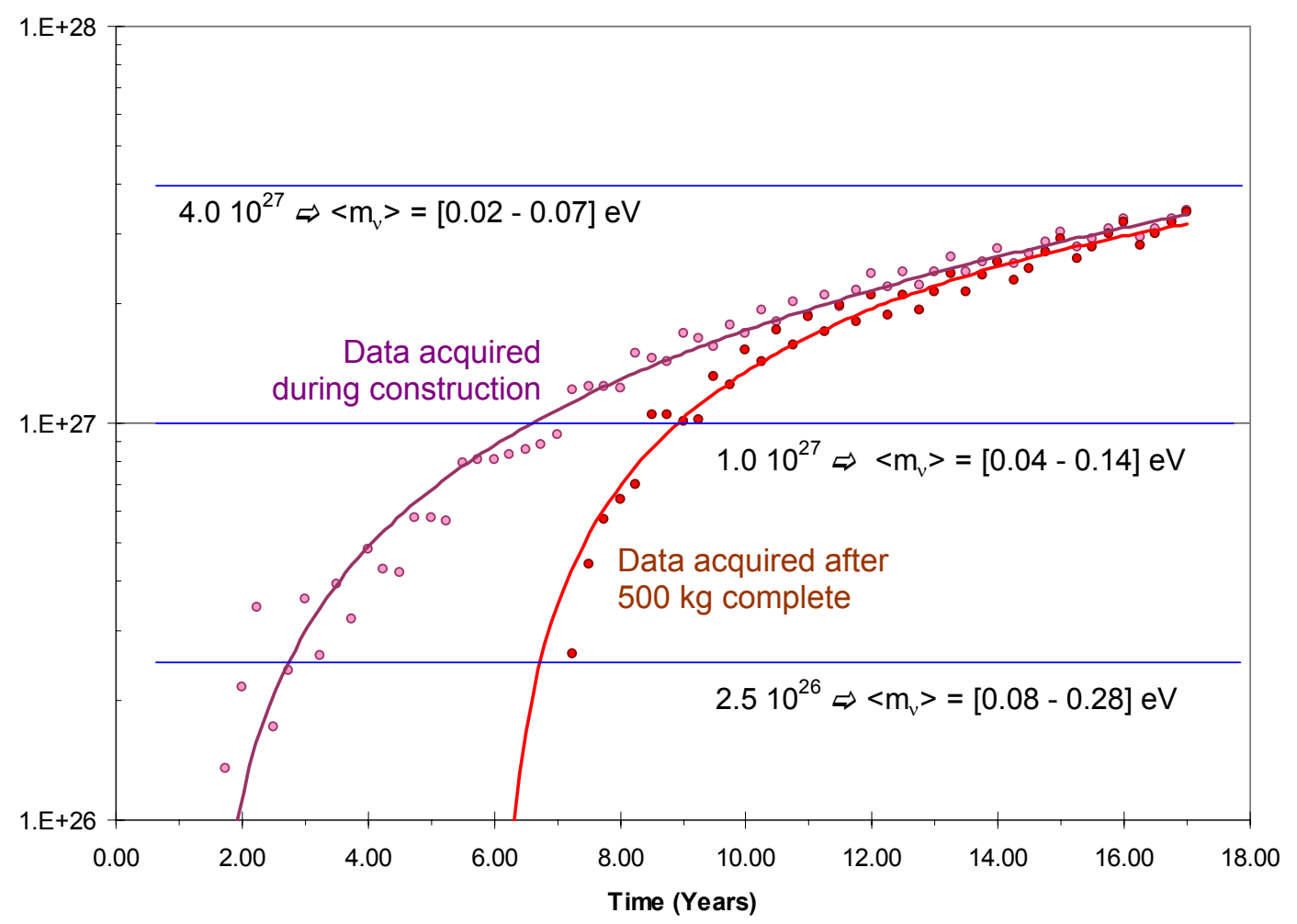

Figure 2-1 Sensitivity vs. time of the Majorana baseline plan using very conservative background assumptions. This plan assumes a gradual increase in production of germanium until $100 \mathrm{~kg} / \mathrm{yr}$ is reached for a total of $500 \mathrm{~kg}$ (Figure 6-2 below shows $200 \mathrm{~kg} / \mathrm{y}$ sensitivity vs. time estimates). Milestones in $T^{1} / 2$ are shown at $0.25,1.0$, and $4.010^{27}$ years. Rounding of $<\mathrm{m}_{v}>$ (i.e. $0.074-0.065 \mathrm{eV}$ ) leads to a $T^{1 / 2}$ target range of $3.410^{27}-4.410^{27} \mathrm{y}$.) Scatter about the trend lines is due to the integer nature of Poisson statistics. 
Many other formulations of this sensitivity calculation are possible. For instance, it is possible to calculate the expected rate of background due to cosmogenic isotopes in the crystal assuming many different scenarios producing far less initial background. This is a reasonable approach and will lead to a lower starting background. It is possible, however, to hypothesize away all backgrounds without regard to the effort involved. We chose to start with a known, reproducible starting point so that the result would be credible and attainable. The many details of the technologies involved ranging from lead bricks to multi-dimensional parametric pulse analysis are described in some detail in Section 3 below.

The calculations in this section have covered in some detail the effects of backgrounds on a $5000 \mathrm{~kg}-\mathrm{y}$ experiment in which the mass is $500 \mathrm{~kg}$ and the time is 10 years. A completely different approach would be to consider ways of reducing the time needed to complete the experiment by allowing different total masses of enriched material. In this approach, one might optimize not for lowest cost but for shortest total time to completion, including construction. Many details are not considered in this estimate, such as increased labor costs, increased detector production costs, and so forth. Figure 2-2 shows the results of a simple analysis with background rates similar to[Bro95]. Rates of enrichment above $200 \mathrm{~kg} / \mathrm{y}$ are purely hypothetical, but might be reached by employing more than one Russian enrichment facility.

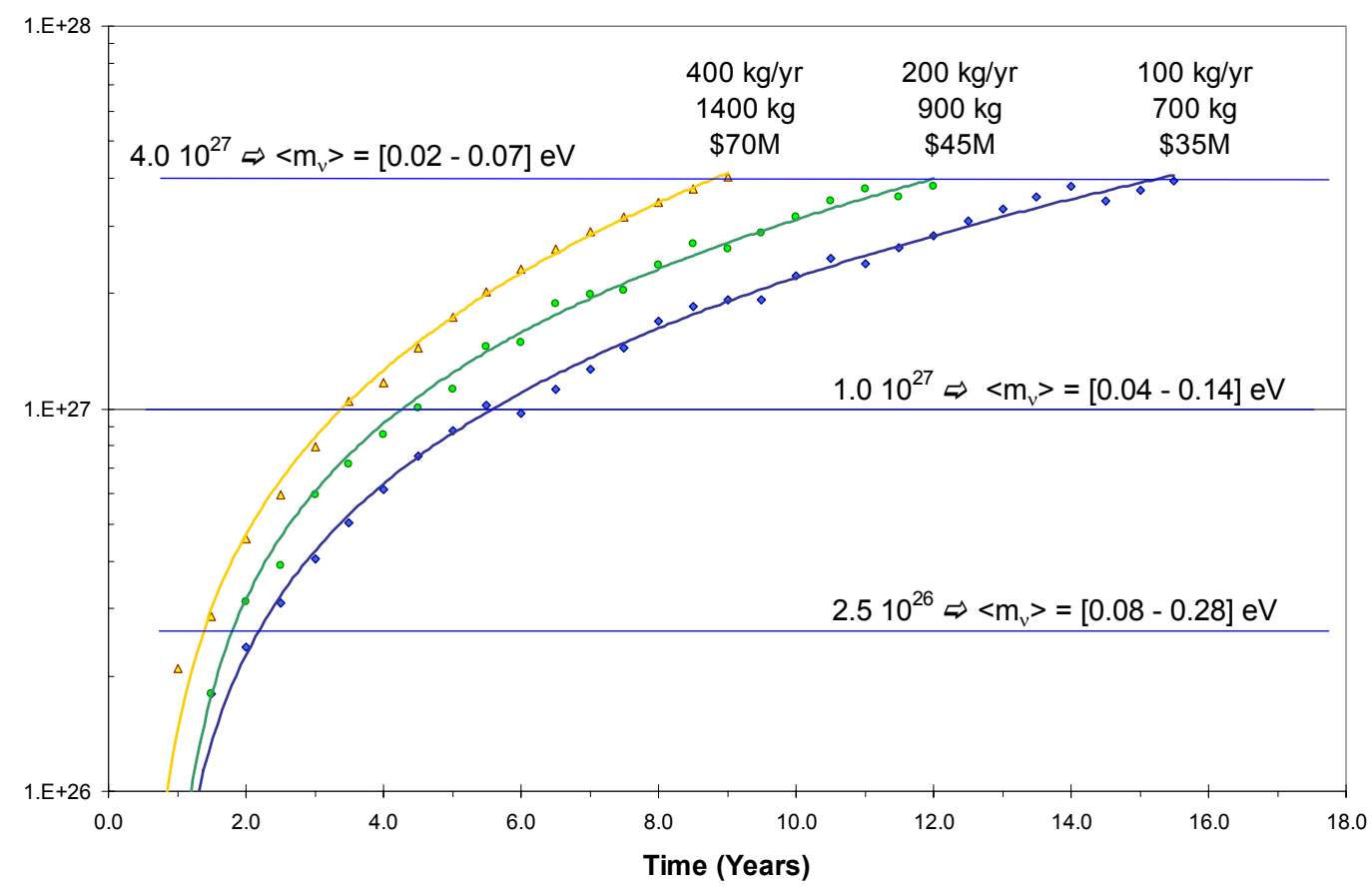

Figure 2-2 Optimizing schedule where cost is a free parameter. This assumes immediate full production vs. the gradual start of the baseline plan. All costs are enrichments only and are based on \$50/g. See Appendix 1 for more enrichment cost detail.

This simple analysis shows that a significantly reduced schedule is possible with greater investment in enrichment. 


\subsection{Other Science Applications of the Majorana Experiment}

The Majorana experiment is foremost a neutrino mass experiment. However, we will capitalize on its unique capabilities to realize other interesting physics at little added cost. The two examples discussed here are dark matter and axion searches. Care in the construction of the Majorana Phase 3 apparatus should yield significant sensitivity for both of these purposes.

\section{Other Science: Majorana as a Weakly Interacting Massive Particle detector}

Majorana should be able to contribute significantly to dark matter searches. The Majorana sensitivity should be similar and complementary to that of CDMS-II.

Extensive gravitational evidence indicates that a large fraction of the matter in the universe is non-luminous, or "dark"[Ber01]. However, the nature and quantity of the dark matter remain unknown, providing a central problem for astronomy and cosmology[Ko190, Pee93]. Recent measurements of the cosmic microwave background radiation[Pry01], as well as arguments based on Big Bang Nucleosynthesis and the growth of structure in the universe[Sre00], suggest that dark matter is predominantly made up of non-baryonic particles outside the standard model of particle physics. Supersymmetric particle physics models provide a natural candidate for dark matter: the lightest superpartner, usually taken to be a neutralino with typical mass about $100 \mathrm{GeV} / \mathrm{c}^{2}$ [Jun96, El197, Eds97, Bot00]; experimental bounds from LEP give a lower limit of $46 \mathrm{GeV} / \mathrm{c}^{2}[\mathrm{El} 100]$.

More generically, one can consider a class of Weakly Interacting Massive Particles (WIMPs)[Lee77], which were once in thermal equilibrium with the early universe, but were "cold," i.e. moving non-relativistically at the time of structure formation. Their density today is then determined roughly by their annihilation rate, with weak-scale interactions if the dark matter is mainly composed of WIMPs. WIMPs are expected to have collapsed into a roughly isothermal, spherical halo within which the visible portion of our galaxy resides, consistent with measurements of spiral galaxy rotation curves[Kol90]. Direct detection of WIMPS is possible through their elastic scattering from nuclei[Goo85,Pri88]. Calculations of the fundamental WIMP-quark cross-sections require use of a model, usually the Minimal Supersymmetric Standard Model (MSSM)[Jun97]. This interaction, summed over the quarks present in a nucleon, gives an effective WIMP-nucleon cross section. In the low momentum-transfer limit, the contributions of individual nucleons are summed coherently to yield a WIMP-nucleus cross section; these are typically smaller than $10^{-6} \mathrm{pb}[\mathrm{E} 1101 \mathrm{a}$, Ell01b, Bat01, Mat00, Bed97, Bal01, Cor00]. The nuclear recoil energy is typically few keV up to tens of $\mathrm{keV}$ [Lew96] since WIMP velocities relative to the Earth should be typical of Galactic velocities.

An ultra-low-background segmented Ge detector array designed for double-beta decay has the potential to be used for a WIMP dark matter search. In this section the factors affecting the sensitivity of such a search are summarized, highlighting the additions and complementary studies necessary to achieve this goal without compromising the primary 
$0 v \beta \beta$-decay goal. Conservative sensitivity projections are also made. It is clear that an incremental approach to improving radioactive background levels/rejection and array performance at low energies will be necessary. The results from Phases 1 and 2 will be critical in assessing the ultimate dark matter sensitivity. The expected WIMP recoil spectrum in germanium extends from threshold, $<1 \mathrm{keV}$, to $\sim 20 \mathrm{keV}$ (ionization energy), in contrast to the much higher energy $\beta \beta$-decay signature. Due to the low energy of the region of interest, additional attention must be paid to screening detector materials for their contribution to background in this window. Several attractive features that the Majorana experiment displays as a WIMP detector are listed as follows:

1) Close-packing (self-shielding) and segmentation of the crystals will contribute to reducing the gamma-ray background in the low-energy region where the WIMP signal is expected. Single isolated nuclear recoils are expected WIMP interactions, whereas gamma-rays generally interact more than once in the detector ensemble, allowing them to to be rejected in a large, spatially-divided device like Majorana Phase 3. Phase 1 of the Majorana experiment will allow these background rejections capabilities to be better characterized.

2) Segmentation also lowers detector capacitance, reducing the energy threshold and increasing the acceptance of the WIMP signal. Thresholds as low as $0.75 \mathrm{keV}$ are achieved in segmented HPGe, a considerable reduction from a customary $5-10 \mathrm{keV}$ in unsegmented large diodes.

3) The spatial information revealed by pulse-shape analysis (PSA) may help eliminate surface events such as low-to-medium energy betas or other surface contamination, already a limiting background in some WIMP detectors[Kud01]. The feasibility of this approach and its relevance to this detector application must be studied in depth during Phase 1: no attempt to exploit PSA in the low-energy region has been made by this collaboration yet.

4) Majorana's ability to reject low-energy neutron events is less evident but potentially important. In a typical deep underground location the dominant neutron flux arises from $(\alpha, n)$ and natural fission in rock, and to a lesser extent from hard neutrons originating in muon spallation in rock and shielding. The main concern here is from neutrons with energies above $\sim 200 \mathrm{keV}$ and a typical flux $\sim 10^{-6} \mathrm{n} / \mathrm{cm}^{2} / \mathrm{s}[\mathrm{Be} 189$, Ste01]. The referenced energy spectrum dies off rapidly above $\sim 5 \mathrm{MeV}$. The maximum recoil energy imparted by a neutron to a Ge nucleus is $\sim 1 / 18$ of the incident energy, with only a few percent going into ionization, the rest being lost to phonons. This causes the neutron recoil signal to concentrate below $\sim 60 \mathrm{keV}$ ionization energy. Neutron recoils are identical to those expected from WIMPs. They constitute the limiting background in any WIMP detector, unless a rejection method or substantial neutron shielding can be applied.

An estimate shows that the present low energy signal in IGEX detectors $(0.05$ counts $/ \mathrm{keV} / \mathrm{kg} /$ day) is indeed compatible with an origin in neutron-induced recoils. This same observation that neutron recoils may already be limiting WIMP searches has been recently emphasized by the EDELWEISS collaboration[Ste01]. The viability of using 
additional external shielding in Majorana (neutron moderator and active muon veto) without affecting $\beta \beta$ performance, or physical access to the detectors, will be studied: A full GEANT geometry is under development. Experimental data from Phase 1 will be used to validate the simulation, which can then guide the final shielding structure.

Energetic (50-600 MeV) "punch-through" neutrons generated by cosmic-ray interactions in surrounding rock can easily penetrate traditional moderator shielding. This source of neutrons can be reduced by locating the experiment at a deep site[Gai01]. However, a significant veto against neutron hits can be achieved in Majorana by monitoring event multiplicity. Considering that the mean free path between recoils in Ge for the neutron energies of concern is $\sim 5 \mathrm{~cm}$, the finely grained segmentation and close packing of Majorana detectors should allow to identify a large fraction of neutron events by their characteristic multiple-site interactions. This promising feature of Majorana merits a dedicated Monte Carlo analysis. CDMS-I relies on this same consideration to tag neutron events[Abu00]. Majorana should exhibit a better neutron rejection ability from its larger target mass.

Another worthy advantage of Majorana as a WIMP detector is the large exposure to be collected. For an apparatus like this, with a planned $500 \mathrm{~kg}$ target mass and 10-year data collection, the best WIMP sensitivity originates not from the standard signal-to-noise analysis method (i.e., comparing the expected WIMP signal in a spectral region with the background by means of a suitable statistical estimator), but from an absence of temporal modulations in the background that could otherwise be assigned to a time-dependent WIMP signal. A known example is the yearly modulation in scattering rate and deposited energy expected from the combined movement of Earth and Sun through an isotropic WIMP galactic halo[Dru86].

The improved sensitivity in the modulation analysis is brought about by the progressive reduction in statistical background fluctuations that comes with an increasing exposure. Several authors have discussed this approach to data analysis[Ceb01]. A stable detector gain over long periods of time (years) is a necessary condition for its applicability. In the case of unsegmented HPGe this has been already demonstrated for periods of $\sim 2$ years[Dru92]. It is nevertheless our goal to corroborate this crucial point during Phases 1 and 2 using segmented devices and Majorana's DAQ system. This system will ultimately be designed to monitor detector acceptance stability directly, at the $\sim 0.1 \%$ level for the low-energy bins.

For the time being, a first Monte Carlo calculation of the minimum detectable modulated background fraction after a 5,000 kg-y exposure has been performed, using the statistical estimator proposed by Freese[Fre92]. In order to obtain sensitivity projections from this Monte Carlo it is necessary to make a working hypothesis about Majorana's achievable background in the energy region between a few $\mathrm{keV}$ and a few tens of $\mathrm{keV}$. In the interim until Phase 1 background measurements and dedicated Monte Carlo simulations are completed, a flat 0.005 counts $/ \mathrm{keV} / \mathrm{kg} /$ day from detector threshold up to $20 \mathrm{keV}$ ionization energy is assumed. It must be emphasized that this represents just one order of magnitude improvement with respect to the most recent IGEX data. This is believed to be 
a conservative premise in view of the anticipated background rejection capabilities discussed above. The contribution from radioactive cosmogenic activation products to this energy region is much lower than the background assumed, even for a relatively long $\sim 30$ day crystal exposure at sea level[Bau01], leaving ample room for improvements. Furthermore, cosmogenic background rates for natural Ge[Avi92, Co192] should be taken as a conservative upper limit for Majorana: Activation rates for ${ }^{76} \mathrm{Ge}$ are roughly one order of magnitude smaller due to the higher neutron spallation-reaction energy thresholds[Col00], with a possible exception for tritium production. This represents a clear advantage vis-à-vis other large-mass WIMP detectors planning to use natural Ge, e.g., the GENIUS test facility.

Even with this conservative approach, the expected sensitivity via annual modulation analysis approaches CDMS-II projections (Fig. 2-1) after collection of the planned 5,000 $\mathrm{kg}$-y exposure, if a threshold $\sim 1 \mathrm{keV}$ can be achieved. In addition, if the neutralino scattering cross-section resides close to the limit of sensitivity for both experiments, $\sim 10^{-8}$ pb (Fig. 2-1), Majorana may detect the annual modulation signature, something that the much smaller CDMS-II future total exposure $(\sim 10 \mathrm{~kg}-\mathrm{y})$ disallows. CDMS expects to

\section{Majorana dark matter sensitivity similar to and complementary with CDMS-II}

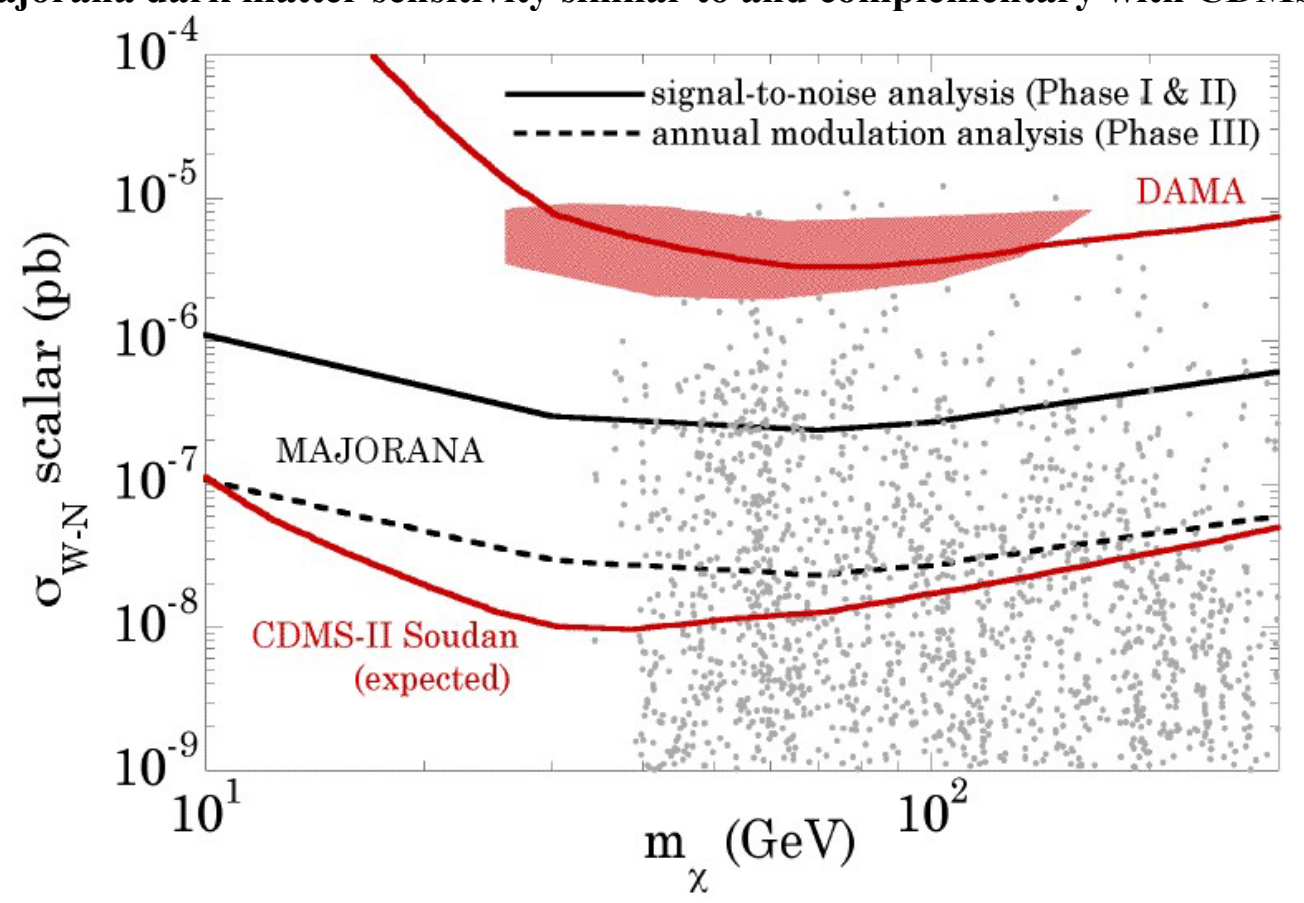

Figure 2-3 Projected 95\% C.L. Majorana WIMP limits for an assumed low-energy background of 0.005 counts $/ \mathrm{keV} / \mathrm{kg} / \mathrm{day}$, just one order of magnitude lower than in present unsegmented single HPGe detectors. Calculated for an ionization energy threshold of $1 \mathrm{keV}$, achievable via segmentation. "Signal-to-noise" limits are within reach after modest exposures $<1$ kg-y (i.e., during phases 1-2 of the project). "Annual modulation" limits are calculated for the total exposure of $5000 \mathrm{~kg}-\mathrm{y}$. Present DAMA limits and expected CDMS limits are offered as a reference. The shaded region is presently favored by DAMA to explain an unconfirmed WIMP annual modulation in its signal. Dots represent the location in this phase space (spin-independent scattering cross section vs. WIMP mass) of plausible supersymmetric neutralino WIMP candidates, using the same parameters as in [Col00]. Even under these very conservative background assumptions, the expected WIMP Majorana sensitivity is comparable to the most promising cryogenic projects. 
disentangle a WIMP signal from neutron backgrounds using different targets ( $\mathrm{Si}$ and $\mathrm{Ge}$ ), for which WIMP and neutron response differ. The observation of both effects would be highly complementary in making the argument for neutralino dark matter a convincing one.

Finally, if the present DAMA annual modulation claim were to survive the test of time, Majorana would not only confirm it as a $>50$-sigma effect, but also reveal a second WIMP signature: the tiny, $\sim 0.1 \%$ daily rate modulation arising from the coupling of the rotational speed of the Earth $(\sim 0.45 \mathrm{~km} / \mathrm{s}$ near the equator) to orbital and solar speeds through the halo[Co199]. Unfortunately, for cross sections any lower than in the DAMA favored region of Fig. 1 an exposure even larger than 5,000 kg-y would be required to detect this.

While its main goal is to measure the effective Majorana mass of the neutrino, the singular characteristics of the Majorana detector make it a promising tool in the quest for dark matter. The projected WIMP sensitivity is competitive even under the conservative background assumptions made. It must be noted that the "signal-to-noise" limits depicted here do not rely on a long exposure, and it is expected to make immediate improvements over the existing Ge detector dark matter limits during Phase 1. Thereafter, the new lowenergy background information and associated Monte Carlo studies will be used to project (and then execute) further incremental improvements in the sensitivity of the experiment.

\section{Other Science: Exploitation of Majorana Data for Axion Searches}

The Majorana experiment will have 500 times the mass, twice the energy range, will run 10 times as long, and should be able to reduce the background over that of SOLAX, a previous germanium-based axion search, by at least a factor of 50. This should translate into a bound on the axion-to-two-photon coupling constant of a few times $10^{-10} / \mathrm{GeV}[\operatorname{Ira} 00]$.

The theoretical motivation and history of experimental searches for axions has been recently reviewed by Rosenberg and van Bibber[Ros01]. Quantum chromodynamics (QCD) is very successful in describing many features of the strong interactions.

However, the complete QCD Lagrangian contains some symmetries that do not survive quantum effects. Classically, complex terms that break these symmetries can be rotated away if the fermion fields have chiral invariant interactions. At the quantum level, however, such transformations involve a phase angle $(\theta)$ that is not arbitrary. Although it must be zero so as not to introduce a T-violating term, the transformation that brings the quark-matrix to a real, diagonal chirally invariant form does not have a zero phase angle $(\theta)$. Since QCD respects CPT symmetry, this phase leads to CP-violation, which predicts an electric dipole moment a factor of $10^{11}$ larger than the experimental upper bound[Pec89].

Peccei and Quinn solved this problem by recognizing that the quark mass-matrix is a function of vacuum expectation values (VEVs) of weakly coupled scalar fields $(\varphi)$. The 
VEVs are determined by minimization of the associated potential V $(\varphi)$. They assumed that the Lagrangian has a global U(1) chiral symmetry under which the determinant of the mass-matrix changes by a phase fixed only by instanton effects that spontaneously break the global U(1) symmetry. This results in an additional phase that cancels the offending one that leads to the large CP-violation[Pec77].

Spontaneous symmetry-breaking processes naturally produce Goldstone-bosons. The Goldstone-boson arising from the breaking of the Peccei-Quinn symmetry is called the axion. In two independent papers Weinberg[Wei78], and Wilczek[Wil78] pointed out that these axions could have physically observable and important properties.

The conventional wisdom says they could possibly couple to electrons, to photons, or directly to hadrons. Accordingly, they might have been produced in the Big Bang, and therefore are candidates for cold dark matter (CDM). They might also be produced in stellar burning and in stellar collapse, etc.

The Peccei-Quinn axion is the most plausible solution to the strong CP problem found to date. This fact continues to motivate experimental searches. The technique presented below is one initiated by members of the Majorana collaboration and is an interesting side application of the Majorana array of detectors.

The first technique aiming at the detection of solar axions was suggested by Sikivie in 1983[Sik83]. It involves Primakoff axion-to-photon conversion in an intense transverse magnetic field, in what is called a magnetic helioscope. This technique is highly efficient for very light mass axions: an experiment under construction at CERN uses a $10 \mathrm{~m}$ long magnet with a transverse magnetic field of 10 Tesla. This experiment (CAST) will reach the maximum sensitivity that the helioscope technique can offer using existing or conceivable magnet technology[Avi01]. The projected sensitivity is better than astrophysical constraints based on the lifetime of red giants[Raf96]. This technique is nevertheless limited to axion masses up to about $0.1 \mathrm{eV}$. This limitation is due to the requirement that axion and photon wave functions stay in phase throughout the magnet (coherence loss)[Zio99]. In order to search for solar axions with masses $>0.1 \mathrm{eV}$ it is necessary to fill the magnet bores with a gas that will act like plasma, effectively slowing the speed of the photon, allowing it to remain coherent with the slower massive axion. This addition to the technique has nevertheless its own limitations[Zio99]: for axion masses larger than $\sim 1 \mathrm{eV}$ the needed gas density would require a pressure of 15 atmospheres and would absorb the axion-induced photons (the signal) before they can reach the detectors. For masses beyond this range one needs a different experimental technique.

To address this problem, several members of the Majorana collaboration, at the time working as members of the SOLAX collaboration, designed a technique using an ultra low background germanium detector: it aims to detect photons coherently converted by Primakoff scattering off the crystalline planes of a germanium detector at times when the line of sight from the detector to the Sun makes an angle with one of the planes that fulfills a Bragg coherence condition. The theory describing the expected conversion rate 
was developed by Creswick et al.[Cre98]. A complete description of how such data are analyzed was published in the proceedings of AXION-98[Avi99]. An experiment was performed in the Hiparsa iron mine in Sierra Grande, Argentina, during which $1.94 \mathrm{~kg}-$ years of data were collected. Each event in the energy region of interest was marked with the exact Julian time. For each day of every year, a pattern of the expected times for Bragg coherence was calculated for use in the analysis of the data. The resulting lower bound on the axion-to-two-photon coupling constant was $2.7 \times 10^{-9} / \mathrm{GeV}$. A complete description of the experiment and of the data analysis was published in Physical Review Letters[Avi98].

The SOLAX experiment effectively served as a demonstration of the principle of detecting axions with single crystals. In SOLAX the crystal axis direction was unknown and the data had to be analyzed for every degree of rotation about the symmetry axis of the detector, which was along the radius of the earth. The Majorana experiment will have 500 times the mass of the SOLAX experiment, with crystal
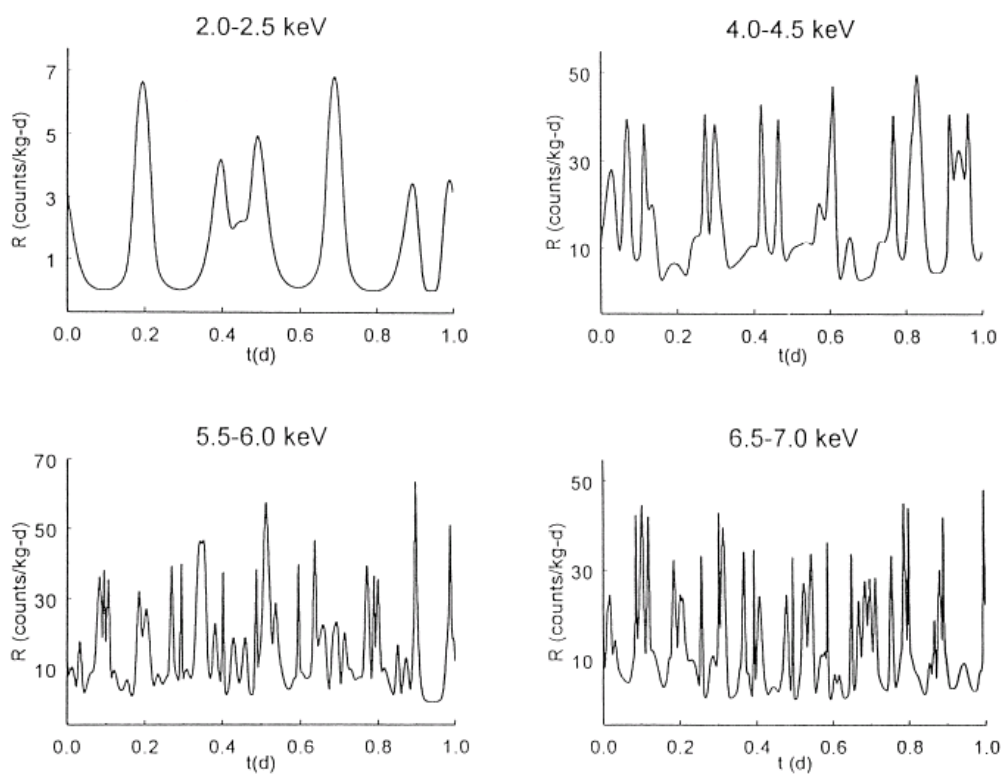

Figure 2-4 Theoretical prediction [5] of the count rate of photons converted $\left(\mathrm{g}_{\alpha \gamma \gamma}=10^{-8} \mathrm{GeV}^{-1}\right)$ from axions incident at a Bragg angle, for a detector at Sierra Grande, Argentina. planes fixed as desired.

In a more granular experiment like Majorana, the axes can be oriented in a variety of ways so that background can be subtracted.

There are two significant improvements that can be made in the quality of the solar axion data obtained with the Majorana experiment, relative to that obtained by SOLAX. First, in SOLAX the low-energy background was high due to microphonic noise and cosmicray neutrons associated with an overburden of less than 1,000 mwe. Secondly, the pulseshape discrimination technique used in the SOLAX experiment was a crude, first generation technique. Recent developments have resulted in very sophisticated digital techniques for pulse-shape discrimination. The digital electronics planned for the Majorana experiment, described elsewhere in this proposal, should allow an energy threshold below $1 \mathrm{keV}$. Compared with the 4-keV threshold of the SOLAX experiment, this implies Majorana will be sensitive to significantly more of the critical low-energy fraction of the signal. 
As summarized above, the Majorana experiment will have much more mass, cover a crucial part of the axion response energy range, gather much more exposure, and have a much lower background than SOLAX. This translates into a bound on the axion-to-twophoton coupling constant of a few times $10^{-10} / \mathrm{GeV}$ [Ira00].

To improve on this expected Majorana bound will be extremely difficult and expensive. Nevertheless, there are other experiments being planned that involve hundreds of kilograms of germanium detectors, bolometers, and scintillators. By careful application of the data analysis technique in [Avi98] it is possible to add the parameters resulting from the maximum likelihood analyses of the different experiments. It can be shown that this procedure is legitimate, and that the combination of experimental results in this way does not depend on the location of the experiment, the crystal structure, or the orientation.

This technique will allow exploration of a significant portion of the axion model space. Its main advantage is that it is sensitive to axion rest masses well beyond $1 \mathrm{keV}$, surpassing any other existing methods in this mass range.

\subsection{Educational Outcomes}

The Majorana project contains elements of several disciplines, and can be expected to produce advanced academic degrees on several fronts. The project opportunities for undergraduate and graduate students in physics, and mechanical, electrical, and computer engineering cover many diverse challenges. The Majorana Collaboration institutions have produced many successful Ph.D. and Master's degree students in science and technology areas closely related to the Majorana project, and are cultivating graduate and undergraduate students now in anticipation of a number of exciting degrees.

We anticipate that students from our several organizations will work at some combination of their home institutions, PNNL (through the Office of Fellowship Programs), and the experiment location during the course of their degree work. In addition, some of our faculty collaborators may also work for extended periods at PNNL through the OFP aegis. OFP allows faculty and students relatively free access to the laboratory and in some cases allows DOE or PNNL support though use of direct or LDRD funding, at low overhead rates.

Several physics Ph.D. or Master's topics can be predicted with certainty:

\section{Master's level topics}

- Digital Filter Models for Optimal Low-Energy Threshold Operation of the Majorana Experiment

- Optimization of HPGe Detector Segmentation for Background Rejection and Process Yield

- Monte-Carlo Analysis of Detector Segment Self-Shielding for the Majorana Experiment

- Suppressing Cosmic Muon Induced Neutrons in an Underground Laboratory Scenario

- Identifying Low-Energy Backgrounds in an Ultra-Low Level Germanium Spectrometer

\section{Doctoral level topics}

- Confirmation/Denial of DAMA Dark Matter Mass Result Based on the Majorana Phase 2 Experiment 
- Annual Modulation Dark Matter Sensitivity of the Majorana Phase 3 Experiment

- New Limits on Existence of Solar Axions from Majorana Phase 2 Data

- Precision Re-measurement of $2 v$ Double-Beta Decay of ${ }^{76} \mathrm{Ge}$ Using Multiplicity Cuts

- Measurement of the $2 v$ Double-Beta Decay to the Excited State of ${ }^{82} \mathrm{Se},{ }^{96} \mathrm{Zr},{ }^{100} \mathrm{Mo},{ }^{130} \mathrm{Te}$, or ${ }^{150} \mathrm{Nd}$

- New Bound on 0v Double-Beta Decay with the Emission of a Majoron

- New Limits on Existence of Solar Axions from Majorana Phase 3 Data

- New Limits/Measurement on Majorana Mass of Electron Neutrino

The Majorana experiment will also provide many opportunities for the educational development of students in a non-traditional or cross-disciplinary way. A significant number of Master's theses and Doctoral dissertations are expected to accompany the collaboration's progress toward and through its final stage. A brief list of some possible degree titles follows:

\section{Master's level topics}

- Mechanical and Thermal Design and Analysis of an Ultra-Low Background Cryostat for the Majorana Phase-3 Experiment (mechanical engineering)

- Signal Routing for the Majorana Project: Ultra-Low Background Transmission Lines with Low Thermal Conductivity (electrical engineering, physics)

- Monte-Carlo simulation of the Majorana Project Phase-3 Integrated Active and Passive Shield (physics)

- A Control System and Data Server for the Majorana Project Phase-3 Installation (physics, computer science)

- Time-Correlation Analysis of Data from the Majorana Project Phase-3 Double-Beta Decay Experiment (physics, mathematics)

- Failure Prediction for the Majorana Project Phase-3 Apparatus (physics, mathematics)

- Optimizing Dark-Matter Sensitivity for the Majorana Project Phase-3 Experiment (physics)

- Shield Mechanical Design and Optimization for the Majorana Project Phase-3 Experiment (mechanical engineering)

- Failure Prediction of Solid State Systems Based on Regular Time Series Data (statistics)

- Alternate Cooling Methods for HPGe Detectors (physics, mechanical engineering)

\section{Doctoral level topics}

- Process Control and Material Quality Monitoring for the Electroforming of Ultra-Low Background Copper (chemistry, chemical engineering, physics)

- Pulse-Shape Discrimination for Background Rejection in the Majorana Project Phase-3 Segmented Detector Array (physics, statistics)

- A High Bandwidth Charge-Integrating Preamplifier Suitable for Ultra-low-background, Cryogenic Sensor Signals (electrical engineering)

- Interaction Localization with HPGe Detector Segmentation and Pulse-Shape Discrimination (physics, electrical engineering) 


\subsection{Implementation of Majorana Project Approach}

The desired outcome of the Majorana experiment is the discovery of the effective Majorana mass of the electron neutrino, and the approach is measuring the rate of zeroneutrino double-beta decay ( $0 v \beta \beta$-decay). However, rather than approaching this project as a single monolithic task, the Collaboration has opted to create a phased approach which separates the project components along the lines of incremental development of new background suppression techniques. Each of these phases comes with distinct physics goals, and will serve to prepare the Collaboration in terms of analysis and acquisition software, specialized copper electroforming, detector manufacturing, and efficient contracting for the enriched material for creating the full set of germanium detectors.

\subsection{Design of Majorana}

\section{Backgrounds}

The origin of the technology for the measurement of ${ }^{76} \mathrm{Ge}$ double-beta decay goes back to the clever introduction of the internal source technique by Fiorini[Fio67]. This method allows the experimenter to use a high-resolution germanium $\gamma$-ray spectrometer to measure the radiation that is emitted from the germanium itself. Initially, natural germanium spectrometers were simply shielded from environmental backgrounds to achieve the first limits. The limitation of this technique for application to ${ }^{76} \mathrm{Ge}$ double-beta decay is the quantity of background signals observed at the desired detection energy, $2039 \mathrm{keV}$. While the $0 v \beta \beta$-decay energy is above most ubiquitous environmental radiation, it is does not exclude all backgrounds. However, over time, sources of radioactivity have been identified and removed resulting in greatly improved sensitivity to longer half-lives.

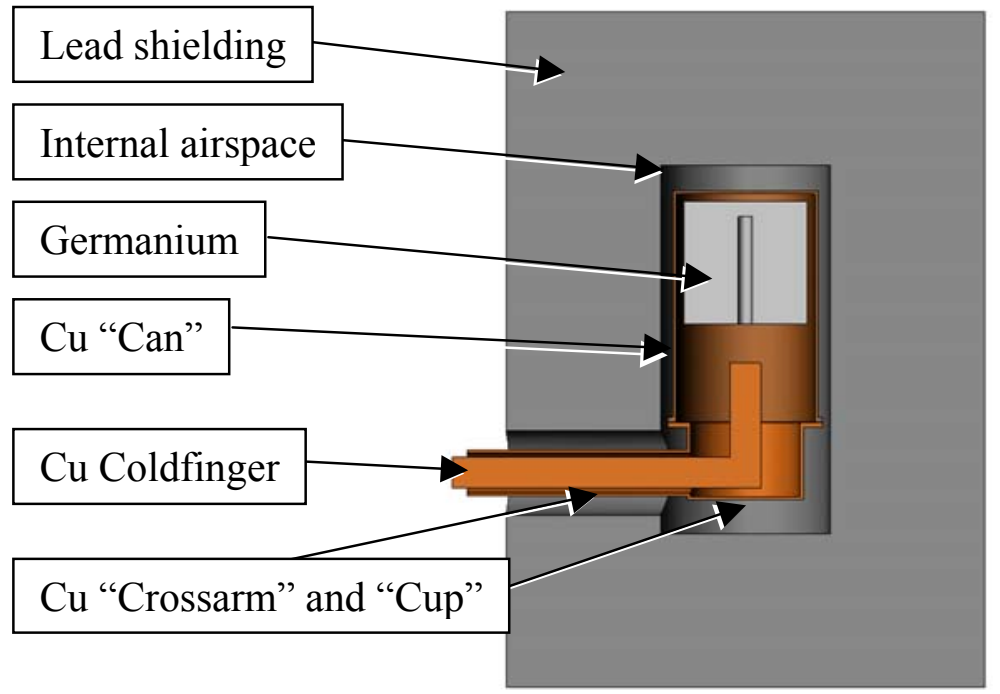

Figure 3-1 Typical germanium detector arrangement. The volume of $\mathrm{Ge}$ is about $400 \mathrm{cc}(2.1 \mathrm{~kg})$ and the volume of $\mathrm{Cu}$ is Can: 97 cc (0.8 kg); Crossarm + Cup: 63 cc (0.6 kg) + Coldfinger: $82 \mathrm{cc}(0.7 \mathrm{~kg})$; Total: $242 \mathrm{cc}(2.1 \mathrm{~kg})$.

We begin our discussion with a schematic setup, shown in figure 3-1, in which the generic arrangement of germanium, structural materials (largely copper) and lead shielding are shown. The detector may be configured at the time of production either as p-type or n-type, depending on the majority impurities in the material. P-type detectors have an outer $0.5-0.75 \mathrm{~mm}$ thick "dead" layer of highly-doped P-type germanium that is 
insensitive to radiation. An n-type detector also has this dead layer, but on the surface of the inner hole which provides the electrical contact and therefore provides little shielding against external radiation. Dead layers have the effect of screening the detector from lowenergy radiations and alpha particles from external or surface-layer activities. Thus, lowenergy backgrounds in the detector are either within the inner materials or on the innermost surfaces. Higher energy backgrounds, however, are usually penetrating $\gamma$ rays originating from the germanium, copper, the inner region of lead, or cosmic ray muons and muon induced neutrons and gamma rays penetrating the bulk of the lead. Since in this arrangement the germanium is surrounded by copper, any radiations emanating from the lead must pass through a small amount of copper to reach the germanium.

Since the earliest use of germanium in the internal source configuration, several improvements to this schematic design have been added. In approximate order these include:

$>$ Cosmic veto shielding

$>$ Deep underground locations

$>$ Detector construction using screened low-background materials (copper)

$>$ Detector construction using electroformed copper

$>$ Ultra-pure shielding materials

$>$ Low-cosmic-ray exposure germanium and copper

$>$ Germanium enriched from $7.8 \%$ to $85 \%$ in ${ }^{76} \mathrm{Ge}$

$>$ Signal processing

$>$ Coming soon: Detector segmentation

The point of this recapitulation (see figure 3-2) is that a $\sim 20$-year R\&D program by Majorana collaboration members has preceded this proposal as we struggled to understand each successive background. For instance, the same germanium crystal was used for the upper curves in figure 3-2. On the occasions when this detector was rebuilt and returned underground, the effects of cosmogenic activation were observed and quantified. Similarly, the effort to produce ultra-pure support structures has resulted in materials rivaling the germanium itself

in radiopurity[Bro95].

\section{Cosmogenics}

Based on observation of increased activity in well-known detectors that were brought to the surface for service, the limiting background was found to be the activity in the germanium itself[Avi92a], cosmogenic ${ }^{68} \mathrm{Ge}$ and ${ }^{60} \mathrm{Co}$, in particular.
Table 3-1. Detectable spallation isotopes in germanium. All have electron capture decay modes (or EC daughters).

\begin{tabular}{ccc}
\hline Isotope & $\mathrm{T}_{1 / 2}$ & $\gamma / \mathrm{x}$ Energy \\
\hline${ }^{54} \mathrm{Mn}$ & $312 \mathrm{~d}$ & $834.8 \mathrm{keV}$ \\
${ }^{57} \mathrm{Co}$ & $272 \mathrm{~d}$ & $122.1 \mathrm{keV}$ \\
${ }^{58} \mathrm{Co}$ & $70.9 \mathrm{~d}$ & $810.8 \mathrm{keV}$ \\
${ }^{65} \mathrm{Zn}$ & $244 \mathrm{~d}$ & $1115.52 \mathrm{keV}$ \\
${ }^{68} \mathrm{Ge}$ & $271 \mathrm{~d}$ & $10.4 \mathrm{keV}$ \\
\hline
\end{tabular}




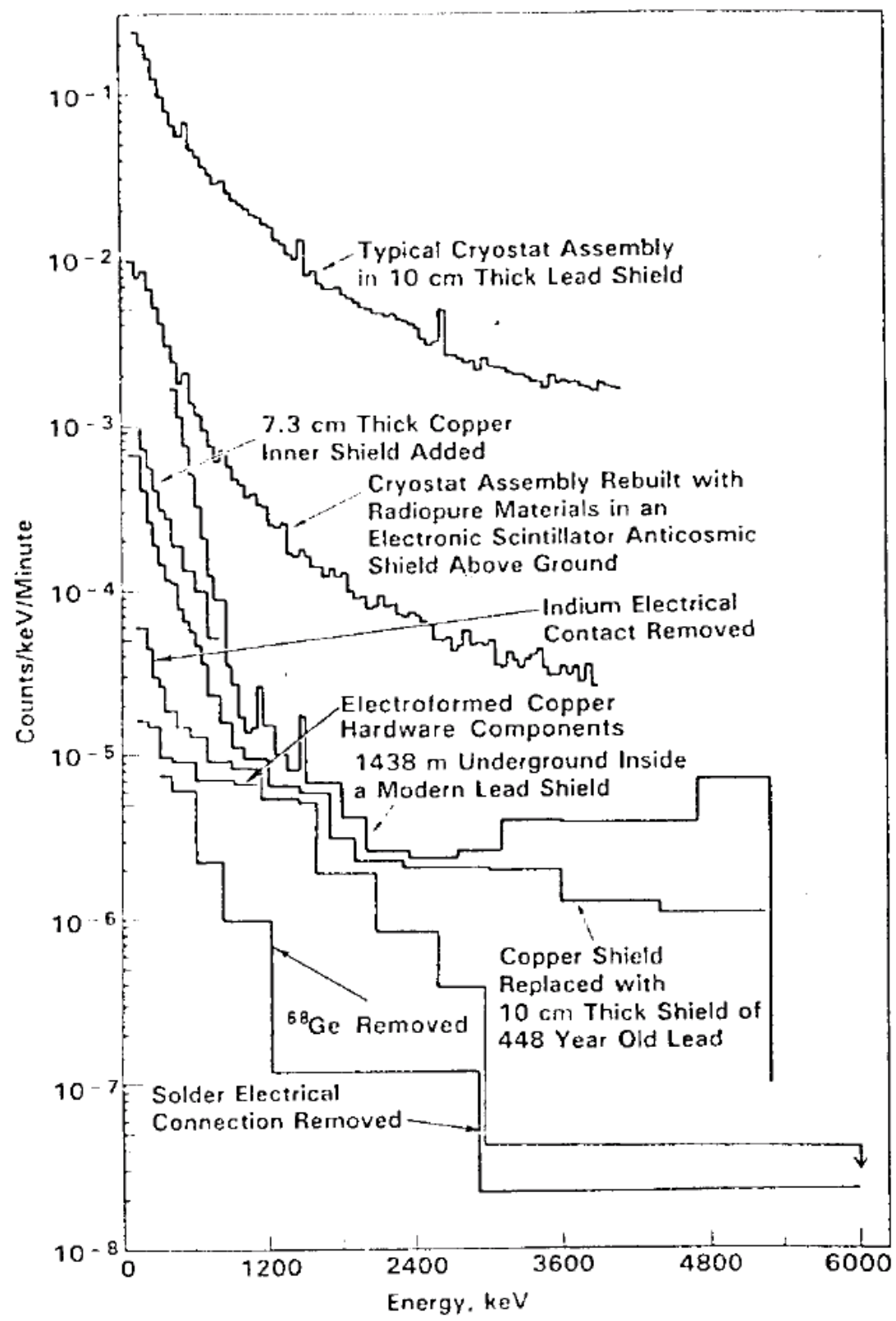

Figure 3-2 A summary of spectral improvement from about 1980 to 1990. Spectra include aboveground and below ground systems[Bro90].

Many gamma-emitting isotopes are difficult to detect when inside a detector. For instance, ${ }^{60} \mathrm{Co}$ decays with the emission of a beta particle followed by two gamma rays. Because these are summed together, the beta continuum smears out the gamma-ray peaks. Isotopes from the neutron deficient side of the nuclide chart undergo electron capture (EC) as well as positron decay. When no positron is emitted, the gamma-ray peaks may be found, except that the subsequent atomic de-excitation of the daughter atom must sum in the germanium as well. In the case of ${ }^{68} \mathrm{Ge}$, there is a difficult-todistinguish positron decay followed by the $\mathrm{EC}$ decay of ${ }^{68} \mathrm{Ga}\left(\mathrm{T}_{1 / 2}=68.1 \mathrm{~m}\right)$. The electron capture is most often of a $\mathrm{k}$-shell electron, and a cascade of $\mathrm{x}$-rays follow, which 
sum to the binding energy of the k-shell electron (Note: the $\mathrm{k} / \mathrm{l} / \mathrm{m}$ capture ratios must be used to correct the integral of the $10.367 \mathrm{keV}$ peak when calculating the number of ${ }^{68} \mathrm{Ga} /{ }^{68} \mathrm{Ge}$ decays). The observation of this summed energy deposition has been critical in the past to validate the ${ }^{68} \mathrm{Ge}$ production rate and to allow observationally-based spectral stripping of the ${ }^{68} \mathrm{Ge}$ spectral shape from IGEX data, a key confidence-building feature. This effect shows up as a shift of the expected $\gamma$ ray by the electron binding energy. For instance, the familiar ${ }^{65} \mathrm{Zn}$ line

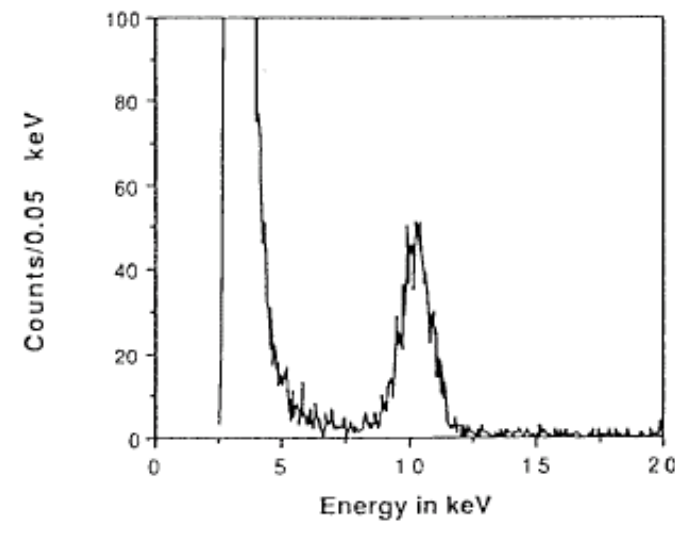

Figure 3-3 Observation of 10.367 $\mathrm{keV}$ binding energy from ${ }^{68} \mathrm{Ga}$ EC[Bro90]. at $1115.52 \mathrm{keV}$ would be found at $1115.52+9.659=1125.18 \mathrm{keV}$. In fact, this line led our group to the realization of the importance of cosmogenics inside the germanium

The need to predict the concentration of cosmogenics led to the computation of spallation cross sections. This was accomplished using ISABEL[Yar81], a code for computing compound nuclear reactions. Many successive simulations at a range of neutron projectile energies from 10 to $2000 \mathrm{MeV}$ allowed the computation of the reaction probability. These probabilities were then convolved with neutron spectra (Hess[Hes59], $\mathrm{L} \& \mathrm{P}[\mathrm{Lal67]})$ to obtain a predicted rate of production while above ground. To validate this computation, an experiment was carried out.

Before our collaboration began assembling and servicing germanium detectors underground, we were obliged to return the detectors to a manufacturer for maintenance. One such repair required that a detector long underground ( $>950$ days) be exposed to the surface cosmic-ray flux for about 150 d. During 250 -d

Table 3-2. Theoretical and experimental spallation rates in atoms per day per $\mathrm{kg}$ at sea level.

\begin{tabular}{cccccc}
\hline Isotope & \multicolumn{3}{c}{ Natural Ge } & \multicolumn{2}{c}{ Enriched Ge } \\
\hline & L\&P & Hess & Experiment & L\&P & Hess \\
${ }^{54} \mathrm{Mn}$ & 0.93 & 2.7 & $3.3 \pm 0.8$ & 0.37 & 1.4 \\
${ }^{57} \mathrm{Co}$ & 1.7 & 4.4 & $2.9 \pm 0.4$ & 0.28 & 1.0 \\
${ }^{58} \mathrm{Co}$ & 2.30 & 5.3 & $3.5 \pm 0.9$ & 0.59 & 1.8 \\
${ }^{65} \mathrm{Zn}$ & 24.6 & 34.4 & $38 \pm 6$ & 3.12 & 6.4 \\
${ }^{68} \mathrm{Ge}$ & 22.9 & 29.6 & $30 \pm 7$ & 0.54 & 0.94 \\
\hline
\end{tabular}

period following the return underground, the production rates of the isotopes in Table 3-1 were experimentally determined. A comparison is shown in Table 3-2.

Predicted Germanium Cosmogenic Backgrounds at 2039 keV

Given that we have established that the cosmogenic radionuclides in natural germanium may be calculated with an uncertainty of $30 \%$ to $200 \%$, we can calculate the production of cosmogenics in enriched material as a guide to what we expect in the full Majorana experiment. Observing in Table 3-2 that the convolution of the Hess neutron spectrum with the isotope-weighted, computed cross-sections produces reasonable agreement, we 
can use the Hess convolution with the enriched isotopic weighting to estimate the production rates for enriched material while on the surface. These rates are one or two atoms per day per $\mathrm{kg}$, except for ${ }^{65} \mathrm{Zn}$. The computed contribution of each activation isotope to the $2039 \mathrm{keV}$ region of interest is taken in to consideration in the sensitivity calculation of Section 2.3.

\section{Contribution to 2039-keV Background from Copper Support Materials}

The copper that forms the vacuum jacket, cold finger, and other structural or shielding materials can contribute either through included natural contamination (e.g. ${ }^{238} \mathrm{U}$ and ${ }^{232}$ Th series) or through cosmogenic activity. Copper may contain many of the same activation products as germanium, and may have a higher spallation cross-section since the $\Delta \mathrm{Z}$ from $\mathrm{Cu}$ to ${ }^{54} \mathrm{Mn}$ and ${ }^{57,58,60} \mathrm{Co}$ is less than from $\mathrm{Ge}$.

The only danger to the $2039 \mathrm{keV}$ region from activation in copper is the sum of both ${ }^{60} \mathrm{Co}$ gamma rays, which is quite unlikely even for the layout described in figure 3-1. The efficiency for this is $0.6 \%$ in a crystal $<1 \mathrm{~cm}$ from the copper, and far less for germanium farther from the source, say from an adjacent detector vacuum jacket within the same bulk lead shield. However, the activation of these cosmogenic isotopes has been historically suppressed in IGEX detectors by underground storage of copper parts prior to final assembly. For the Majorana experiment, these isotopes may be eliminated completely by electroforming the copper underground from copper anode stock stored underground. Thus the concern should focus on included natural radioactivity.

Uranium and thorium chains can be introduced into the copper by being present in the electroforming bath. A particular element may have more or less affinity for the plating process, depending on its electronegativity. Once an element of the chain is introduced, the equilibrium of that constituent and its daughters begins to be established. As a general rule, after 5 half-lives of the longest chain member, the series will be in secular equilibrium. In a measurement to assay the contribution of the $U$ and $T h$ chains in the copper, daughters of radon, from radon leaking into the internal air space of the detector assembly, will likely be confused with daughters of the $U$ and Th decay chains. For this reason, solid precursors of radon are used to establish the level of solids in the copper.

To create a sensitive assay of the copper, an electroforming campaign was undertaken to produce an inner shield of copper[Bro95] placed between the bulk lead shield and the vacuum jacket. The completed part was of about $9 \mathrm{~kg}$ in mass. A lengthy measurement showed that for the $\mathrm{U}$ series activity was $<25 \mu \mathrm{Bq} / \mathrm{kg}$ of copper and for the Th series about $9 \mu \mathrm{Bq} / \mathrm{kg}$ copper. The only natural isotopes in the copper of concern are ${ }^{214} \mathrm{Bi}$ and ${ }^{208} \mathrm{Tl}$. Although these were not a measurable part of the background measured in an 84day spectrum obtained in early IGEX data at 4000 mwe, these and all other natural isotopes will be suppressed even further by repeated bath recrystalization. This bath recrystalization process has been successful in reducing gross amounts of natural isotopes in the bath, and can be simply extended. 
Traditional Cryostat Design vs. Alternate Cooling

The design of the baseline plan is dependent on our collaboration understanding of the origin of the signals at $2039 \mathrm{keV}$, as explained above. Knowledge of the basic background sources, when combined with the equation for the $T_{1 / 2}$ of the decay mode, explain our development.

Considering only proportionalities (i.e. neglecting units and constants), the generic functional forms of $\mathrm{T}_{1 / 2}$ and $<\mathrm{m}_{\mathrm{e}}>$ are given by:

$$
\begin{aligned}
& T_{1 / 2}=\frac{M T}{C} \\
& \left\langle m_{e}\right\rangle=\frac{1}{\sqrt{T_{1 / 2}}}
\end{aligned}
$$

where $\mathrm{M}$ is the mass of ${ }^{76} \mathrm{Ge}, \mathrm{T}$ is the effective counting time, and $\mathrm{C}$ is the sum of counts attributable to the decay of interest. If backgrounds dominate such that no signals are seen at $2039 \mathrm{keV}$, The $\mathrm{T}_{1 / 2}$ limit would be:

$$
T_{1 / 2}>\frac{M T}{\sqrt{B}}
$$

where $\mathrm{B}$ is the number of background counts in the region of interest. If the dominant backgrounds are in the germanium, as we have shown for previous experiments, the number of background counts is proportional to the product of $\mathrm{M}$ and $\mathrm{T}$ and the functional form of the $\mathrm{T}_{1 / 2}$ limit simplifies to:

$$
T_{1 / 2}>\sqrt{M T}
$$

So doubling the mass of the experiment increases the $\mathrm{T}_{1 / 2}$ by only $40 \%$, and decreases the effective neutrino mass limit by $20 \%$. Doubling the mass of the experiment from $500 \mathrm{~kg}$ to $1000 \mathrm{~kg}$ could cost $\$ 25 \mathrm{M}$ for little return in this scenario.

On the other hand, if one assumes there is no background in the germanium, but there is a limiting and constant background rate (b) in the support structures, then the background would be $\mathrm{B}=\mathrm{bT}$, and the $\mathrm{T}_{1 / 2}$ limit would simplify to:

$$
T_{1 / 2}>M \sqrt{\frac{T}{b}}
$$

And it would behoove the planner to construct an experiment with high mass and exceedingly small amounts of structural materials. This is the focus of most alternate cooling techniques.

We have demonstrated that small amounts of cosmogenic materials will be in the detector crystals, even if manufactured underground. Furthermore we have shown that support 
structures can be readily made without limiting background contributions. Therefore we conclude that the experiment should be designed with a moderate Ge mass and a long (inexpensive) run time. In addition, special emphasis should be placed on electronic suppression methods for backgrounds and in construction techniques that promote very long-lived detectors.

In conclusion, since it has been shown that cosmogenic isotopes have played the most important role in previous germanium experiments and support structures have not, the baseline plan of the Majorana experiment is to plan for and minimize cosmogenic background sources, then concentrate on the next most serious background source, cosmic secondary neutrons. In the event that an alternative cooling/shielding arrangement is found which does not compromise the gains in cosmogenic rejection or neutron suppression, it will be entirely possible to adopt these methods without causing harm to the already-manufactured crystals.

\subsection{Concept for Phased Majorana Approach}

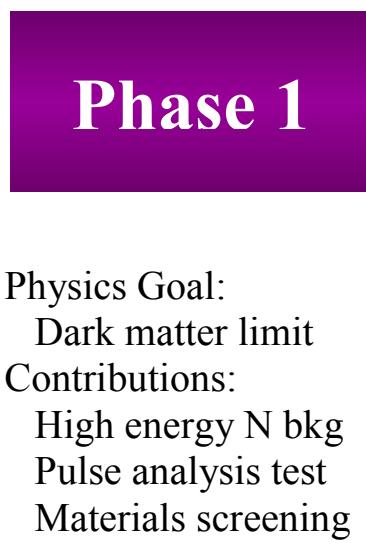

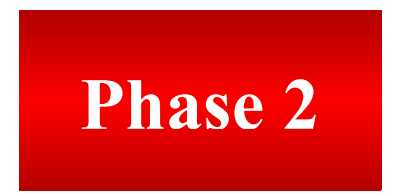
Physics Goal:
Excited State $2 v \beta \beta$
Contributions:
Segmentation test
Materials test
Cryo-design test
Geometry test

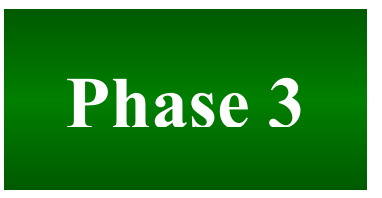

Physics Goal:

Measure neutrino mass

Dirac vs. Majorana

High DM sensitivity

Figure 3-4 Phased approach showing $R \& D$ phases (1 and 2) leading to the ultimate neutrino measurement, Phase 3.

\subsubsection{Phase 1 Description; Science and R\&D Goals}

Phase 1 of the Majorana experiment will be to prepare 4 detectors of varying segmentation designs from more than one manufacturer. We describe segmentation in terms of $\mathrm{k} \times \mathrm{j}$ segments where there are $\mathrm{k}$ segments along the $\mathrm{Z}$ axis of the right circular cylinder and $\mathrm{j}$ segments about the $\phi$ direction. These detectors will vary from $2 \times 4$ segments to $3 \times 6$ segments. Also, the approach for segmentation currently in use 
mandates that the germanium detector must be n-type due to the ease of lithography of the boron implanted contacts on the outside of the crystal. Other options for achieving high detector granularity exist, small (200 g) planar detectors, for example, and are under review.

The main R\&D goal of Phase 1 is the determination of the most effective combination of signal processing and segmentation. Our signal processing and segmentation effort is aimed at separating single-site energy deposition events, such as double-beta decay, from multi-site events, such as ${ }^{68} \mathrm{Ge}$ and ${ }^{60} \mathrm{Co}$ decays. For a single-site energy deposition in a segmented detector, the only segment with a net charge is that containing the deposition site. Furthermore, by comparing induced signals in neighboring segments, one can determine if the signal was actually a multiple site event within a single segment[Vet00].
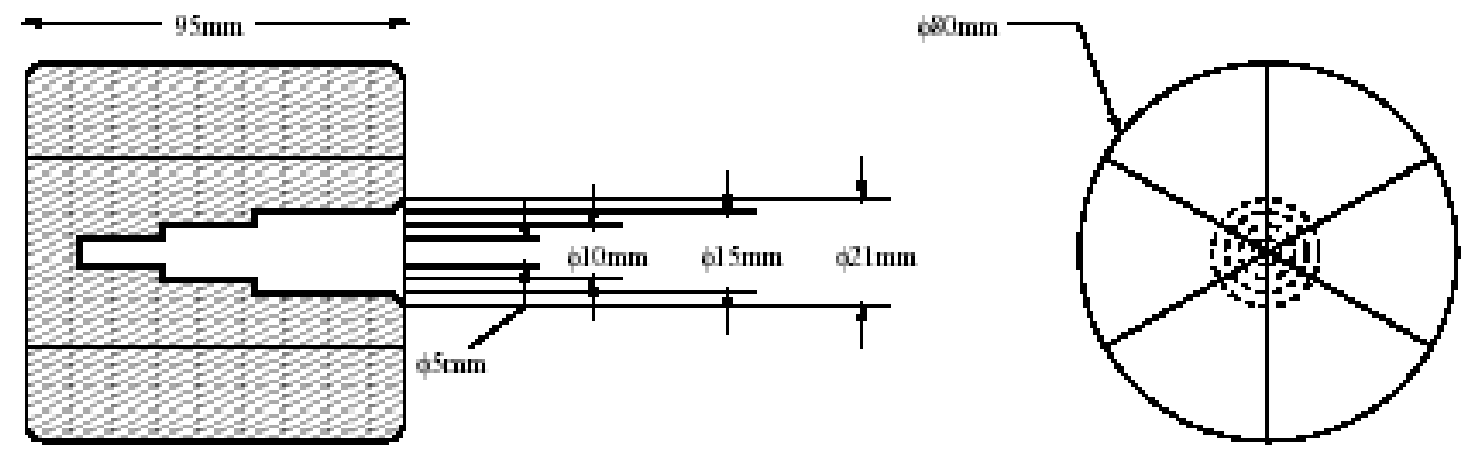

Figure 3-5 Example segmentation of Phase 1 detectors.

Zone refining and crystal pulling both tend to exclude cobalt and other impurities from the solid or crystalline form of germanium. Thus, on the day of crystal creation, there is essentially no ${ }^{60}$ Co present. However, on the surface about 1 atom per kg is created per day. Therefore underground detector manufacturing would be very valuable for suppressing or eliminating one of our chief background sources, ${ }^{60} \mathrm{Co}$. The Phase 1 effort may establish a new method of constructing segmented detectors such that essentially all manufactured crystals would be immediately useful. If successful, this would encourage manufacturers to consider the exciting possibility of manufacturing detectors underground.

\subsubsection{Phase 2 Description; Science and $R \& D$ goals}

Phase 2 of the Majorana experiment will consist of a pair of Phase-1 segmented germanium detectors surrounded by a toroid of 16 fairly large ( $70 \%$ relative efficiency) p-type germanium detectors. The toroidal apparatus will approximate the cryogenic and electronic challenges in the construction of the 21-crystal modules of Phase 3. 


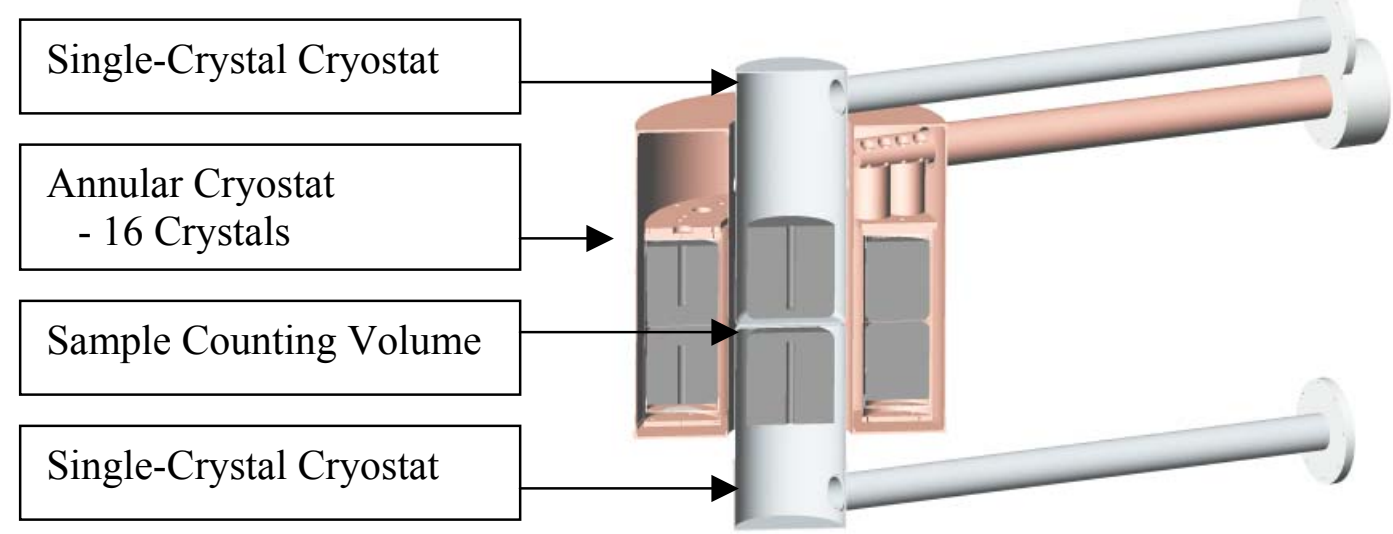

Figure 3-6 Cutaway view of the Phase 2 apparatus with 3 separate cryostats. The inner detectors may be removed and a sample introduced between them. The outer 16 crystals are cooled together much like the 21-crystal Phase 3 apparatus. All the support materials are of electroformed copper.

The first challenge will be the cooling of the detectors as the crystals must operate below about $125 \mathrm{~K}$, optimally around $90 \mathrm{~K}$. In comparison, the noise level for the preamp frontend located near the crystal (for low capacitance and high bandwidth) typically reaches a minimum at $145 \mathrm{~K}$. These temperatures can be simultaneously optimized by engineering the thermal conductivity of the FET mount and clever use of the few milliwatts of power dissipated by each front-end FET.

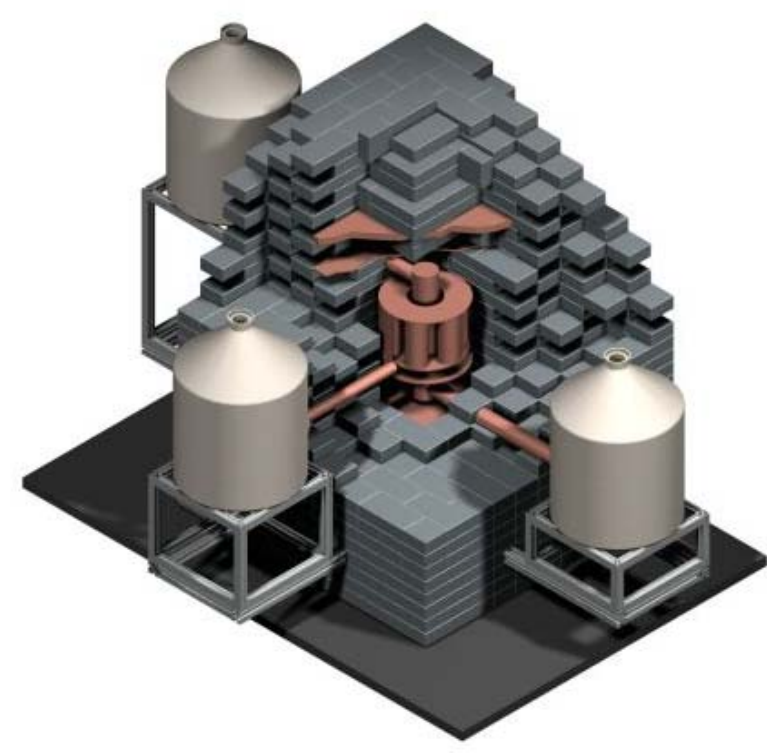

Figure 3-7. Cutaway view of preliminary design of Phase 2 apparatus including shielding.
Another seemingly pedestrian but fundamental challenge is the provision for electronic feedthroughs. Typical germanium detectors have the luxury of a single feedthrough with four contacts for a single detector segment. With as many as 18 segments per crystal, the situation in the Majorana Phase 3 is not as simple. We propose to try new preamp schemes with many of the electrical contacts common. We are considering the use of Multi-Chip Module (MCM) technology that would place the entire pre-amp within the cryostat. While this invites some risk of radiological contamination, the masses are small and modest shielding together with material assay should eliminate this concern. 
The shielding configuration of the Phase 2 apparatus provides a challenge similar in kind but smaller in magnitude than Phase 3. Because the toroidal detector ring will occupy an area in the shield of about $30 \mathrm{~cm}$ by $30 \mathrm{~cm}$, clean support for the lead above will have to be arranged. The current plan is to use structural electroformed copper parts to support a few layers of clean lead, then commercially-obtained OFHC copper sheet to support the rest.

The Monte Carlo simulation of this multi-crystal array poses an interesting task, similar to the analysis of the multi-element data. The effect of various contaminants within multiple materials and locations must be known to guide Phase 3 construction. However it is possible to validate Monte Carlo code by the measurement of signals from known sources in the Phase 2 geometry. Shortly after introduction underground, the detection of photons escaping the inner detectors, for instance from ${ }^{58} \mathrm{Co}\left(\mathrm{T}_{1 / 2}=71 \mathrm{~d}\right)$, will allow testing of suppression of multi-gamma isotope backgrounds using multiple crystals.

Operated either with natural or enriched detectors in the central positions, the Phase 2 apparatus will have several interesting physics applications. First, if fitted with enriched detectors, ${ }^{76} \mathrm{Ge}$ two-neutrino decays to the first $0^{+}$excited state, from which $563 \mathrm{keV}$ and $559 \mathrm{keV} \gamma$ rays are emitted, may be observable. Multiple $\gamma$ rays can be detected from samples, enriched or natural, that are introduced as disks to the space between the two inner detectors resulting in good coincidence efficiencies. Possible isotopes for such sample counting include ${ }^{76} \mathrm{Ge},{ }^{82} \mathrm{Se},{ }^{96} \mathrm{Zr},{ }^{100} \mathrm{Mo},{ }^{130} \mathrm{Te}$, and ${ }^{150} \mathrm{Nd}$. Both the speciallyinstrumented detectors and the high total efficiency (10 times greater than the previous experiments by Majorana Collaboration members) of the toroid allow significant improvements to this type of measurement.

\subsubsection{Phase 3 Description}

Phase 3 of the Majorana experiment is a complete next-generation double-beta decay experiment, using a large quantity of enriched materials, state-of-the-art detector fabrication, deep underground facilities, cutting-edge instrumentation and data analyses, and acquisition of complete state-of-health data. Construction and operation will be done in extremely clean facilities, and all materials will undergo extensive radiological and mass-spectrometry screening.

The apparatus will consist of modules constructed from electroformed copper, each containing many germanium crystals. The baseline plan is to house 21 crystals per cryostat, arranging cryostats in pairs such that 210 crystals of about $2.3 \mathrm{~kg}$ each would comprise the $500 \mathrm{~kg}$ of germanium in the total experiment. If only smaller crystals are possible, the design could easily be extended to 12 cryostats housing a total of 252 crystals of about $2 \mathrm{~kg}$ each. 


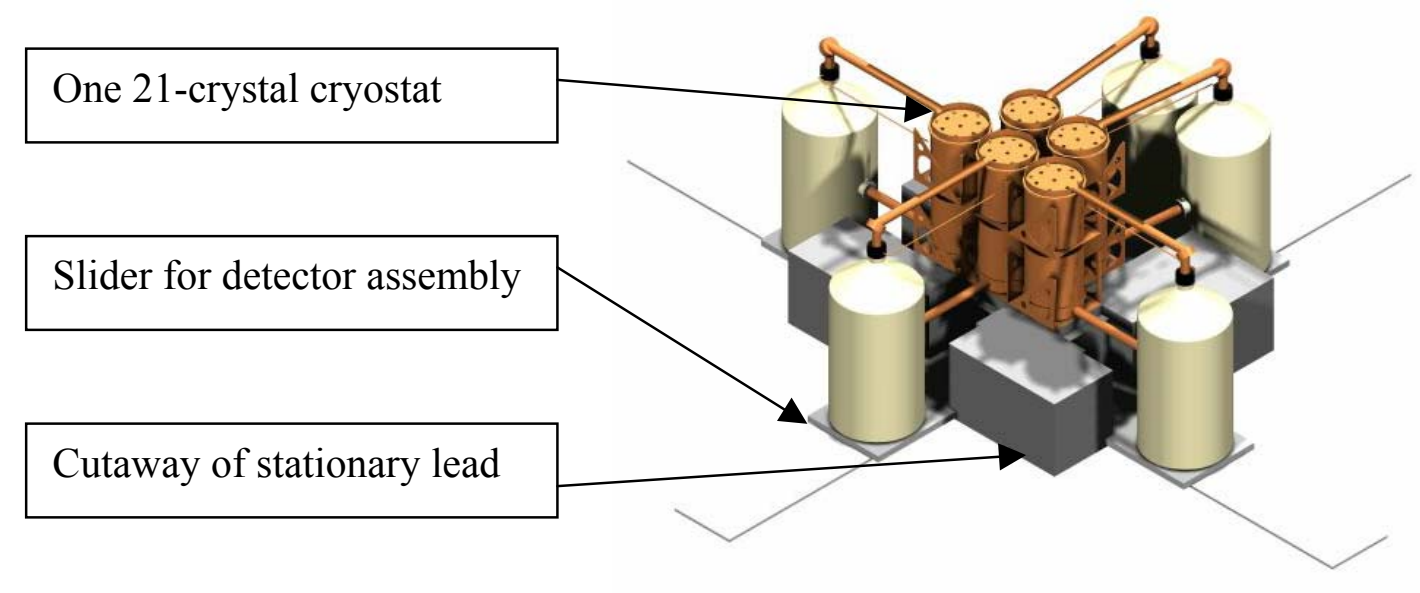

Figure 3-8. Phase 3 apparatus.

This modularity allows the gradual commissioning of the apparatus such that early results could greatly influence the ongoing manufacturing process. Once the crystals are produced and underground, it is possible to avoid the introduction of internal contaminants, so repeated repackaging to take advantage of emerging cryogenic techniques is possible. This gives the opportunity for any background problems at low energy to be mitigated to improve dark matter searches. Modularity also facilitates maintenance; individual modules can be taken off-line to allow repair or upgrades with minimal down-time for the system.

The gradual production of detectors also allows stock materials to be located underground for years in advance, meaning that the short-lived cosmogenics seen in germanium and copper will eventually become undetectable even at detector commissioning.

\subsection{Isotopic Material Separation}

The major requirement of the Majorana project is the procurement of the enriched germanium. Previous double-beta decay experiments have simply borrowed the enriched material from Russian collaborators. Due to wastage in the processing and manufacturing, certain complications can arise in the eventual return of the material. The scale of the next generation of double-beta decay experiments, both in mass and duration preclude the possibility of borrowing millions of dollars worth of material.

The Majorana Collaboration has begun investigating the aspects of enrichment both as relating to science and project management. ITEP has played a crucial role in the investigations, largely because of their previous involvement in enrichment activities. Several discussions with the Electrochemical Plant (ECP) of Zelenogorsk have elucidated the main challenges in the enrichment process, which are simply financial and not technical. In other words, the Collaboration has been assured that the ECP can produce 
germanium of the same purity and enrichment as has been provided for several doublebeta decay experiments in the past, including IGEX and Heidelberg-Moscow.

\subsubsection{Enrichment Costs and Schedule}

The ECP presently has capacity to produce $30 \mathrm{~kg} / \mathrm{y}$ of material to the previous state standards used for double beta decay. Provided an investment is made in increased production capacity, matched by ECP, of $\$ 50 \mathrm{k}$, this capacity can be increased to $50 \mathrm{~kg} / \mathrm{y}$. They have recently quoted the Majorana collaboration that prices for germanium produced under the 30 or $50 \mathrm{~kg} / \mathrm{y}$ rates will be $\$ 56 \mathrm{k} / \mathrm{kg}$. (See Appendix 1: Enrichment)

\subsubsection{Production of $100 \mathrm{~kg} / \mathrm{yr}$}

ECP has offered that for an estimated $\$ 3.5 \mathrm{M}$ the production capacity could be increased to $100 \mathrm{~kg} / \mathrm{y}$. Given that the first year or two would be at 'low' production rates $(20-50$ $\mathrm{kg} / \mathrm{y}$ ), a total production period of seven years would result. This is our baseline plan, on which we base sensitivity estimates. This is quite a long commissioning period, given that the experiment running time would be an additional ten years. ECP estimates that production under these conditions would cost about $\$ 45 \mathrm{k} / \mathrm{kg}$.

\subsubsection{Production of $200 \mathrm{~kg} / \mathrm{yr}$}

The ECP has offered that for an investment of $\$ 5 \mathrm{M}$ in production capacity improvements, an annual production of $200 \mathrm{~kg}$ could be achieved. This production rate means that only two years of main production would be required in addition to the startup period. A total of four to five years of production would substantially speed the commissioning of the Phase 3 apparatus. ECP estimates that production under these conditions would cost about $\$ 45 \mathrm{k} / \mathrm{kg}$.

\subsubsection{Other production facilities}

So far the Majorana Collaboration has investigated only one producer of enriched materials. It may be possible, by contracting with more than one enrichment facility to produce germanium at a net higher rate.

\subsubsection{Schedule risk}

Due to the changing condition of international relations, there is no long-term guarantee that the products of the ECP will continue to be available, or that the Russian government will continue to subsidize the operation of ECP.

In addition, Russia is currently experiencing about $15 \%$ dollar inflation. We calculate that even with only $10 \%$ dollar inflation, the faster $200 \mathrm{~kg} / \mathrm{y}$ production would save $\$ 1.5 \mathrm{M}$ over the slower $100 \mathrm{~kg} / \mathrm{y}$ rate, even with the production improvement investment taken into consideration.

For these project management reasons, as well as the urgent nature of the interest in the properties of the neutrino, the Majorana Collaboration recommends the highest available rate as the best option. 


\subsubsection{Contracting Options}

From the project management perspective, there are several reasonable possibilities for the financial management of the enrichment activity. These involve procurement and contracting out of several US institutions, with various positive and negative features for each

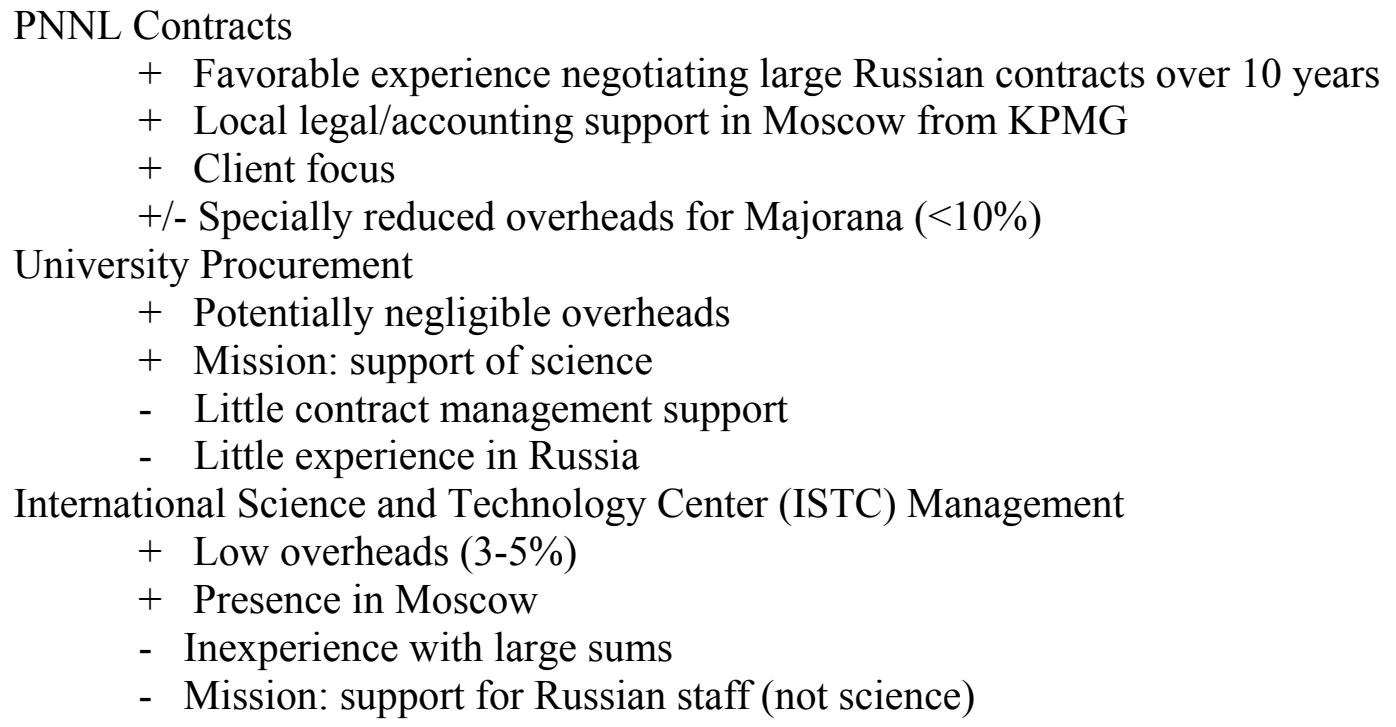

Our intended approach is to try PNNL contracting to determine if an attractive reduction in cost is possible, weighed against the overheads of national lab contracting fees. If this is not satisfactory, university contracting is our contracting fallback. If the source of the funding makes it programmatically necessary to use ISTC (e.g. in the event of State funding of enrichment), we will need to work to assure that the ISTC management is sufficiently focused on our project success. ISTC is not the favored approach by the Russian suppliers.

\subsubsection{Shipping}

While the basic quote from ECP includes shipping via air from ECP to a point of entry into the US via DHL or other carrier, it may not be in the scientific best interest for the material to ship in this way. The high energy neutrons which cause about one atom per day each of the main cosmogenics $\left({ }^{68} \mathrm{Ge}\right.$ and $\left.{ }^{60} \mathrm{Co}\right)$ are produced in cosmic-ray reactions in the atmosphere. This high-energy neutron flux is about 100 times more intense at the cruising altitude of aircraft. Thus, 12 hours at 12,000 meters would be the equivalent of 50 days exposure. While land/sea transit would be much slower and involve some complicated border crossings, the neutron exposure would be half or less, and could be much less if clever shielding and placement were pursued.

Because a 3 month shipment at $200 \mathrm{~kg} / \mathrm{y}$ would be $50 \mathrm{~kg}$ of metal (actually more of oxide), the shipments will be escorted. Assuming an oxide density of about $2.0 \mathrm{~g} / \mathrm{cc}$, the volume of a quarterly shipment would be a cube of about $30 \mathrm{~cm}$, or one cubic foot. Some shielding of this amount of material should be possible. If a factor of two to three in 
neutron exposure reduction could be accomplished, the air transport would be competitive with land/sea with fewer complications of customs and border crossings.

\subsubsection{Taxes, Duties, Customs}

While the ECP quote theoretically includes Russian taxes, duties and customs, we wish to explore this on a relatively small shipment. We propose to involve KPMG Moscow to estimate the potential for difficulty in this area. Further, the Collaboration will explore the tax exemption of isotopes generally in the US.

\subsection{Majorana Phase 3 Implementation}

The 500-kg, ten-year, near-zero-background Majorana experiment depends on the completion of many tasks before the first byte of Phase 3 data may be acquired. These tasks include extensive modeling of the crystals, their environment, and the relevant known physics, to optimize the entire design and to refine sensitivity estimates. Germanium will be enriched and crystals grown, purified, and made into detectors. Cryostats, electronics, and shielding are to be designed, constructed, and made operable. The custom data acquisition system required for handling detector segmentation and pulse digitization will be designed, fabricated, and tested. Data analysis techniques will be developed, and we propose to create a collaboratory infrastructure and social structure to maximize the value extracted from Majorana data.

\subsubsection{Monte Carlo Studies}

Monte Carlo studies of the Majorana Phase 3 apparatus will lead the final stages of design and are essential in interpreting the results of the measurement data. The very limited palette and known list of historically observed isotopes serve to simplify the simulation problem. The great majority of the materials used in the experiment include $\mathrm{Pb}, \mathrm{Ge}$, and $\mathrm{Cu}$. On the other hand, it would appear that the complexity of the simulated space (2500 segments in 210 crystals grouped in 21-crystal modules) could create difficulty. Early simulations run by Collaboration members have shown that this complexity can be mastered easily, and full-up simulations have begun.

The goals of the simulation effort can be stated as follows:

What is the response of individual crystals to several sources of radiation?

- Natural isotopes in $\mathrm{Cu}$ and $\mathrm{Pb}$ and other materials

- Cosmogenics in Ge and other materials

- Cosmic muons

- Cosmic muon secondary neutrons

- $2 v$ double-beta decay

- $0 v$ double-beta decay

- Calibration sources

What is the collective response to these sources (i.e. self shielding effects)? What are the effects of proposed background cutting measures?

- Pulse-shape discrimination

- Simple segmentation 
- Segmentation with inferred position/multiplicity info

These questions have implications on $0 v$ double-beta decay $(2039 \mathrm{keV})$ and, at low energies, for dark matter. Validation of the results of this approach can be obtained by comparison of $2 v$ double-beta decay and calibration simulations to experimental data obtained in Phase 1 and Phase 2, and ultimately during Phase 3 itself.

The basics of the simulation, i.e. single-crystal energy deposition responses, have been developed previously using a code based on EGS4[Mil94]. More recently, a complete pulse simulation combined EGS4, a pulse formation code based on finite element analysis, and SPICE circuit simulation.

\begin{tabular}{|c|}
\hline 1. Radiation \\
Transport \\
and Charge \\
Deposition
\end{tabular}
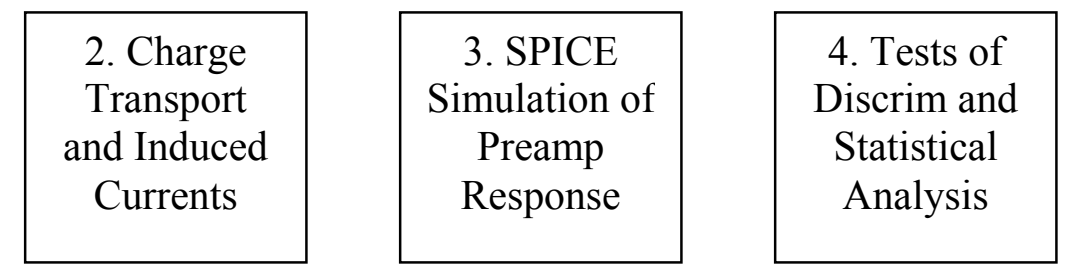

Figure 3-9. Complete pulse simulation approach.

Early development of pulse-shape discrimination was guided by this approach[Aal99b]. Comparisons between simulated and experimental data helped to elucidate those aspects of crystal properties and variations in electronic instrumentation causing large variations in observed pulse shapes. The observation of the large variations led to the development of a purely observational, self-calibrating pulse-shape discrimination approach.

Several of the results of interest have already been produced, for example the response of the pulse-shape discrimination method to internal and external sources of radiation, including $0 v \beta \beta$ decay. However, because multi-crystal depositions will also strongly identify signals as non-single-site, these results need to be expanded to include the complete Phase 3 apparatus responses.

The effects of segmentation have been addressed in a preliminary fashion by adapting the energy deposition location details in the EGS4 simulation output to allow evaluation of simple segmentation cutting. These elementary results are quite promising. It is anticipated that finer localization than the actual segmentation could be inferred from simple signal processing of adjacent-segment signals[Vet00]. However, to go beyond the simple segmentation-rejection results mentioned above, signals from real segmented detectors must be analyzed, in order to produce realistic refinement of the sensitivity calculation.

The missing components of the EGS4/single-crystal approach are the multi-crystal geometries and the ability to transport hadrons and score their energy depositions. These problems can be resolved by adopting GEANT4 for simulation. Besides allowing more detailed physics models, GEANT4 has the ability to accept geometry files created by the 
3-d solid modeling mechanical engineering software (SOLIDWORKS) used to design the Majorana detection hardware. Thus, extremely complete geometry files may be created, shared, and maintained with low effort.

Natural backgrounds generally include $\mathrm{U}$ and Th daughters, and ${ }^{40} \mathrm{~K}$. (See Appendix 2: Natural radioactivity decay chain data.) Of particular note are gamma rays from ${ }^{208} \mathrm{Tl}$ and ${ }^{214} \mathrm{Bi}$ owing to their energy sufficient to reach the $2039 \mathrm{keV}$ energy region-of-interest. Special cases exist like ${ }^{210} \mathrm{~Pb}$ and ${ }^{210} \mathrm{Bi}$ in the lead shielding, which pose a difficulty for low-energy measurements.

Cosmogenics in Ge are mainly ${ }^{68} \mathrm{Ge}$ and ${ }^{60} \mathrm{Co}$. Shorter-lived cosmogenics, such as ${ }^{58} \mathrm{Co}$ and ${ }^{56} \mathrm{Co}$ need to be simulated also because during early operation their presence can be used to validate the Monte Carlo and test electronic background-suppression methods. Cosmogenics in the copper should be non-existent due to the underground electroforming location and the underground storage of copper anode material.

Neutrons present a two-pronged problem: low energy fission or $(\alpha, n)$ neutrons $(<\mathrm{E}>\sim 1$ $\mathrm{MeV})$ and high energy muon-secondary neutrons $(<\mathrm{E}>\sim 100 \mathrm{MeV})$. For the high-energy neutrons, simulations will help optimize the shielding configuration of the experimental setup.

\subsubsection{Cryostat and Crystal}

The sensitivity goals of the Majorana Phase 3 instrument can only be realized if a stringent set of requirements is met regarding the physical form and immediate environment of the fiducial mass of germanium. With this in mind, a series of technical goals and requirements critical to the success of the Phase 3 instrument can be exposited for the cryostat and crystal mounting.

Balancing minimized crystal surface area against the feasibility of producing very large germanium diodes will drive the size of crystals in the Phase 3 array. The current experience of commercial germanium diode production facilities suggests a crystal size of $\sim 2.4 \mathrm{~kg}$ is practical while implementing segmented diode contacts. Monte-Carlo studies have been performed to determine the level of contact segmentation giving the best background rejection potential with the least added fabrication complexity. For example, figure 3-10 shows the acceptance fraction for important background sources as well as the signal of interest for various levels of axial contact segmentation.

These Monte-Carlo simulations, and discussions with commercial germanium diode fabricators, suggest a scheme of six azimuthal segments and two axial segments gives good efficacy while limiting manufacturing risk. This scheme is considered in the sensitivity estimates of the Phase 3 instrument and projections of instrumentation cost. 
To enable background rejection via pulse-shape discrimination, it is critical that the charge-integrating preamplifier of each diode contact have $<30 \mathrm{~ns}$ rise-time. To achieve this, the detector mounting technique must add little additional capacitance to that intrinsic to the diode contacts. Additionally, cross-talk due to inter-contact capacitive coupling must be minimized. This must be accomplished within the limited palette of materials known to be of sufficient radiopurity to be used close to the germanium crystal. A mounting and contact scheme, illustrated in figure 3-12, has been developed to meet these goals.

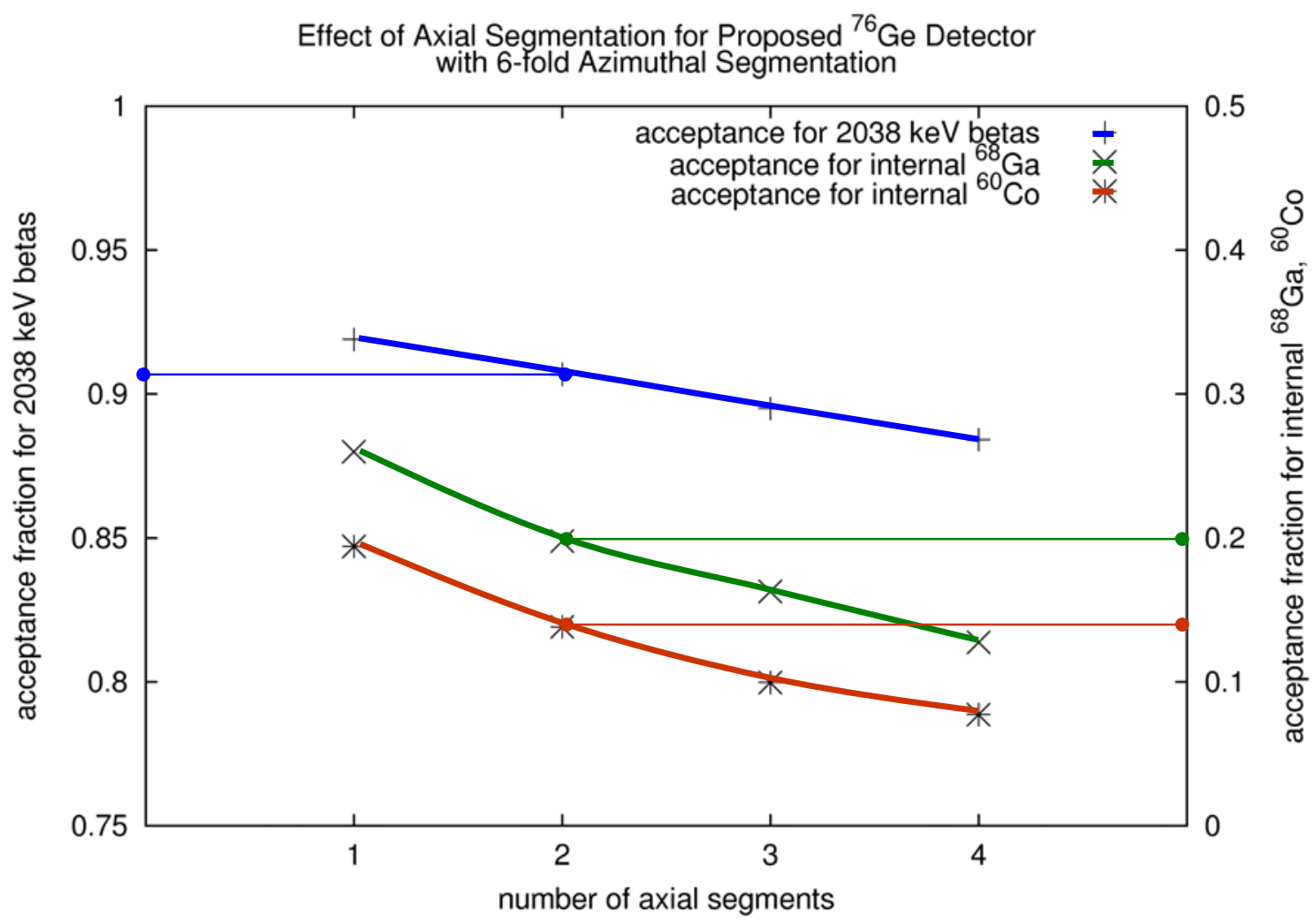

Figure 3-10. Acceptance fraction of a simple one-segment-hit cut vs. number of axial segments. Shown on left axis is acceptance fraction for events of interest. Shown on right axis is acceptance fraction for two important background contributors. 


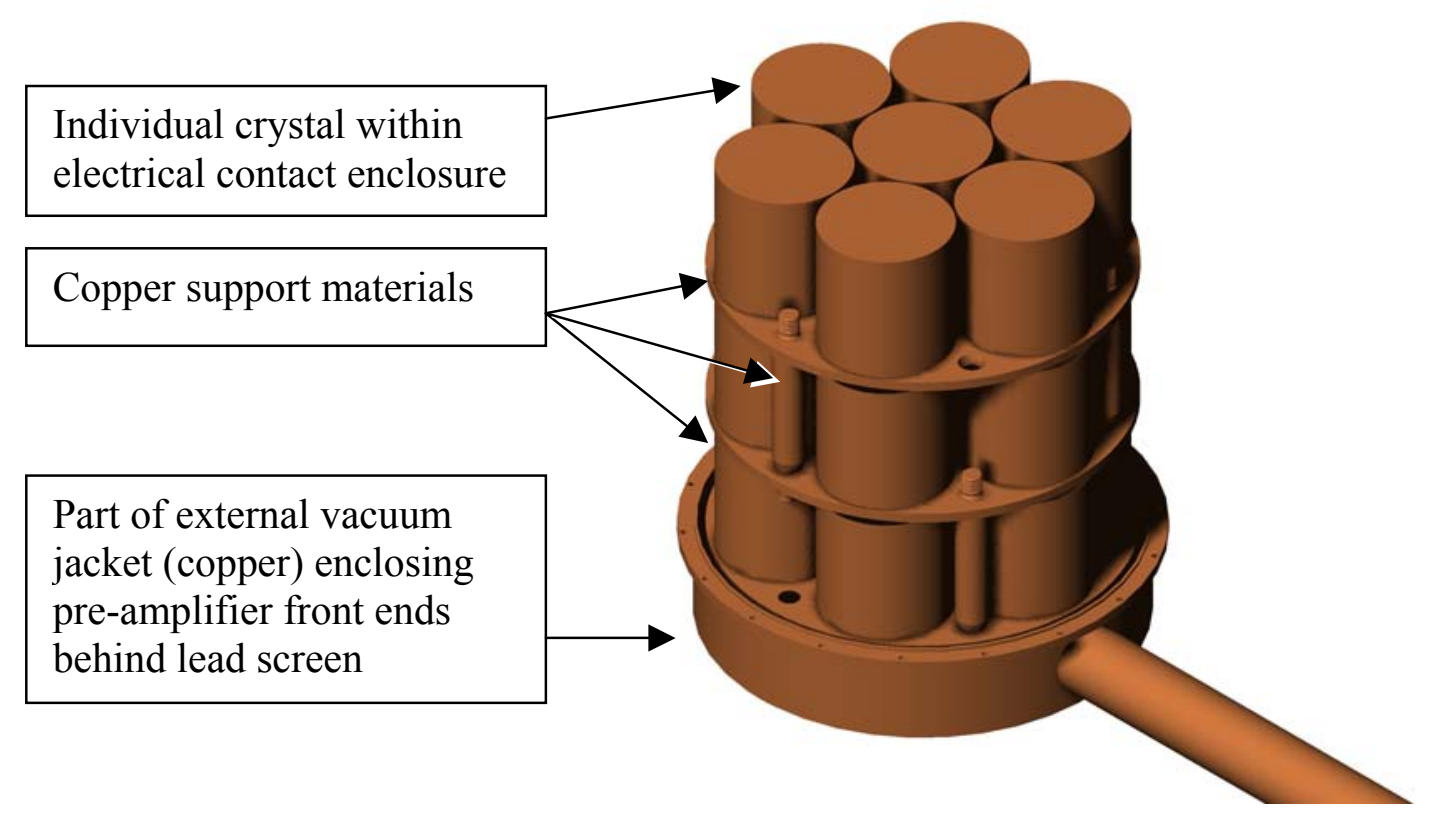

Figure 3-11. Highly-schematic view of close-packed arrangement of 21 germanium crystals inside a modular Phase 3 cryostat. Outer vacuum jacket is removed for clarity.

To maximize self-shielding effects, the crystals must be as closely-packed as possible while still providing the advantages of modular deployment. The background projections for the Phase 3 instrument depend critically on meeting current state-of-the-art goals as to the radiopurity of the support structures and cryostats housing the crystals. Additionally, the spectroscopic performance of the instrument depends on adequate cooling of the

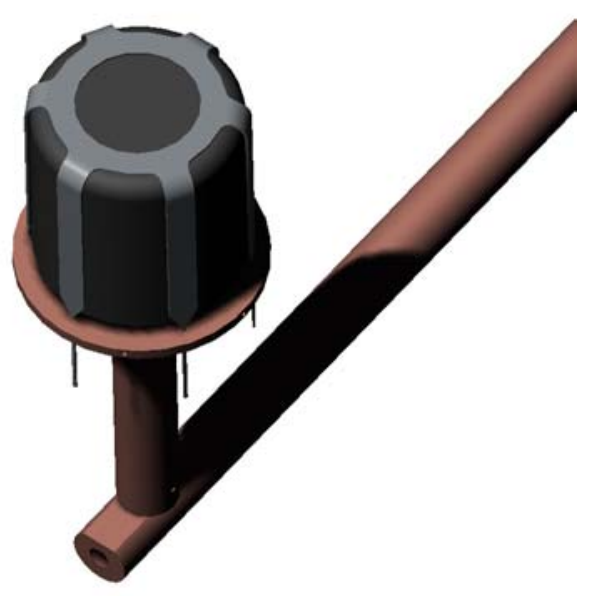
crystals as well as the electrical characteristics of the cryostat, mounting, and charge-sensing components. With these factors in mind, a baseline mechanical design has been developed, consisting of 10 modules with 21 crystals each. An internal view of one modular cryostat is shown in figure 3-11.

This arrangement allows the germanium crystals to be in close proximity to one another,

Figure 3-12. Rendered view of segmented Phase 3 maximizing their self-shielding detector outer support and electrical contact web potential. This design minimizes the amount of support material per gram of fiducial germanium

from about $2.1 \mathrm{~kg} \mathrm{Cu}: 2.1 \mathrm{~kg} \mathrm{Ge}$ in the previous IGEX experiment to about $7.5 \mathrm{~kg} \mathrm{Cu}$ : $50 \mathrm{~kg}$ Ge in the modular cryostat shown here. 
A series of detectors, based on the technology of ultra-pure electroformed cryostat components, have been produced by members of the collaboration for the IGEX collaboration and other uses. These deployments have demonstrated the cryogenic performance and long-term stability of this construction technique. Long-term measurements as part of the earlier IGEX effort have established the stringent limits on radiopurity of the electroformed copper support material, as exposited in the sensitivity calculation for the Phase 3 instrument[Bro95].

The crystal geometry, cryostat baseline design, and crystal mounting technique respond to the unique requirements mandated by the sensitivity and stability goals of the Phase 3 instrument with a combination of new design and proven techniques.

\subsubsection{Alternate Cooling/Shielding Investigation}

The standard method for cooling germanium detectors consists of the vacuum cryostat and copper cold-finger technique. As the number of crystals and associated electronics increases; however, the limitations of the cold finger and mechanical joints could result in insufficient cooling power at some level of heat load. On the other hand, this technique is well developed, well understood, and in the case of the IGEX detectors, was executed with cryostats of electroformed copper contributing only a very minor fraction of the background. It is therefore prudent to base plans on this technique early in the Majorana project, and to investigate alternative cooling configurations in parallel. One of the best features of high-purity, (or intrinsic) germanium detectors is the ability to remount them in other cryostats when so desired. There are three alternative cooling schemes that have been discussed with the detector manufacturers.

The favored alternative cooling technique of the detector manufacturers is the immersed vacuum chamber scheme. The operation of the detectors in vacuum prevents cosmogenically produced isotopes such as ${ }^{3} \mathrm{H}$ and ${ }^{14} \mathrm{C}$ from contributing to the low energy background of the detectors. In this configuration a tightly packed cluster of germanium detectors would be mounted on a low-mass frame of ultra pure material, inside a vacuum chamber fabricated from ultra pure copper. The chamber must allow high-temperature $(100 \mathrm{C})$ baking under a vacuum to clean all surfaces. When satisfactorily pumped, the entire chamber would be then immersed in a large tank of liquid nitrogen, and the detectors slowly cooled by black-body radiation from the crystals to the cold copper walls maintained at 77 degrees Kelvin. The vacuum would be maintained by a cryo-pump in a configuration that would have the radioactivity of the xeolite or charcoal well shielded from the detector chamber. In this configuration, the power dissipated in the first preamplifier stage could be adjusted to operate the fieldeffect transistors at an optimum temperature. This scheme should be the prime candidate for a research and development program conducted in parallel to the preparation of the first detectors and their installation in the primary conventional cryostats. Unfortunately, radiation makes a poor thermal coupling and therefore it must be demonstrated that the heat load in this multi-crystal array can be effectively removed by this technique.

The second is the direct immersion of naked germanium detector is in liquid nitrogen, as proposed by the GENIUS collaboration[Kla98]. They propose to lower an array of 
more than 300 detectors of approximately $3 \mathrm{~kg}$ each, directly into a large pool of continuously-purified liquid nitrogen. The proposed dimensions of the pool are large enough to utilize the liquid nitrogen itself as a shield against external radioactivity. Several detector experts do agree that liquid nitrogen is an excellent dielectric and that in the absence of moisture should not cause surface deterioration or leakage currents. In addition, quick, direct emersion of detectors at approximately 300 degrees Kelvin directly into liquid nitrogen at 77 degrees Kelvin will very likely not damage the detectors due to thermal stresses, because a layer of nitrogen gas will immediately form at the surface, severely slowing the transfer of heat to the detector. This is the effect that allows one to immerse a finger directly into liquid nitrogen for a short period of time without freezing it. The nitrogen purification system must be extremely efficient in preventing any moisture from freezing out on the surfaces. Prior to direct immersion, the surfaces must be heated in a high vacuum to eliminate all moisture. This is in principle possible, but technically difficult. Minute amounts of moisture frozen on the surface would be devastating to the proper operation of a detector. This problem will create a significant technical challenge.

There are several major negative aspects of this direct immersion configuration. The field-effect transistors (FETs), will not operate well at 77 degrees Kelvin. Therefore, either sufficient power must be dissipated locally, or else the FETs must be removed from the liquid nitrogen, resulting in a long cable length between the first and second stage of the preamplifier. The long cable length required to operate the FET outside of the liquid nitrogen bath will add significant capacitance, resulting in an increase in the energy threshold, as well as considerable degradation of the pulse rise time, and consequently the quality of pulse-shape discrimination (PSD), necessary to eliminate background. Failure to remove the transistors from the nitrogen may cause nitrogen to boil near the detectors and the transistors, which will raise the energy threshold due to microphonic noise.

Unfortunately, the direct immersion technique will not eliminate the main sources of background, namely, radioactivity internal to the crystal that is generated by cosmic-ray neutron interactions with the stable isotopes of germanium (Ge-70, 72,73,74,76). In addition, cosmic-ray muons will create Cherenkov light in the tank. Germanium behaves as an insulating dielectric but only in the absence of light. The Cherenkov light could cause significant surface currents that might harm the sensitive FET, cause pulses, or cause surface damage. Significant research and development will be required to explore and overcome technical challenges of the direct immersion technique.

The final technique utilizes a cold gas atmosphere surrounding the detectors, which is circulated from a chiller outside of the detector chamber. This gas would greatly improve the thermal coupling between the detectors and the chilling liquid. The chamber must be one that can be evacuated and could allow the detectors to be heated to about 100 degrees centigrade and pumped, to clean the surfaces and to completely eliminate moisture. The pressure and temperature of the gas would have to be carefully regulated to prevent arcing when the detectors are at high voltage. The advantage of this configuration is that it can be immersed in a large tank of continuously purified water or in a large volume of scintillator to act as a veto. 


\subsubsection{Shielding}

Assuming that all appropriate steps have been taken to eliminate sources of background radiation from the component parts of the detector and shielding materials, the limiting background for the proposed experiment comes from three potential sources: cosmic rays, experiment chamber walls and materials, and airborne radon. Each of these background sources can be mitigated with proper attention to detail.

First, primordial radionuclides in the walls and construction materials of the experimental area constitute the largest source of background radiation, and the easiest source to mitigate. A sufficiently designed and massive bulk shield composed of radiologicallyclean material, typically lead, surrounding the detector systems has been repeatedly demonstrated to effectively eliminate this source of background.

Radon daughter products are the most commonly-identifiable background source in lowbackground experiments. While elimination of radon from the sensitive region of the detectors is non-trivial, it can be done. This is most easily accomplished by enclosing the detector ensemble in an airtight container and pressurizing the internal volume of the container with a radiologically-pure gas, typically nitrogen boil-off from liquid nitrogen. The two important parameters are that the entire system consists of metal construction and that there is only one exhaust port for the pressurizing gas. Radon can permeate through plastics and rubber compounds, and if there is a leak anywhere in the enclosure, radon will migrate in through that leak regardless of the positive internal pressure.

Finally, cosmic-ray-related signals may provide the limiting background. Cosmic rays generate background via three separable mechanisms. The most common is via direct interactions in the detector and shield materials. An electronic anticosmic shield can be very effective in eliminating this source of background (but nothing is $100 \%$ absolutely effective). The second source of cosmic-ray-generated background is from interactions in the walls and other components of the experimental chamber that are not directly protected by the electronic anticosmic shield. This source of background typically manifests itself as a shower of high-energy neutrons, some of which can enter the detector ensemble without generating a blocking pulse in the electronic anticosmic shield, and that subsequently interact with the detector materials and components. This source of high-energy neutron-induced background can be mitigated through a proper choice and arrangement of bulk and electronic shielding materials. However, once again, this source of background cannot be completely eliminated. Last, and least of all, cosmic rays will interact with the detector materials themselves to generate spallation radioisotopes that decay long after the electronic blocking pulse has expired. These background events can only be mitigated by reducing the number of cosmic rays incident on the detector ensemble. The number of incident cosmic rays can only be reduced by increasing the depth of the overburden.

While primordial radioactivities in the chamber walls and radon in the air may vary substantially depending on location and rock composition, both are so insignificant in any case, that site selection should not be a consideration. Mitigation methods are required in 
all cases, and can be just as effective in all cases. However, the ultimately, limiting source of background, if all other sources are properly eliminated, are cosmic rays, and regardless of the efficiency of mitigation effects, they cannot be totally removed. Hence, the deepest site available always offers the potential for the lowest possible background.

The shielding of the Majorana Phase 3 apparatus is a critical although low-technology component. It reduces background counts from $\gamma$ rays in the experiment hall (from rock, construction materials, and possibly from shielding materials themselves), cosmic-ray muons penetrating the shielding, and cosmic-muon-induced neutrons. The strategy is to provide extremely low-activity material for the inner layer of $\gamma$-ray shielding. Around this will be bulk $\gamma$-ray shielding material of lower quality. Finally, outside this bulk shielding will be active muon veto detectors. Cosmic muon secondary neutrons may potentially require either great depth or an additional shell of muon detection, high-energy neutron moderation (e.g. lead), and hydrogenous moderator/ $\mathrm{Cd}-\mathrm{Ga}-\mathrm{B}$ absorber. This topic is currently under review.

\section{Inner shield}

Because of the extreme sensitivity of germanium detectors to $\gamma$ rays, and the extreme efficiency of the Majorana Phase 3 apparatus, $\gamma$ rays in the inner region of the shielding are important. While the special electronics discussed below will offer a degree of protection from $\gamma$ rays external to the detector (which have a high multiplicity), the direct approach is to use only materials with extremely low specific activity from radioactive isotopes. Fortunately, there are few sources of $\gamma$ rays above $1500 \mathrm{keV}$. Prime examples of potential high-energy contamination $\gamma$ rays are:

$2200 \mathrm{keV} \quad$ Hydrogen capture results from neutrons on hydrogenous material $2204 \mathrm{keV}$ $2505 \mathrm{keV}$ $2614 \mathrm{keV}$

$$
{ }^{214} \mathrm{Bi}, \mathrm{T}_{1 / 2}=19.9 \mathrm{~m} \text {, daughter of }{ }^{222} \mathrm{Rn}, \mathrm{T}_{1 / 2}=3.8 \mathrm{~d}
$$
Sum energy of ${ }^{60} \mathrm{Co}$ gamma rays from cosmogenic production in $\mathrm{Cu}$ ${ }^{208} \mathrm{Tl}, \mathrm{T}_{1 / 2}=3.05 \mathrm{~m}$, daughter of ${ }^{220} \mathrm{Rn}, \mathrm{T}_{1 / 2}=55 \mathrm{~s}$

Although the sum energy of the gamma rays from ${ }^{60} \mathrm{Co}$ exceed $2039 \mathrm{keV}(2505 \mathrm{keV})$, underground electroforming should prevent this spallation isotope from appearing in the $\mathrm{Cu}$ support structures. Assuming that there is essentially no hydrogen and/or no thermal neutrons within the inner shield, we can also assume that, as in the previous germanium experiments, we will observe no $2200 \mathrm{keV}$ hydrogen capture.

Thus, only the radon daughters ${ }^{214} \mathrm{Bi}$ and ${ }^{208} \mathrm{Tl}$ are of concern. If these are indeed only present due to $\mathrm{Rn}$, a few weeks after closing the cave and purging Rn they will no longer represent a concern at $2039 \mathrm{keV}$. On the other hand, if they are present because of contamination by a long-lived solid precursor, they could pose a significant problem, addressed below.

To qualify a material for inclusion in the inner area, an exceptional measurement technique is needed. The most elegant and effective method to qualify a material is to have used it in a previous experiment with similar, or even higher sensitivity to 
contaminants, with no observation of detrimental backgrounds. Unfortunately, this leads to a rather restrictive palette of materials with which to design the apparatus.

Although many materials have been used in previous germanium experiments, nearly all were in small quantities and thus can only be qualified for use in similar or smaller quantities. For instance, tens of grams of certain plastics have been used inside doublebeta decay germanium detectors in the past. We are therefore confident that we can continue their use in quantities in tens of grams.

A useful counter-example has been the germanium itself, which would show sharp alpha peaks in the $5 \mathrm{MeV}$ region if there were significant $U$ and Th contamination. In addition, the production process of the electroformed copper has been adapted to the results of numerous problems found in successive installations. In one particular experiment, an inner shield of $\mathrm{Cu}$ was produced in a hollow cylinder closed on one end (Marinelli geometry). This part was used as an inner shield for over 90 days and was therefore able to measure or limit concentrations of natural radioactivity in the $\mathrm{Cu}$.

$\begin{array}{lllccc}\text { Isotope } & \text { Chain } & \text { Activity } & \text { Conc }(\mathrm{g} / \mathrm{g}) & \text { Chain Conc }(\mathrm{g} / \mathrm{g}) \text { Relevant Daughter } \\ { }^{226} \mathrm{Ra} & { }^{238} \mathrm{U} & <25 \mu \mathrm{Bq} / \mathrm{kg} & <7.1 \times 10^{-19} & <2.1 \times 10^{-12} & { }^{214} \mathrm{Bi} \\ { }^{228} \mathrm{Th} & { }^{232} \mathrm{Th} & 9 \mu \mathrm{Bq} / \mathrm{kg} & 3.0 \times 10^{-22} & 2.2 \times 10^{-12} & { }^{208} \mathrm{Tl}\end{array}$

Because the chemical nature of the electroforming process tends to suppress elements in differing degrees, it is possible or even likely that the secular equilibrium of the $U$ and $T h$ natural decay chains (Appendix 2) were broken. Regardless of whether the lambda of the chain head or the inferred solid precursor isotope is appropriate for the calculation of

Table 3-3: A possible list of materials needed for the inner shields

\begin{tabular}{|c|c|c|c|}
\hline Component & Material & Quantity & Activity \\
\hline Ge Crystal & Enriched Ge & $500 \mathrm{~kg}$ & $\begin{array}{c}0.2 \mathrm{cts} / \mathrm{kev} / \mathrm{kg} / \mathrm{y} \\
\text { [Avi92] }\end{array}$ \\
\hline Cryostat & Electro Copper & $\begin{array}{c}\sim 10 \mathrm{~kg} / 21 \\
\text { crystal module }\end{array}$ & $\begin{array}{c}9 \mu \mathrm{Bq} / \mathrm{kg} \\
{[\mathrm{Bro} 95]}\end{array}$ \\
\hline Inner $\mathrm{Pb}(10 \mathrm{~cm})$ & Old Pb & $<1$ ton & $\begin{array}{c}<9 \mathrm{mBq} / \mathrm{kg} \\
\text { [Mil94] }\end{array}$ \\
\hline Inner electronics & Various & $\mathrm{O}(100 \mathrm{~g})$ & $\begin{array}{c}\text { Below IGEX } \\
\text { MDC } \\
\end{array}$ \\
\hline $\mathrm{Cu}$ roof support & Electro Copper & $\sim 10 \mathrm{~kg}$ & $\begin{array}{c}9 \mu \mathrm{Bq} / \mathrm{kg} \\
{[\mathrm{Bro} 95]}\end{array}$ \\
\hline Outer $\mathrm{Pb}(20-30 \mathrm{~cm})$ & Commercial Pb & $\sim 30$ tons & NA \\
\hline Veto detectors & $\begin{array}{c}\text { Plastic } \\
\text { Scintillator }\end{array}$ & $10 \mathrm{~m}^{2} \times 10 \mathrm{~cm}$ & NA \\
\hline
\end{tabular}

grams per gram, the relevant quantity is the measured specific activity, which translates into the specific activity of the observable gamma rays: at or less than about $10^{-8} \mathrm{~Bq} / \mathrm{g}$. 
Another material qualified in previous experiments is lead. Unfortunately, while we can make clean copper in any quantity or shape we require, sources of lead are opportunistic and hence problematic. The sources of lead used include Doe Run mine, Johnson Matthey/Cominco Inc., 150 year old German ingots, 450 year old Spanish galleon ballast, and 2000 year old Roman anchors. Old lead is preferred because the chemical cleaning that occurs in the smelting process (akin to zone refining) cannot eliminate ${ }^{210} \mathrm{~Pb}\left(\mathrm{~T}_{1 / 2}=\right.$ $22.3 \mathrm{y}$ ), a lower link in the ${ }^{238} \mathrm{U}$ chain.

The ${ }^{210} \mathrm{~Pb}$ itself beta decays, emitting a $46.5-\mathrm{keV}$ gamma ray. The decay product ${ }^{210} \mathrm{Bi}$ beta decays (essentially no gamma rays) and produces a bremstrahlung spectrum out to $1162 \mathrm{keV}$. The final decay before stable ${ }^{206} \mathrm{~Pb}$ is ${ }^{210} \mathrm{Po}$. Alphas emitted by ${ }^{210} \mathrm{Po}$ at 5304 $\mathrm{keV}$ are visible in a sharp peak if exposed to the active surfaces of a germanium detector. Of these, only the ${ }^{210} \mathrm{Po}$ alpha represents an issue for double-beta decay at $2039 \mathrm{keV}$. This alpha has been successfully eliminated by careful detector construction. The others do represent an issue for dark matter.

Besides controlling particulate deposition (both Rn daughters and ordinary dust), the best solution for the low-energy problems of common $\mathrm{Pb}$ is to use either screened $\mathrm{Pb}$ or electroformed $\mathrm{Cu}$ in the innermost centimeters of the shield.

\section{Outer shield:}

The outer shield protects the detectors from gross environmental $\gamma$ rays in the rock, the construction materials, and other shielding materials. The perfect example is a pair of 1.05 $\mathrm{kg}$ natural germanium detector operated for several years in a $\mathrm{Pb}$ shield of about 1 meter cubed. The minimum thickness of $\mathrm{Pb}$ was about $30 \mathrm{~cm}$ of well-stacked bricks (no cracks). These were arranged to allow reasonable access to the detectors, and no observable external $\gamma$ rays were detected in the equivalent of 1 year of uninterrupted operation.

Also in this example, a 10 -cm-thick $4 \pi$ plastic scintillator was constructed plastic instrumented sparsely with photomultiplier tubes. It was immaterial that the energy response/energy threshold of the plastic detectors varied substantially from end to end: the threshold was well below the muon through-peak of a perpendicular transversing muon. This active shield in some experiments was recorded as an independent signal, allowing an independent measure of the health of the subsystem.

Draft

Page 44 
Given that the area of the muon veto system is a few $\mathrm{m}^{2}$, the muon rates expected in any of the available underground labs can be effectively cancelled. Other processes are of more immediate concern, and cannot be completely addressed without some experimental testing.

One concern is neutron transmutation of germanium into long-lived isotopes. As an example, we take the neutron spectra at zero mwe of Hess and compare to that of Gaitskell

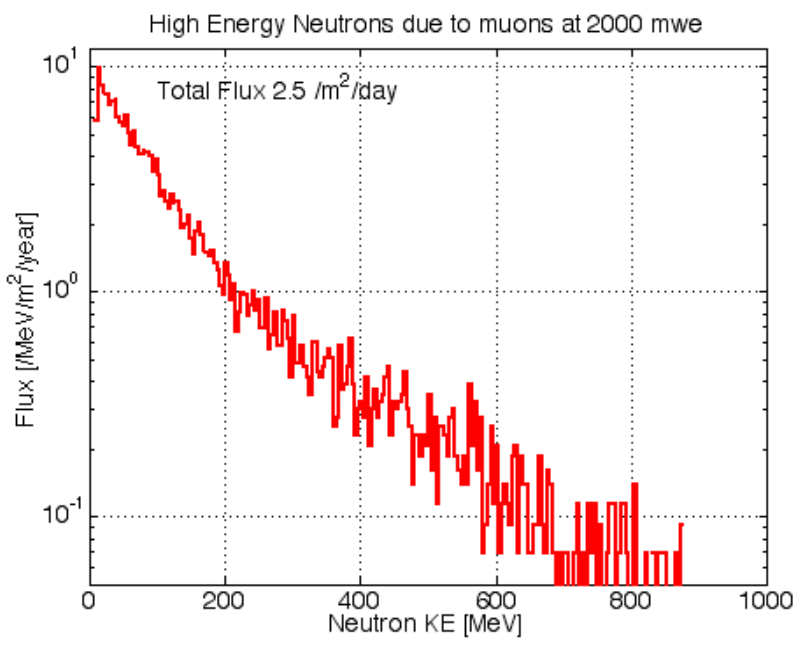

Figure 3-13 Computed neutron spectrum at 2000 mwe. Below this depth, the spectral shape is similar and the flux is reduced. [Gai01]

[Gai01]. At $1 \mathrm{MeV}$, Gaitskell

calculates $10 / \mathrm{MeV} / \mathrm{m}^{2} / \mathrm{yr}$ where Hess reports $3 \times 10^{11} / \mathrm{MeV} / \mathrm{m}^{2} / \mathrm{yr}$, while at $100 \mathrm{MeV}$ Gaitskell calculates $2 / \mathrm{MeV} / \mathrm{m}^{2} / \mathrm{yr}$ and Hess reports $5 \times 10^{7} / \mathrm{MeV} / \mathrm{m}^{2} / \mathrm{yr}$. Since the cross sections for neutron spallation become significant between $20 \mathrm{MeV}$ and $100 \mathrm{MeV}$ for isotopes such as ${ }^{60} \mathrm{Co}$ and ${ }^{68} \mathrm{Ge}$, we can conservatively estimate that the spallation rate at 2000 mwe or greater will be about $10^{7}$ smaller underground, or that spallation of the germanium during the entire 10 year operation will be one ten-thousandth of a single day above ground.

Elastic scattering of neutrons is another concern. While the rate of neutrons reaching the detector with sufficient energy to create a recoil event registered at $2039 \mathrm{keV}$ is computed (again [Gai01]) to be only around 2-3 per year per whole Majorana Phase 3 system, some consideration to the rejectability of the signals should be given, and has not to date.

Another concern is neutron inelastic scattering on detector, structural, and

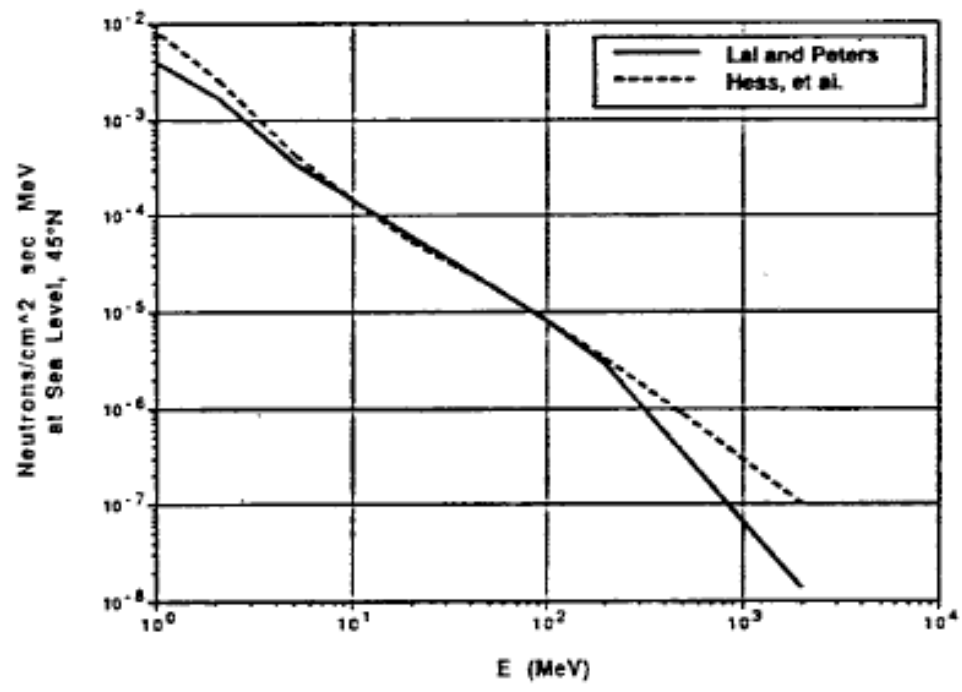

Figure 3-14 Neutron spectra used to calculate surface spallation rates. [Avi92] 
shielding materials. These can be greatly reduced by use of aggressive shielding or veto techniques, but the magnitude of this contribution was unidentifiable in previous experiments and a limiting value has not yet been determined from computations.

We can conclude at this time that a shield capable of eliminating external gamma-rays is quite achievable, as is a direct muon veto system. However, additional consideration must be given to the need and optimum design of a shielding or veto system for muoninduced neutrons.

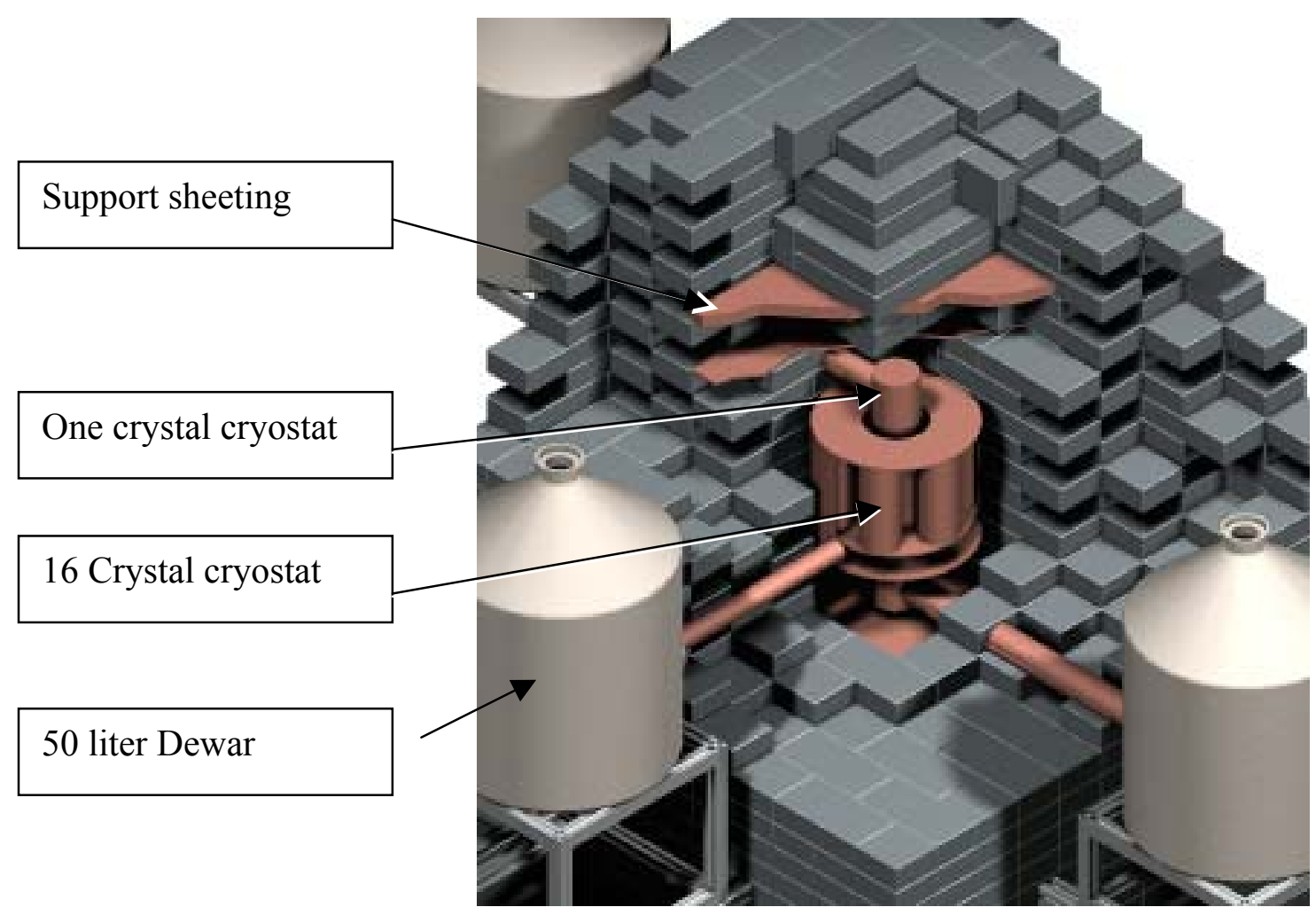

Figure 3-15. Detail from drawing of Phase 2 detectors: Plan to support lead above detector cavity. The outer jacket of the 16-crystal cryostat is removed for clarity.

\section{Mechanical Engineering}

The shield has been designed to use the minimum amount of mass needed to shield a large number of detectors. If the crystals were each shielded with a $\mathrm{Pb}$ cube of $30 \mathrm{~cm}$ (about $280 \mathrm{~kg}$ ), about $60,000 \mathrm{~kg}$ of $\mathrm{Pb}$ would be required for the total experiment. To reduce the need for low background materials, reduce the footprint of the experiment, and allow multi-crystal gamma-ray depositions (for effective identification and suppression), the entire detector mass is shielded with one $\mathrm{Pb}$ layer $30 \mathrm{~cm}$ thick. The inner cavity occupied by the detector is about $100 \times 70 \times 70 \mathrm{~cm}$. The outer dimensions are about 160 $\mathrm{x} 130 \mathrm{x} 130$. Thus the total mass is about $25000 \mathrm{~kg}$ of Pb.

This entire mass must rest on a muon veto which can directly carry the load. In addition, the mass over the detector area $(100 \times 70 \times 30 \mathrm{~cm})$ must be supported above the detectors 


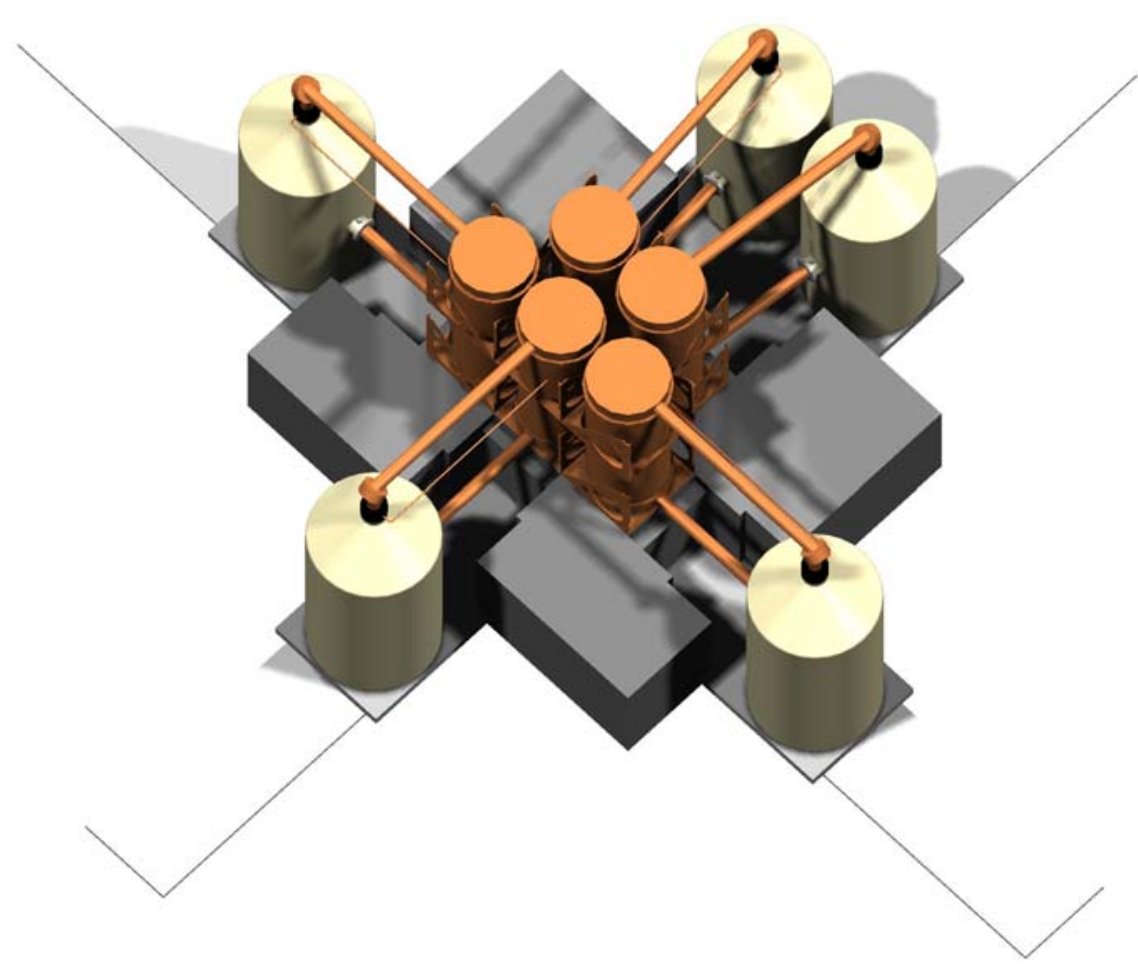

Figure 3-16. Layout of 10 21-crystal modules showing monolith slides. The lead shielding is cut short in this drawing to expose the inner structure.

with great confidence. Fortunately, lead bricks are somewhat self supporting, but the worst case is that $210 \mathrm{~kg}$ must have an ultra-clean support material.

The strategy now under review is the use of electroformed copper to support the lower portion of the shield, with a common OFHC support midway up in the lead. This approach is being adapted from the plan for the Phase 2 system.

Supporting the lead over the $30 \times 30 \mathrm{~cm}$ Phase 2 detector cavity required a unique approach. The standard approach would be to simply place a sufficiently thick plate of support material, in this case copper, across the cavity. However, the plate would need to be electroformed because the high background levels that exist in commercia-grade copper. Electroforming a plate of sufficient thickness was also considered too difficult. Therefore, the following design was suggested and analyzed.

Support for the lead above the $30 \times 30 \mathrm{~cm}$ detector cavity is accomplished with two copper plates. The cavity is overlaid with a $0.5 \mathrm{~cm}$ thick electroformed copper plate. This plate achieves two things. First, it provides background shielding for the detector. Second, it provides the structural support for the first layer of lead bricks over the cavity. A $5 \mathrm{~cm}$ thick layer of lead bricks covers the electroformed copper plate.

The additional layers of lead would be supported by a second plate of commercial type 101 copper, about 1-cm thick. Design analysis shows that these plates will support up to 
$40 \mathrm{~cm}$ of lead over the cavity with an allowable deflection of $<2 \mathrm{~mm}$. This design also keeps the background exposure to the detector minimal.

Additional support for Phase 3 can be provided at low cost by a 'strongback' arrangement of thin copper pieces. This design is currently under review.

The cave geometry has been chosen to minimize shielding and maximize detector selfshielding and multi-crystal gamma-ray detection. However, it will be necessary to access the detector modules for upgrades and repairs. A design is needed which facilitates access, maintenance, and the addition of 10 modules.

The current plan is shown in figure 3-17. The white rectangles behind the Dewars are slides which facilitate the hand or motor operated hydraulic removal or insertion of a detector monolith. This design allows the lead cave to be constructed independent of the germanium detector progress. The modules may then be installed individually during the construction and commissioning phase of the experiment. Periodic maintenance of a single detector crystal may be conducted without disruption of the entire apparatus.

These plans show that the basic detector unit is a plug of shielding with a large (50 1) Dewar on one side and two detector modules on the other. This unit (figure 3-17) may be emplaced or removed by hydraulic-powered sliding. The order of module insertion can

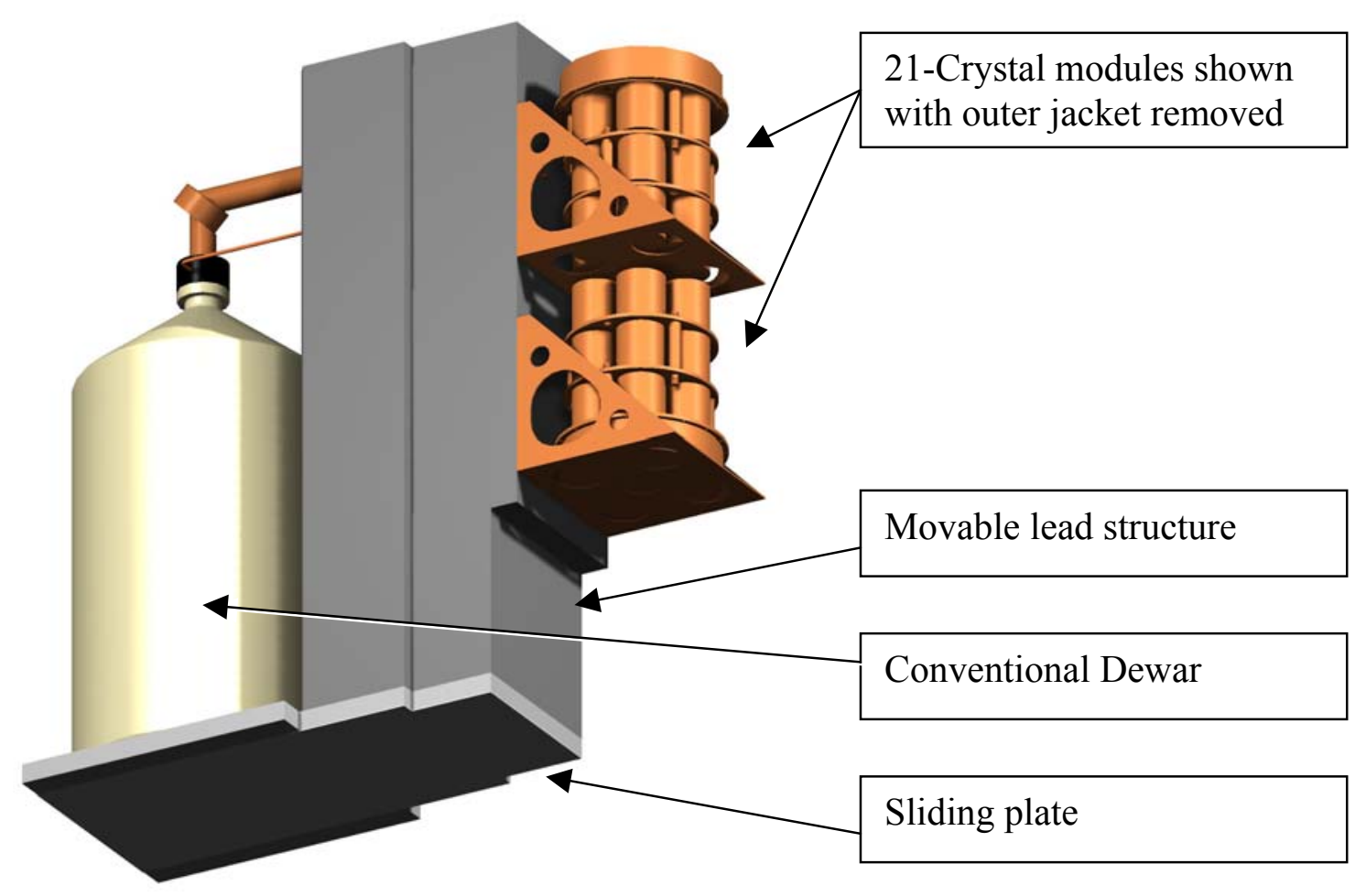

Figure 3-17. View of a movable detector monolith. Two 21-crystal modules will be cooled by a single LN Dewar, upper module by dipstick arrangement, the lower by integral connection to the Dewar. 
be either (1) each sliding component can be filled with two 21-crystal modules in turn, or (2) that all lower modules can be inserted then all upper modules.

\subsubsection{Electronics and Data Acquisition}

The instrumentation of the Majorana Phase 3 apparatus is driven by the need for low power dissipation and radiopurity while maintaining fast rise-time performance to support pulse-shape discrimination and a negligible contribution to detector resolution. Specialized front-end modules near the crystal contacts are combined with chargeintegrating preamplifiers and digital processing of the preamplifier output pulses to achieve this goal.

To achieve the best current-pulse shape fidelity and lowest-noise operation, it is necessary to amplify the current pulse evolved in a germanium ionization spectrometer without introducing additional capacitance. Any lead-length capacitance added to the irreducible capacitance of the detector electrode under observation reduces the magnitude of the signal by charge-sharing, reducing the signal-to-noise ratio of the induced current pulse relative to thermal fluctuations and other noise sources. The traditional, and very successful, solution to this problem is to locate the first-stage FET of a charge-integrating preamplifer very near the detector electrode under observation. This front-end FET gives considerable voltage gain and provides an output impedance of $\sim 100 \mathrm{Ohms}$, suitable for driving a connection to the rest of the preamplifier circuit. The remaining part of the charge-integrating preamplifier can be located at some distance from the detector electrode, allowing the detector to be shielded from radioactivity in the preamplifier materials.

Members of the collaboration, while working on the IGEX experiment, have explored optimizations of the front-end electronics to further reduce background contribution while preserving excellent signal fidelity[Aa199a]. Further optimizations of the electronics located near the detector electrodes are planned, with multi-chip module technology under consideration. The techniques of multi-chip module construction may allow more sophisticated analog electronics to be implemented near each detector electrode without sacrificing low background. This has the potential to reduce the number of connections entering the cryostat from the typical four found in most commercial setups (FET source, FET drain, feedback voltage, test pulse injection). With shared power connections and charge-integration completely inside the cryostat, each electrode could have as few as one connection to the outside.

After the recovery of an induced-current signal via charge integration, the signal is ready for further processing. Recently digital spectroscopy hardware platforms have become commercially available for this task. After digitizing the output of a charge-integrating preamplifier, all subsequent processing is done in the digital domain. This gives remarkable flexibility in matching filter and shaping parameters to the characteristics of the detector. This allows very low energy thresholds when compared to traditional analog shaping amplifier performance. Additionally, these new hardware platforms make available the digitized information necessary to do pulse-shape discrimination for 
background rejection based on interaction multiplicity. Other useful analyses, such as pulse-shape discrimination for the localization of single-site interactions are possible.

In the Phase 3 instrument, preamplifier output pulses will be digitized by commercial digital spectroscopy modules, the DGF4C series, manufactured by X-ray Instrumentation Associates. These CAMAC-based modules capture all energy, timing, coincidence information, and integrated current signals for subsequent pulse-shape discrimination. A binary data stream, defined by the DGF4C firmware, is sent in buffered blocks to the data acquisition and control system.

The electronics system will also include a computer-controlled a high voltage bias supply system for both the HPGe detector array and the phototubes of an anticoincidence shield. Separate, conventional, instrumentation will derive veto signals from an anticoincidence shield. These veto signals are combined into the DGF4C data stream via a Global Second-Level Trigger (GSLT) input, providing a timestamp in the data stream for veto firing.

By using a combination of highly-evolved low-background electronics near the detector and commercial modules for digitization and energy processing, the technical capabilities of the detector instrumentation are maximized (pulse-shape discrimination, low radioactivity) while minimizing engineering risk and cost.

The time-stamped data stream from each DGF4C module is sent to a control computer, where an event data model utilizing the ROOT framework (http://root.cern.ch) is populated and the ROOT tree subsystem is used for local data storage and serving the data stream to other locations for further analysis. State-of-health data is continuously monitored for variables such as cryostat temperature, ambient radon levels, detector segment leakage current, etc., and will be logged locally and served to other locations for further analysis and real-time monitoring.

The software control system will implement the various operating modes necessary for the experiment operation. These include normal operation, calibration, and various diagnostic functions. Members of the collaboration have many years of experience in developing sophisticated control systems for remote, unattended, gamma spectroscopy systems, and will be able to apply developed techniques to the control and monitoring of the Majorana Phase 3 experiment. Further discussion of this topic appears in the next section, "Computer Science Infrastructure".

\subsubsection{Computer Science Infrastructure}

The classical functions of nuclear data acquisition have changed in the last twenty years: it is now routinely possible to acquire many types of state-of-health data $(\mathrm{SOH}$ : temperatures, pressures, currents, and so forth) and to produce many products besides raw data, ready for post-processing. The structure of a system for sharing, processing, reporting, and archiving the data and data products can provide a new level of quality and ultimately confidence in results at a minimal cost. The raw data, results and all experiment $\operatorname{logs}$ will be electronic and shared freely within the collaboration. 
Raw data will originate from the several underground facilities housing the Majorana experiment hall, the electroforming laboratory, and other instrumented spaces. The types of raw data include:

- Time-stamped germanium pulse waveforms

- Time-stamped veto signals

- Periodic SOH data readings (frequency $\sim 1 / \mathrm{min}$ )

○ Experiment hall

- Oxygen levels

- Detector temperature/leakage current

- Room temperature

- Radon level

- LN levels

- LN purge gas flow

- Electronics temperature

- Barometric pressure

- Airborne particle concentration

- HV status

- Motion sensors

- Shielding location sensors

- Power: I and V

- Electroforming lab

- Radon level

- Airborne particle concentration

Data products also originating within the underground location include email alerts when state-of-health parameters exceed preset bounds. In addition, it is possible to log and share all electronic events from the acquisition system as well as human-generated log entries.

This stream of raw data can be forwarded to all appropriate parties and logged locally in case of communications interruption, but to insure uniform data quality and provide storage/archival, the data will be sent to a central data hosting facility. Each data message will be logged in a chronological way (and categorized by module, etc.) and described in a cost-effective data base.

A web interface to this data base is an effective way to quickly look for alerts and correlate them with anomalies in $\mathrm{SOH}$ data using helper applications for viewing each type of file. This allows human SOH monitoring to occur at all collaboration institutions at all times of day and takes great advantage of the global distribution of collaboration members. A prototype of this system is currently running at PNNL.

Of course, this monitoring of periodically-transmitted files is not instantaneous: if data is sent in, say, 1-day increments, SOH monitoring will be after the fact. For this reason, viewer applications are under development that will allow effectively instantaneous 
inspection of a live stream of $\mathrm{SOH}$ data. This will be quite useful for setup, calibration, and maintenance of the apparatus and the related systems.

The receipt of the raw data in a central data hosting facility also implies that unattended auto analysis can take place with the resulting data product distributed. While detailed, parallel analyses will take place in various locations, it is important to have a reasonably high-level and totally uniform method of gauging basic status of the acquired data. It would be prudent to have both analysis of germanium pulses (perhaps into simple 1dimensional histograms) and analysis of $\mathrm{SOH}$ parameters to take advantage of statistical failure prediction models.

There is a mature body of mathematical/statistical techniques to characterize system reliability and predict system failure. These techniques effectively model the probabilistic dependence structure of $\mathrm{SOH}$ measurements and use these models to formulate mean-time-to-failure estimates with associated uncertainties, component failure predictions and preemptive failure detection, and they provide defensible guidance on where to focus engineering efforts to improve system reliability. These techniques include general stochastic processes (e.g., Markov chains and processes) and time-series analysis, reliability models, experimental design and accelerated-life testing methods.

\subsubsection{Analysis}

Majorana is not simply a volume expansion of previous experiments, such as IGEX. It must have superior background rejection. Because it has been conclusively shown that the limiting background in at least some previous experiments has been cosmogenic activation of the germanium itself, it is necessary to mitigate those background sources. Cosmogenic activity fortunately has certain factors which discriminate it from the signal of interest. For example, while $0 v \beta \beta$-decay would deposit $2 \mathrm{MeV}$ between two electrons in a small, perhaps $1 \mathrm{~mm}^{3}$ volume, internal ${ }^{60} \mathrm{Co}$ decay deposits about $318 \mathrm{keV}$ (endpoint) in $\beta$ energy near the decaying atom, while simultaneous $1173-\mathrm{keV}$ and $1332-\mathrm{keV} \gamma$ rays can deposit energy elsewhere in the crystal, most probably both in more than one location, for a total energy capable of reaching the $2039 \mathrm{keV}$ region-of-interest. A similar situation exists for internal ${ }^{68} \mathrm{Ge}$ decay. Thus deposition-location multiplicity distinguishes double-beta decay from the important long lived cosmogenics in germanium. Isotopes such as ${ }^{56} \mathrm{Co},{ }^{57} \mathrm{Co},{ }^{58} \mathrm{Co}$, and ${ }^{68} \mathrm{Ge}$ are produced at a rate of roughly 1 atom per day per kilogram on the earths surface. Only ${ }^{60} \mathrm{Co}$ and ${ }^{68} \mathrm{Ge}$ have both the energy and half-life to be of concern.

To pursue the multiplicity parameter, two approaches are possible. First, the detector current pulse shape carries with it the record of energy deposition along the electric field lines in the crystal; crudely speaking, the radial dimension of cylindrical detectors. This information may be exploited through pulse-shape discrimination, as described below. Second, the electrical contacts of the detector may be divided to produce independent regions of charge collection, the detector segmentation scheme described earlier. 
By segmenting the inner contact into two (axial) parts and the outer contact into 6 (azimuthal) parts, as was described earlier in Section 3.4.2, multiplicity data can be obtained.

The Monte-Carlo simulation data set shown in figure 3-18 is based on this configuration and shows that internal highly-multiple backgrounds like ${ }^{60} \mathrm{Co}$ can be strongly suppressed at $2039 \mathrm{keV}$. The internal ${ }^{60} \mathrm{Co}$ modeled in the figure is produced by cosmic-ray neutrons during the preparation of the detector, accumulating after the last crystal-growth step. Its elimination by segmentation and pulse-shape discrimination is crucial. Beyond this simple segmentation cut, it may be possible to use the signals derived from segments seeing no net charge, adjacent to a segment seeing net charge, to locate a single-site deposition in the axial and azimuthal coordinates of the crystal or to distinguish a singlesite deposition from a multiple one. The results of the initial Monte-Carlo simulation described above predict a discriminator with an acceptance for $0 \vee \beta \beta$-decay events of $90.7 \%$. The predicted acceptance for internal ${ }^{60} \mathrm{Co}$ events is only $13.8 \%$.

Pulse-shape discrimination (PSD) is another way to gain sensitivity to interaction
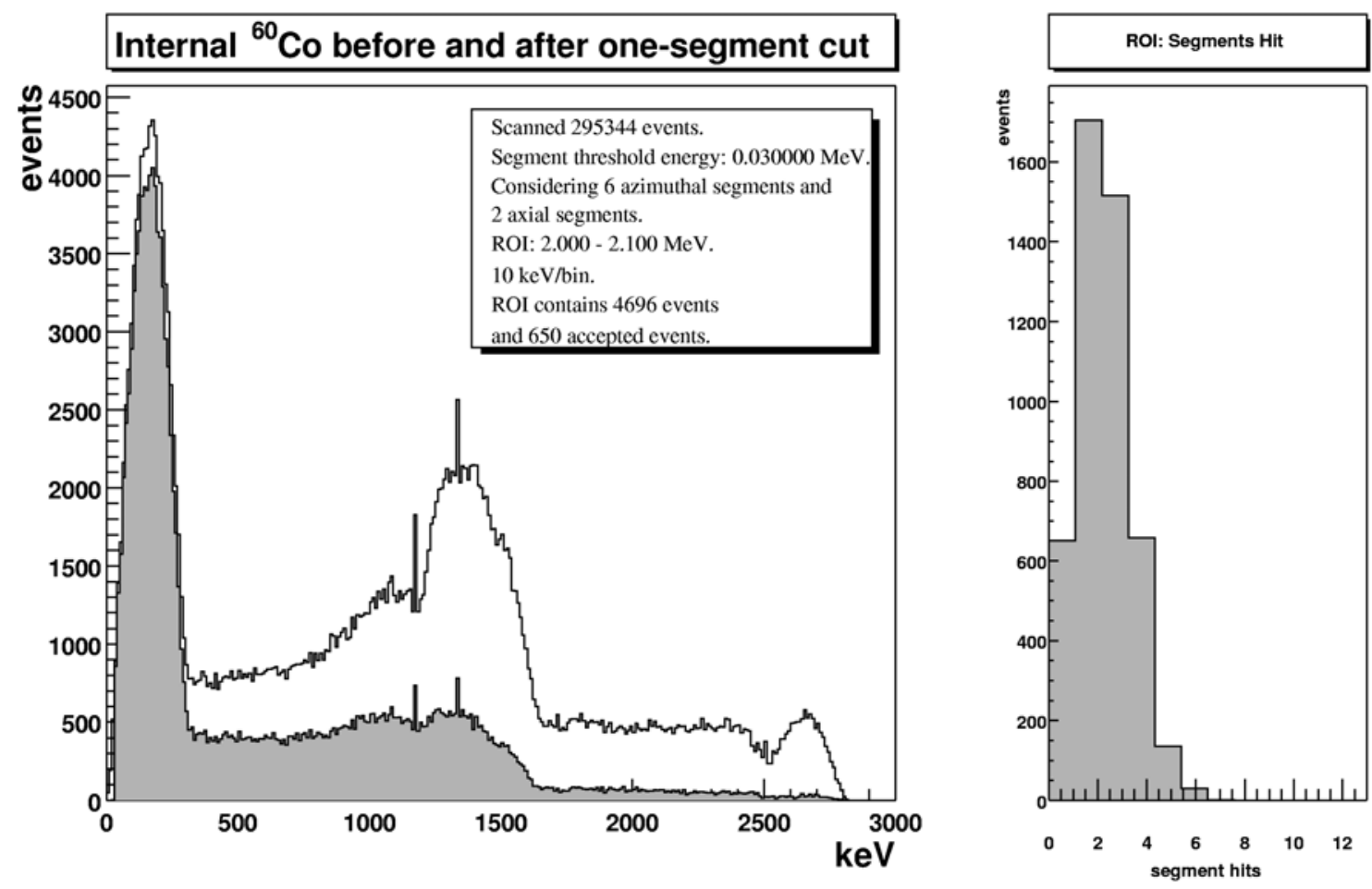

Figure 3-18. Monte-Carlo simulation of internal ${ }^{60} \mathrm{Co}$ background. Left shows a spectrum before and after a one-segment-only cut is applied. Right shows histogram of number-ofsegments-hit for events falling in 2.0-2.1 MeV ROI.

multiplicity, and works by examining the digitized current pulse, as presented by the output of the charge-integrating preamplifiers. Several years of research at PNNL and the University of South Carolina have produced a new PSD technique lacking many of the disadvantages of previous methods. 
Early research showed that common methods based on comparison of pulse-shapes to libraries or basis sets of calculated single-site pulses have disadvantages. Comparing each pulse to even a small library of template pulses is computationally intensive. More problematic is the fragility of templates or libraries of calculated pulses in the face of normal variations in experimental conditions. These variations could include changes in operating voltage, differences or inhomogeneities in minority carrier concentration, and variations in the alignment and operating parameters of different preamplifiers.

These problems are avoided with the parametric, self-calibrating, PSD technique recently developed. Using only a short calibration data-set, easily acquired with conventional lowlevel calibration sources, the discriminator is optimized for each detector/electronics setup. This calibration is fast, allowing the discriminator to be re-optimized frequently to account for any changes in the operating conditions. Using only a small number of parameters extracted from each pulse, this technique has modest computational requirements, allowing analysis to be rapid.

Monte-Carlo data suggest two types of experimental data are useful in quantifying the

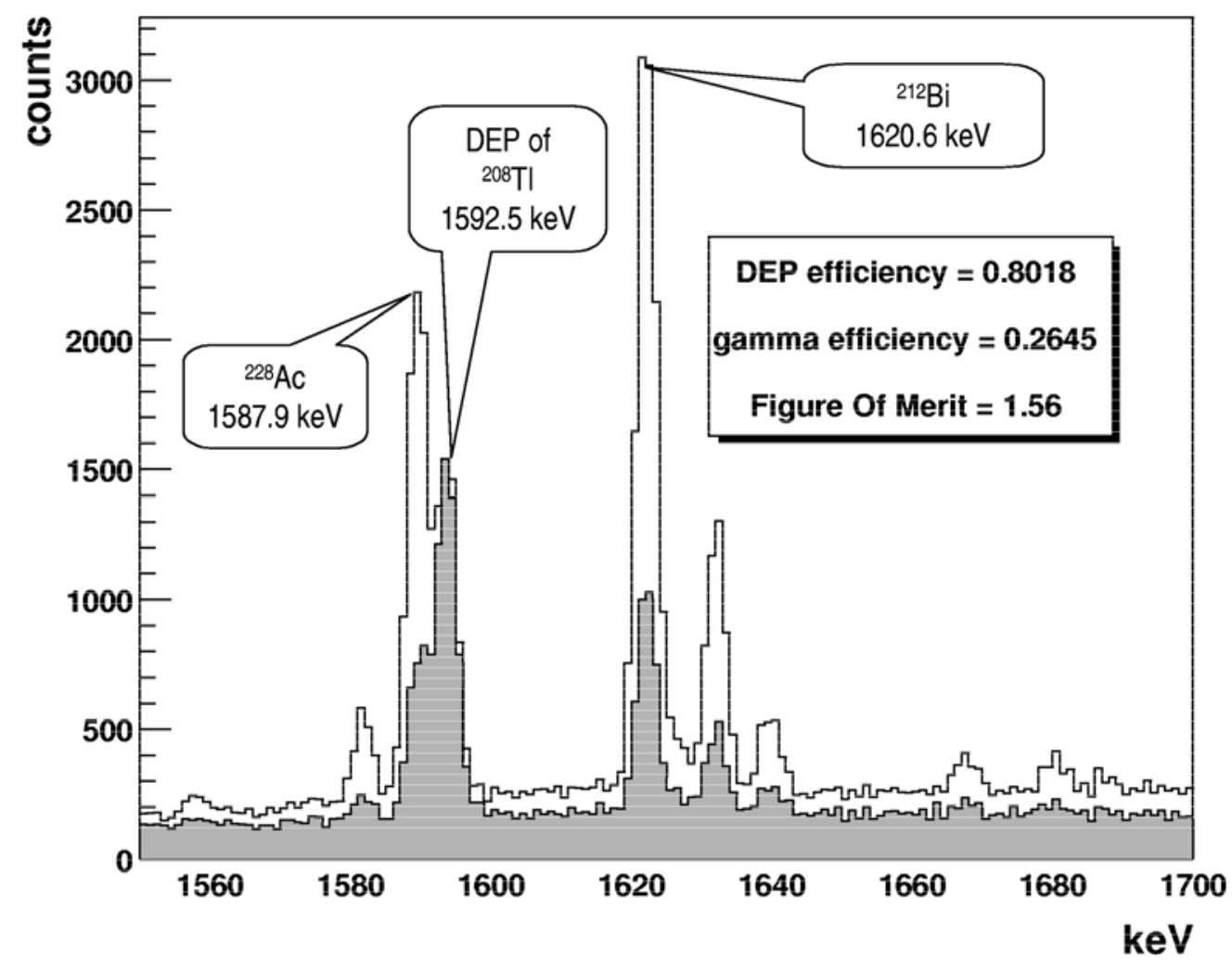

Figure 3-19 Portion of double escape peak (DEP) spectrum before (white) and after (gray) application of PSD discriminator. Residue (gray)_spectrum has been scaled for equal DEP intensity before and after discrimination.

efficacy of such a discriminator. Events in the full-energy peak of the 1620.6-keV line from ${ }^{212} \mathrm{Bi}$ were calculated to have an event multiplicity slightly lower than that of the 
expected internal cosmogenic backgrounds near $2 \mathrm{MeV}$. Thus these events are a conservative source of background-like events. Events in the $1592.5-\mathrm{keV}$ double-escape peak (DEP) of the $2614.5-\mathrm{keV}$ line from ${ }^{208} \mathrm{Tl}$ are calculated to have an event multiplicity nearly identical to those from $0 v \beta \beta$-decay. Thus these events are a good source of signallike events.

Applying the new PSD discriminator to an experimental data-set gives the result seen in figure 3-19. The white spectrum is the original data, while the gray spectrum is the result of applying the discriminator. The features of interest are the initial and final peak areas for the two types of events. For the DEP events, the discriminator yielded an acceptance fraction of $80 \%$, and the gray spectrum is normalized by dividing out this fraction. This shows the DEP intensity as the same for the two data sets and facilitates visual analysis of the relative reduction in the intensity of the gamma peak. For an experiment governed by Poisson statistics, the sensitivity scales as the square root of the background, while also scaling linearly with detection efficiency. This suggests defining a figure-of-merit (FOM) as

$$
F O M=\frac{\varepsilon_{\beta \beta}}{\sqrt{\varepsilon_{b k g}}}
$$

where $\varepsilon_{\beta \beta}$ is the efficiency of a discriminator for $0 v \beta \beta$-decay events and $\varepsilon_{\mathrm{bkg}}$ is the efficiency for background events. This FOM is then the multiplicative factor increasing or decreasing the half-life limit of the experiment to which the discriminator is applied. For the current implementation of the PSD discriminator, the FOM is 1.56 , or a $56 \%$ increase in the half-life bound. It is interesting to note that achieving an equal increase in sensitivity by scaling the mass of the proposed experiment would imply increasing the mass from $500 \mathrm{~kg}$ to over $1200 \mathrm{~kg}$. Clearly background rejection is, in general, a very cost-effective technology to pursue.

Analysis of the data stream from the Phase 3 instrument will rely on both the traditional techniques of low-background spectroscopy and new segmentation and pulse-shape discrimination methods. Timing information and signals from the active veto shielding are integrated parts of the event stream stored during operation.

An event data model utilizing the ROOT framework (http://root.cern.ch) has been developed for digitized pulses from HPGe detector segments. Subsequent analysis is facilitated using the ability of ROOT to allow flexible access to the part of each event data object required for the particular analysis step. Analysis of the data stream from the Majorana Project Phase-3 experiment will proceed in steps, with each step reducing the size and multiplicity of the data stream. The steps are described below.

\section{- Step 1-Anticoincidence Time Correlation}

The time of each event is analyzed for correlations with events seen in the anticoincidence shield and/or other events seen in the detector array. Events can be rejected based on observed correlations. Previous experience in the IGEX effort has shown the utility of storing timing information for each germanium detector event 
relative to the last active veto signal. This allows optimal veto timing to be developed and various veto time windows to be explored in an off-line analysis.

- Step 2 - Segment Multiplicity Cut

Events showing energy deposition in more than one detector segment in the array are marked as rejected. Monte-Carlo models of detector segmentation have driven the calculated efficacy of this technique, as described in the Phase 3 sensitivity calculation. More complete Monte-Carlo models are underway, and will further refine the conservative estimates of the efficacy of this cut.

\section{- Step 3 -Pulse-Shape Discrimination: Noise Rejection}

Experimental rejection of noise spikes and microphonics has been demonstrated using sophisticated post-processing of each event reaching this phase. Leakage current spikes and high voltage system leakage are two examples of the types of low-rate noise that can be identified. Additionally, analysis of the baseline noise between pulses is a useful stateof-health diagnostic for the detectors of the Phase 3 instrument. Electromagnetic interference, increased leakage current, or thermal fluctuations will become apparent before they have a deleterious effect on detector resolution.

\section{- Step 4-Pulse-Shape Discrimination: Multi-Site Rejection}

Over the past few years, researchers at PNNL and USC have developed and tested an improved class of pulse-shape discrimination techniques. These optimal, self-calibrating, parametric discrimination techniques differ greatly from earlier methods in that they are easily calibrated to individual detector characteristics. These pulse-shape discrimination techniques can be applied to reject multi-site background events, as discussed earlier. This analysis is facilitated using existing codes built with the ROOT object-oriented $\mathrm{C}++$ framework. Additionally, detector segmentation results can be improved by analyzing induced current pulses on all relevant detector segments for consistency with the signature of a single-site interaction.

- Step 5 - Pulse-Shape Discrimination: Event Localization

Experimental data shows that, for single-site interactions, information about the spatial location of the interaction can be extracted from pulse-shape discrimination result, as well as from an analysis of induced currents on all relevant detector segments. Each remaining valid event data object is updated with the best possible information as to the location of the interaction.

- Step 6-Cuts based on inhomogeneities in activity map Single-site events associated with areas of abnormally high activity, for example, due to surface contamination or an isolated "hot spot" in support material, can be rejected via an appropriate small reduction in fiducial volume of the overall detector array. An ongoing map of detector activity is generated as data collection progresses, allowing the identification of any problematic areas. This data can also guide the replacement of any components having higher-than-expected levels of radioactivity.

The rich, multi-parametric nature of the data generated by the Majorana Phase 3 apparatus allows an array of analysis techniques to be applied. This data set will become a resource to which new, more optimal, analysis techniques can be applied as the experiment continues to operate and as new techniques are developed. Additionally, as described in the next section, this data will be available for alternative analysis and the possible extraction of physics results not envisioned at the outset. 


\subsubsection{Proposed Collaboratory Implementation for Majorana}

We propose to facilitate the Majorana collaboration by implementing collaboratory technology at the multiple institutions participating in this research project. The institutions represented in Majorana span the US and Russia. Majorana represents an excellent test bed for collaboratory technology, where we will utilize tools developed at PNNL to provide a collaboratory environment for facilitating DOE High Energy Physics and Nuclear Physics (HEP/NP) division research. The collaboratory implementation could be extended to span the entire underground science laboratory.

Majorana exists as a standalone project, rather than sharing a facility such as an accelerator, providing a project of appropriate scale in isolation from other laboratory infrastructure issues. Majorana also differs from other science projects in that it will be acquiring and analyzing data continuously over a several year period, as distinguished from projects where data is acquired in runs covering days to weeks. A part of this study is to understand how collaboratory tools can be implemented among an existing Majorana scientific collaboration at diverse geographical sites in a non-intrusive way, not interfering with the critical path of completing the Majorana physics project, while the scientists improve their ability to share data, data analysis, notebooks, and critical comments.

This proposed collaboratory implementation aspect of the Majorana project is most naturally directed to the DOE Office of Science's Mathematics, Information, and Computer Sciences (MICS) division.

Collaboratories are designed to encourage closer relationships between scientists in a given research area, to promote collaborations involving scientists in diverse areas, to accelerate the development and dissemination of basic knowledge, and to minimize the time lag between discovery and application. Richard Kouzes originated the PNNL collaboratory effort in 1993, and established this program that continues to be a leading effort for the DOE in collaborative technology development. The Majorana experiment is very different in the science and the interpersonal structure from collaboratories that have been implemented to date. As part of this project, an initial workshop will be held involving the nuclear scientists and the collaboratory technologists to establish the requirements for the Majorana Collaboratory.

A unique opportunity exists to create the collaboratory as an integral part of the experiment design since the Majorana project is just getting under way. The Majorana project would benefit greatly from the introduction of collaboratory tools, such as shared white boards and electronic notebooks, as it moves through the phases of design and construction and into operation and data taking. PNNL developed technology will be tested among the collaborators to determine applicability. This project requires shared analysis and visualization to a greater degree than some of the other collaboratory projects, which will provide a test bed for further developing this technology as a component of the PNNL collaboratory tools. 
Experimenters, technical, and management staff working on Majorana will see several benefits from the use of collaboration technology. One challenge present in Majorana is the large international geographic dispersion of the collaborators, resulting in limited opportunity for real-time interaction, and exacerbated by limitations in communications bandwidth. Among the potential benefits are: 1) daily interaction and access to shared information; 2) faster discovery of problems in experimental data taking; 3) less travel time spent to attend meetings; 4) faster dissemination of results; 5) greater impact of results.

Psycho-social issues of collaboration are the major barrier to success for a collaboratory project. This project will include an evaluation of the sociological factors that might impact on the acceptance of collaboratory technology by the scientific collaborators in order to increase the likelihood of success for this pilot project.

A plan will be developed for the appropriate use of collaboratory tools for Majorana. A workshop will be held bringing together the scientists with collaboratory technologists. A proposal to MICS will be developed for collaboratory implementation for Majorana.

\subsection{Underground Facilities}

The underground facility ultimately selected is pivotal in the design of the Majorana apparatus. However, common features of the key Majorana underground spaces can be identified based on the function of the space. Minor variations on the basic outline presented here will be required to adjust to the specific conditions found in the potential underground location.

\subsubsection{Basic Requirements}

The spaces identified for the Majorana experiment Phase 3 include an experiment hall, an electroforming laboratory, and potentially an underground detector manufacturing laboratory.

The details of the detector manufacturing are considered proprietary by commercial suppliers, but certain parts are well known. Very clean air will be needed in a space the equivalent of 4 meters by 20 meters. Zone refining, crystal pulling, crystal cutting, surface treatment, and testing will be housed in separate rooms within this space. Portions of the process require Class 10 air.

The electroforming process is critical to the project because of the need for lowbackground materials for support structures, vacuum jackets, and so forth. Several plating baths will be required to prepare all the required parts during a production campaign. Fortunately, during production most baths require only periodic monitoring attention.

Electroforming usually takes place in a copper sulphate bath in which a current is passed from a large copper electrode to a mandrel, of appropriate shape. Approximately 20A at $12 \mathrm{~V}$ are required to form a considerable thickness of copper on the mandrel each day. After a few days of copper application, the mandrel and the forming part must be 
removed from the bath and have a thin layer removed to break grain boundaries in the copper and to equalize the growth rate independent of the varying electric fields in the bath. Thus a clean machining room needs to be part of the electroforming facility.

The preparation of the baths requires that the $\mathrm{CuSO}_{4}$ must be recrystallized on occasion to maintain purity. A small hood for this purpose is required. Storage space for raw copper, materials, and parts in progress will be required, as well.

Table 3.5-1 Electroforming Laboratory Requirements

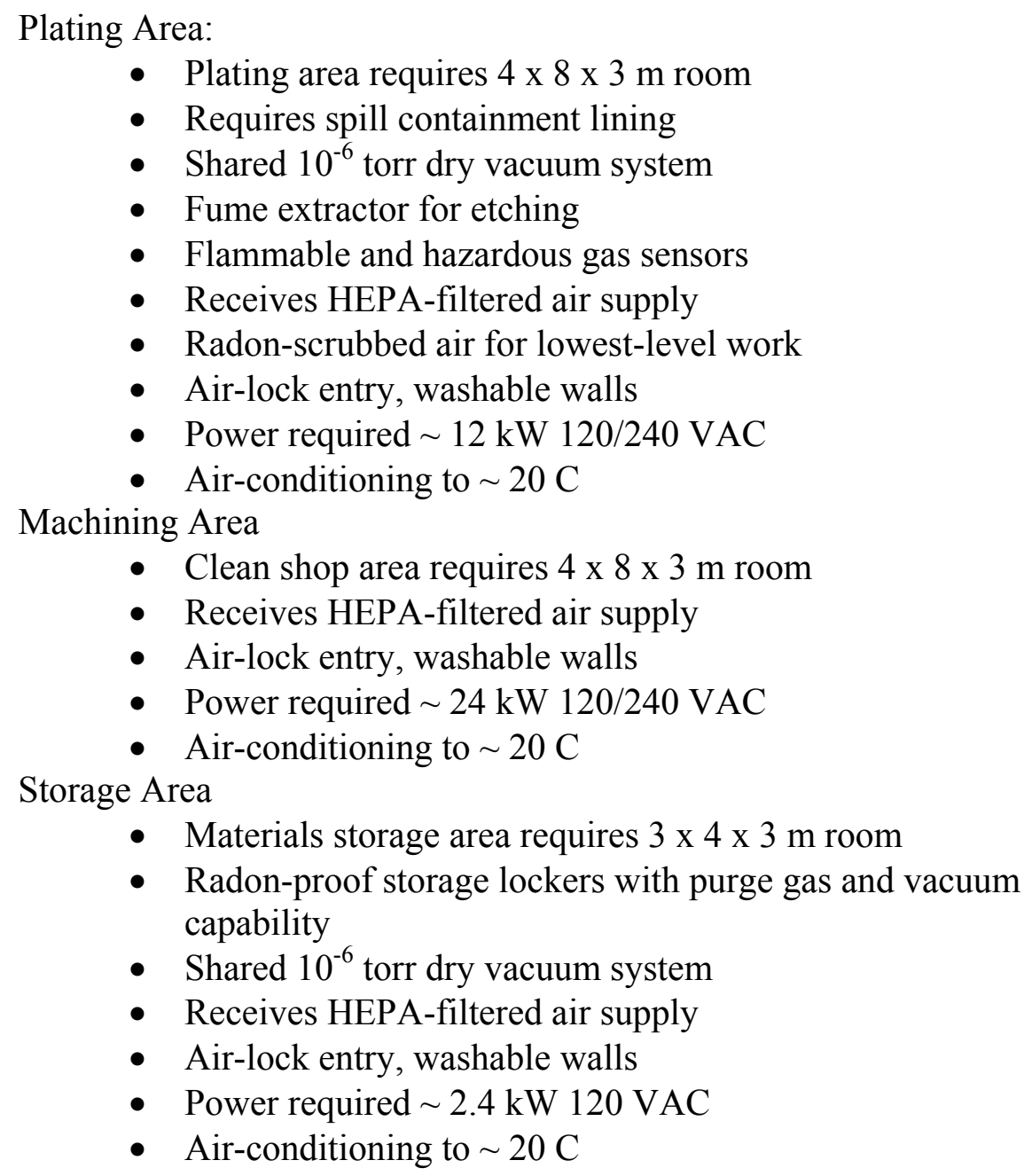

Since the Phase 3 apparatus will consist of ten 21-germanium-crystal modules, periodic installation activity will be required during construction. The periods between these incremental additions will see operation identical to that during the running period after construction is complete, in order to detect problems with the newly installed components, make repairs, and provide feedback into the construction process. The Majorana Phase 3 apparatus will therefore require ease of access and a flexible system providing (1) pre-install staging and work area, (2) expanded space for installation, and (3) a small, isolated space for running. 
The Majorana hall will require an air-locked entry, a control room space, the space for the actual apparatus, and a multi-function anteroom.

Table 3.5-2. Requirements for the Majorana experiment hall.

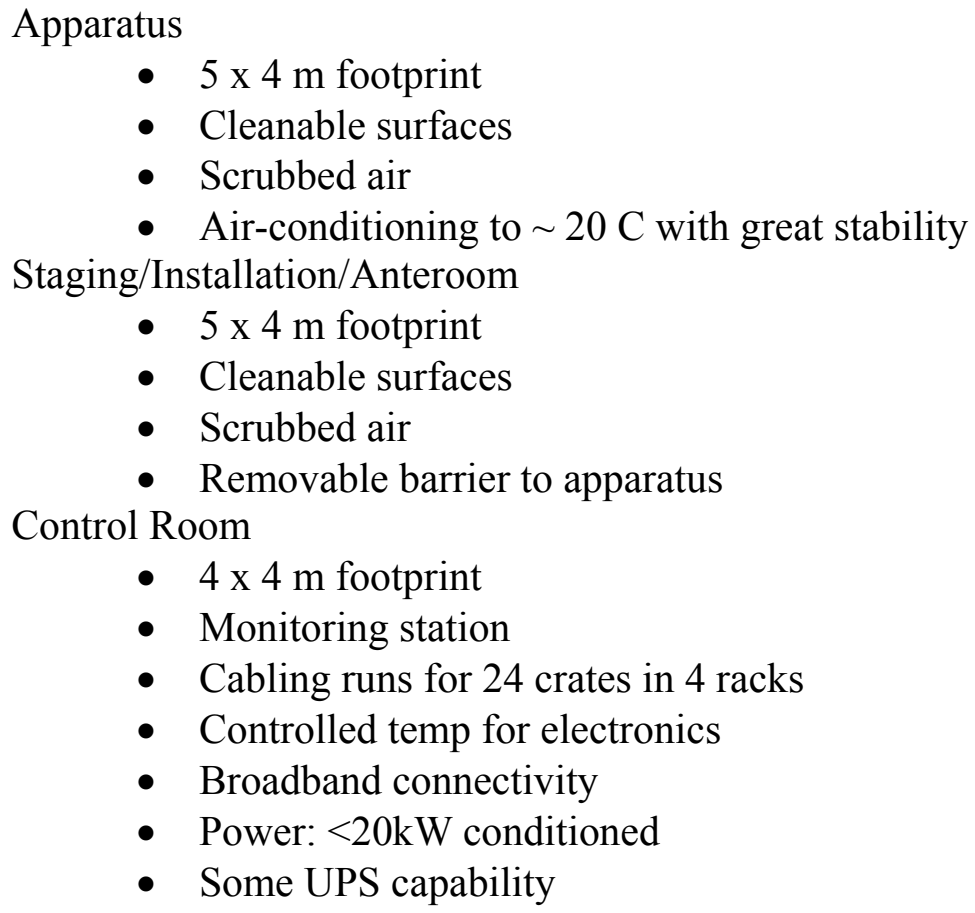

Several approaches are possible in organizing the space needed for Majorana: an organic concept, with all the non-proprietary spaces connected, either in a 'square' or 'linear' arrangement depending on the nature of the underground space, or separate functional facilities, each with airlocks, dressing rooms, and so forth.

\subsubsection{Other Processes}

There are several infrastructure needs which the Majorana project will require. Some are expected to be provided by the laboratory and others are not. The typical facilityprovided infrastructure items include for example power (filtered and unfiltered), provision for exhausting nitrogen gas and filtered hood effluent. Also, broadband network connectivity is required and expected from the facility.

Air conditioning is a typical requirement: it extends the lifetime of electronic and electrical devices and improves the productivity of human workers. In the case of ultralow level experiments, the potassium in sweat contains enough ${ }^{40} \mathrm{~K}$ to spoil a run with a single drop inside the hand-built shield. In addition to normal air conditioning, the temperature of the electronics and the apparatus itself must be maintained with stability to prevent shifts in gain during the period between calibrations. Typical temperature dependence of gain in an ORTEC 572 amplifier is around $10 \mathrm{ppm} /$ degree C. Thus a 10 
degree shift lasting for a substantial time would shift the $2039 \mathrm{keV}$ region by $0.2 \mathrm{keV}$. This is not a large effect, but can be easily prevented.

Other examples of atypical infrastructure include ultra-clean air. In past experiments, the requirement of excluding radon from the spaces around the detectors was achieved by venting nitrogen boil-off gas into the lead cave. This greatly inhibits the inflow of nitrogen into a well sealed lead cave, but does nothing to prevent the deposition of radon daughters in the inner spaces during construction and maintenance. A supply of air which has been scrubbed of radon and subsequently filtered of particulates could eliminate this source of background in the low energy region.

Another experiment-specific type of infrastructure is the provision of liquid nitrogen into the apparatus for cooling. If an adequate supply is provided at the surface, a simple manifold system external to the Majorana experiment hall would be cheap and beneficial.

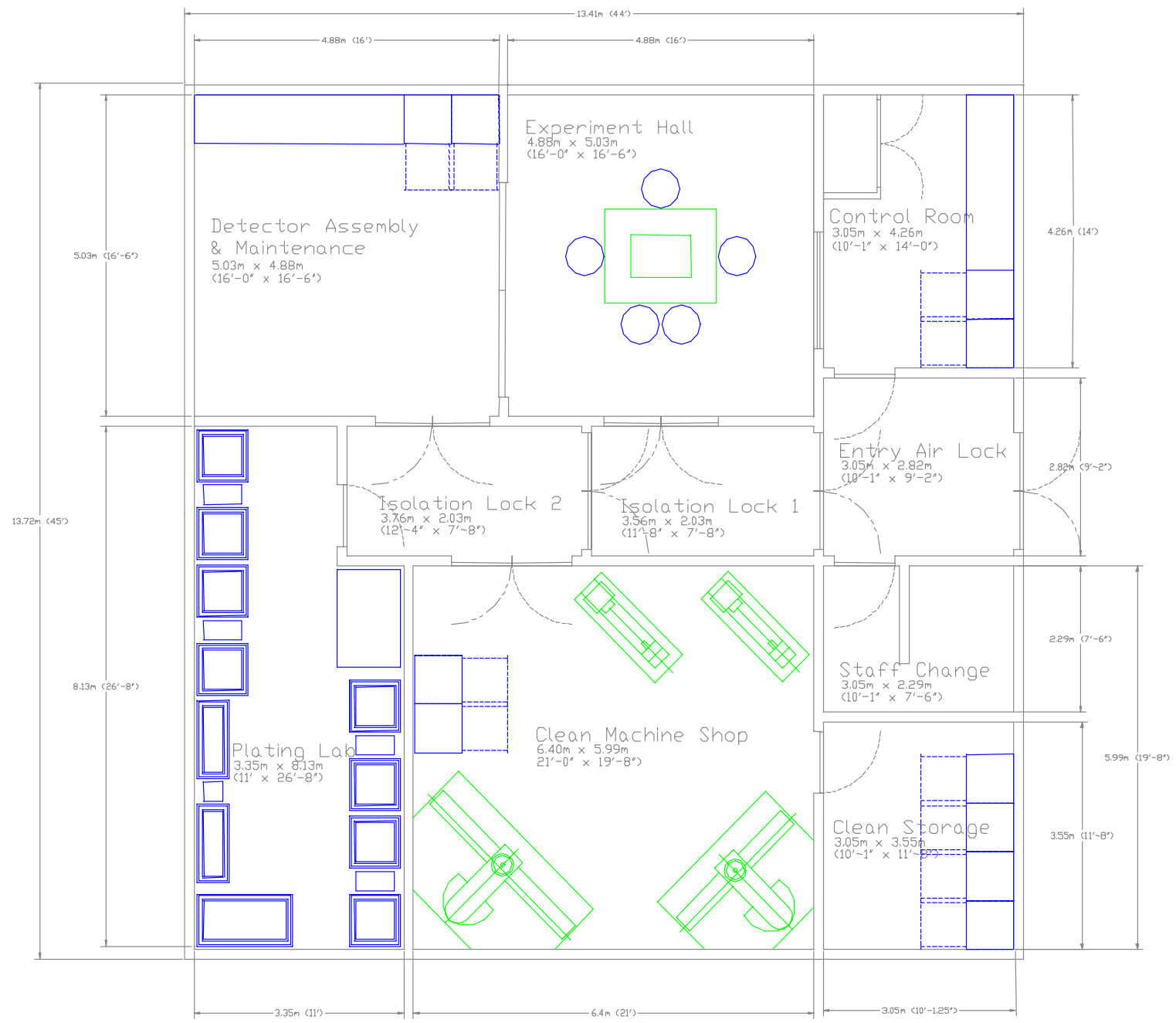

Figure 3-20. Notional layout of Majorana production and experiment spaces. This manifold would allow the introduction of liquid from 160-liter Dewars with an 
absolute minimum of human attention and no entry into the apparatus chamber. This would require careful design to prevent the inadvertent inclusion of radon and moistureladen air.

In the event that no local supply of liquid nitrogen can be obtained, a set of nitrogen distillation systems could provide the supply, requiring only power. The nitrogen could then be introduced through the same manifold system. Adequate space near the Majorana experiment hall would be required for the distillation station.

\subsubsection{WIPP}

The U.S. Department of Energy currently operates the Waste Isolation Pilot Plant (WIPP) near Carlsbad, New Mexico, as a disposal site for transuranic (TRU) waste generated as part of the nuclear defense research and production activities of the federal government. TRU waste is contaminated with alpha-emitting radionuclides that are heavier than uranium (that is, their atomic numbers are greater than that of uranium) and that have half-lives longer than 20 years at concentrations greater than 100 nanocuries $(13,700$ Becquerels) per gram of waste. DOE is responsible for the management and ultimate disposition of TRU waste generated at DOE sites and, as directed by Congress, has constructed WIPP for the purpose of disposing of TRU waste resulting from defense activities. Overall, the WIPP facility is managed by DOE's Office of Environmental Management, which has the principal mission of cleaning up environmental sites at DOE facilities and disposing of radioactive waste. The current Management and Operations Contractor for the WIPP is Westinghouse TRU Solutions.

WIPP is located in Eddy County in southeastern New Mexico approximately 50 kilometers (30 miles) east of Carlsbad, New Mexico. The major construction activities at WIPP have been completed. Surface facilities have been constructed, including the Waste Handling Building where TRU waste is received, inspected, and moved to the waste handling shaft for transfer underground. The constructed underground facilities include four shafts, an experimental area, an equipment and maintenance area, and connecting tunnels. These underground facilities were excavated in the Salado Formation, 655 meters $(2,150$ feet) beneath the land surface. DOE also has excavated the first panel, which consists of seven disposal rooms. This panel currently is receiving waste. A second panel has also been constructed and stands ready for waste emplacement.

DOE now has proposed to expand the availability of WIPP facilities and infrastructure to scientists who wish to conduct experiments there, to the extent such experiments can be conducted without interfering with WIPP's primary TRU waste disposal mission and to the extent that they reflect contemporary budget priorities. The deep geologic repository at WIPP could provide a suitable environment for experiments in many scientific disciplines, including particle astrophysics, waste repository science, mining technology, low radiation dose physics, fissile materials accountability and transparency, and deep geophysics. Currently, one experiment in astrophysics that has been conducted for several years by Los Alamos National Laboratory (LANL) is located in WIPP. Six other 
teams of scientists already have proposed astrophysics experiments to DOE and are seeking funding from the scientific community for those experiments.

Scientists see the WIPP site as having two principal advantages over other facilities throughout the world. First, because WIPP is owned by the U.S. government and its purpose is not to sell resources extracted during excavation, access to WIPP is not likely to be affected by economic demand for the extracted resources as it would in a commercial mining environment. Many such sites are in working, privately owned mines that do not offer the same level of stability, particularly for experiments that may take two decades or more to reach conclusions. Second, because the WIPP site is in the United States, use of the WIPP site would reduce travel and living expense costs for U.S. scientists, many of whom have been traveling to Japan or Italy to conduct their experiments. Allowing the use of the WIPP facilities for these experiments would further the mission of the national scientific community and the DOE Office of Science, and ultimately benefit taxpayers by decreasing the total costs of experimental programs funded by the government.

Because normal background radiation levels can interfere with many experiments, the low background radiation in the WIPP underground facility is one of the factors that makes the site an attractive environment for experiments relating to particle astrophysics, low radiation dose physics, fissile materials accountability, and transparency. Further, WIPP's status as a working underground geologic waste repository also makes it a unique resource for experiments in other fields such as mining, waste repository science, and deep geophysics.

Of particular interest to the current astrophysics and basic science proposals is an area of WIPP once planned for underground experiments. This area was among the first excavated at the WIPP site. Excavations in the area, now known as the North Experimental Area, are as long as 1,384 meters (4,540 feet). They are connected to the disposal area by a series of tunnels, each 10 meters (33 feet) wide and 6 meters (20 feet) high. These tunnels, in turn, are crossed by rooms of about the same size as the tunnels every 100 meters (330 feet). The North Experimental Area is largely unused. It is not a part of the disposal area, and there are no plans to use it for disposal. One hallway and two rooms crossing that hallway have been identified as a potential location for astrophysics and basic science experiments.

\subsubsection{NUSL}

In May 2001, based on the recommendation of the Bahcall Committee and the endorsement of the Nuclear Physics Long Range plan, a proposal was submitted to NSF to convert the Homestake Mine into a National Underground Science Laboratory (NUSL). The proposal calls for 5 year construction plan to provide an underground laboratory at the 7400 foot level along with "campus like" support facilities located on the surface.

The mine has a long scientific history, hosting the pioneering Chlorine Solar Neutrino experiment as well as Ge detectors operated by members of the Majorana Collaboration. 
During the 5 year NUSL construction period it is planned to support and maintain an active scientific program, including the establishment of an ultra low background counting facility and a cosmogenic decay storage facility for materials.

This results in an excellent synergy with the phased plans of the Majorana program.

A $6 \times 6 \times 4 \mathrm{~m}$ high room is needed to accommodate the Majorana Phase- 2 detectors and shield. In addition we require an $11 \times 8 \times 3 \mathrm{~m}$ high room to house the electroforming facility. Ideally we would like these rooms to be contained in the same underground chamber. This chamber should be a long-term structure having level concrete floors, stabilized, shotcreted walls, ventilation and $100 \mathrm{~kW}$ of power. Several potential cavities exist on or near the 7400 foot level proposed for the main laboratory. These cavities, or a new cavity could be made available for the Majorana program.

Phase 3, the full scale Majorana detector, would not be housed in this chamber.

The NUSL plan for developing the underground laboratory calls for customized "built to order" halls. The NUSL and Majorana timescales are such that towards the end of Phase 2, Majorana would be in a position to specify custom chamber requirements and NUSL would be in a position to respond. In return the Majorana Phase 2 detectors and equipment would be used to compliment the Low Background Counting Facility of NUSL, as appropriate.

The local support infrastructure needs of Majorana are expected to be compatible with those anticipated to be available during the 5 year NUSL construction program. Surface facilities would consist of office space for up to 5 persons plus modest materials receiving/storage space. The 7-ton hoist capacity and dimensions are expected to be adequate for materials moving. This would consists of the prefabricated rooms plus the equipment to be installed in them, including $\sim 30$ tons of shielding materials, some of which already exist underground at Homestake. During construction and electroforming operations, daily access for up to 5 persons would be negotiated to integrate with the main NUSL construction activities.

Normal operations would require the delivery of a 160-liter liquid nitrogen Dewar and the occasional removal of liquid waste from the electroforming process. The mine supports state-of-the-art communications and data transmission systems which will be more than adequate for the needs of Majorana. An OC-1 (52 Mbps) direct fiber connection to the outside world is provided by Black Hills Fibercom and will allow remote monitoring and data access. Once Majorana Phase 2 is installed and operating, personnel access requirements are expected to be minimal. 


\subsection{Proposed Majorana R\&D Program (Phases 1 and 2)}

The main physics goal of the Majorana experiment will result from Phase 3 of the plan. However, a short R\&D program is required to optimize and streamline the effort in Phase 3. Several key activities and procurements needed have yet to have resources ascribed to them. These R\&D needs have been categorized into two parts, as described above. Phase 1 , in which segmentation and pulse shape methods are optimized on a few single crystals, and Phase 2, in which many crystals (18) are operated together to establish and demonstrate electronic and mechanical methods for Phase 3.

Table 4-1 R\&D Issues for Majorana Phase 3 Implementation

\begin{tabular}{cl}
\hline Mostly Phase 1 \\
\hline - & Pulse-shape discrimination performance \\
- & Segmentation performance vs. granularity \\
- & Advanced uses of segmentation signals \\
\hline Phase 1 and 2 \\
\hline - & Background models \\
- No-Hit" segment signal analysis methods \\
- Multiple-scatter event tracking and \\
reconstruction for background identification \\
Front-end electronics \\
$\quad$ Radiopurity \\
- $\quad$ Rise-time performance of assembly and testing \\
- $\quad$ Eeasurement of fast neutron background \\
\hline Mostly Phase 2 \\
\hline
\end{tabular}

\subsection{Majorana Phase 1 and 2 Science Goals}

The first two phases of the Majorana project will address the R\&D and testing issues listed in Table 4-1. However, this R\&D program is not without exciting, degreeproducing physics goals as well. These include:

- Double Beta Decay

- $2 v \beta \beta$ : Phase 2 measurement of $2 v \beta \beta$-decay to $0^{+}$excited-state in a number of materials 
- $2 v \beta \beta$ : precision re-measurement of ${ }^{76} \mathrm{Ge}$ decay to the ground state. (Note: the similarity of the $2 \mathrm{n}$ signal to the $0 \mathrm{n}$ signal will be an important test of the electronic signal processing efficacy of the Majorana approach)

- Dark Matter:

- Rapid ( $<1$ year) exclusion of the DAMA CDM result (Phase 1 or 2)

- Solar Axion:

- Phase 2 will be the largest-active-mass Ge solar axion experiment to date

\subsection{Scope of Work}

We propose to continue the Phase 1 program of work by procuring detector materials, producing (through US manufacturers) test detectors and operating them for the purpose of optimizing the background rejection methods we have demonstrated or simulated.

Enriched germanium chemistry differs from that of natural germanium. Therefore we plan to test crystal production at the US detector manufacturers facilities using enriched germanium. We propose to procure approximately $20 \mathrm{Kg}$ to build into Phase 2 detectors. This will test the manufacturers ability to make segmented, n-type detectors with enriched germanium.

The scope for the Phase 2 program is distinct, procuring electronics, building an underground test facility, acquiring data, and creating acquisition code and discrimination code from the methods previously developed at PNNL.

\subsection{Approach}

Phase 1: While we could move forward with our nominal segmentation design presented above (6 azimuthal and $2 \mathrm{z}$ segments), the degree of segmentation is a cost driver for detector production and number of electronic channels. We also wish to experiment with implementing segmentation to the more common p-type detectors, for which there are many more manufacturers. If successful, this could greatly increase the possibility of identifying a manufacturer willing to fabricate detectors at an underground facility. This would result in significant reduction of cosmogenic backgrounds.

Thus, we need to procure two designs each from the two US manufacturers of segmented detectors to optimize the segmentation geometry and also need to experiment with postproduction, unsegmented p-type detectors.

At the trace level, enriched material is chemically quite different from common sources of natural material in the US. If the initial chemical processing of the material could be carried out by Russian instead of US facilities, it would reduce costs and exposure time to cosmic rays. We propose to test this option and the subsequent ability of US manufacturers to construct detectors from this material. Procuring enriched material will constitute a significant part $(>50 \%)$ of the cost of the R\&D proposal for the first fiscal year. Since we wish to test both US manufacturers, we will require $2 \times 10 \mathrm{~kg}$ of enriched material. 
Phase 2: While we are making significant progress in the production of the Phase 2 apparatus using existing resources, we need funds for instrumentation of the resulting system. In addition, we need an underground facility to locate this apparatus.

The Phase 2 instrument will consist of 18 crystals operated together. The inner 2 detectors will be segmented. We have obtained $2070 \%$ relative efficiency ptype detectors to use for this instrument. These may be used for the outer ring of 16 detectors. The annular cryostat and

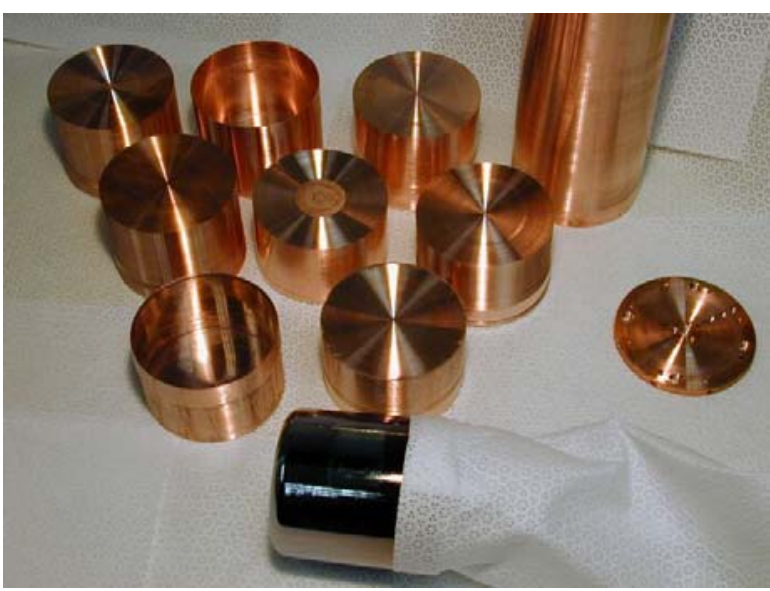

Figure 4-1 The Phase 2 copper is in progress, and detector crystals are in hand. the inner support assemblies needed are currently under construction.

With the inner detectors are segmented at the level of $6 \times 2$, there will be 40 channels of signal processing required. We currently favor a type of acquisition system which is composed of a fast (40 MHz), high bit-depth (13-bit) digitizer with high-resolution event time-stamping. These are 'Fast CAMAC' units and are not common among the collaboration members, such that special procurement will be required at about $\$ 3 \mathrm{k}$ per channel plus supporting control electronics (e.g. crates, controllers etc.). In addition, we are experimenting with commercial preamplifiers which, at a cost of approximately $\$ 500$ per unit may be a cost effective solution.

Finally, the front end of the preamplifier consists of a specially selected and prepared FET with a specially selected resistor and capacitor. In the past, we have manually mounted these in small Teflon pillboxes. Teflon has very high infrared emissivity and thus is generally warmer than other materials in a cryo-jacket. This allows us to achieve the desired FET temperature and also allows the electronics to be mechanically robust against temperature cycling and have low background.

However, the prospect of a thousand such assemblies $(210 \times 6 \times 2)$ would bring the quality assurance of the production process into severe question. Worse, very many vacuum feedthroughs would be needed in the design. To significantly reduce this requirement, we need to explore the possibility of an entire cold preamplifier, with all redundant conductors eliminated in each 21-crystal module.

The underground location for the Phase 2 apparatus will require some special preparation. We wish to create a small, clean facility which will house the Phase 2 apparatus and a control room, and provide clean shielding materials. This will require onsite work in the underground location and will require the procurement and/or shipment of shielding materials. 
Software to acquire the signals is required, as is analysis software for offline reply and categorization of signals of interest. While precursors exist for these two types of software, the majority of the work has yet to be done. 


\subsection{Project Management Plan}

The R\&D Phases of the Majorana experiment will require one or more years to complete and will involve a high emphasis on procurement, with the largest part of the labor coming from faculty/staff/students of DOE nuclear physics labs, US universities, and several Russian institutes. Funding has been and will be applied for by our various collaboration institutions in all appropriate venues.

We envision the coordination of tasks and responsibility for communication at PNNL, with work at home institutions, PNNL labs, and underground locations to be governed by the overall plan of work and the ES\&H policy and training of both the host institution and the home institution of the workers.

Work Breakdown Structure (Completion is indicated with $\square$ )

Phase 1 Detector Procurement

Obtain enriched material

Contract manufacturer

Procure copper cryostat

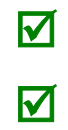

Assembly and test

Process Ge back to oxide / Return $1 / 2 \square$

Repeat for varied segmentation designs

Phase 1 and 2 Acquisition Electronics Procurement

Procure HV

$\square$

Procure 40 MHz, 12-bit DAC system (32 channels)

Procure crates, controllers

Phase 1 and 2 Software Development

Define common data format for Sim and Expt

GEANT (etc) simulation of ME design $1 / 2 \square$

Low level drivers for DAC

Control code for acquisition (high level)

Data archival database / web interface

Pulse cutting algorithm

Segmentation cutting algorithm

Event visualization tool

Histogramming tool 


\section{WBS Continued}

Phase 2 Preamplifier Design, and Procurement

Identify source for front-end fabrication (MCM)

Screen component materials for radiopurity

Procure front-end and preamplifier prototypes

Performance thermal and electrical testing of prototypes

Finalize design from prototypes

Procure final preamp and front-end solution

Acceptance thermal and electrical testing of final parts

Phase 2 Detector Procurement

Obtain Ge crystals for outer toroid

Procure copper toroid and related

Obtain inner Ge crystals

Test segmentation scheme

Procure copper for inner detectors

Assembly and test

Phase 2 Underground Preparations

Collection of low bkg Pb, CPUs, electronics, veto shielding $\quad 1 / 2$ च

Movement of materials to selected location

Preparation / cleaning of enclosure underground

Shielding assembly

Detector Installations

Phase 1 and 2 Testing and Operation

Phase 1 shakedown testing

Phase 1 experiment run

Phase 2 shakedown testing

Phase 2 experiment run

Enriched Materials Acquisition

Exploratory meeting

Contracting / negotiation meeting

Materials receipt and testing 


\subsection{Project Schedule}

The Majorana experiment will be implemented in 3 phases as discussed earlier. The schedule for the 3 phases overlap, and work on Phases 1 and 2 has already begun. The following projects the schedule for each of the Majorana experiment phases.

The phases have been defined along the main technical lines of the overall project.

- Phase 1: Development of signal processing techniques for segmented crystals.

- Phase 2: Develop packaging for multiple crystals sharing a single cooling system.

- Phase 3: Including all the above plus a large quantity of enriched materials.

Several features have not been explicitly discussed. First, detectors produced in Phase 1 may be introduced into the Phase 2 apparatus for joint testing, as appropriate. Second, that Phase 1 detectors could be produced from enriched material if Phase 3 gets an early start. This does not further the R\&D goal of Phase 1, but would give us a better potential for short-term physics results from Phase 1.

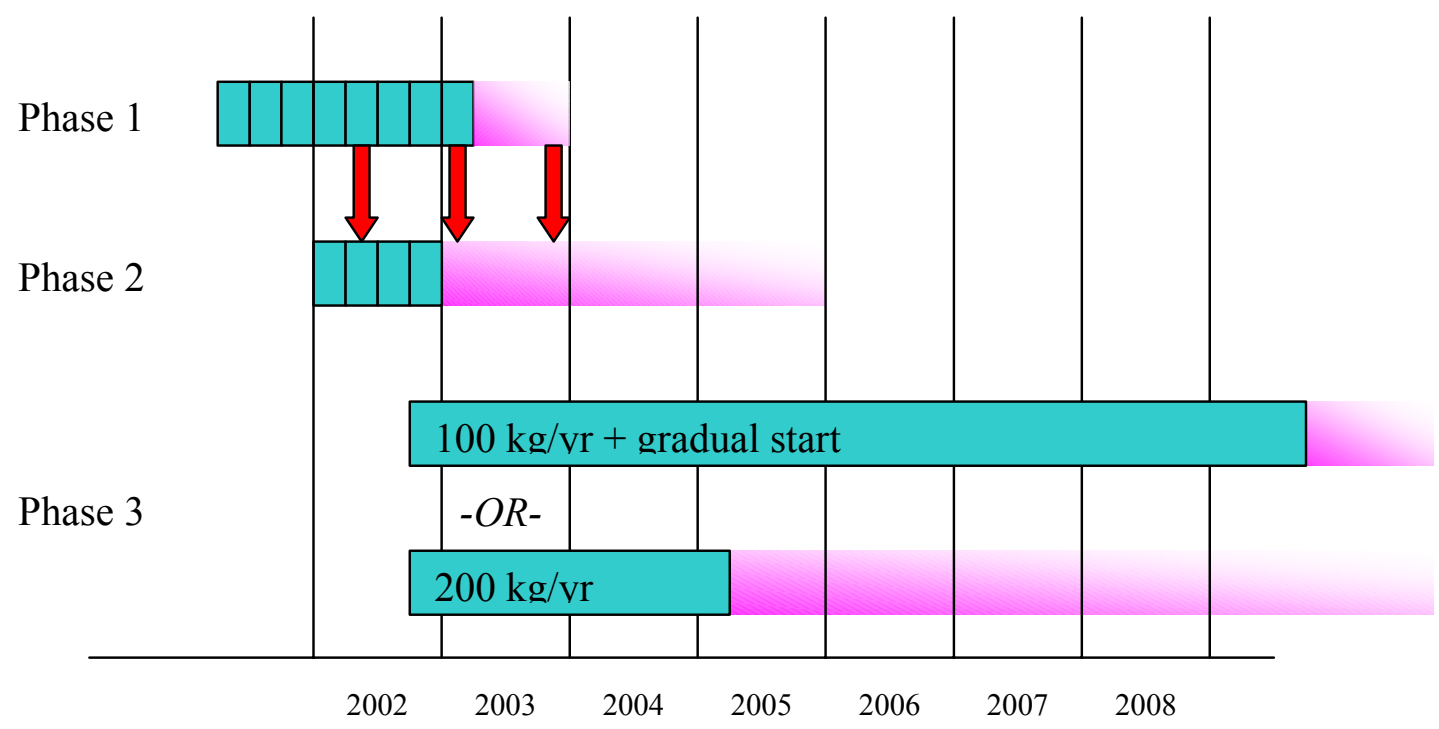

Figure 6-1 Nominal project schedule for the Majorana baseline plan. Construction periods are shown in blue, data acquisition in pink. Not shown is the complete running period for Phase 3. The beneficial effect of acquiring data during construction is shown in section 2.3 above. Shown are two options for enrichment, first a gradual start to enrichment ramping to $100 \mathrm{~kg} / \mathrm{yr}$ and second immediate production of $200 \mathrm{~kg} / \mathrm{yr}$. Enrichment rates higher than $200 \mathrm{~kg} / \mathrm{yr}$ may be possible, but have not yet been discussed with providers. 
There will be important physics results arising from each phase of the experiment. The ultimate running period for the full experiment is planned as ten years of operation. The following milestones are based upon a 2002 start for funding of Majorana R\&D Phases $1 \& 2$, followed a year later by full funding for Phase 3 .

Milestone 1:

Milestone 2:

Milestone 3:

Milestone 4:

Milestone 5:
First Phase 1 Detector Operational

Phase 2 Operational

Phase 1 First Axion results

Phase 2 First Dark Matter results

Phase 1 First $2 v \beta \beta$ results
August 2002
December 2002
Summer 2003
Summer 2003
Summer 2003

Assume slow baseline (i.e. gradual ramp to $100 \mathrm{~kg} / \mathrm{yr}$ )
Milestone 6s:
Phase 3 reaches $T_{1 / 2}=2.510^{26}$
Milestone 7s:
Phase 3 reaches $T_{1 / 2}=1.010^{27}$
Phase 3 mass complete at $500 \mathrm{~kg}$
Milestone 8s:
Phase 3 reaches $T_{1 / 2}=3.810^{27}$
Spring 2005
Spring 2009
Spring 2009
Spring 2019

Assume fast baseline (i.e. immediate $200 \mathrm{~kg} / \mathrm{yr}$ )

Milestone 6f: $\quad$ Phase 3 reaches $T_{1 / 2}=2.510^{26}$

Spring 2003

Milestone 7f:

Phase 3 mass complete at $500 \mathrm{~kg}$

Spring 2005

Milestone 8f:

Phase 3 reaches $\mathrm{T}_{1 / 2}=1.010^{27}$

Milestone 9f:

Phase 3 reaches $T_{1 / 2}=3.810^{27}$

Fall 2004

Summer 2013

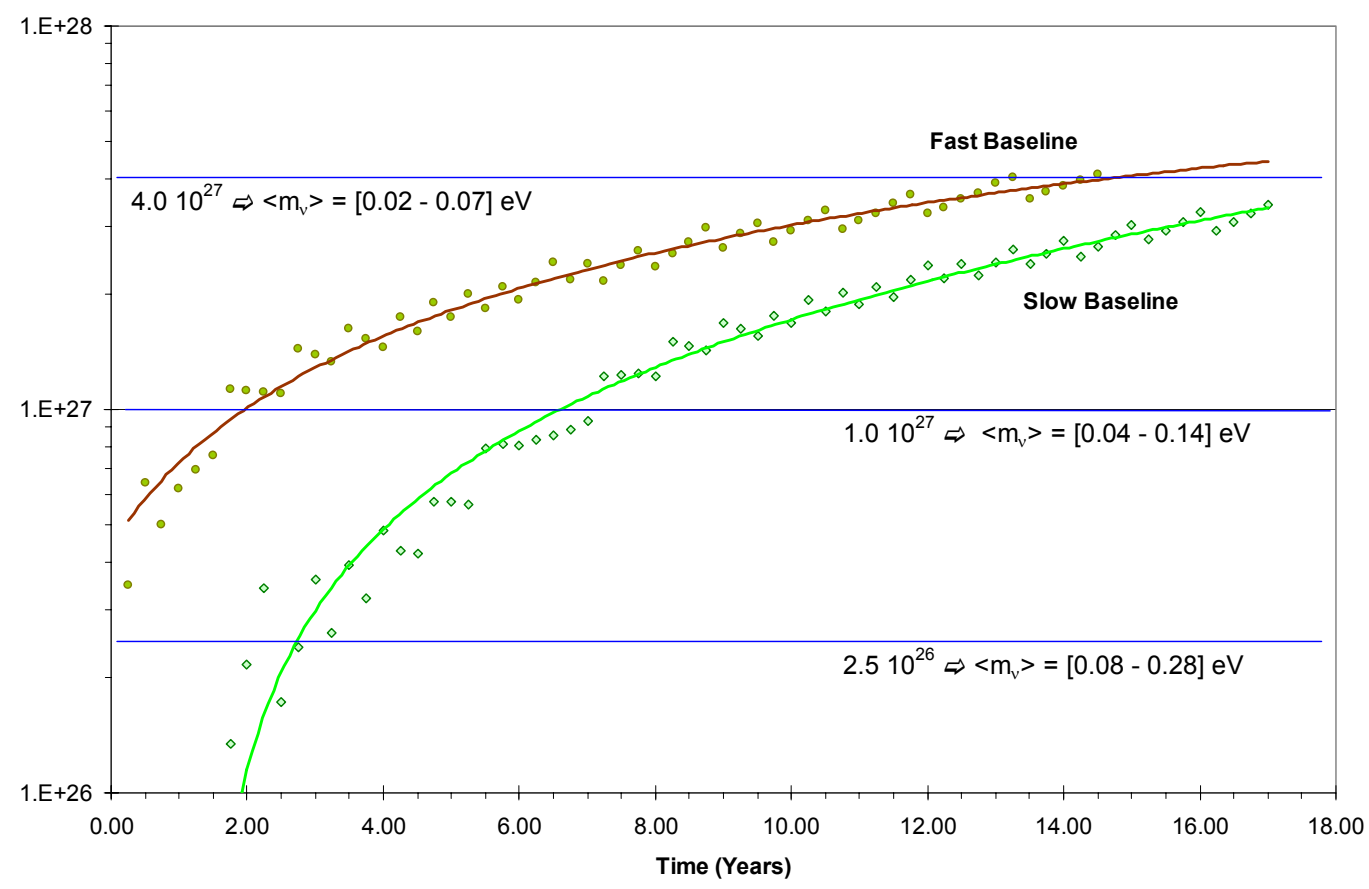

Figure 6-2 Comparison of fast and slow baseline sensitivity growth assuming data acquisition during construction. Both of these scenarios have been constructed to minimize cost. The fast baseline could attain the final result $\left(T_{1 / 2}=\sim 3.810^{27}\right.$ and $\left.<m_{v}>=[0.02-0.07] \mathrm{eV}\right)$ in as little as 13.5 years. 


\subsection{References}

[Aa199a] C. E. Aalseth, R. J. Arthur, and J. H. Reeves. In 1999 IEEE Nuclear Science Symposium. Conference Record, 2 (1999) 378-381

[Aa199b] C. E. Aalseth, and H. S. Miley. In 1999 IEEE Nuclear Science Symposium. Conference Record, 2 (1999) 769-771

[Abu00] R. Abusaidi et al., Phys. Rev. Lett. 84 (2000) 5699

[Ahm01] Q. R. Ahmad, et al., (the SNO Collaboration), Preprint submitted to Phys. Rev. Lett. (17 June 2001).

[Art93] V. A. Artem'ev, E. V. Brakhman, O. Ya. Zel'dovich, A. K. Karelin, V. V. Kirichenko, O. M. Kozodaeva, V. A. Lyubimov, A. I. Mitin, V. N. Paramokhin, T. N. Tsvetkova, S. I. Vasil'ev, A. A. Klimenko, S. B. Osetrov, A. A. Pomanskii, and A. A. Smol'nikov, JETP Lett. 58 (1993) 262

[Avi00] F. T. Avignone, C. E. Aalseth, and R. L. Brodzinski, Phys. Rev. Letts. 85 (2000) 465

[Avi01] F.T. Avignone et al., Proceedings of Taup-2001, 8-12 September 2001, L'Aquila, Italy (in press).

[Avi88] F. T. Avignone and R. L. Brodzinski, J. Prog. Part. Nucl. Phys. 21 (1988) 99

[Avi91] F. T. Avignone et al., Phys. Lett. B256 (1991) 559

[Avi92] F. T. Avignone et al, Nucl. Phys. B (Proc. Suppl.) 28A (1992) 280-285.

[Avi98] F.T. Avignone III et al., Phys. Rev. Lett. 81 (1998) 5068

[Avi99] F.T. Avignone III et al., Nucl. Phys. B (Proc. Suppl.) 72 (1999) 176

[Ba101] E. A. Baltz, P. Gondolo, Phys. Rev. Lett. 86 (2001) 5004

[Bar95] A. S. Barabash, F. T. Avignone III, J. I. Collar, C. K. Guerard, R. J. Arthur, R. L. Brodzinski, H. S. Miley, J. H. Reeves, J. R. Meier, K. Ruddick, and V. I. Umatov, Phys. Letts. B345 (1995) 408

[Bat01] M. Battaglia et al., arXiv: hep-ph/0106204

[Bau99] L. Baudis, et al., Phys. Rev. Lett. 83 (1999) 41

[Bau00] L. Baudis et al., arXiv: hep-ex/0012022.

[Bed97] V. Bednyakov arXiv: hep-ph/0201046; (see also V. Bednyakov et al., Z.Phys.A 357 (1997) 339

[Bel89] P. Belli et al., Il Nuovo Cimento 101 (1989) 959; S.R. Hashemi-Nezhad et al., Nucl. Instr. Meth. A357 (1995) 524; J.N. Abdurashitov et al., arXiv: physics/0001007.

[Ber01] L. Bergstrom, Rep. Prog. Phys. 63793 (2000); M. S. Turner, (2001), arXiv: astro-ph/0104460 
[Bil01a] S. M. Bilenky, S. Pascoli and S. T. Petcov, arXiv: hep-ph/0105144 v1 15 (May 2001)

[Bil01b] S. M. Bilenky, S. Pascoli and S. T. Petcov, Phys. Rev. D64 (2001) 053010

[Bi199] S. M. Bilenky, C. Guinti, W. Grimus, B. Kayser, and S. T. Petcov, arXiv: hep-ph / 9907234 v3, 30 August 1999; Phys. Lett. B465 (1999) 193

[Bot00] A. Bottino, F. Donato, N. Fornengo, and S. Scopel, arXiv: hep-ph/0010203; see also Phys.Rev. D62 (2000) 056006

[Bro90] R. L. Brodzinski et al, NIM A292 (1990) 337-342

[Bro95] R. L. Brodzinski, et al, Journal of Radioanalytical and Nuclear Chemistry, Articles, Vol. 193, No. 1 (1995) 61-70.

[Ceb01] S. Cebrian et al., Astropart. Phys. 14, (2001) 339 arXiv: hep-ph/9912394; F. Hasenbalg, Astropart. Phys. 9 (1998) 339; arXiv: astro-ph/9806198.

[Col00] J. I. Collar et al., New Journal of Physics 2 (2000) 14.1 (http://www.njp.org).

[Co192] J.I. Collar, Ph. D. dissertation (unpublished), University of South Carolina, 1992.

[Co199] J. I. Collar, Phys. Rev. D59, (1999) 063514; arXiv: astro-ph/9808058.

[Cor00] A Corsetti and P Nath, arXiv: hep-ph/0003186

[Cre98] R. J. Creswick et al., Phys. Lett. B427 (1998) 235

[Dru86] A. K. Drukier et al., Phys. Rev. D33 (1986) 3495

[Dru92] A. K. Drukier et al., Nucl. Phys. B (Proc. Supp.) 28A (1992) 293.

[Eds97] J. Edsjo and P. Gondolo, Phys. Rev. D56 (1997) 1879

[Eji91] H. Ejiri, K. Fushimi, M. Kawasaki, H. Kinoshita, H. Ohsumi, K. Okada, H. Sano, T. Shima, E. Takasugi, J. Tanaka, and T. Watanabe, J. Phys. G17, (1991) S155

[El100] J. Ellis, T. Falk, G. Ganis, and K. A. Olive, Phys. Rev. D62 (2000) 075010

[El101a] J. Ellis, A. Ferstl, and K. A. Olive “Theoretical Aspects of Dark Matter Detection” (2001) arXiv: hep-ph/0106148

[Ell01b] J. Ellis, A. Ferstl, and K. A. Olive, Phys.Rev. D63 (2001) 065016, arXiv: hep-ph/0007113

[El187] S. R. Elliott, A. A. Hahn, and M. K. Moe, Phys. Rev. Lett. 59 (1987) 2020

[El191] S. R. Elliott, M. K. Moe, M. A. Nelson, and M. A. Vient, J. Phys. G17 (1991) S145

[El193] S. R. Elliott, M. K. Moe, M. A. Nelson, and M. A. Vient, Nucl Phys. B (Proc. Suppl.) 31 (1993) 68

[El197] J. Ellis, T. Falk, K. A. Olive, and M. Schmitt, Phys. Lett. B413 (1997) 355 
[Far01] Y. Farzan, O. L. G. Peres, and A. Yu. Smirnov, Preprint, arXiv: hep$\mathrm{Ph} / 0105105$ v3, 26 May 2001

[Fio67] E. Fiorini, A. Pullia, G. Bertolini, F. Cappellani, and G. Restelli, Phys. Lett.25B (1967) 602

[Fir48] E. L. Fireman, Phys. Rev. 74 (1948) 1238

[Fre92] K. Freese et al., Phys. Rev. D37 (1988) 3388

[Gai01] R.J. Gaitskell, 3rd Intl. Workshop on Identification of Dark Matter (World Scientific, September 2001) arXiv: astro-ph/0106200.

[Goo85] M. W. Goodman and E. Witten, Phys. Rev. D31 (1985) 3059

[Hes59] W. N. Hess et al, Phys. Rev. 116 (1959) 449

[Ing50] M. G. Inghram and J. H. Reynolds, Phys. Rev. 78 (1950) 822

[Ira00] I.G. Irastorza et al., Nucl. Phys. (Proc.Suppl.) 87, (2000) 102-104 arXiv: astro-ph/9912491

[Jun96] G. Jungman, M. Kamionkowski, and K. Griest, Phys. Rep. 267, (1996) 195

[Kir67a] T. Kirsten, et al., Z. Naturforsch. A22 (1967) 1783

[Kir67b] T. Kirsten, W. Gentner, and O. A. Schaeffer, Z. Phys. 202 (1967) 273

[Kla98] H. V. Klapdor- Kleingrothaus et al, Jour. Phys. G Nucl. Part. Phys. 24 (1998) 483; H. V. Klapdor-Kleingrothaus, Nucl. Phys. B (Proc. Suppl.) (2001) 350

[Kla01a] H. V. Klapdor-Kleingrothaus, H. Päs, A. Yu. Smirnov, Phys. Rev. D63 (2001) 073005

[Kla01b] H. V. Klapdor-Kleingrothaus, et. al., Eur.Phys.J. A12 (2001) 147

[Ko190] E. W. Kolb and M. S. Turner, The Early Universe (Addison-Wesley, Reading, MA, 1990)

[Kud01] V. A. Kudryavtsev et al., arXiv: hep-ex/0109013

[La167] D. Lal, et al, Cosmic Ray Produced Activity on the Earth (Springer, Berlin/Heidelberg, 1967)

[Lee77] B. W. Lee and S. W. Weinberg, Phys. Rev. Lett. 39 (1977) 165

[Lew96] J. D. Lewin and P. F. Smith, Astropart. Phys 6 (1996) 87

[Man00] V. Mandic, A. Pierce, P. Gondolo and H. Murayama, hep-ph/0008022

[Mil90] H. S. Miley, F. T. Avignone III, R. L. Brodzinski, J. I. Collar, and J. H. Reeves, Phys. Rev. Lett 65 (1990) 3092

[Mil94] H. S. Miley, et al, Nucl. Phys. B (Proc. Suppl.) 35 (1994) 388

[Pas01] S. Pascoli, S. T. Petcov, and L. Wolfenstein, Preprint, arXiv: hep-ph/0110287 v2, 6 Dec 2001. 
[Pec77] R.D. Peccei and H.R. Quinn, Phys. Rev. Lett. 38 (1977) 1440; Phys. Rev. D16 (1977) 1791

[Pec89] R.D. Peccei, in "CP Violation”, ed. C. Jarlskog (World Scientific, Singapore, 1989).

[Pee93] P. J. E. Peebles, Principles of Physical Cosmology (Princeton University Press, Princeton, NJ, 1993).

[Pri88] J. R. Primack, D. Seckel, and B. Sadoulet, Annu. Rev. Nucl. Part. Sci. 38 (1988) 751

[Pry01] C. Pryke et al., arXiv: astro-ph/0104490, submitted to Ap. J; C.B. Netterfield et al., arXiv: astro-ph/0104460.

[Raf96] G. Raffelt in "Stars as Laboratories for Fundamental Physics", (University of Chicago Press, Chicago \& London, 1996.)

[Ros01] L.J. Rosenberg and K.A. van Bibber, Phys. Rep. 325 (2001) 1

[Sa197] P. Salucci and M. Persic, ASP Conf. Ser. 117: Dark and Visible Matter in Galaxies and Cosmological Implications (Brigham Young University, Provo, UT, 1997); 1-27.

[Sik83] P.Sikivie, Phys. Rev. Lett. 51 (1983) 1415

[Sob01] H. Sobel (for the Super-Kamiokande Collaboration), Nucl. Phys. B (Proc. Suppl.) 91, (2001) 127; Y. Fakuda, et al., Phys. Rev. Lett. 81, 1562 (1998); 82, (1999). 1810

[Sre00] Srednicki M, Eur. J. Phys. C15 (2000) 143

[Sri73] B. Srinivasan, E. C. Alexander, Jr., R. D. Beaty, D. Sinclair, and O. K, Manuel, Econ. Geol. 68 (1973). 252

[Ste01] P. Di Stefano et al., Astropart. Phys. 14 (2001) 329; arXiv: astro$\mathrm{ph} / 0004308$.

[Tak66] N. Takaoka and K. Ogata, Z. Naturforsch. A21 (1966) 84

[Vas90] A. A. Vasenko, I. V. Kirpichnikov, V. A. Kuznetsov, A. S. Starostin, A. G. Djanyan, V. S. Pogosov, S. P. Shachysisyan, and A. G. Tamanyan, Mod. Phys. Lett. A5 (1990) 1299

[Vet00] K. Vetter, A. Kuhn, I.Y. Lee, R.M. Clark, M. Cromaz, M.A. Deleplanque, R.M. Diamond, P. Fallon, G.J. Lane, A.O. Macchiavelli, M.R. Maier, F.S. Stephens, C.E. Svensson, H. Yaver, Nuclear Instruments and Methods in Physics Research A452 (2000) 105-114

[Wei78] Steven Weinberg, Phys. Rev. Lett. 40 (1978) 223

[Wil78] F. Wilczek, Phys. Rev.Lett. 40 (1978) 279

[Yar81] Y. Yariv and Z. Fraenkel, Phys. Rev. C24 (1981) 488

[Zio99] K. Zioutas et al, Nucl. Instr. Meth. A425 (1999) 480 


\subsection{Appendices}

\section{Appendix 1. Enrichment Documents}

This appendix consist of two documents: first a memo to interested parties reporting the contents of a meeting hosted by ITEP concerning availability of enrichment services in Russia, second, the text of a Memorandum of Understanding produced at this meeting.

Memo to Agencies:

\begin{tabular}{|c|c|}
\hline & November 9, 2001 \\
\hline \multirow[t]{2}{*}{ Memo to: } & $\begin{array}{l}\text { Interested Officials at the Office of Science of the U. S. DOE and the } \\
\text { Physics Division of the National Science Foundation }\end{array}$ \\
\hline & $\begin{array}{l}\text { Harry S. Miley, Pacific Northwest National Laboratory } \\
\text { Frank T. Avignone, III, University of South Carolina } \\
\text { Majorana Project Spokesmen }\end{array}$ \\
\hline Background: & $\begin{array}{l}\text { The } 2001 \text { NSAC Process placed high priority on the development of a } \\
\text { National Underground Laboratory and on the appropriate physics to be } \\
\text { performed there. A priority is implied on an American-based, next-generation } \\
\text { neutrinoless double-beta decay experiment. The Majorana collaboration } \\
\text { submits that a next-generation }{ }^{76} \mathrm{Ge} \text { experiment is one most ready for } \\
\text { deployment. A number of questions have been raised concerning the costs, } \\
\text { quantities and delivery schedule possible from the appropriate Russian } \\
\text { institutes of high quality germanium, isotopically enriched to } 85 \% \text { in }{ }^{76} \mathrm{Ge} \text {. } \\
\text { This memo is intended to address some of those questions. }\end{array}$ \\
\hline \multicolumn{2}{|c|}{$\begin{array}{l}\text { On October 29, 2001, Majorana scientists (Harry Miley, Frank Avignone, and } \\
\text { Alexander Barabash), attended a meeting with representatives of the Electrochemical Plant } \\
\text { (ECP), Zelenogorsk in Krasnoyarsky, Krai, Russia (previously referred to as K-45). The } \\
\text { meeting was held at the Institute for Theoretical and Experimental Physics (ITEP), Moscow, } \\
\text { and was hosted by professor Michael Danilov, Scientific Director of ITEP, which is a founding } \\
\text { member of the Majorana collaboration. Present were were: Michael Danilov, Vladimir } \\
\text { Zakopaev, Head of the ECP Laboratory, and Elena Nikitina, Manager, ECP Marketing } \\
\text { Department. The meeting was called with far less than } 45 \text { days notice, making the } \\
\text { involvement of federal agency personnel impractical. An outline of answers to key questions } \\
\text { appears in the bullets below: }\end{array}$} \\
\hline \multicolumn{2}{|c|}{$\begin{array}{l}\text { - The Majorana experiment requires } 500 \mathrm{~kg} \text { of radio pure Ge isotopically enriched to } \\
85 \% \text { in }{ }^{76} \mathrm{Ge} \text {. } \\
\text { - The ECP "Department of Superclean Materials" has a track record in providing the } \\
\text { material used in both recent }{ }^{76} \mathrm{Ge} \text { double beta decay experiments. No trace of natural } \\
\text { or other radioactive material has been observed after many kg-years of operation. No } \\
\text { uranium is or has ever been separated in this ECP department. } \\
\text { - The ECP provided a quote of } \$ 56,000 \text { per kilogram for production of up to } 30 \mathrm{~kg} / \mathrm{year} \text {. } \\
\text { For an additional } \$ 50,000 \text {, they would expand their production } 50 \mathrm{~kg} / \mathrm{year} \text {. } \\
\text { - For an estimated cost of } \$ 3.5 \text { million, they would upgrade from } 50 \mathrm{~kg} / \mathrm{year} \text { to } 100 \\
\mathrm{~kg} / \mathrm{year} \text {, and for an estimated cost of } \$ 5.0 \text { million, they would upgrade from } 50 \mathrm{~kg} / \mathrm{year} \\
\text { to } 200 \mathrm{~kg} / \mathrm{year} \text {. }\end{array}$} \\
\hline & Memo to Agencies Page 1 \\
\hline
\end{tabular}


- In years where production increases are funded on October $1^{\text {st }}$, the full upgraded quantity can be delivered that fiscal year.

- The estimated cost of quantities of $100 \mathrm{~kg}$ to $200 \mathrm{~kg}$ would be $\$ 45,000 / \mathrm{kg}$.

- ECP will guarantee the purity of the material to be the same national production standard which was that well tested in both multi-kilogram ${ }^{76} \mathrm{Ge}$ experiments over the last eight years.

- There are no volume limitations that would cause interference between isotopic enrichment of large quantities of ${ }^{76} \mathrm{Ge}$ and other isotopes, ${ }^{136} \mathrm{Xe}$ for example. These facilities are different.

A memorandum of understanding was signed between the Majorana co-spokesmen, the ECP officials, and the ITEP representatives. It does not commit the U. S. side to anything, but it does commit Zelenogorsk ECP to a fixed price for small quantities, and it guarantees the quality to be that which was well tested by IGEX and by Heidelberg-Moscow.

Misha Danilov should be well known to both the NSF and DOE since he and ITEP have collaborated for many years with many American groups, and he is a member of the committee on Russian and American scientific cooperation. His institute has placed a high priority on the Majorana project, and we are convinced that his direct involvement will play a crucial role in obtaining the promised product on the promised schedule from the ECP. In the view of Dr. Danilov, it would be prudent to begin production on some scale to assure the continued interest of the ECP.

The Majorana collaboration plans to submit multi-institutional proposals for a total of a few million dollars to order $30 \mathrm{~kg}$ of isotopically enriched $\mathrm{Ge}$ to complete the remaining tests of our $R \& D$ and to build a substantial demonstration test module that would become part of the Majorana suite of detectors. The first step would be large enough to make an impact on furthering the sensitivity of the Majorana neutrino mass and in making a real impact on the search for Cold Dark Matter. It would demonstrate the key Majorana technology and prove feasibility.

A copy of the memorandum of understanding is attached. The Majorana co-spokesman will request meetings with the appropriate DOE and NSF personnel for detailed discussions. A joint meeting with both agencies would in our opinion be the most effective. We would like to include a number of key players in the collaboration in this meeting.

We want to present our case for the Majorana experiment to the SAGENAP committee at the earliest possible time. We are prepared. This is an exciting opportunity and should not be overlooked or delayed. 
ECP MOU:

\section{Memorandum of Agreement between ITEP, the ECP and the Majorana Collaboration: Enrichment Costs, Schedules, and Technical Issues}

October 29, 2001

\section{Introduction}

The Majorana experiment requires $500 \mathrm{~kg}$ of germanium enriched to $85 \%$ in Ge-76. This material is only available in this quantity from enrichment facilities such as the Electrochemical Plant of Zelenogorsk, formerly known as K-45. Only the ECP Department of Super Clean Materials has a track record in providing germanium which has been operated as ultra-low background detectors for approaching a decade. ITEP and ECP have a long term record of successful collaboration. The purpose of this document is to set out the main features of a discussion held on Oct 29, 2001 at ITEP, Moscow, Russia, on the subject of costs and technical features of a Majorana enrichment campaign. The Majorana collaboration charter identifying collaboration institutions and collaboration goals can be found at majorana.pnl.gov.

\section{Common Specifications}

It was agreed that the special standards used to produce the germanium used in previous germanium double-beta decay experiments were adequate. ECP agreed to guarantee this level of quality, which includes that the material will be at least $85 \%$ Ge-76. ECP has agreed to store all enriched material while not in process in a location with a 20 meter water equivalent overburden to minimize the cosmogenic creation of Ge-68. All local taxes and other costs are included in the quotes and estimates in this MOU. If transport by air is selected by the Majorana collaboration, quotes include shipment to a US port of entry from Zelenogorsk.

\section{Costs and Schedules for Production in Fiscal Year 2002-2003}

The ECP presented firm quotes of $\$ 56 \mathrm{k} / \mathrm{kg}$ for production of germanium in quantity up to $30 \mathrm{~kg} / \mathrm{year}$, and a firm quote for $\$ 50 \mathrm{k}$ for the increase of the capacity from 30 $\mathrm{kg} /$ year to $50 \mathrm{~kg} / \mathrm{year}$. In all cases, the ECP requires $20 \%$ of the purchase cost of the material as a start up to procure raw materials. These quotes will be valid for proposals made by the Majorana collaboration between December 2001 and February 2002 for production up to September 2003. After capacity increase, the enriched material will cost $\$ 56 \mathrm{k} / \mathrm{kg}$.

It is the intent of the Majorana collaboration to produce a proposal for mid year funding which will be submitted in Dec 2001. If this is not funded, a regular schedule proposal will include costs for between 20 and $30 \mathrm{~kg}$ of enriched material and the cost of increasing capacity from 30 to $50 \mathrm{~kg} /$ year. This proposal will be submitted in February 2002 for fiscal year 2003 (October 2002-September 2003) .

\section{ECP MOU Page 1}




\section{Future Production Costs and Schedules}

The ECP has provided estimates of costs for increasing production substantially. The first possible increase, up to $100 \mathrm{~kg} / \mathrm{year}$, is estimated to cost $\$ 3.5 \mathrm{M}$. A second option would increase production up to $200 \mathrm{~kg} / \mathrm{year}$ at a cost of about $\$ 5 \mathrm{M}$. Either option would require a year to effect after receipt of funding and would result in material costing about $\$ 45 \mathrm{k} / \mathrm{kg}$.

\section{Unresolved Issues}

1. Mode of transportation: Since Ge-68 grows in at about 1 atom per kg per day at sea level and posibly 100 times that at airliner altitude, the Collaboration may opt to ship by ground/sea. In this case ECP agrees to ship to the Russian port of exit within the quotes given above.

2. Contracting Mechanism: The Collaboration, ITEP, and ECP are considering the most efficient contracting mechanism and has no final solution at this time. Contracts with ITEP, ECP, the ISTC and otehr possible options will be compared in the near future.

3. It is possible that the product of the ECP may be processed into zone refined metal by another institution within Russia. ITEP and ECP will explore this possibility.

\section{Disposition}

This document will be presented to members of the Collaboration for discussion as well as the US Department of Energy and the US National Science Foundation for fact-finding regarding enrichment for double-beta decay and other experiments, and to the Russian Ministry of Atomic Energy. 
Appendix 2. Decay Chain Data Simplified Decay Scheme for ${ }^{238} \mathbf{U}$

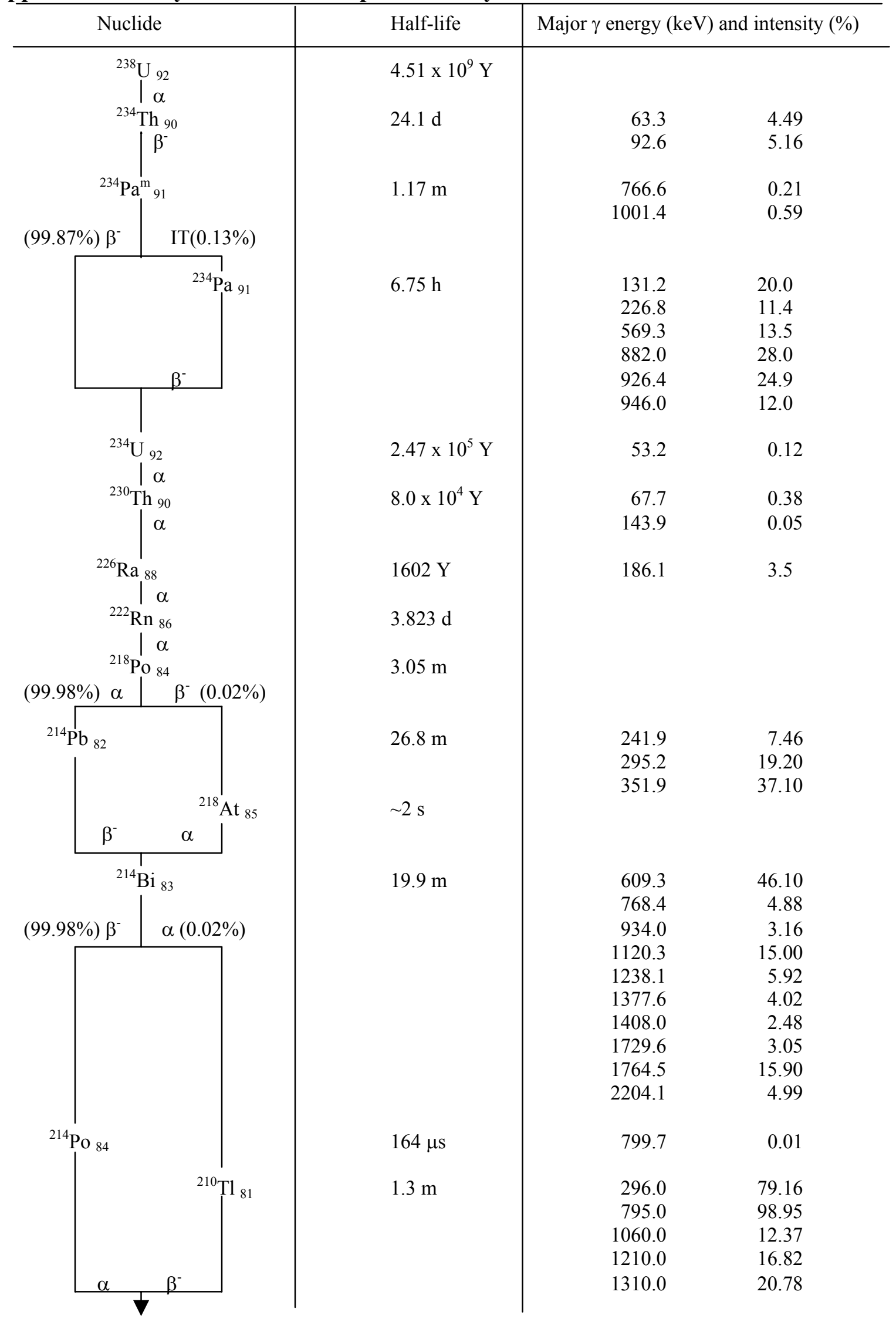

Draft

Page 81 


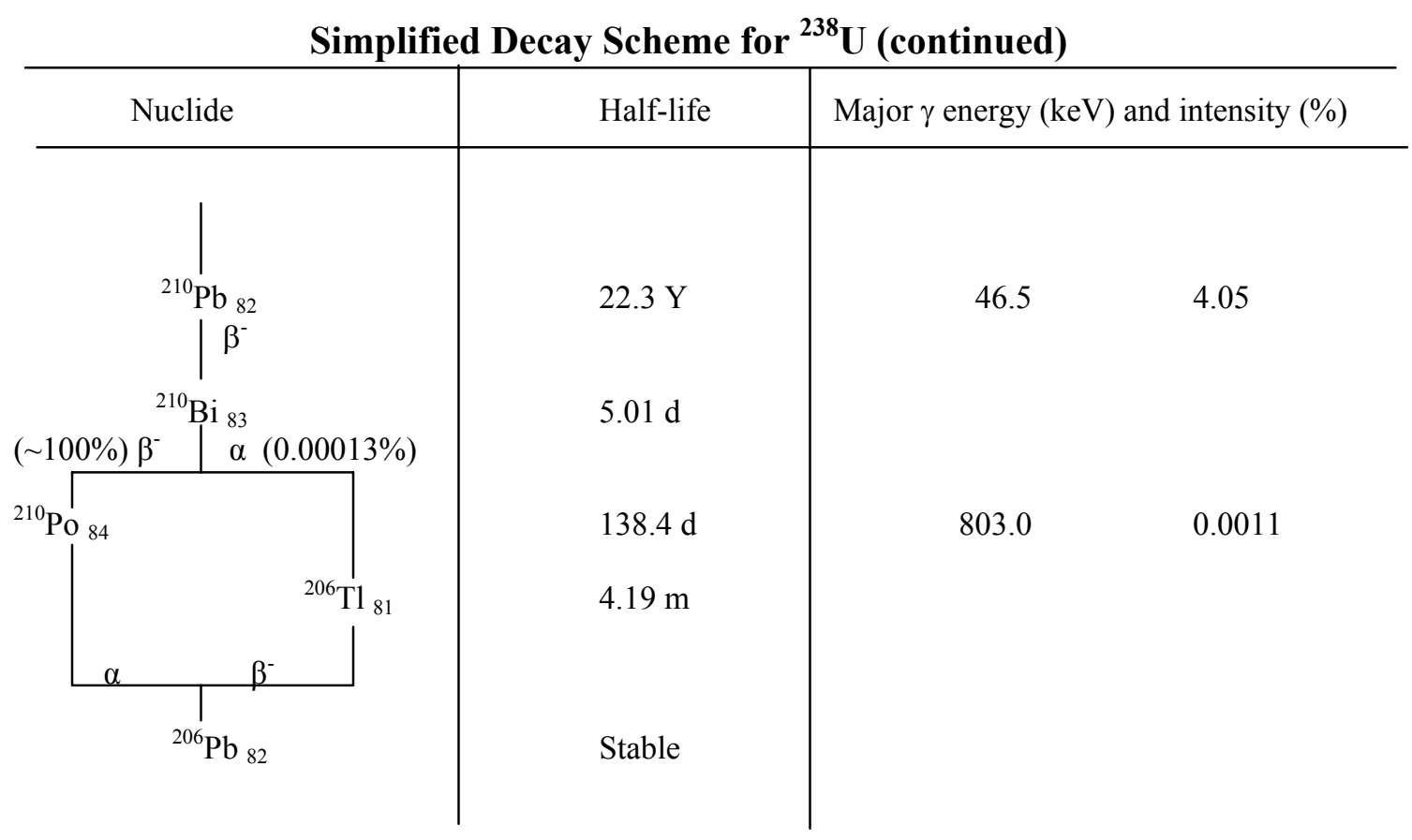




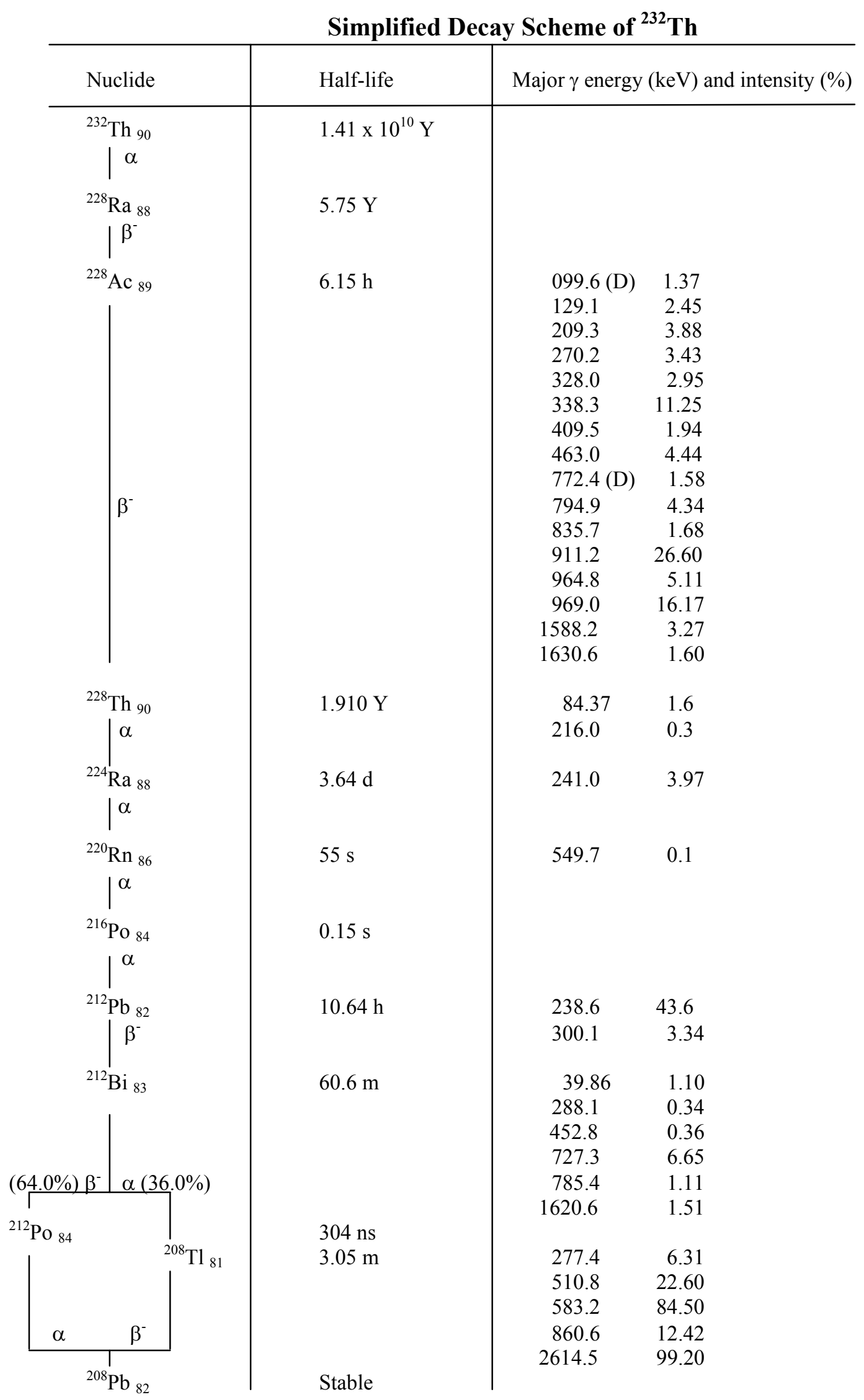

Draft

Page 83 


\section{Simplified Decay Scheme for ${ }^{235} U$}

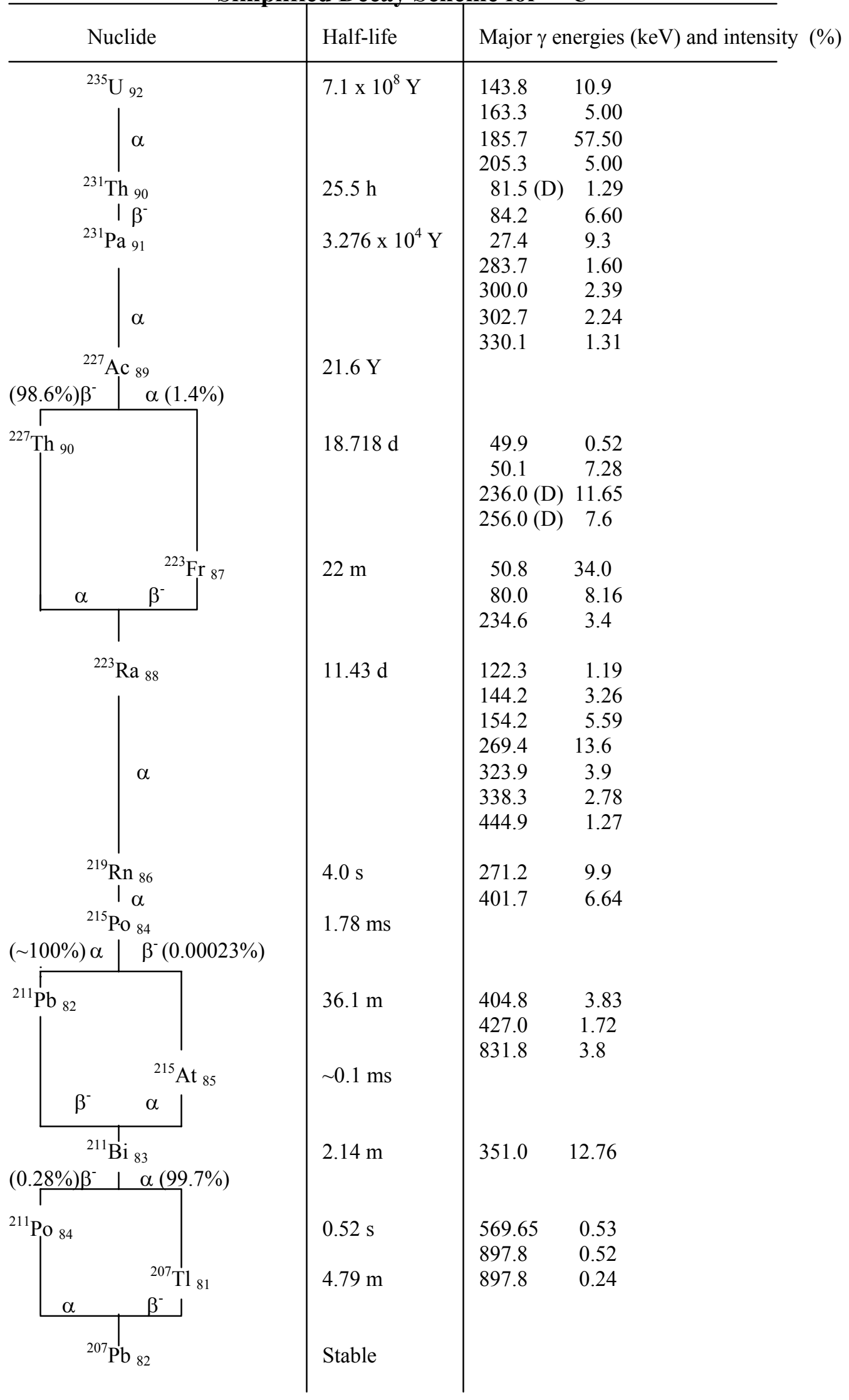




\subsection{Investigator Bios / Current and Pending Support}

We include here short biographical sketches of the investigators and document current and pending support. 


\section{Craig Edward Aalseth \\ Pacific Northwest National Laboratory}

\section{Education}

- Doctor of Philosophy in Physics from the University of South Carolina

- Bachelor of Science in Physics from South Dakota School of Mines and Technology

\section{Recent Positions}

- Pacific Northwest National Laboratory (PNNL) (December 2000 - present)

Postdoctoral Research Fellow

Continued development of radiation detector signal-processing methods, resulting in pending patent application. Applied new techniques to novel scintillators $\left(\mathrm{LiBaF}_{3}: \mathrm{Ce}\right)$, ${ }^{3} \mathrm{He}$ neutron detectors, and fast-neutron sensitivity with germanium ionization spectrometers. Developed multi-dimensional analysis codes $(\mathrm{C}++)$ for coincidence counting systems.

- Pacific Northwest National Laboratory (January 1998 - December 2000)

Graduate Research Fellow

Developed signal-processing methods for germanium ionization spectrometers. Techniques developed include event-localization and interaction-multiplicity discriminators. Screened ultra-low background components and developed thermallystable, ultra-low-background front-end FET amplifier modules for the International Germanium Experiment (IGEX). Modeled preamplifier performance (SPICE), charge collection, and electric fields for experimental ${ }^{76} \mathrm{Ge}$ detector systems. Developed complete detector simulation software chain (FORTRAN, C++).

- University of South Carolina

Graduate Teaching Assistant (September 1993 - December 1997)

Taught undergraduate laboratory classes, freshman and sophomore-level recitation and problem-solving sessions. Gave senior-level relativity and introductory quantummechanics lectures during frequent travel by dissertation advisor. Conducted review and problem-solving sessions for senior-level undergraduates.

- Pacific Northwest National Laboratory (May 1990 - August 1993)

International Germanium Experiment (IGEX) On-Site Technician

Reporting to IGEX collaborators at PNNL, was responsible for routine operation, calibration, data collection, and initial data reduction for state-of-health monitoring of the experiment. Worked at the 4850-ft-underground level of the Homestake gold mine in Lead, SD.

\section{Computing/Electronics Skills}

- Programming: C++, FORTRAN, SPICE, MathCad, Linux, ROOT.

- Techniques: Digital filtration, FFT, optimal filters, finite-element analysis.

- Hardware: Charge-integrating preamplifiers, data acquisition systems, low-noise techniques.

\section{Selected Recent Publications}

Craig Edward Aalseth and Harry S. Miley. A Novel Method of Pulse Shape Discrimination for Germanium Spectrometers. Nuclear Physics B - Proceedings Supplements, 87(1-3):491-492, June 2000. 
Craig Edward Aalseth, Frank T. Avignone, III, J. I. Collar, E. Garca, et al. The status of the IGEX ${ }^{76}$ Ge double-beta decay experiment in 1997. Nuclear Physics B, Proceedings Supplements, 70:236-238, January 1999.

Craig Edward Aalseth, Frank T. Avignone, III, J. I. Collar, E. Garca, et al. Neutrinoless double-beta decay of ${ }^{76} \mathrm{Ge}$ : First results from the International Germanium Experiment (IGEX) with six isotopically enriched detectors. Physical Review C (Nuclear Physics), 59(4):2108-13, April 1999.

Craig Edward Aalseth and Harry S. Miley. A simulation method for germanium pulse shapes. In 1999 IEEE Nuclear Science Symposium. Conference Record., pages 769-71.

Craig Edward Aalseth, Richard J. Arthur, and James H. Reeves. An improved low-background cooled FET assembly for germanium spectroscopy. In 1999 IEEE Nuclear Science Symposium. Conference Record., pages $378-81$.

Craig Edward Aalseth, Frank T. Avignone, III, Ronald L. Brodzinski, Harry S. Miley, and James H. Reeves. Using pulse shape discrimination to sort individual energy deposition events in a germanium crystal. Journal of Radioanalytical and Nuclear Chemistry, 233(1-2):119-123, 1998.

Craig Edward Aalseth, Frank T. Avignone, III, J. I. Collar, E. Garca, et al. Recent results from the IGEX double-beta decay experiment. Nuclear Physics B, 48:223-5, May 1996. Proc. Supp.

Craig Edward Aalseth, Frank T. Avignone, III, J. I. Collar, E. Garca, et al. Double-beta decay of ${ }^{76}$ Ge-the International Germanium Experiment (IGEX) in 1996. In 28th International Conference on High-Energy Physics, pages 1259-61, 1996. 


\section{Dale N. Anderson}

Pacific Northwest National Laboratory

Dale N. Anderson is a staff scientist at the Pacific Northwest National Laboratory in Richland, Washington. He received his $\mathrm{Ph}$. D. from the University of California at Riverside. He is currently a principal investigator for PNNL mathematics research in the US DOE Nuclear Explosion Monitoring program. Dr. Anderson served as the program coordinator for the Mathematics and Statistics program at Washington State University Tri-Cities for 6 years. He has 10 years experience in discrimination and time series applications, including project work in seismology, nuclear science, system reliability and general engineering applications. Dr. Anderson's expertise and interests include statistical classification, time series modeling, probability modeling and stochastic simulation. Relevant publications include (complete list available on request):

Statistical Issues in Seismic Monitoring. DN Anderson, KK Anderson, SR Sain and CJ Young, PNL-10678, U.S. Dept. of Energy, Pacific Northwest Laboratories, July, 1995.

A Probability Model: Tritium Release Into the Coolant of a Light Water Tritium

Production Reactor. DN Anderson., PNL-8079., U.S. Dept. of Energy, Pacific Northwest Laboratories., April, 1992.

"Centered Cauchy Conditionals." DN Anderson and BC Arnold., Communications in Statistics - Theory and Methods., 20(9), 1991, pp. 2881-2889.

"Linnik Distribution and Processes." DN Anderson and BC Arnold., Journal of Applied Probability., 30, 1993, pp. 330-340.

“A Multivariate Linnik Distribution.” DN Anderson., Statistics \& Probability Letters., 14, 1992, pp. 333-336.

"Modelling Gas Release Event Behaviour in Hazardous Waste Tanks", DN Anderson and BC Arnold, Journal of Environmental and Ecological Statistics, 3, 1996, 281-290.

"Discrimination Information in Phase Amplitude Thresholds with Application to Western China Regional Data", DN Anderson, SR Taylor and KK Anderson, Bulletin of the Seismological Society of America, 1999, 89, 3, pp. 648-656.

"Application of Regularized Discrimination Analysis to Regional Seismic Event Identification", DN Anderson and SR Taylor, Bulletin of the Seismological Society of America, To Appear. 


\section{AVIGNONE, Frank T., III \\ University of South Carolina}

Education

B.S. in Physics, Georgia Institute of Technology $(\tau \beta \pi, \Sigma \pi \Sigma, \Phi K \Phi)$

M.S. in Physics, Georgia Institute of Technology 1962

Ph. D. in Physics, Georgia Institute of Technology 1965

Employment History

Georgia Institute of Technology, Instructor $\quad$ 1962-1965

University of South Carolina, Assistant Professor $\quad$ 1965-1968

University of South Carolina, Associate Professor $\quad$ 1968-1973

University of South Carolina, Professor $\quad$ 1973-Present

International Centre for Theoretical Physics Trieste, Italy 1974

Oak Ridge National Laboratory, Visiting Scientist $\quad$ 1975, 1976

Georgia Institute of Technology, Visiting Professor 1976-1979

University of South Carolina, Chairman, Department of Physics and Astronomy 1979-1998

University of South Carolina, First Carolina Professor of Physics and Astronomy 1985-1998

Carolina Endowed Professor of Physics and Astronomy 1998-Present

Other History

National Defense, Education, and Welfare Fellowship, (GA Tech.) 1960-1963

National Science Foundation Graduate Research Fellowship, (GA Tech.) 1963-1965

Oak Ridge Institute of Nuclear Studies, Summer Fellowship 1965

Oak Ridge Associated Universities, Councilor for the University of South Carolina 1979-1998

Chairman, Executive Committee, Universities Isotope Separator, Oak Ridge 1974-1976

Recipient of the Russell Research Award in Science and Engineering (USC) 1972

Deputy Director of Technology Mobilization Program at the Office of the

Chief of Naval Research

1984-1986

Awarded Navy Commendation Medal by Chief of Naval Research 1984

Director of the Technology Mobilization Program at the Office of the Chief

of Naval Research

1986-1988

Awarded Meritorious Service Medal by Secretary of the Navy 1988

Recipient of the Russell Research Award in Science and Engineering (Second Award) 1991

Chairman, Southeastern Section of the American Physical Society 1991

Awarded 2nd Meritorious Service Medal by Commander and Chief, Atlantic Fleet

Elected to Fellowship in the American Physical Society 1991

Awarded the Jesse W. Beams Medal of the American Physical Society

"for significant Research in Physics" 1994

Received the Governor's Award for Excellence in Science 1995

Named Spokesman of the Oak Ridge Large Neutrino Detector Collaboration 1998

Elected to the Board of Directors, Oak Ridge Associated Universities $\quad$ 1998-Present

Awarded a Fulbright Scholar Award to Italy 2001

\section{Leadership Experiences}

Chairman of the Department of Physics and Astronomy at the University of South Carolina from 1 May 1979 to 30 June, 1998. During this period the department grew to the status claiming three of its five disciplines had reached international recognition. Federal funding grew by more than a factor of 12 . Sixteen of the present twenty four faculty were hired during that period.

Spokesman of the International Germanium Experiment IGEX. This has become one of the two most sensitive double beta decay experiments ever performed. It continues to develop new technology for next generation experiments. IGEX is a collaboration of the University of South Carolina, the Pacific 
Northwest National Laboratory, the Institute for Theoretical and Experimental Physics (Moscow) and the Baksan Neutrino Observatory, in the Kabardino Balkarian Republic in Russia, and the University of Zaragoza in Spain. It is performed in the Canfranc Underground Laboratory in Spain.

Spokesman of the SOLAX axion search experiment in the Hiparsa Iron Mine in Sierra Grande, Argentina. This experiment utilized a new technique involving the coherent Primakoff conversion of solar axions into Photons when the angle of incidience of the sun's axions satisfied a Bragg condition in the diamond structure of a germanium detector. It produced bounds on the axions mass and coupling significantly better than previous laboratory experiments. SOLAX was a collaboration of: The University of South Carolina, the Pacific Northwest National Laboratory, the TANDAR Laboratory in Buenos Aires, Argentina, The University of Zaragoza, Spain, The University of Thessaloniki, Greece, Tel Aviv University, Israel, and CERN.

Spokesman of the DEMOS, Cold dark matter search in the Hiparsa Iron Mine in Sierra Grande, Argentina. This experiment searched for Cold Dark Matter Candidates using a Ge detector using the annual modulaton technique, and for the first time the diurnal modulation of WIMP signal due to the scattering of WIMPs of a certain range in masses and couplings in the matter in the earth. This was a collaboration of the University of South Carolina, the University of Zaragoza, the Pacific Northwest National Laboratory, (PNNL), and the TANDAR accelerator laboratory in Buenos Aires.

Spokesman of the PNNL-USC low background Cold Dark Matter (CDM) Search, which was the first published terestial experimental search for CDM. It eventually eliminated Dirac Neutrinos as dominant candidates for Cold Dark Matter. This was a collaboration of the University of South Carolina, The Pacific Northwest National Laboratory (PNNL), The Harvard Smithsonian Center for Astrophysics, and Boston University. This seminal experiment was the first such effort and launched an industry of literally dozens of experiments using a variety of experimental techniques.

In 1998, elected Spokesman of the ORLaND collaboration, the prime goal of which is to build an underground bunker, adjacent to the first target station of the $\$ 1.4$ billion Spallation Neutron Source (SNS), to house a neutrino research laboratory. This project, the Oak Ridge Laboratory for Neutrino Detectors (ORLaND), will cost $\$ 60$ million. It is being proposed by a collaboration of 20 universities and institutes, the Oak Ridge National Laboratory, and the Oak Ridge Associated Universities.

As a Naval Officer, Captain F.T. Avignone: Created the Navy's Technology Mobilization Program under the Chief of Naval Research and served as its Deputy Director and its Director. Commanded Mine-Division 126, consisting of four ocean-going mine sweepers (MSO's), and later commanded Mine Squadron-22, an organization of 12 mine hunters and units in coastal cities from Boston to Galvaston, with a complement of 600 personnel.

Coordinator and Project Director of the University of South Carolina, Department of Defense three year grant, (\$9.3 million) for Combat Readiness. This was an interdisciplinary Program involving Chemistry, Biology, Mathematics, Computer Science, Statistics, and Marine Science.

Principal Investigator and Project Director of the \$2 million grant from the Department of Energy for academic support of the "Accelerator Production of Tritium" program. This included basic science applications of the facility-neutrino research, for example.

\section{Publications}

210 Publications 


\section{Alexander Stepanovich Barabash \\ Institute of Theoretical and Experimental Physics (Moscow, Russia)}

\section{Education}

- Moscow Engineering Physicsl Institute (1975)

- Candidate of Physics and Mathematics (1983)

- Doctor of Physics and Mathematics (second Russion step) (1999)

\section{Recent Positions}

- Institute of Theoretical and Experimental Physics (January 1986 - present)

Research Physicist (January 1986 - June 1989)

Senior Research Physicist (July 1989 - June 1991)

Leading Research Physicist (July 1991 - June 1997)

Head of Weak Interaction Laboratory (July 1997 - present)

- Institute for Nuclear Research (April 1975 - December 1985)

Engineer (April 1975 - June 1976)

Research Physicict (July 1976 - December 1985)

\section{Scientific Activities}

- development of liquid ionization detectors technique

- development of low-background detectors

- neutrino physics

- double beta decay

Selected Recent Publications ( one book and about 200 publications)

- Liquid Ionization Detectors, A.S. Barabash and A.I. Bolozdynya, Energoatomizdat, Moscow, 1993.

- Two neutrino double-beta decay of Mo-100 to the first excited 0+ state in Ru-100, A.S. Barabash et al., Physics Letters B345, 408-413 (1995).

- Two-neutrino double-beta decay Mo-100, D. Dassie et a., Physical Review D51, 2090 2100 (1995).

- Double-beta decay ob Cd-116, R. Arnold et al., Zeitscrift fur Physik C72, 239-247 (1996).

- Double beta decay of Se-82, R. Arnold et al., Nuclear Physics A636, 209-223 (1998).

- $\quad 2 v \beta \beta$ decay of Mo-100 to the first $0+$ excited state in Ru-100, Physics of Atomic Nuclei 62, 2211-2216 (1999).

- Double beta decay of Zr-96, R. Arnold et al., Nuclear Physics A658, 299-308 (1999).

- Limits on different Majoron decay modes of Mo-100, Cd-116, Se-82 and Zr-96 for neutrinoless double beta decay in the NEMO-2 experiment, R.Arnold et al., Nuclear Physics A678, 341-352 (2000).

- Is the weak interaction constant realy constant? A.S. Barabash, European Physical Journal A8, 137-140 (2000).

- Measurement of the $\beta \beta$-decay rate of Mo-100 to the first excited $0+$ state of Ru-100, L. De Braeckeler et al., Physical Review Letters 86, 3510-3513 (2001). 


\section{Theodore W. Bowyer \\ Pacific Northwest National Laboratory}

\section{Professional Experience}

1995-Present

Staff Scientist

Pacific Northwest National Laboratory

1999 - Present

Senior Research Scientist 1995-1999

- Principle investigator/project manager for nuclear explosion monitoring via automated radioactive xenon gas sampler-analyzer (ARSA) - for DOE's support of the Comprehensive Test Ban Treaty (CTBT). Lead nuclear detection/analysis, separations research, and engineering \& software development for $\sim 15$ scientists, engineers, technicians, post-docs, and students

- Received Federal Laboratory Consortium (FLC) award February 2001 for radionuclide measurement technology for the CTBT; semi-finalist - Discover Awards

1993-1995 Pacific Northwest National Laboratory Richland, WA

Post Doctoral Fellow

- Technical leader automated xenon gas sampler-analyzer (ARSA).

- Provided technical support to several nuclear radiation detection projects

Education

1987-1993 Indiana University Bloomington, IN

- Ph.D., M.S., Nuclear Physics - Measurement of $n-p$ Spin Observables Near $200 \mathrm{MeV}$

- Performed basic nuclear physics research - used and built radiation detection equipment

- Received William P. Koss award for top graduate student in physics research

1983-1987 University of Michigan -Flint Flint, MI

- B.A., Physics and Mathematics (Honors Program, Double Major)

\section{General Areas of Research}

Nuclear detection systems and analysis, nuclear explosion monitoring, non-proliferation and environmental measurements, electronics and instrumentation, novel detection systems, separations technology (gas collection, gas chromatography, cryogenic distillation, pressure swing), ultra-high vacuum technology, cryogenics, ion-implanted silicon based and scintillating fiber-based beta detection, Cerenkov radiation-based detection, low power gamma-ray detection, low power cryogenics

\section{Selected Recent Publications}

TW Bowyer, C Schlosser, KH Abel, M Auer, JC Hayes, TR Heimbigner, JI McIntyre, ME Panisko, PL Reeder, H Satorius, J. Schulze, W Weiss, "Detection and Analysis of Xenon Isotopes for the Comprehensive Nuclear-Test-Ban Treaty International Monitoring System", in press, J. of Environ. Radioactivity (2001).

TW Bowyer, B.D. Geelhood, T.W. Hossbach, R. Hansen, W.A. Wilcox, "In-situ, High Sensitivity, Measurement of Strontium-90 in Ground Water Using Cerenkov Light", Nucl. Instr. and Meth., A441, 577 (2000). 
TW Bowyer, KH Abel, CW Hubbard, ME Panisko, PL Reeder, RC Thompson, RA Warner, "Field Collection and Measurement of Radioxenon for the Comprehensive Test Ban Treaty", J. of Radioanalytical and Nucl. Chem., 240, 109 (1999).

S.M. Bowyer and T.W. Bowyer, "A Silicon Array Detector for High energy Betas", Nucl Instr. and Meth. A422, 837 (1999).

SW Wissink, SM Bowyer, WA Franklin, K Jiang, W Schmidt, J. Sowinski, EJ Stephenson, SP Wells, C Yu , "Spin Transfer in pp Elastic Scattering at $198 \mathrm{MeV}$ : Implications for the $\pi \mathrm{NN}$ Coupling Constant”, Phys. Rev. Lett., 83, 4498-4501, (1999).

T.W. Bowyer, K.H. Abel, C.W. Hubbard, A.D. McKinnon, M.E. Panisko, R.W. Perkins, P.L. Reeder, R.C. Thompson, R.A. Warner, "Automated separation and measurement of radioxenon for the Comprehensive Test Ban Treaty", J. of Radioanalytical and Nucl. Chem., 235, 77 (1998).

T.W. Bowyer, R. W. Perkins, K. H. Abel, W.K. Hensley, C. W. Hubbard, A. D. McKinnon, M. E. Panisko, P. L. Reeder, R. C. Thompson, and R. A. Warner, "Xenon Radionuclides, Atmospheric: Monitoring", The Encyclopedia of Environmental Analysis and Remediation, John Wiley \& Sons, Inc., 1998.

P. L. Reeder, T. W. Bowyer, and R. W. Perkins, "Xe Isotope Detection and Discrimination Using Beta Spectroscopy With Coincident Gamma Spectroscopy, Nucl. Instr. and Meth. A408, 582 (1998).

P. L. Reeder and T. W. Bowyer. "Delayed Coincidence Technique for ${ }^{133 g}$ Xe Detection", Nucl. Instr. and Meth. A408, 573 (1998).

A.J. Peurrung, T.W. Bowyer, R.A. Craig, P.L. Reeder, "Expected atmospheric concentration of ${ }^{42}$ Ar", Nucl. Instr. Meth. A396, 425 (1997).

T.W. Bowyer, K.H. Abel, W.K. Hensley, M.E. Panisko, R.W. Perkins, R.C. Thompson, “Ambient

${ }^{133}$ Xe Levels in the Northeastern US”, J. of Environ. Radioactivity, 37(2), 143 (1997). 


\section{Ronald Lee Brodzinski \\ Pacific Northwest National Laboratory}

\section{Education}

- Doctor of Philosophy in Nuclear Chemistry from Purdue University

- Bachelor of Science in Chemistry from Purdue University

\section{Employment}

- Pacific Northwest National Laboratory (December 1967 - present) Senior Staff Scientist Over 34 years experience conducting, directing and managing scientific investigations in nuclear chemistry and physics, nuclear detector development, and application of these techniques to the areas of environmental research, lunar and space sciences, cosmic radiation and cosmic-ray produced radionuclides, controlled thermonuclear reactor materials research, nuclear waste management, decontamination and decommissioning activities, environmental restoration and management, measurement and accountability of special nuclear materials, high energy spallation and fission studies, experimental fundamental particle physics, nuclear properties, cosmology, and astrophysics. Developed specialized detection systems for all types of radiations, alpha, beta, gamma, and neutron, and for detecting the interactions of all types of radiations with matter, including fast and thermal neutron activation with both prompt and delayed detectors. Principal Investigator for NASA Lunar Science Program making measurements on the lunar environment and conducting biological experiments on astronauts in space. Project manager for instrument development efforts to measure lepton nonconserving neutrinoless double-beta decay and spin dependent cosmion interactions, to nondestructively assay radioactive waste packages, to assay radioactive and heavy element concentrations throughout the environment, to make quantitative analyses of the transuranic activities in high level liquid wastes, and to develop many kinds of specialized radiation detectors and x-ray fluorescence spectrometers. Technical director of two international consortia on fundamental particle physics and resolution of Grand Unification.

Selected Publications (411 total publications)

- R. L. Brodzinski, D. P. Brown, J. C. Evans, Jr., W. K. Hensley, J. H. Reeves, N. A. Wogman, F. T. Avignone, III, and H. S. Miley, "The ${ }^{76}$ Ge Double Beta Decay Experiment at Homestake," Solar Neutrinos and Neutrino Astronomy, AIP Conference Proceedings No. 126, 50, 1985.

- R. L. Brodzinski, D. P. Brown, J. C. Evans, Jr., W. K. Hensley, J. H. Reeves, N. A. Wogman, F. T. Avignone, H. S. Miley, and R. S. Moore, "A Large-Volume Ultralow Background Germanium-Germanium Coincidence/Anticoincidence Gamma-Ray Spectrometer," Proceedings of the Fifth International Conference on Nuclear Methods in Environmental and Energy Research, p. 118, CONF-840408 (DE84017348), April, 1984.

- R. L. Brodzinski, D. P. Brown, J. C. Evans, Jr., W. K. Hensley, J. H. Reeves, N. A. Wogman, F. T. Avignone, III, and H. S. Miley, "An Ultralow Background Germanium Gamma-Ray Spectrometer," Nucl. Instrm. Methods Phys. Res. A239, 207 (1985).

- R. L. Brodzinski, J. H. Reeves, F. T. Avignone, and H. S. Miley, "The Impact of Natural Radioactivity in Solder on Low Background Experiments," Nuclear Instruments and Methods A254, 472 (1987).

- A. K. Drukier, S. P. Ahlen, F. T. Avignone, III, R. L. Brodzinski, K. Freese, G. Gelmini and D. N. Spergel, "Invisible Cosmic Rays," Proceedings NATO School of Cosmic Ray Astrophysics, Erice, Italy (1986).

- S. P. Ahlen, F. T. Avignone, III, R. L. Brodzinski, A. K. Drukier, G. Gelmini, and D. N. Spergel, "Limits on Cold Dark Matter Candidates from an Ultralow Background Germanium Spectrometer," Phys. Lett. B 195, 603 (1987). 
- R. L. Brodzinski, "Ultrasensitive Investigation of Neutrinoless and Two Neutrino Double-Beta Decay of ${ }^{76} \mathrm{Ge}, "$ Summaries of Research in High Energy Physics, DOE/ER-0353, November 1987.

- R. L. Brodzinski, J. H. Reeves, F. T. Avignone, III, and H. S. Miley, "Achieving Ultralow Background in a Germanium Spectrometer," J. Radioanal. Nucl. Chem. 124, 513 (1988).

- R. L. Brodzinski, H. S. Miley, J. H. Reeves, and F. T. Avignone, III, "Further Reduction of Radioactive Backgrounds in Ultrasensitive Germanium Spectrometers," Nucl. Instr. and Meth. A292, (1990) 337.

- R. L. Brodzinski, "Low-Level Gamma-Ray Spectrometry," J. Physics G: Nucl. Part. Phys. 17(Supplement), S403 (1991).

- A. S. Barabash, F. T. Avignone, III, R. L. Brodzinski, J. I. Collar, C. K. Guerard, H. S. Miley, J. H. Reeves, and V. I. Umatov, "The Soudan ${ }^{100}$ Mo Double-Beta Decay Experiment: A Status Report," PNL-SA-20607, Nuclear Physics B (Proc. Suppl.) 28A, 236 (1992).

- R. L. Brodzinski, H. S. Miley, J. H. Reeves, and F. T. Avignone, III, "Ultra-Low Background Germanium Spectrometry: Techniques and Results," PNL-SA-19755, Nuclear Physics B (Proc. Suppl.) 28A, 415 (1992).

- E. García, F. T. Avignone III, R. L. Brodzinski, J. I. Collar, H. S. Miley, A. Morales, J. Morales, R. Núñez-Lagos, J. Puimedón, J. H. Reeves, , C. Sáenz, and J. A. Villar, "Dark Matter Searches with a Germanium Detector at the Canfranc Tunnel," Nuclear Physics B (Proc. Suppl.) 28A, 286 (1992).

- A. K. Drukier, F. T. Avignone, III, R. L. Brodzinski, J. I. Collar, G. Gelmini, H. S. Miley, A. Morales, J. H. Reeves, and D. Spergel, "Progress Report on the Search for Cold Dark Matter Using Ultralow-Background Germanium Detectors at Homestake," PNL-20606, Nuclear Physics B (Proc. Suppl.) 28A, 293 (1992).

- J. I. Collar, F. T. Avignone, III, R. L. Brodzinski, E. Garcia, H. S. Miley, A. Morales, J. Morales, R. Nuñez-Lagos, J. H. Reeves, C. Saenz, and J. A. Villar, "Bounds on Diurnal Modulations from the Cosme-II Dark Matter Experiment," PNL-20571, Nuclear Physics B (Proc. Suppl.) 28A, 297 (1992).

- R. L. Brodzinski, H. S. Miley, J. H. Reeves, and F. T. Avignone (III), "Low-Background Germanium Spectrometry: The Bottom Line," PNL-SA-18693, J. Radioanal. Nucl. Chem. 160, 355 (1992).

- A. S. Barabash, F. T. Avignone III, C. K. Guerard, R. L. Brodzinski, H. S. Miley, J. H. Reeves, and V. I. Umatov, "Two Neutrino Double-Beta Decay of ${ }^{100}$ Mo to the First Excited $0^{+}$State in ${ }^{100} \mathrm{Ru}$," Proceedings of the International Symposium on Weak and Electromagnetic Interactions in Nuclei, WEIN 92, Dubna, June 16-22, 1992.

- R.L. Brodzinski, F.T. Avignone, J.I. Collar, H. Courant, E. Garcia, C.K. Guerard, W.K. Hensley, I.V. Kirpichnikov, H.S. Miley, A. Morales, J. Morales, R. Núñez-Lagos, S.B. Osetrov, V.S. Pogosov, A.A. Pomansky, J. Puimedón, J.H. Reeves, K. Ruddick, C. Sáenz, A. Salinas, M.L. Sarsa, A.A. Smolnikov, A.S. Starostin, A.G. Tamanyan, S.I. Vasiliev, and J.A. Villar, "Status Report on the International Germanium Experiment," Nucl.Phys. B (Proc. Supl.) 31, 76(1993).

- J. I. Collar, F. T. Avignone, III, R. L. Brodzinski, E. García, H. S. Miley, A. Morales, J. Morales, R. Núñez-Lagos, J. Puimedón, J. H. Reeves, C. Sáenz, A. Salinas, M. L. Sarsa, and J. A. Villar, "Remarks on Direct Searches for Cold Dark Matter Candidates," Nucl. Phys. B (Proc. Suppl.) 31, 377 (1993).

- A. S. Barabash, F. T. Avignone III, C. K. Guerard, R. L. Brodzinski, H. S. Miley, J. H. Reeves, and V. I. Umatov, "First Results from the Soviet-American Experiment on Double-Beta Decay of ${ }^{100}$ Mo to the Excited States of ${ }^{100} \mathrm{Ru}$," Proceedings of the XXVIth Rencontre de Moriond, Massive Neutrinos Tests of Fundamental Symmetries, p. 77 (1991).

- E. García, A. Morales, J. Morales, M. L. Sarsa, A. Ortiz de Solórzano, E. Cerezo, R. Núñez-Lagos, J. Puimedón, C. Sáenz, A. Salinas, J. A. Villar, J. I. Collar, F. T. Avignone, R. L. Brodzinski, H. S. Miley, and J. H. Reeves, "Results of a Dark Matter Search with a Germanium Detector in the Canfranc Tunnel," Phys. Rev. D 51, 1458 (1995).

- A. S. Barabash, F. T. Avignone III, J. I. Collar, C. K. Guerard, R. J. Arthur, R. L. Brodzinski, H. S. Miley, J. H. Reeves, J. R. Meier, K. Ruddick, and V. I. Umatov, "Two Neutrino Double-Beta Decay of ${ }^{100}$ Mo to the First Excited $0^{+}$State in ${ }^{100} \mathrm{Ru}$," Phys. Letts. B 345, 408 (1995). 
- R. L. Brodzinski, H. S. Miley, J. H. Reeves, and F. T. Avignone, "Low-Background Germanium Spectrometry - The Bottom Line Three Years Later," J. Radioanal. Nucl. Chem. 193, 61 (1995); Proceedings of the Third International Conference on Methods and Applications of Radioanalytical Chemistry I, 435, (1995).

- Y. Aharonov, F. T. Avignone III, R. L. Brodzinski, J. I. Collar, E. García, H. S. Miley, A. Morales, J. Morales, S. Nussinov, A. Ortiz de Solórzano, J. Puimedóon, J. H. Reeves, C. Sáenz, A. Salinas, M. L. Sarsa, and J. A. Villar, "New Experimental Limits for the Electron Stability," Phys. Letts. B 353, 168 (1995).

- Y. Aharonov, F. T. Avignone III, R. L. Brodzinski, J. I. Collar, E. García, H. S. Miley, A. Morales, J. Morales, S. Nussinov, A. Ortiz de Solórzano, J. Puimedón, J. H. Reeves, C. Sáenz, A. Salinas, M. L. Sarsa, and J. A. Villar, "New Laboratory Bounds on the Stability of the Electron," Phys. Rev. D 52, 3785 (1995).

- D. E. Di Gregorio, D. Abriola, F. T. Avignone III, R. L. Brodzinski, J. I. Collar, H. A. Farach, E García, A O. Gattone, F. Hasenbalg, H. Huck, H. S. Miley, A. Morales, J. Morales, A. Ortiz de Solórzano, J. Puimedón, J. H. Reeves, C. Sáenz, A. Salinas, M. L. Sarsa, D. Tomasi, I. Urteaga, and J. A. Villar, "Looking for Daily Modulation Effects of WIMPs in the Southern Hemisphere," Nucl. Phys. B (Proc. Suppl.) 48, 56 (1996).

- D. Abriola, F. T. Avignone III, R. L. Brodzinski, J. I. Collar, D. E. Di Gregorio, H. A. Farach, E. García, A. O. Gattone, F. Hasenbalg, H. Huck, H. S. Miley, A. Morales, J. Morales, A. Ortiz de Solórzano, J. Puimedón, J. H. Reeves, C. Sáenz, A. Salinas, M. L. Sarsa, D. Tomasi, I. Urteaga, and J. A. Villar, "Searching for Cold Dark Matter in the Southern Hemisphere. The Experiment at Sierra Grande," Astropart. Phys. 6, 63 (1996).

- O. Gattone, D. Abriola, F. T. Avignone, R. L. Brodzinski, J. I. Collar, R. J. Creswick, D. E. Di Gregorio, H. A. Farach, C. K. Guérard, F. Hasenbalg, H. Huck, H. S. Miley, A. Morales, J. Morales, S. Nussinov, A. Ortiz de Solórzano, J. H. Reeves, J. A. Villar, and K. Zioutas, "Experimental Search for Solar Axions," Nucl. Phys. B (Proc. Suppl) 70 (1999) 59.

- M. L. Sarsa, F. T. Avignone, R. L. Brodzinski, E. Cerezo, J. I. Collar, E. Garcia, J. H. Reeves, H. S. Miley, A. Morales, J. Morales, R. Nunez-Lagos, A. O. de Solorzano, J. Puimedon, C. Saenz, A. Salinas, and J. A. Villar, "Dark-Matter Searches at the Canfranc Tunnel," Nucl. Phys. B Suppl. 35 (1994) 154.

- A. A. Klimenko, S. B. Osetrov, A. A. Smolnikov, S. I. Vasilyev, F. T. Avignone, R. L. Brodzinski, H. S. Miley, and J. H. Reeves, "Search for Annual and Daily Dark-Matter Modulations with Ge Detectors at Baksan," Phys. Atom. Nucl. 61 (1998) 1129.

- D. Abriola, F. T. Avignone, R. L. Brodzinski, J. I. Collar, D. E. DiGregorio, H. A Farach, E. Garcia, A. O. Gattone, C. K. Guerard, F. Hasenbalg, H. Huck, H. S. Miley, A. Morales, J. Morales, A. O. de Solorzano, J. Puimedon, J. H. Reeves, A. Salinas, M. L. Sarsa, and J. A. Villar, "Search for an Annual Modulation of Dark-Matter Signals with a Germanium Spectrometer at the Sierra Grande Laboratory," Astropart. Phys. 10 (1999) 133.

- R. Brodzinski, “The 'Discovery' of Alpha Activity in Lead and Solder,” J. Electron. Mater. 29 (2000) 1294.

- D. Gonzalez, C. E. Aalseth, F. T. Avignone, R. L. Brodzinski, S. Cebrian, E. Garcia, W. K. Hensley, I. G. Irastorza, I. V. Kirpichnikov, A. A. Klimenko, H. S. Miley, A. Morales, J. Morales, A. O. de Solozano, S. B. Osetrov, V. S. Pogosov, J. Puimedon, J. H. Reeves, M. L. Sarsa, S. Scopel, A. A. Smolnikov, A. G. Tamanyan, A. A. Vasenko, S. I. Vasiliev, and J. A. Villar, "Current IGEX results for neutrinoless double-beta decay of Ge-76," Nucl. Phys. B-Proc. Sup. 87 (2000) 278.

- S. Cebrián, A. Morales, C. E. Aalseth, F. T. Avignone III, R. L. Brodzinski, E. García, D. González, W. K Hensley, I. G. Irastorza, I. V. Kirpichnikov, A. A. Klimenko, H. S. Miley, J. Morales, A. Ortiz de Solórzano, S. B. Osetrov, V. S. Pogosov, J. Puimedón, J. H. Reeves, M. L. Sarsa, S. Scopel, A. A. Smolnikov, A. G. Tamanyan, A. A. Vasenko, S. I. Vasiliev, and J. A. Villar, "First results of the IGEX Dark Matter experiment at the Canfranc Underground Laboratory," Nucl. Phys. B (Proc. Suppl.) 95 (2001) 229. 


\author{
Juan I. Collar \\ Enrico Fermi Institute
}

$\begin{array}{lccl}\begin{array}{l}\text { Professional preparation } \\ \text { Universidad Autonoma de Madrid }\end{array} & \text { Physics } & 1985 & \text { B.S. (equivalent) } \\ \text { University of South Carolina } & \text { Physics } & 1992 & \text { Ph.D. } \\ \text { University of South Carolina } & \text { Exp. Physics } & 92-95 & \text { Postdoc } \\ \text { CERN } & \text { Exp. Physics } & 96-98 & \text { Fellow } \\ \text { Université Paris 6\&7 } & \text { Exp. Physics } & 98-01 & \text { Fellow }\end{array}$

Appointments

(Oct. 2001- )

$(1998-2001)$

(1996-1998)

(1992-1995)

(1986-1992)
Assistant Professor, Dept. of Physics, Enrico Fermi Institute, and the College University of Chicago, USA.

Marie Curie Fellow, G.P.S., Universite Paris VII

Scientific Associate, EP Division, CERN

Fellow of the Experimental Physics Division

European Organization for Nuclear Research (CERN) Geneva, Switzerland.

Fellow of the Fondation Robert Schuman

Groupe de Physique des Solides

Universités Paris VII/VI, Paris, France.

Postdoctoral Research Associate

Department of Physics and Astronomy, U. of South Carolina

$\underline{\text { Research \& Teaching Assistant }}$

Department of Physics and Astronomy, U. of South Carolina

Selected relevant publications (from a total of 104. Full list available from http://collar.home.cern.ch/collar/pubs.pdf):

J.I. Collar, A new approach to neutrino and WIMP detection using telecom-grade electrooptic and fiber-optic components, submitted to Nucl. Instr. Meth. A (hepex/0105015)

J.I. Collar and Y. Giomataris, Possible Low-Background Applications of MICROMEGAS Detector Technology, Nucl. Instr. Meth. 471 (2001) 254 (hep-ex/0009063)

J.I. Collar, T. Girard, D. Limagne, H.S. Miley, J. Puibasset and G. Waysand, First Dark Matter Limits from a Large-Mass, Low-Background Superheated Droplet Detector, Phys. Rev. Lett., 85 (2000) 3083 (astro-ph/0001511)

J.I. Collar and K. Zioutas, Exotic Heavily Ionizing Particles can be Constrained by the Geological Abundance of Fullerenes, Phys. Rev. Lett. 83 (1999) 3097 (astro-ph/9902310)

K. Zioutas et al., (26 authors, corresponding author J.I. Collar), A Decommissioned LHC Model Magnet as an Axion Telescope, Nucl. Instr. Meth. A425 (1999) 482 (astro-ph/9801176) 
C.E. Aalseth et al. (26 authors), Neutrinoless double-beta decay of Ge-76: First results from the International Germanium Experiment (IGEX) with six isotopically enriched detectors. Phys. Rev. C59 (1999) 2108.

J.I. Collar, Solar-Bound Weakly Interacting Massive Particles: A No-Frills Phenomenology, Phys. Rev. D59 (1999) 063514 (astro-ph/9808058)

F.T. Avignone III et al. (19 authors), Experimental Search for Solar Axions Via Coherent Primakoff Conversion in a Germanium Spectrometer, Phys. Rev. Lett. 81 (1998) 5068 (astro-ph/9708008)

J.I. Collar, Superheated Microdrops as Cold Dark Matter Detectors, Phys. Rev. D54 (1996) R1247 (rapid communications) (astro-ph/9607150)

A.S. Barabash et al. (11 authors), Two Neutrino Double-Beta Decay of Mo-100 to the First Excited 0+ State in Ru-100, Phys. Lett. B345 (1995) 408.

E. Garcia et al. (16 authors), Results of a Dark Matter Search With a Germanium Detector in the Canfranc Tunnel, Phys. Rev. D51 (1995) 1458.

H.S. Miley, F.T. Avignone III, R. Brodzinski, J.I. Collar and J. Reeves, Suggestive Evidence for the Two-Neutrino Double-Beta Decay of Ge-76, Phys. Rev. Lett. 65 (1990) 3092.

\section{Collaborators}

This partial list does not include numerous collaborators at CERN (the P.I. has been involved in several large experiments there). Only the most representative collaborators can be mentioned in this limited space: Y. Aharonov (USC), F.T. Avignone III (USC), R. Brodzinski (PNNL), L.Chadderton (Australian Natl. University), R.J. Creswick (USC), D.E. Di Gregorio (TANDAR, Argentina), H.A. Farach (USC), T.A. Girard (Lisbon), A.O. Gattone (TANDAR, Argentina), I. Giomataris (CEA/Saclay, France), P. Gorham (JPL), V. Jeudy (Paris), I.V.Kirpichnikov (ITEP, Russia), D. Limagne (Paris), J. Learned (Hawaii), H.S. Miley (PNNL), A. Morales (Zaragoza, Spain), J. Morales (Zaragoza, Spain), S. Nussinov (Tel Aviv), P. Pearle (Hamilton College), G. Raffelt (Max Planck, Germany), C. Rubbia (CERN), J.A.Rubio (CERN), A.S. Starostin (ITEP, Russia), G. Waysand (Paris), L.C.L. Yuan (SRRC, Taiwan), K. Zioutas (Thessaloniki \& CERN)

Graduate advisor: Frank T. Avignone III (USC).

Graduate Students Advised: Joel Puibasset (Université Paris VI, Ph.D. received Dec. 2000, Summa Cum Laude and special mention by the Jury); Tomoko Morlat (Université Paris VI, in progress). 


\section{Peter Doe \\ University of Washington \\ Center for Experimental Nuclear Physics and Astrophysics}

\section{Professional Profile}

Experimental nuclear physics, in particular the study of weak interactions, solar and supernova neutrinos. Extensive experience in the development of nuclear instrumentation and detectors to study the above phenomena.

Education: Ph.D. Physics, Durham University, UK 1977.

M.Sc. $\quad$ Physics, Durham University, UK 1975.

B.Sc. $\quad$ Physics, London University, UK 1971

\section{Professional Experience}

University Of Washington, Seattle, WA

Research Professor

Los Alamos National Laboratory, Los Alamos, NM

Staff Member

University Of California Irvine, Irvine, CA

Research Associate
July, 1994 - Present

Jan, 1990 - July, 1994

Jan, 1978 - Jan, 1990

\section{Sample Refereed Publications (Of 38 Referred Publications)}

- "Measurement of Charged Current Interactions Produced by ${ }^{8}$ B Solar Neutrinos at the Sudbury Observatory", Q.R. Ahmad et al, (178 authors), Phys. Rev. Lett. 87, 071301 (2001).

- "Measurement of Interference Between W and Z Exchange in Electron-Neutrino Electron Scattering" R.C. Allen, H.H. Chen, P.J. Doe, R. Hausammann, W.P. Lee, H.J. Mahler, M.E. Potter, X.Q. Lu, K.C. Wang, T.J. Bowles, R.L. Burman, R.D. Carlini, D.R.F. Cochran, J.S. Frank, E. Piasetzky, V.D. Sandberg, D.A. Krakauer, and R.L. Talaga, Phys. Rev. Lett. $\underline{64}$, 1330 (1990).

- "Measurement of the Exclusive Cross Section ${ }^{12} C\left(v_{e}, e^{-}\right){ }^{12} N($ g.s. $) "$

- R. C. Allen, H.H. Chen, P.J. Doe, R. Hausammann, W.P. Lee, H.J. Mahler, M.E. Potter, X.Q. Lu, K.C. Wang, T.J. Bowles, R.L. Burman, R.D. Carlini, D.R.F. Cochran, J.S. Frank, E. Piasetzky, V.D. Sandberg, D.A. Krakauer, and R.L. Talaga, Phys. Rev. Lett. 64, 1871 (1990).

- "First Observation and Cross-Section Measurement of $v_{e}+e^{-} \rightarrow v_{e}+e^{-}$" R. C. Allen, H.H. Chen, P.J. Doe, R. Hausammann, W.P. Lee, H.J. Mahler, M.E. Potter, X.Q. Lu, K.C. Wang, T.J. Bowles, R.L. Burman, R.D. Carlini, D.R.F. Cochran, J.S. Frank, E. Piasetzky, V.D. Sandberg, D.A. Krakauer, and R.L. Talaga, Phys. Rev. Lett. 55, 2401, (1985).

- "Observation of Tracks in a Two Dimensional Liquid Argon Time Projection Chamber" P.J. Doe, H.J. Mahler, H.H. Chen, Nucl. Instr. \& Meth., 199, 639, (1982). 


\section{Steven Ray Elliott \\ University Of Washington}

\section{Professional Profile}

Experienced Physicist in areas encompassing atomic, nuclear and particle physics. Knowledgeable in processes which require understanding the integration of these subfields. Effective in working with students in the laboratory and classroom.

Education: Ph.D. Physics, University of California, Irvine 1987.

Dissertation: "Double Beta Decay in ${ }^{82} \mathrm{Se}^{\circ}$.

M.S. $\quad$ Physics, University of California, Irvine 1984.

B.S. Physics, magna cum laude, University of New Mexico 1982.

\section{Professional Experience}

University Of Washington, Seattle, WA

Research Associate Professor, Research Assistant Professor

Lawrence Livermore National Laboratory, Livermore, CA Oct, 1991 - Dec, 1994

Postdoctoral Appointment

Los Alamos National Laboratory, Los Alamos, NM $\quad$ Feb, 1991 - Aug, 1991

Term Staff Member

Los Alamos National Laboratory, Los Alamos, NM Jan, 1988 - Jan, 1991

Postdoctoral Appointment

\section{Sample Refereed Publications (Over 40 Referred Publications)}

- "Direct Evidence for Two-Neutrino Double-Beta Decay in ${ }^{82} \mathrm{Se} "$ S. R. Elliott, A. A. Hahn, and M. K. Moe, Phys. Rev. Lett. 59, 2020-2023 (1987).

- "Search for Double Beta Decay in ${ }^{100}$ Mo and $92 \mathrm{Mo"}$ S. R. Elliott, A. A. Hahn, and M. K. Moe, Phys. Rev. C36 No. 5, 2129-2131 (1987).

- "A Time Projection Chamber for Detection of Double Beta Decay" S. R. Elliott, A. A. Hahn, and M. K. Moe, Nucl. Instr. and Meth. A273, 226-239 (1988).

- "Double Beta Decay", S. R. Elliott, A. A. Hahn, and M. K. Moe, Phys. Bull. 39, 227 (1988).

- "Double Beta Decay of ${ }^{82}$ Se" S. R. Elliott, A. A. Hahn, M. K. Moe, M. A. Nelson, and M. A. Vient, Phys. Rev. C46, 1535-1537 (1992).

- "Measurement of the Solar Neutrino Capture rate with Gallium Metal" J. N. Abdurashitov et al., (25 authors),Phys. Rev. C60, 055801, 31 pages, (1999).

- "Measuring Supernova Neutrino Temperatures Using Lead Perchlorate" S. R. Elliott, Phys. Rev. C62, 065802 (2000).

- "Measurement of Charged Current Interactions Produced by ${ }^{8}$ B Solar Neutrinos at the Sudbury Neutrino Observatory", Q.R. Ahmad et al., (178 authors), Phys. Rev. Lett. 87, 071301 (2001). 


\section{Degrees}

St John's College, Oxford University

St John's College, Oxford University
Richard J. Gaitskell

Brown University

Physics

Physics

BAHons/MA Jul1985

D. Phil Nov1993

\section{Appointments}

Brown University, Assistant Professor 2001-

University College London, University Lecturer 2000-2001

Visiting Scholar, Stanford University $1996-2000$

Center Fellow, Center for Particle Astrophysics, UC Berkeley 1995-2000

Lindemann Fellowship (Lindemann Trust Committee, UK) 1995-1998

Senior Dean of Arts, Magdalen College

1994-1995

Physics Tutor, Magdalen College, Oxford

$1993-1995$

Fellowship by Examination, Magdalen College, Oxford Univ.

$1993-1995$

Post Doctoral Research Fellowship,

1993-1995

Particle Physics and Astronomy Research Council

College Lecturer (Stipendary), Trinity College, Oxford 1992-1993

Physics Tutor, Mansfield College, Oxford

1990-1992

Graduate Studentship, Science and Engineering Research Council

1989-1993

Open Scholarship, St John's College, Oxford

1981

Scholarship, Worshipful Company of Scientific Instrument Makers,

1981

\section{Recent Publications}

R J Gaitskell, Toward One Tonne Direct WIMP Detectors: Have We Got What It Takes? , 3rd International Workshop on Identification of Dark Matter (World Scientific, September 2001). (Preprint available at http://xxx.lanl.gov/abs/astro-ph/0106200.)

R Abusaidi et al. [CDMS Collaboration], Results on the WIMP-Nucleon Cross Section from the Cryogenic Dark Matter Search (CDMS), Phys. Rev. Lett. 84 (2000) 5699

R.M. Clarke, P.L. Brink, B. Cabrera, P. Colling, M.B. Crisler, A.K. Davies, S. Eichblatt, R.J. Gaitskell , J. Hellmig , J.M. Martinis, S.W. Nam, T. Saab, and B.A. Young Enhanced ballistic phonon production for surface events in cryogenic silicon detectors, Appl. Phys. Lett. 76 (2000) 2958

D. Tovey, R. Gaitskell, P. Gondolo, Y. Ramachers, and L. Roszkowski, A New Method for Presenting ModelIndependent Spin-Dependent Cross-Section Limits from Dark Matter Searches, Phys. Lett. B 488 (2000) 17

J. Hellmig, R. Gaitskell, R.A. Abusaidi, B. Cabrera, R.M. Clarke, J. Emes, S.W. Nam, T. Saab, B. Sadoulet, D. Seitz, B.A. Young, CDMS II Z-Sensitive Ionization and Phonon Germanium Detector, NIM A 444 (2000) 308

T. Saab, R.M. Clarke, B. Cabrera, R.A. Abusaidi, R. Gaitskell, Design of QET Phonon Sensors for the CDMS Zip Detectors NIM A 444 (2000) 300.

S. Golwala et al., [CDMS Collaboration] Exclusion limits on the WIMP-nucleon scattering cross-section from the Cryogenic Dark Matter Search Experiemnt, (ibid) p 345

R. J. Gaitskell, et al. [CDMS Collaboration], Latest Results on the WIMP-Nucleon Cross Section from the Cryogenic Dark Matter Search (CDMS), Proceedings of TAUP99 Conference, September 1999, Paris, France

M.R. Hauser, R. Gaitskell, and J.P. Wolfe, Imaging Nonequilibrium Phonons in Nb Phys. Rev. B 60 (1999) 3072 (http://ojps.aip.org/getabs/servlet/GetabsServlet?prog=normal\&id=PRBMDO000060000005003072000001)

M.R. Hauser, R. Gaitskell, and J.P. Wolfe, Imaging Nonequilibrium Phonons in Nb, Physica B 263 (1999) 87

S. Golwala, et al., [CDMS Collaboration] Hunting for WIMPs with the Cryogenic Dark Matter Search, Proceedings of the 19th Texas Symposium on Relativistic Astrophysics, Paris, France, 14-18 December 1998. 


\section{Other Significant Publications}

L.C. Angrave, N.E. Booth, R.J. Gaitskell, G.L.Salmon and M.R. Harston, Measurement of the Atomic Exchange Effect in Nuclear $\beta$ Decay, Phys. Rev. Lett., 80 (1998) 1610.

R.J. Gaitskell, et al., Performance of $165 \mathrm{~g}$ Ge BLIP Detectors in CDMS Experiment, ed. S. Cooper, Proceedings of the VIIth International Workshop on Low Temperature Detectors, July 1997, Munich, Germany (MPI, Munich 1997) 221-223

R.J. Gaitskell, L.C. Angrave, N.E. Booth, A.D. Hahn, G.L.Salmon and A.M.Swift, A measurement of the beta spectrum of Ni-63 using a new type of cryogenic detector, Nucl. Instrum. and Methods A 370 (1996) 250

R.J. Gaitskell, L.C. Angrave, N.E. Booth, E. Esposito, T.J. Giles, C. Höss, E.P. Houwman, G.L. Salmon, M. van den Putte and S. Wänninger, The design of a cryogenic dark matter detector based on the detection of the recoil direction of target nuclei, ibid. p.162

R.J. Gaitskell, L.C. Angrave, N.E. Booth, A.D. Hahn, G.L.Salmon and A.M.Swift, A measurement of the beta spectrum of Ni-63 using a new type of cryogenic detector, Phys Lett B 370 (1996) 163

R.J. Gaitskell, P.D. Barnes, Jr., A. Da Silva, B. Sadoulet and T. Shutt, The Statistics of Background Rejection in Direct Detection Experiments for Dark Matter, Nucl. Phys. B (Proc.Suppl.), 51B (1996) 279.

R J Gaitskell, L C Angrave, N E Booth, A D Hahn, G L Salmon and A M Swift, A Measurement of the Beta Spectrum of $63^{3}$ i using a New Type of Calorimetric Cryogenic Detector, eds., P. J. Bussey, I. G. Knowles, 27th International Conference on High Energy Physics (IOP Publishing, Glasgow, UK, 1994).

R. J. Gaitskell, A. D. Hahn, N. E. Booth, G. L. Salmon and D. L. Kazakovtsev, Modelling the thermalisation of non-equilibrium phonon distributions in bulk single crystals, J. Low Temp. Phys. 93, 683 (1993).

N. E. Booth, P. L. Brink, R. J. Gaitskell, D. J. Goldie, A. D. Hahn, G. L. Salmon and A. M. Swift, Low temperature detectors for elementary particles, Physica B 197, 39 (1994).

R. J. Gaitskell, N. E. Booth and G. L. Salmon, in Low Temperature Detectors for Neutrinos and Dark Matter IV, eds. N. E. Booth, G. L. Salmon, (Editions Frontières, Gif-sur-Yvette, France, 1992), p. 435.

\section{Other Activities}

"Into the AntiWorld" I prepared and delivered lecture on physics, which followed West End Theater (Bloomsbury) production (4 nights, May 2001) about PAM Dirac and antimatter entitled "Into the AntiWord" (First performed at CERN in 1998). Audience was made up of general public. My lecture was designed to flesh out the physics (that was mentioned in the play) of antimatter, and also describe the role of antimatter in particle physics and cosmology. A group of physicists (which I lead) then had additional informal discussions with the public in the theater after the production, the session typically lasting 1-2 hours.

http://dmtools.berkeley.edu (web site). Since early 1999 I have maintained a web site that collects both theoretical and experimental results in the Particle Dark Matter (WIMP) direct detection field. The site features an interactive plotter that allows users to select data sets to combine on single plots that are generated dynamically on the web. This underlying data is also made available in a standard form/database to users. The site has an extensive bibliography of references in the field.

Co-organized a 2 week long workshop at CERN in summer 1999 (Dark Matter Tools) that was designed to bring SUSY theorists and experimentalists from all the active WIMP search groups together in a more informal atmosphere. The goals were to establish standard calculation framework for data analysis and display amongst the experimental groups. An additional goal was to better integrate work of SUSY accelerator-based theorists so that it is directly relevant to experimental searches. This would allow easier comparison of accelerator SUSY data and those from dark matter searches. We have followed up original meeting with a number of additional workshop groups, piggy-backing on larger conferences, to ensure that this work continued.

If assisted in extensive Center for Particle Astrophysics (CfPA, UC Berkeley) education/outreach program. Regularly (1995-1998) contributed written material and illustrations to web site (http://cfpa.berkeley.edu) that was designed for non-specialists to get a better idea of our work in Particle Astrophysics and Dark Matter.

http://cdms.berkeley.edu \& http://cdms.berkeley.edu/cdms restricted/ (web sites) Established CDMS Collaboration web site in 1995 to better ensure transmission of information to parties interested in the CDMS experiment. Internal web site facilitate information flow between eight institutions involved in experiment, and now acts as an invaluable database of experimental data, findings plus acting as an historical record of progress. 


\section{David Victor Jordan}

Pacific Northwest National Laboratory

\section{Education}

- Ph.D. in Experimental Nuclear Physics, Massachusetts Institute of Technology, 1994. Prof. William Bertozzi, supervisor.

- B.S., Physics, University of Washington, 1987

\section{Professional History}

- Pacific Northwest National Laboratory, Richland, WA (Sep. 2001 - present) Senior Research Scientist (Level 1)

Responsible for the conduct of research and development in the area of nuclear non-proliferation and disarmament. Special emphasis on computational and modeling support for detector development.

- TRIUMF, Vancouver, BC (Nov. 1996 - Aug. 2001)

Postdoctoral fellow

Responsible for analysis and simulation of data in TRIUMF experiment E704, $\mathrm{np} \rightarrow \mathrm{d} \pi^{0}$ near threshold. Developed data analysis software for track reconstruction from SASP spectrometer focal plane's Vertical Drift Chambers; simulated deuteron data using GEANT detector simulation software.

- Saskatchewan Accelerator Laboratory, Saskatoon, SK (Jan. 1994 - Oct. 1996)

Postdoctoral fellow

Responsible for instrumentation and commissioning of facility's QDD electron spectrometer. Developed data acquisition and analysis software for the spectrometer's focal plane detectors.

\section{Publications}

- A separation of the longitudinal and transverse structure functions in the $\mathrm{D}(\mathrm{e}, \mathrm{e}$ 'p $) \mathrm{n}$ reaction, D. Jordan, T. McIlvain, et al., Physical Review Letters 76, 1579 (1996).

- Charge Symmetry Breaking in the reaction $\mathrm{np} \rightarrow \mathrm{d} \pi^{0}$, A.K. Opper, E.G. Auld, et al., Nuclear Physics A663, 505 (2000).

- Out-of-plane measurements of the fifth response function of the exclusive electronuclear response, S.M. Dolfini, R.O. Alarcon et al., Physical Review C60, 064622 (1999).

- Measurement of the $\gamma \mathrm{p} \rightarrow \pi^{+} \mathrm{n}$ reaction near threshold, E. Korkmaz, N.R. Kolb et al., Physical Review Letters 83, 3609 (1999).

- Measurement of the interference structure function $\mathrm{R}_{\mathrm{LT}}$ for the ${ }^{12} \mathrm{C}(\mathrm{e}, \mathrm{e}$ ' $\mathrm{p})$ reaction in the quasielastic region, M. Holtrop, D. Jordan, et al., Physical Review C58, 3205 (1998). 


\section{Richard Thomas Kouzes}

Pacific Northwest National Laboratory

\section{Education}

- Doctor of Philosophy in Physics from Princeton University

- Master of Arts in Physics from Princeton University

- Bachelor of Science in Physics from Michigan State University

\section{Recent Positions}

- Pacific Northwest National Laboratory (March 2000 - present)

$\underline{\text { Senior Staff Scientist }}$

As a senior staff scientist (Level 6) in the Chemical and Radiological Sciences Group of the National Security Division (NSD) of PNNL, responsible for the conduct of research and development in the area of nuclear non-proliferation and disarmament. I work on issues related to the implementation of the bilateral, trilateral, and START agreements, especially in the authentication of attribute measurement systems.

- Washington State University

Adjunct Professor of Physics (June 2000 - present)

- West Virginia University

Director Program Development Science, Engineering \& Medicine (June 1995-March 2000)

Professor of Physics (June 1995 - May 2000)

Research Integrity Officer (June 1995 - March 2000)

Adjunct Professor of Physics (June 2000 - present)

- Pacific Northwest National Laboratory (November 1991 - June 1995) Group Leader

- Princeton University Physics Department (August 1976 - November 1991)

Senior Research Physicist and Lecturer (June 1987 - November 1991)

Research Physicist (indefinite term 1983) and Lecturer (June 1980 - June 1987)

Research Staff and Lecturer (August 1976 - June 1980)

Wilson College Faculty Fellow and Advisor (August 1982 - November 1991)

\section{Computing}

- Collaboratories and computer supported cooperative work

- Scientific computation and visualization

- Data acquisition systems from bench top to large implementations

\section{Selected Professional Memberships And Activities}

- Southeastern Universities Research Association (SURA) Trustee for WVU 1995-2000

- Aeronautics and Space Engineering Board's committee on Advanced Engineering Environments member (National Research Council panel - NASA funded) 1998-2000

- WVU Research Integrity Officer; act as a grievance judge 1996-2000

- Member of the DOE Energy Sciences Computing Coordinating Committee 1991-1999

- Chair of the DOE ESCC Applications Working Group 1992-1997

- Co-founder and former chairman, IEEE Committee for Computer Applications in Nuclear and Plasma Sciences

-WV Governor's Science \& Technology Council Information Technology Committee 
Selected Recent Publications (Over 140 total publications)

- Astrophysics Simulations, J.M.A. Danby, R.T. Kouzes, C.A. Whitney, J Wiley \& Sons, NY, (1995), Translated into Japanese (May 1996).

- Collaboratories: Scientists Working Together Apart, R.T. Kouzes, World and I Magazine (March 1995).

- Electronic Collaborative Tools, R.T. Kouzes, J.D. Myers, IEEE Real Time '95 Conference, East Lansing, MI (May 1995).

- Data Reflection Algorithm For Spectral Enhancement In Fourier Transform Icr And Nmr Spectroscopies, M.V. Gorshkov, R.T. Kouzes, Analytical Chemistry 67, 3412-3420 (October 1995).

- Collaboratories: Doing Science On The Internet, Richard T. Kouzes, James D. Myers, William A. Wulf, IEEE Computer 29, 40-46 (August 1996).

- Electronic Nose And Their Applications In Environmental Monitoring, S. Hashem, P.E. Keller, R.T. Kouzes, L.J. Kangas, Applications Of Neural Networks In Environment, Energy, And Health; Workshop Weann'95, Richland, WA, World Scientific, Singapore, 7481(1996).

- Collaboratories: Can We Work Together Apart?, R. Kouzes, Scientific Computing and Automation 14, 52-54 (January 1997).

- Collaboratories: Working Together Apart, R. Kouzes, Proceedings of 10th IEEE Real Time Conference, Beaune, France (September 22-26, 1997).

- Search For Neutrinos From The Sun Using The Reaction ${ }^{71} G a\left(v_{e}, e^{-}\right)^{71} G e$, A.I. Abazov, et. al., Physical Review Letters 67, 3332 (1991)

- Advanced Engineering Environments, R. Deemer, et. al., National Academy Press (June 1999) ISBN 0-309-06541-0.

- The Sudbury Neutrino Observatory, J. Boger, et. al., Nuclear Instruments and Methods A449, 172-207 (2000). 


\section{Harry S. Miley \\ Pacific Northwest National Laboratory}

\section{Employment/Education History:}

Staff Scientist, PNNL

Senior Research Scientist, PNNL

4/95-present

Adjunct Faculty, Washington State University

3/90-4/95

Research Scientist, PNNL

9/91-present

6/87-3/90

Ph.D. Physics, University of South Carolina

1987

Thesis: The Search for Double-Beta Decay of $76 \mathrm{Ge}$

B.S. Physics, South Carolina College

1982

(Honors College of the University of South Carolina)

\section{Technical Experience}

Radionuclide Aerosol Sampler/Analyzer: Led team of scientists and engineers on high-profile program $(\sim \$ 1.5 \mathrm{M} /$ year $)$ to develop new particulate air sampling technology to verify the Comprehensive Test Ban Treaty.

High-Sensitivity, Low-Profile Neutron Detector: Led team of scientists to rapidly develop a system for detection of plutonium diversion at traffic choke points. The sensor is constructed of a new type of scintillating fiber optics (Winner of 1999 R\&D100 Award) which are sensitive to thermal neutrons and gamma-rays.

Double-Beta Decay: Design and construction of several generations of PNNL-USC detectors with the lowest reported levels of radioisotopic contamination, six orders lower than typical low background detectors. In 1990 the PNNL-USC collaboration published a credible, subsequentlyconfirmed measurement of the two-neutrino decay mode halflife at about 1021 years. Recent developments include the creation of a novel pulse-shape analysis method for nuclear decays based on digital signal processors.

\section{Selected Publications}

- J I Collar, et al,, "Prospects for SIMPLE 2000: a large-mass, low-background superheated droplet detector for WIMP searches", New Journal of Physics 2 No. 1 (July 2000)

- L. D. Braeckeleer, et al, "Double Beta Decay and The Majorana Project, The Majorana Collaboration", Proceedings of Carolina Neutrino Symposium, (http://www.tunl.duke.edu/ ludwigdb/usc00.pdf)

- C. E. Aalseth, et al, "The Status of the IGEX 76Ge Double-Beta Decay Experiment in 1997," Nucl. Phys. B (Proc. Suppl.) 70 (1999) 236.

- O. Gattone et al, Experimental Search for Solar Axions," Nucl. Phys. B (Proc. Suppl) 70 (1999) 59.

- C. E. Aalseth, et al, "Using Pulse Shape Discrimination to Sort Individual Energy Deposition Events in a Germanium Crystal," J. Radioanal. Nucl. Chem. 233, 119 (1998).

- H. S. Miley, et al, "Monte Carlo Simulations of Low Background Detectors," J. Radioanal. Nucl. Chem. 193, 247 (1995)

- E. Garcia, et al, "Results of a Dark Matter Search with a Germanium Detector in the Canfrac Tunnel," Phys. Rev. D 51, 1458 (1995) 
- R. L. Brodzinski, H. S. Miley, J. H. Reeves, and F. T. Avignone, "Low-Background Germanium Spectrometry - The Bottom Line Three Years Later," J. Radioanal. Nucl. Chem. 193, 61 (1995)

- S. Barabash, F et al, "Two Neutrino Double-Beta Decay of 100Mo to the First Excited 0+ State in 100Ru," Phys. Letts. B 345, 408 (1995).

- Y. Aharonov, et al, "New Experimental Limits for the Electron Stability," Phys. Letts. B 353, 168 (1995).

- Y. Aharonov, et al, "New Laboratory Bounds on the Stability of the Electron," Phys. Rev. D 52, 3785 (1995).

- F. T. Avignone, et al, "The International Germanium Experiment (IGEX) in 1993," Nucl. Phys. B (Proc. Suppl.) 35, 354 (1994).

- H. S. Miley, et al, "Simulated Progress in Double-Beta Decay," Nucl. Phys. B (Proc. Suppl.) 35, 388 (1994).

- F. T. Avignone, et al, "Double-Beta Decay: Some Recent Results and Developments," Prog. Part. Nucl. Phys. 32, 223 (1994)

- R. L. Brodzinski, et al, "Status Report on the International Germanium Experiment," Nucl. Phys. B (Proc. Suppl.) 31, 76 (1993).

- J. I. Collar, et al, "Remarks on Direct Searches for Cold Dark Matter Candidates," Nucl. Phys. B (Proc. Suppl.) 31, 377 (1993).

- R. L. Brodzinski, et al, "Ultra-Low Background Germanium Spectrometry: Techniques and Results,", Nucl. Phys. B (Proc. Suppl.) 28A, 415 (1992).

- E. García, et al, "Dark Matter Searches with a Germanium Detector at the Canfranc Tunnel," Nuclear Physics B (Proc. Suppl.) 28A, 286 (1992).

- H. S. Miley, et al, "New Techniques and Results in 76Ge Double-Beta Decay," Nuclear Physics B (Proc. Suppl.) 28A, 212 (1992).

- K. Drukier, et al, "Progress Report on the Search for Cold Dark Matter Using UltralowBackground Germanium Detectors at Homestake," PNL-20606, Nuclear Physics B (Proc. Suppl.) 28A, 293 (1992).

- J. I. Collar, et al, "Bounds on Diurnal Modulations from the Cosme-II Dark Matter Experiment," PNL-20571, Nuclear Physics B (Proc. Suppl.) 28A, 297 (1992).

- R. L. Brodzinski, H. S. Miley, J. H. Reeves, and F. T. Avignone (III), "Low-Background Germanium Spectrometry: The Bottom Line," PNL-SA-18693, J. Radioanal. Nucl. Chem. 160, 355 (1992).

- F. T. Avignone, III, et al, "Theoretical and Experimental Investigation of Cosmogenic Radioisotope Production in Germanium," PNL-20590, Nuclear Physics B (Proc. Suppl.) 28A, 280 (1992).

- S. Barabash, et al, "The Soudan 100Mo Double-Beta Decay Experiment: A Status Report," PNLSA-20607, Nuclear Physics B (Proc. Suppl.) 28A, 236 (1992).

- H. S. Miley, R. L. Brodzinski, and J. H. Reeves, "Low-Background Counting Systems Compared," PNL-SA-18699, J. Radioanal. Nucl. Chem. 160, 371 (1992).

- F. T. Avignone III, et al, "Measurements of the Half-Life of the 2 Neutrino ??-Decay of 76Ge," J. Physics G: Nucl. Part. Phys. 17(Supplement), S181 (1991).

- F. T. Avignone, et al, "Confirmation of the Observation of Two Neutrino Double Beta Decay of 76Ge." Phys. Letts. B256: 559 (1991)

- H. S. Miley, F. T. Avignone, R. L. Brodzinski, J. I. Collar, and J. H. Reeves. 1990. "Evidence for the Double Beta Decay of 76Ge." Phys. Rev.Letts. 65: 3092. 
- R. L. Brodzinski, H. S. Miley, J. H. Reeves and F. T. Avignone. 1990. "Further Reduction of Radioactive Backgrounds in Ultra-Sensitive Germanium Spectrometers." NIM A292: 337.

- R. L. Brodzinski, J. H. Reeves, F. T. Avignone, III, and H. S. Miley, "Achieving Ultralow Background in a Germanium Spectrometer," J. Radioanal. Nucl. Chem. 124, 513 (1988).

- F. T. Avignone, et al,"Search for Axions from the 1115-keV Transition of 65Cu." Phys. Rev. D37:618 (1988)

- F. T. Avignone, R. L. Brodzinski, H. S. Miley, and J. H. Reeves. 1987. "Alternative Interpretation of the Results from the St. Gotthard Laboratory Double Beta Decay Experiment." Phys. Letts. B198: 253.

- F. T. Avignone, H. S. Miley, R. L. Brodzinski, and J. H. Reeves. 1987. "Analysis and Interpretation of a Large Body of 76Ge Zero-Neutrino Double Beta Decay Data." Phys. Rev. D35: 1713.

- F. T. Avignone, R. L. Brodzinski, J. C. Evans, W. K. Hensley, H. S. Miley, and J. H. Reeves. 1986. "Search for the Double Beta Decay of 76Ge." Phys. Rev. C34: 666.

- F. T. Avignone, W. C. Barker, and H. S. Miley. 1986. "Search for Anisotropic Directional Correlations Between g Rays and Kx Rays Emitted for 154Gd." Phys. Rev. A33: 4375.

- F. T. Avignone, R. L. Brodzinski, W. K. Hensley, H. S. Miley, and J. H. Reeves. 1986. "New Experimental Limit on the Stability of the Electron." Phys. Rev. D34: 97.

- F. T. Avignone, H. S. Miley, W. J. Padgett, and D. W. Weier. "Monte Carlo, Hypothesis Test for Rare Events Superimposed on a Background." NIM A234:315.

- F. T. Avignone, et al, "Ultralow-Background Study of Neutrinoless Double Beta Decay of 76Ge: New Limit on the Majorana Mass of Electron Neutrino." Phys. Rev. Letts. 54: 2309.

- R. L. Brodzinski, et al, "The 76Ge Double Beta Decay Experiment at Homestake," Solar Neutrinos and Neutrino Astronomy, AIP Conference Proceedings No. 126, 50, 1985.

- F. T. Avignone, et al. 1985. "Near Threshold Behavior of Pair-Production Cross Sections in a Lead Target." Phys. Rev. A32: 2622.

- R. L. Brodzinski, et al. 1985. "An Ultralow Background Germanium Gamma-Ray Spectrometer." NIM A239: 207. 


\section{William Karl Pitts \\ Pacific Northwest National Laboratory}

\section{Education:}

Ph.D., Physics

M.S., Physics

B.S., Physics, Chemistry, and Mathematics
Indiana University, Bloomington, IN 1987

Indiana University, Bloomington, IN 1982

Austin Peay State University 1980

Clarksville, TN

\section{Technical Employment History}

2000-Present

2001-Present

1996-2001

1997-Present

1992-1996

1988-1992

1987-1988

1980-1988

1978-1980
Senior Research Scientist II

Adjunct Professor, Physics

Associate Professor, Physics

Associate (Adjunct Faculty),

Assistant Professor, Physics

Research Scientist

Post-Doctoral Associate

Research Assistant

Analyst (Chemist)
PNNL, Richland WA

University of Louisville, Louisville, KY

University of Louisville, Louisville, KY

University of Louisville, Louisville, KY

Electrical Engineering

University of Louisville, Louisville, KY

University of Wisconsin, Madison, WI

University of Illinois, Urbana, IL

Indiana University, Bloomington, IN

Jersey Miniere Zine Co., Clarksville, TN

\section{Relevant Research, Teaching, and Management Experience:}

Twenty years experience with nuclear physics, nuclear detectors, microfabrication technology, and measurements, including

- Gas Proportional Detectors: Experienced with microfabricated gas detectors, including design, fabrication, operation, and analysis; developed new detector geometries for charged particle and X-ray detection.

- Microfabrication Experience: Together with students, developed microfabrication facilities and expertise for detector development at UofL. Led development of laser micromachining facility and application of laser micromachining to gas ionization detectors.

- Nuclear Physics Experience: General electronics, laboratory, vacuum, and computer skills developed during experiments at Indiana University and University of Wisconsin.

- Nuclear Material Measurements: Characterization measurements of SNM at the Hanford Site.

\section{Patents:}

“Optical Imaging System Utilizing A Charge Amplification Device," W. K. Pitts, K. M. Walsh, and K. Solberg (US Patent 5,602,397). 
"Radiation Detection Based Upon Charge Amplification in a Gaseous Medium," K. Solberg, W. K. Pitts, and K. M. Walsh (US Patent 5,614,722).

\section{Selected Publications:}

"Low Energy Charged Particle Detection in a Light-Ion Storage Ring," W. K. Pitts et al., Nucl. Instrum. and Meth $\underline{\text { A302 }} 382$ (1991).

"Spin dependence in $p p$ scattering in the Coulomb-nuclear interference region", W.K. Pitts et al., Phys. Rev. Cㄴ5 R1 (1992)

“Analyzing power measurements for $p$ - $d$ radiative capture", F. Goeckner, W.K.Pitts, and L.D. Knutson, Phys. Rev. C4도 R2536 (1992)

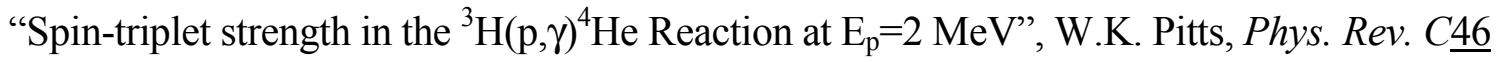
R15, (1992)

"Measurement of Spin Observables Using a Storage ring with Polarized Beam and Polarized Internal Gas Target," K. Lee et al, Phys. Rev. Lett. 70738 (1993).

"Measurement of Quasielastic ${ }^{3} \mathrm{He}(\mathrm{p}, \mathrm{pN})$ Scattering from Polarized ${ }^{3} \mathrm{He}$ and the Three-Body Ground State Spin Structure,” M. A. Miller et al., Phys. Rev. Lett. 74502 (1995).

"Search for the Production of Pionium Atoms Near Threshold," A. C. Betker et al., Phys. Rev. Lett. 773510 (1996).

“GEM: Performance and Aging Tests,” H. S. Cho et al., IEEE Trans. Nuc. Sci. $\underline{46} 306$ (1999).

"Development and Operation of Laser Machined Microwell Detectors," W. K. Pitts et al., Nucl. Instrum. And Methods A 438277 (1999).

"Effect of Well Diameter upon MicroWell Detector Performance," W. K. Pitts et al., IEEE Trans. Nuc. Sci. 47918 (2000).

"Experience with laser microfabricated detectors at the University of Louisville", W. K. Pitts and M.D. Martin, Nucl. Instrum. And Methods A 471268 (2001). 


\section{Robert Graham Hamish Robertson \\ University of Washington}

\section{Education}

- Ph.D. in Physics from McMaster University 1971

- M.A. in Natural Sciences from Oxford University 1971

-B.A. ( $1^{\text {st }}$ class honors) in Natural Sciences from Oxford University 1965

\section{Positions}

- University of Washington

Professor of Physics (1994 - present)

Scientific Director, Center for Experimental Nuclear Physics and Astrophysics (1997 - present)

- Los Alamos National Laboratory

Technical Staff Member (1981 - 1994)

Fellow (1985 - present)

- Michigan State University

Professor (1981)

Associate Professor of Physics (1978 - 1981)

Assistant Professor (1973 - 1978)

Research Assistant Professor (1972 - 1973)

Research Associate (1971 - 1972)

- Chalk River Nuclear Laboratories

Visiting Scientist (1980)

- Argonne National Laboratory

Visiting Scientist (1979)

- Princetion University

Research Associate (1974 - 1975)

\section{Honors and Awards}

- Fellow, Institute of Physics (London) 1998

- APS Tom W. Bonner Prize 1997

- Fellow, Los Alamos National Laboratory 1985

- Fellow, American Physical Society 1982

- Alfred P. Sloan Foundation Fellow 1976

\section{Service}

Organizing Committee, 6th International Conference on Atomic Masses, East Lansing, 1979

Review Panel, NSF-supported Tandem Laboratories, 1981

Review Panel, DOE-supported Heavy-lon Outside Users' Programs, 1982

Referee, Physical Review C and D, Physics Letters, Physical Review Letters, and Journal of Geophysical Research

Member, NSERC (Canada) Grant Selection Committee, 1983 - 1986

Member, LANL Postdoctoral Committee, 1983 - 1986

Colloquium Committee Chairman, Physics Division, LANL, 1982, 1988 - 92.

Spokesman for US SNO Collaboration on Capital Funding, 1989 -.

Member, NSAC Long-Range Plan Workshop, 1989

Member and Chair, Lawrence Berkeley Laboratory Nuclear Science Division Review Panel, 1986 1989

Group Leader for Neutral Current Detection, Sudbury Neutrino Observatory Project, 1990 -.

Member, LANL Centers Advisory Board, 1990.

Chair, LAMPF Electroweak Program Advisory Committee, 1990-1992.

Editorial Board, Physical Review D, 1992-5.

Member, Panel on Neutrino Astrophysics, National Academy of Sciences, 1993-4.

Convenor, American Physical Society Division of Particles and Fields Long-term Planning Study, $1994-5$.

Convenor, Snowmass Workshop on Neutrino Astrophysics and Cosmology, June 30 - July 131994.

Member, AIP Panel on Physical Review C, 1994 -5. 
Convenor, American Physical Society Division of Nuclear Physics Long-range Planning Study, 19945.

Member, Visiting Committee for the Division of Physics, Mathematics and Astronomy, CalTech, 1995-.

Member, HEPAP Subpanel on Long-Baseline Neutrino Experiments, 1995-6.

Chair, Nuclear Science Advisory Committee, 1995-6.

Member, Nuclear Science Advisory Committee, 1995-2001.

Member, Board on Physics and Astronomy, National Research Council, 1995-8.

Organizing Committee, INT Conference on Solar Fusion Rates, 1997.

Organizing Committee, ITP Conference on Astrophysics and Cosmology, 1997.

Member, APS Tom W. Bonner Prize Committee, 1997.

International Advisory Committee, ICHEP 98.

APS Centennial Speaker, 1998 - 9.

Chair, APS Division of Nuclear Physics, 2000.

Member, NSAC Intermediate Energy Physics Review Panel, 1998.

Member, Lehman Review, NUMI Long-baseline Neutrino Project. 1998

Member, NAS/NRC Committee on Physics of the Universe 2000 -.

Member, SAGENAP (Scientific Assessment Group for Experiments in Non-Accelerator Physics), 2000 -.

Selected Recent Publications (Over 55 total publications)

- "Limit on Electron Antineutrino Mass from Observation of the $\beta$ decay of Molecular Tritium," R. G. H. Robertson, T. J. Bowles, G. J. Stephenson, Jr., D. L. Wark, J. F. Wilkerson, and D. A. Knapp, Phys. Rev. Lett. 67, 957 (1991).

- "Probability of a Solution to the Solar Neutrino Problem within the Minimal Standard Model," Karsten M. Heeger and R.G.H. Robertson, Phys. Rev. Lett. 77, 3720 (1996).

- "Solar Fusion Cross Sections," R.G.H. Robertson with 37 other authors, Rev. Mod. Phys., 70,1265 (1998).

- "Low-background ${ }^{3} \mathrm{He}$ Proportional Counters for Use in the Sudbury Neutrino Observatory," M.C. Browne, T.J. Bowles, S.J. Brice, P.J. Doe, C.A. Duba, S.R. Elliott, E.I. Esch, M.M. Fowler, J.V. Germani, A. Goldschmidt, K.M. Heeger, A. Hime, K.T. Lesko, G.G. Miller, R.W. Ollerhead, A.W.P. Poon, R.G.H. Robertson, M.W.E. Smith, T.D. Steiger, R.G. Stokstad, P.M. Thornewell, J.B. Wilhelmy, J.F. Wilkerson, and J.M. Wouters; IEEE Transactions in Nuclear Science $\underline{46}$, pg. 873 (1999).

- "Solar Neutrino Interactions with ${ }^{18} \mathrm{O}$ in the SuperKamiokande Water Cerenkov Detector," W.C. Haxton and R.G.H.Robertson, Phys. Rev. C $\underline{59}, 515$ (1999).

- "A Compact ${ }^{3} \mathrm{H}(\mathrm{p}, \gamma)^{4} \mathrm{He}$ 19.8-MeV Gamma-Ray Source for Energy Calibration of the Sudbury Neutrino Observatory, A.W.P. Poon, R.J. Komar, C.E.Waltham, M.C. Browne, R.G.H. Robertson, N.P. Kherani, and H.B. Mak, Nucl. Instr. Methods in Physics Research A452, 115, 2000.

- "Spectroscopy of Double-Beta and Inverse-Beta Decays from ${ }^{100}$ Mo for Neutrinos", H. Ejiri, J. Engel, R. Hazama, P. Krastev, N. Kudomi, and R.G.H. Robertson, Phys. Rev. Lett. 85, 2917, 2000.

- "New Limit on the D Coefficient in Polarized Neutron Decay," L.J. Lising, S.R. Hwang, J.M. Adams, T.J. Bowles, M.C. Browne, T.E. Chupp, K.P. Coulter, M.S. Dewey, S.J. Freedman, B.K. Fujikawa, A. Garcia, G.L. Greene, G.L. Jones, H.P. Mumm, J.S. Nico, J.M. Richardson, R.G.H. Robertson, T.D. Steiger, W.A. Teasdale, A.K. Thompson, E.G. Wasserman, F.E. Weitfeldt, R.C. Welsh, and J.F. Wilkerson, Phys. Rev. C62 055501-1 2000.

- "Direct Measurements of Neutrino Mass," J.F. Wilkerson and R.G.H. Robertson, Chapter in 'Neutrino Physics,' edited by D.O. Caldwell, (Springer, 2001).

- "Measurement of the rate of $v_{e}+d \rightarrow p+p+e^{-}$interactions produced by ${ }^{8} B$ solar neutrinos at the Sudbury Neutrino Observatory" Q.R. Ahmad et al. (the SNO Collaboration) Phys. Rev. Letts. 87, 071301 (2001). 


\section{Eric Smith \\ Pacific Northwest National Laboratory}

\section{Education}

October 1998 Ph.D. University of Michigan, Nuclear Engineering and Radiological Sciences

December 1995 M.S. University of Michigan, Nuclear Engineering and Radiological Sciences

June 1994 B.S. Oregon State University, Nuclear Engineering

\section{Research and Development Experience}

Pacific Northwest National Laboratory, Senior Staff Scientist, Radiological and Chemical Sciences Group (July 2001 - present)

- Multi-coincidence, multidimensional analysis methods for proliferation-monitoring samples;

- Verification of MOX fuel plutonium mass via direct and indirect nondestructive assay methods.

Argonne National Laboratory, Staff Nuclear Engineer, Measurements and Analysis Group Leader

(November 1998 - July 2001)

- Burnup credit reactivity measurements for spent fuel criticality safety;

- Neutron slowing-down-time spectrometry for fissile mass measurement of intact nuclear fuel;

- Development of a segmented gamma scanner for TRU waste characterization;

- Development of fast coincidence techniques for MC\&A and Safeguards measurements.

University of Michigan, Research Assistant (August 1995 - October 1998)

Doctoral Dissertation: Design, Modeling and Performance of a Hybrid Portable Gamma Camera. Masters Thesis Project: Design and Performance of a Multiple Pinhole Industrial Gamma Camera.

Teaching Assistant: Lab Instructor for graduate and undergraduate level nuclear measurements courses.

Argonne National Laboratory, summer intern (1992, 1993)

\section{Selected Publications}

- L. E. Smith, N.M. Abdurrahman, "Neutron Spectrometry for the Assay of High Fissile Content Spent Fuel," submitted to Nuclear Technology, July 2001.

- L.E. Smith, C. Chen, D.K. Wehe, Z. He, "Hybrid Collimation for Industrial Gamma-Ray Imaging: Combining Spatially Coded and Compton Aperture Data," Nuclear Instruments \& Methods in Physics Research, Section A 462, pp. 576-587, September 2001.

- L.E. Smith, Z. He, D.K. Wehe, G.F. Knoll, S.J. Wilderman, "Design and Modeling of the Hybrid Portable Gamma Camera System," IEEE Transactions on Nuclear Science, Vol. 45(3), pp. 963-969, February 1998.

- Z. He, L.E. Smith, D.K. Wehe, G.F. Knoll, "The CSPD-2 Gamma-Ray Imaging System," IEEE Transactions on Nuclear Science, Vol. 44(3), pp. 911-915, June 1997.

- L.E. Smith, Z. He, D.K. Wehe, G.F. Knoll, "Pulse Processing Electronics with Anticoincident Circuitry for a Multi-Channel Portable Gamma Camera," IEEE Nuclear Science Symposium Conference Record, November 1996.

- D. R. Wahlquist, L. E. Smith, "Cold Sample Handling Glovebox for the NRAD Reactor Pneumatic Transfer System," American Glovebox Society Conference Proceedings, May 2001.

- L. E. Smith, W. R. Mosby, R. T. Klann, R.N. Hill and S. E. Aumeier, "Spent Fuel Fissile Mass Verification at the ANL-West Radioactive Scrap and Waste Facility," Waste Management 2001 Conference Proceedings, March, 2001. 


\section{Robert C. Thompson \\ Pacific Northwest National Laboratory}

\section{Education}

1978 - 1989 South Dakota School of Mines and Technology, Rapid City, South Dakota Bachelor of Science, Electrical Engineering

1991 - $2000 \quad$ Washington State University, Richland, Washington

Various Courses-Computer Science, MBA courses (no degree)

1999 - Present Battelle-Management Skills Development Program

Battelle-specific management training

\section{Professional Experience}

1989 - Present Pacific Northwest National Laboratory, Richland, Washington

Technical Specialist 2 (1989-1992)

Technical Specialist 3 (1992-1994)

Technical Specialist 4 (1994-1996)

Research Engineer 3 (1996-1999)

Research Engineer 4 (1999-2001)

Technical Group Manager, Instrument Development Laboratory (2001-present)

Line management position, responsible for the operation of the EMSL IDL at PNNL. The IDL is the primary technical support organization for the science staff at EMSL (a DOE user facility), providing electronic design capabilities (DC-6 GHz), digital control and data acquisition systems and software development.

1986 - 1989 Institute of Atmospheric Sciences Rapid City, South Dakota

Research Programmer

1984 - 1989 (contracted to) Battelle Memorial Institute, PNNL Rapid City, South Dakota

Technician

I maintained and updated, as necessary, experimental apparatus for the Double Beta Decay experiment, located 4850' underground at Homestake Gold Mine, Lead, South Dakota.

\section{Awards, Patents, References And Publications}

R\&D 100 Award (1998): Radionuclide Aerosol Sampler/Analyzer

Patents: RASA Multi-strip sample head design, ARSA gas collection technology 


\section{Werner Tornow}

Department of Physics and Triangle Universities Nuclear Laboratory

Box 90308, Duke University, Durham, NC 27708-0308, USA

\section{Education}

Diploma Degree

1967, University of Tubingen

Dr.rer.nat.

1972, University of Tubingen

Dr.rer.nat.habil.

1979, University of Tubingen

Career/Employment

Assistant Professor 1985, Duke University

Research Associate Professor 1988, Duke University

Research Professor 1994, Duke University

Director of TUNL 1996, Duke University

Professor 1999, Duke University

\section{Specialization}

(i) main field

Experimental Few-Body Physics, especially polarization phenomena in neutron and gamma-ray induced reactions

(ii) other fields

Neutrino physics and double-beta decay

(iii) current research interest

gamma-ray induced reactions on deuterons, ${ }^{3} \mathrm{He}$ and ${ }^{4} \mathrm{He}$

\section{Honors, Awards, Fellowships, Membership of Professional Societies}

Dissertation Award, "Habilitation" Award

Fellow of the American Physical Society

Member of the German Physical Society

Member of the American Physical Society

\section{Publications (list selected publications on page 2 of curriculum vitae)}

-Number of papers in refereed journals: $\quad 120$

-Number of communications to scientific meetings: 190

\section{Recent selected publications}

- "Gamma-Ray Production in a Storage Ring Free-Electron Laser," V.N. Litvinenko, B. Burnham, M. Emamian, N. Hower, J.M.J. Madey, P. Morcombe, P.G. O'Shea, S.H. Park, R. Sachtschale, K.D. Straub, G. Swift, P. Wang, Y. Wu, R.S. Canon, C.R. Howell, N.R. Roberson, E.C. Schreiber, M. Spraker, W. Tornow, H.R. Weller, I.V. Pinayev, N.G. Gavrilov, M.G. Fedotov, G.N. Kulipanov, G.Y. Kurkin, S.F. Mikhailov, V.M. Popik, A.N. Skrinsky, .A. Vinokurov, B.E. Norum, A. Lumpkin, and B. Yang, Phys. Rev. Lett. 78469 (1997). 
- "Determination of the ${ }^{3} \mathrm{P}_{\mathrm{j}}$ Phase Shifts from Nucleon-Nucleon Data: A Critical Evaluation and a Surprising Result," T. Tornow and W. Tornow, Few-Body Syst. 261 (1999).

- "New Measurement of the ${ }^{1} \mathrm{~S}_{0}$ Neutron-Neutron Scattering Length Using the Neutron-Proton Scattering Length as a Standard," D.E. González Trotter, F. Salinas, Q. Chen, A.S. Crowell, W. Glöckle, C.R. Howell, C.D. Roper, D. Schmidt, I. Slaus, H. Tang, W. Tornow, R.L. Walter, H. Witala, and Z. Zhou, Phys. Rev. Lett. 833788 (1999).

- "Double Polarized Neutron-Proton Scattering and Meson-Exchange NucleonNucleon Potential Models," B.W. Raichle, C.R. Gould, D.G. Haase, M.L. Seely, J.R. Walston, W. Tornow, W.S. Wilburn, S.I. Penttila, and G.W. Hoffmann, Phys. Rev. Lett. 832711 (1999).

- "Some Highlights of NN and 3N Studies at TUNL," W. Tornow, Few-Body Syst. Suppl. 1241 (2000).

- "First Measurement of the Near-Threshold ${ }^{2} \mathrm{H}(\gamma, n) \mathrm{p}$ Analyzing Power using FreeElectron Laser Based Gamma-Ray Source," E.C. Schreiber, R.S. Canon, B.T. Crowley, C.R. Howell, J.H. Kelley, V.N. Litvinenko, S.O. Nelson, S.H. Park, I.V. Pinayev, R.M. Prior, K. Sabourov, M. Spraker, W. Tornow, H.R. Weller, Y. Wu, and E.A. Wulf, Phys. Rev. C61 061604 (2000).

- "Experimental Few-Nucleon Studies at Low and Intermediate Energies," W. Tornow, Nucl. Phys. A684 193c (2001). 


\author{
Ray Allen Warner \\ Pacific Northwest National Laboratory
}

\title{
Education
}

- Doctor of Philosophy in Physics from University of California at Davis

- Bachelor of Science in Engineering Physics from University of California at Berkeley

\section{Employment}

- Pacific Northwest National Laboratory (1977 - present)

Program Manager, National Security

Nuclear Explosion Monitoring program developed the fission-product detection technology for all U.S. sites in the Comprehensive Nuclear-Test-Ban Treaty International Monitoring System; Radiation Detection Technology program developed glass fibers for neutron detection, optically-stimulated luminescent dosimeters and readers, large germanium-detector arrays for aerial surveys, and other technologies for arms-control treaties and environmental monitoring applications; Tags and Seals program developed primarily non-nuclear technologies for the Strategic Arms Reduction Talks (START)

Technical Advisor, DOE Office of Arms Control Division of Systems and Technology (1988-1990)

Sole nuclear scientist in the division, responsible for the success of multiple technical developments for nonproliferation and arms control

Senior Research Scientist, Analytical and Nuclear Sciences

Lifetimes of previously unobserved radioactive nuclides; fission yields and beta-delayed neutron emission; single-atom detection using laser-induced photon bursts

- Michigan State University Cyclotron Laboratory (1969 - 1977)

Research Associate and Assistant Professor

Accelerator based gamma spectroscopy; the weak interaction in atomic nuclei; nuclear astrophysics; collective behavior in nuclei; residual interaction between nucleons; teaching physics and chemistry

- University of California, Davis (1962 - 1969)

Graduate Research Assistant, Crocker Nuclear Laboratory

Structure of atomic nuclei in states of high angular momentum

- United States Army (1961 - 1962)

Air Defense Artillery Battery Officer

Training officer and Nike-Hercules launcher platoon leader

\section{Professional Societies and Honors}

- American Physical Society

- American Association for the Advancement of Science

- Sigma Xi

- Tau Beta Pi

- National Defense Education Act (NDEA) Fellow

\section{Physical Review Letters and other Selected Publications}

- Nanosecond Timing Using a Plastic Scintillator in a Cyclotron Beam, R.A. Warner, G.L. Smith, R.M. Lieder, and J.E. Draper, Nuclear Instruments \& Methods 75, 149 (1969) 
- Ground-State Quasi-rotational Bands to $8^{+}$in 114-126Te Produced by(a,xn), R.A. Warner and J.E. Draper, Physical Review C 1 , 1069 (1970).

- Collective and Higher-Order Effects Shown by the (p,t) Reaction on the Deformed Nucleus 159Tb, R.W. Goles, R.A. Warner, Wm. C. McHarris, and W.H. Kelly, Physical Review Letters 29, 802 (1972).

- Backbending and Forking in the Yrast States of Even Os Isotopes, R.A. Warner, F.M. Bernthal, J.S. Boyno, T.L. Khoo, and G. Sletten, Physical Review Letters 31, 835 (1973).

- An Experimental Demonstration of Backbending Behavior from a Band Crossing in 154Gd, T.L. Khoo, F.M. Bernthal, J.S. Boyno, and R.A. Warner, Physical Review Letters $\underline{31}, 1146$ (1973).

- Failure of the Allowed Assumption in the e/beta Decays of $145 \mathrm{gGd}$ and $143 \mathrm{gSm}$ Experimental Evidence for Interference Effects in Nuclear Beta Decay, R.B. Firestone, R.A. Warner, Wm. C. McHarris, and W.H. Kelly, Physical Review Letters 33, 30 (1974).

- Hexadecapole Deformations in W and Os Nuclei from Perturbed Rotational Band Structure, F.M. Bernthal, B.D. Jeltema, J.S. Boyno, T.L. Khoo, and R.A. Warner, Physical Review Letters $\underline{33}, 915$ (1974).

- Decoupled i13/2Neutrons and Backbending in W and Os Isotopes, F.M. Bernthal, J.S. Boyno, T.L. Khoo, and R.A. Warner, Physical Review Letters 33, 1313 (1974).

- Absolute Measurements of Anomalous e/beta Decay Branching Ratios, R. B. Firestone, R.A. Warner, Wm. C. McHarris, and W.H. Kelly, Physical Review Letters 35, 401 (1975).

- Residual Interactions in 4 - Quasiparticle $K^{\pi}=14$ - Isomer in 176Hf, T.L. Khoo, F.M. Bernthal, R.A. Warner, G.F. Bertsch, and G. Hamilton, Physical Review Letters 35, 1256 (1975).

- High-Spin Multiquasiparticle Yrast Traps in f 176Hf, T.L. Khoo, F.M. Bernthal, R. G. H. Robertson, and R. A. Warner, Physical Review Letters 37, 823 (1976).

- Measurement of the Internal Pair Emission Branch of the 7.654-MeV State of 12C, and the Rate of the Stellar Triple-alpha Reaction, R.G.H. Robertson, R.A. Warner, and Sam M. Austin, Physical Review C $\underline{15}, 1072$ (1977).

- Beta-Delayed Two-Neutron Emission from 98Rb, P.L. Reeder, R.A. Warner, T.R. Yeh, R.E. Chrien, R.L. Gill, M. Shmid, H.I. Liou, and M.L. Stelts, Physical Review Letters 47, 483 (1981).

- Observation of the Capture Reaction 2H(alpha, gamma)6Li and Its Role in Production of 6Li in the Big Bang, R.G.H. Robertson, P. Dyer, R.A. Warner, R.C. Melin, T.J. Bowles, A.B. McDonald, G.C. Ball, W.G. Davies, and E.D. Earle, Physical Review Letters $\underline{47}, 1867$ (1981).

- Method and Apparatus for Detecting Neutrons, R.W. Perkins, P.L. Reeder, N.A. Wogman, R.A. Warner, D.W. Brite, W.C. Richey, D.S. Goldman, U.S. Patent 5,680,423, Pacific Northwest National Laboratory, Richland, WA (1997).

- Description of DOE Radionuclide Aerosol Sampler/Analyzer for the Comprehensive Test Ban Treaty H.S. Miley, C.W. Hubbard, A.D. Mckinnon, R.W. Perkins, R.C. Thompson, and R.A. Warner, Journal of Radioanalytical and Nuclear Chemistry 235, 83 (1998).

- Automated Separation and Measurement of Radioxenon for Monitoring a Comprehensive Test Ban Treaty, T. W. Bowyer, K.H. Abel, C.W. Hubbard, M.E. Panisko, R.C. Thompson, and R. A. Warner, Journal of Radioanalytical and Nuclear Chemistry (1999). 


\section{Joel Webb}

Carlsbad Environmental Monitoring \& Research Center, New Mexico State University

\section{Education}

Master of Science in Health Physics 1994

Department of Radiological Health Sciences, College of Veterinary Medicine

Colorado State University, Fort Collins, Colorado

Bachelor of Science in Range Ecology 1991

Department of Range Science, College of Natural Resources

Colorado State University, Fort Collins, Colorado

\section{Professional Membership, Service And Honors}

- American National Standards Institute: Internal Dosimetry Programs for Plutonium Exposure- Minimum Requirements, ANSI N 13.25: Working Group (appointed)

- Department of Energy Applied Health Physics Fellowship

- Department of Energy 'Q' Clearance (inactive)

- Department of Energy Radiological Worker Level II

- Health Physics Society

- Certified by the American Academy of Health Physics

\section{Work Experience}

Manager, Program Development / Internal Dosimetry and Radiation Safety Officer, Carlsbad Environmental Monitoring \& Research Center, New Mexico State University, Carlsbad, NM September 1995 to Present

Radiation Safety Officer

Manager of the Internal Dosimetry Group

Manager of Program Development

Internal Dosimetry, Kaiser -Hill LLC Rocky Flats, Golden, Colorado

August 1994 to September 1995

Master of Science Candidate, Colorado State University, Fort Collins, Colorado August 1992 to August 1994

Research Associate-Ecologist, Colorado State University, Fort Collins, Colorado April 1991 to August 1992

\section{Proposals Funded As Principle Investigator}

- Analytical and Scientific Support for the Los Alamos National Laboratory, Carlsbad Operations

- Actinide Chemistry and Repository Science Program, Los Alamos National Laboratory; award \$717,000; $2000-2002$

- Determination of Plutonium and Trace Metals in Various Biological and Environmental Media (with R. Arimoto), Lovelace Respirator Research Institute; award \$12,100; 2000 - 
2001

- Determination of Uranium and Trace Metals in Hair and Urine via ICP-MS (with R. Arimoto), Freeborne \& Peters; award \$8000; 2000 - 2001

- In Vivo Radiobioassay Measurements, Waste Control Specialists, Inc; award \$233,916; $1998-2002$

- In Vivo Radiobioassay Measurements of WIPP Personnel, Westinghouse Electric Company; award \$457,554; 1998 - 2002

- Internal Dose Assessments from Historical Radiation Worker Records, MJW Corporation; $\$ 10,000 ; 1999-2000$

- Radiobioassays for Ir-192, Radiographic Specialists; award \$3,742; 1998.

- In Vivo Measurements of Radiation Workers at Pantex Plant, Mason Hanger Corporation, award $\$ 5,356 ; 1998$.

- In Vivo Measurements of Affected Workers at Hanford Site, Fluor Daniel Hanford; award $\$ 8,790 ; 1998-1999$.

- The Cow Counter: Technology for the Measure of Radio-Contaminates and Fat-Free Lean Content in Livestock, DOE via the Waste-Management Education and Research Consortium; award \$169,860; 1999 - 2000.

\section{Select Publications}

- Arimoto R., Nottingham, A. S., Webb, J. L., Schloesslin, C. A. Davis, D. D. Non-sea salt an other aerosol constituents at the South Pole during ISCAT, Geophysical Research Letters, Vol. 28, No. 19, pp. 3645 - 3648 (2001).

- Kirchner, T. B., Webb, J. L., Webb, S. B., Arimoto, R., Schoep, D. A. and Stewart, B. D. Variability in background levels of surface soil radionuclides in the vicinity of the waste isolation pilot plant, Journal of Environmental Radioactivity (in press, revised manuscript accepted 4/01).

- Webb, J. L., and Kramer G. H. An evaluation of germanium detectors employed for the measurement of radionuclides deposited in lungs using an experimental and monte carlo approach, Health Physics (in press, revised manuscript accepted 3/01).

- Kramer, G. H., Lopez, M. A., and Webb, J. L. A joint HML-CIEMAT-CEMRC project: testing a function to fit counting efficiency of a lung counting germanium detector array to muscle equivalent chest wall thickness and photon energy using a realistic torso phantom, Radiation Protection Dosimetry, Vol. 92, No. 4, pp. 323-327 (2000).

- Webb, J. L., Gadd, M., Bronsen, F., and Tench, O. An Evaluation of recent Lung Counting Technology, Radiation Protection Dosimetry, Vol. 89, Nos 3-4, pp. 183-191 (2000).

- Webb, J. L. and Kirchner, T. In Vivo Measurements of Residents in the Carlsbad, New Mexico Area, Radiation Protection Dosimetry, Vol. 89, Nos 3-4, pp. 325-332 (2000).

- Lee, S. C.; Orlandini, K. A.; Webb, J. L.; Schoep, D., Kirchner, T. Measurement of Baseline Atmosperic Plutonium-239,240 and Americium-241 in the Vicinity of the Waste Isolation Pilot Plant. Journal of Radioanalytical and Nuclear Chemistry Vol. 234, Nos 1-2 267-272 (1998). 
John F. Wilkerson

University of Washington

\section{A. Professional Preparation}

University of North Carolina, Chapel Hill

University of North Carolina, Chapel Hill

University of North Carolina, Chapel Hill

Los Alamos National Laboratory
Physics

Physics

Physics

Thesis: Isospin Mixing in

Light Nuclei

Postdoctoral Research Fellow
B.S. 1977

M.S. 1979

Ph.D. 1982

$1982-85$

\section{B. Appointments}

Associate Chair, Physics Department, University of Washington, 1999-2001

Professor of Physics, University of Washington, 1994 - present

Staff Member, Los Alamos National Laboratory, 1985 - 1994

\section{Publications}

Most closely related to proposed project:

"Measurement of the Rate of $v_{\mathrm{e}}+\mathrm{d}-->\mathrm{p}+\mathrm{p}+\mathrm{e}^{-}$Interactions Produced by ${ }^{8} \mathrm{~B}$ Solar Neutrinos at the Sudbury Neutrino Observatory”, Phys. Rev. Lett. 87071301 (2001) (with Q.R. Ahmad et al.)

"Neutrino", J.F. Wilkerson, McGraw-Hill Encyclopedia of Science and Technology, Eighth Edition (1997).

"Measurement of the solar neutrino capture rate by SAGE and implications for neutrino oscillations in vacuum", Phys. Rev. Lett. 834686 (1999) (with J.N. Abdurashitov et al.).

"The Russian-American Gallium Experiment (SAGE) Cr-neutrino Source Measurement”, Phys. Rev. Lett. 77, 4708, (1996). (with J.N. Abdurashitov et al.).

"Low-background ${ }^{3} \mathrm{He}$ proportional counters for use in the Sudbury Neutrino Observatory", IEEE Trans. Nucl. Sci. 46, 873 (1999) (with," M.C. Browne et al.).

Other significant publications:

"Limit on Electron Antineutrino Mass from Observation of the Beta Decay of Molecular Tritium", Phys. Rev. Lett. 67, 957 (1991) (with R.G.H. Robertson et al.).

"New limit on the D coefficient in polarized neutron decay", Phys Rev C62, 055501 (2000) (with L. J. Lising, et al.).

“Solar Fusion Cross Sections," Rev. Mod. Phys., 70, 1265 (1998) (with E.G. Adelberger et al.).

"Correspondence of Electron Spectra from Photoionization and Nuclear Internal Conversion", Phys. Rev. Lett. 67, 2291 (1991) (with D.L. Wark et al.).

“Isospin-Nonconserving Particle Decays in Light Nuclei”, Nuclear Physics A549 223 (1992) (with T.M. Mooney et al.).

\section{Synergistic Activities}

- As University of Washington Physics Department Associate Chair for Graduate Affairs, directed and oversaw the department's graduate student program 1999-2001.

- Regularly mentor high school students from around the Puget Sound area. Inaugural participant in the Los Alamos National Laboratory high school outreach program, overseeing numerous high school students. 
- Assist with the University of Washington Physics Research Experiences for Undergraduates program. In 1985, initiated summer program of hiring collage undergraduates into the Physics Division Sub-atomic Physics Group.

- One of initiators of the Washington Large Cosmic Ray Timing Array (WALTA) outreach project at the University of Washington. The program's goal is enhanced science education for teachers and students in the Seattle area by encouraging direct participation and collaboration in real science experiments.

- Presented lectures on "Experimental Neutrino Physics" at TASI Summer School, July 2000, on "Solar Neutrinos" at the SLAC Summer Institute Summer School, August 2000, "Weak Interactions in Nuclear Physics" at the National Summer School in Nuclear Physics, June 1993, on "Neutrino Physics" at the National Summer School in Nuclear Physics, June 2001.

\section{E. Collaborators and Other Affiliations}

Collaborators:

emiT: B. K. Fujikawa, S. J. Freedman (Univ. of California-Berkeley/LBNL); G. L. Jones (Hamilton College); T. J. Bowles, G. L. Greene (LANL); A. Garcia (Notre Dame), L. J. Lising (Univ. of Maryland); T. E. Chupp, K. P. Coulter (Univ. of Michigan); J. M. Adams, M. S. Dewey, J. S. Nico, A. K. Thompson, F. E. Wietfeldt (NIST); H. P. Mumm, R.G.H. Robertson (Univ. of Washington).

SAGE: J. N. Abdurashitov,V. N. Gavrin, V. V. Gorbachev, T. V. Ibragimova, A. V. Kalikhov, N. G. Khairnasov, T. V. Knodel S. V. Girin, I. N. Mirmov, A. A. Shikhin, E. P. Veretenkin, V. M. Vermul, V. E. Yants, G. T. Zatsepin (Institute for Nuclear Research); T. J. Bowles, W. A. Teasdale (LANL); M. L. Cherry (LSU); J. S. Nico (NIST); B. T. Cleveland, R. Davis, Jr., K. Lande (Univ. of Pennsylvania); S. R. Elliott (Univ. of Washington).

SNO: J. Boger, R.L. Hahn, M. Yeh (BNL); F. Dalnoki-Veress, J. Farine, D.R. Grant, C.K. Hargrove, I. Levine, K. McFarlane, A.T. Noble, D. Sinclair, M. Starinsky (Carleton Univ.); E.D. Hallman, S. Luoma, M.H. Schwendener, R. Tafirout, C.J. Virtue (Laurentian Univ.); Y.D. Chan, X. Chen, K.T. Lesko, A.D. Marino E.B. Norman, C.E. Okada, A.W.P. Poon, R.G. Stokstad (LBNL); T.J. Bowles, M.R. Dragowsky, A. Hamer, A. Hime, J.B. Wilhelmy, J.M. Wouters (LANL); S. Biller, M.G. Bowler, J. Cameron, B. Cleveland, X. Dai, G. Doucas, J. Dunmore, H. Fergani K. Frame, N.A. Jelley, S. Majerus, N. McCauley, G. McGregor, D.L. Wark, N. West, J. Wilson (Univ. of Oxford); M.G. Boulay, M. Chen, F.A. Duncan, E.D. Earle, H.C. Evans, G.T. Ewan, A.L. Hallin, P.J. Harvey, J.D. Hepburn, H.W. Lee, J.R. Leslie, H.B. Mak, A.B. MacDonald, B.A. Moffat, B.C. Robertson, P. Skensved (Queen's Univ.); J. Heise, R.L. Helmer, T. Kutter, C.W. Nally, C.E. Waltham (Univ. of British Columbia); P. Jagam, J. Law, I.T. Lawson, J.J. Simpson (Univ. of Guelph); E.W. Beier, D.F. Cowen, W. Frati, W.?. Heintzelman, P.T. Keener, J.R. Klein, C.C.M. Kyba, M.S. Neubauer, S.M. Oser, V.L. Rusu, R.G. Van de Water, R. Van Berg (Univ. of Pennsylvania); T.V. Bullard, T.H. Burritt, G.A. Cox, P.J. Doe, C.A. Duba, S.R. Elliott, A.A. Hamian, R. Hazama, K.M. Heeger, M. Howe, J.L. Orrell, R.G.H. Robertson, K.K. Schaffer, M.W.E. Smith (Univ. of Washington).

KATRIN: Guido Drexlin, Klaus Eitel, Markus Steidl, Hans Bluemer (Karlsruhe); Vladimir Lobashev (Institute for Nuclear Research); Jochen Bonn, Ernst Otten, Christian Weinheimer (Mainz); Alexander Osipowicz (Fuldu Univ.); Dragoun Otokar (Univ. of Prague); R.G.H. Robertson (Univ. of Washington).

NUSL: J. Conrad (Columbia), S. Farwell (SDSM\&T), W. Haxton (LANL), M. Marshak (Univ. of Minn.),

Graduate and Postgraduate Advisors:

Thesis Advisor: Edward J. Ludwig (UNC)

Postdoc Advisors: T.J. Bowles (LANL) and R.G.H. Robertson (Univ. of Washington)

Thesis Advisor and Postgraduate-Scholar Sponsor:

Students:

Michael C. Browne (Los Alamos National Laboratory), Eric G. Wasserman (Personify), Q. Rushdy Ahmad (Sapient, Corp.) Pieter H. Mumm (Univ. of Washington), J.L. Orrell (Univ. of Washington), Kareem Kazkaz (Univ. of Washington), G. Adam Cox (Univ. of Washington).

Postdocs:

D.L. Wark (Rutherford Laboratory/Univ of Sussex), William Hamilton (Oak Ridge National Laboratory), Steven R. Elliott (Univ. of Washington), Jeff S. Nico (NIST), J.V. Germani (WRQ Corp.), Reena MeijerDrees (Brooks Automation), T.D. Steiger (Cymer), Araz A. Hamian (Avocent Corporation), R. Hazama (Univ. of Washington), J.A. Formaggio (Univ. of Washington). 


\section{Albert R. Young \\ North Carolina State University}

\section{Academic Experience}

\begin{tabular}{lll} 
Position & Institution & Year \\
\hline Associate Professor & North Carolina State University & 2001 -present \\
Assistant Professor & North Carolina State University & $2000-2001$ \\
Assistant Professor & Princeton University & $1996-2000$ \\
Lecturer & Princeton University & $1994-1996$ \\
Research Associate & Princeton University & $1992-1994$ \\
Junior Research Fellow & California Institute of Technology & $1990-1992$ \\
Teaching Fellow & Harvard University & $1986-1987$ \\
Research Assistant & Harvard University & $1983-1990$ \\
Honors Tutor & University of Washington & $1979-1980$
\end{tabular}

\section{Education}

Harvard University -Ph.D. Physics (1990) \{Thesis Advisor: Dr. John Kohl

University of Washington - B. Sc. (1982)

Graduated with college and departmental honors (1982)

\section{Professional Societies}

American Physical Society: Division of Atomic, Molecular and Optical Physics, and Division of Nuclear Physics

American Association for the Advancement of Science

\section{Publications in Reverse Chronological Order}

"Ultra-Cold Neutron Upscattering in a Molecular Deuterium Crystal," C.-Y. Liu, A. R. Young and S. K. Lamoreaux, Rapid Communications: Phys. Rev. B 62, R3581 (2000).

"Depolarization of UCN Stored in Material Traps," A. Serebrov, A. Vasiliev, M. Lasakov, Yu. Rudnev, I. Krasnoshekova, P. Geltenbort, J. Butterworth, T. Bowles, C. Morris, S. Seestrom, D. Smith, A. R. Young, Nucl. Instr. and Meth. 440 , 717 (2000).

"Performance of the prototype LANL solid deuterium ultra-cold neutron source," R. E. Hill for the SD2 collaboration, with G. L. Greene, L. Marek, E. Pasyuk, A. Garcia, B. Fujikawa, S. Baessler, Nucl. Instr. and Meth. 440 , 674 (2000).

"Slow Spin Relaxation of Rb Atoms Con_ned in Glass Cells with Dense 4He Gas at 1.85 K," A. Hatakeyama, K. Oe, S. Hara, J. Arai, T. Yabuzaki, and A. R. Young, Phys. Rev. Lett. 84 , 1407 (2000).

"Polarization of 3He by spin exchange with optically pumped $\mathrm{Rb}$ and K vapors," A. Ben-Amar Baranga, S. Appelt, M. V. Romalis, C. J. Erickson, A. R. Young, G. D. Cates and W. Happer, Phys. Rev. Lett. 80 , 2801 (1998). 
"Search for Monoenergetic Positron Emission from Heavy-ion Collisions at Coulombbarrier Energies," the APEX collaboration, Phys. Rev. Lett 78 , 618 (1997).

"Dielectronic Recombination for C3+ in a Known External Field", D. W. Savin, L. D. Gardner, D. B. Reisenfeld, A. R. Young, and J. L. Kohl, Phys. Rev. A 53, 280 (1996).

"A Search for Narrow Sum-Energy Lines in Electron-Positron Pair Emission from Heavy-Ion Collisions Near the Coulomb Barrier," The APEX collaboration, Phys. Rev. Lett. 75,2658 (1995).

"Laser Oriented 36K for Time Reversal Symmetry Measurements," A.R. Young, W.S. Anderson, F.P. Calaprice, G.D. Cates, G.L. Jones, D.A. Krieger, R.B. Vogelaar, Rapid Communication: Phys. Rev. C 52, R464 (1995).

"Measurement of C3+ Dielectronic Recombination in a Known External Field," A. R. Young, L. D. Gardner, D. W. Savin, G. P. Lafyatis, A. Chutjian, S. Bliman, and J. L. Kohl, Phys. Rev. A 49, 357 (1993). 


\section{Current and Pending Support}

(See GPG Section II.D.8 for guidance on information to include on this form.)

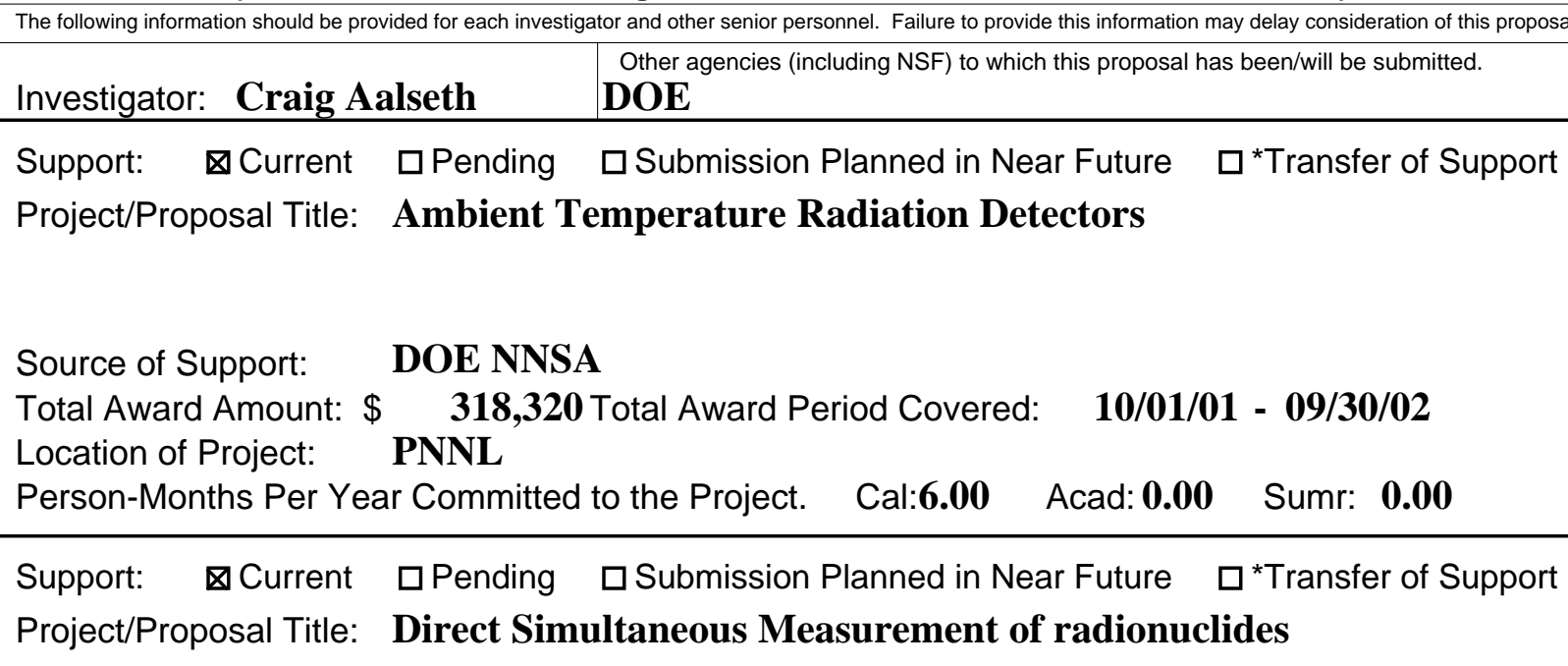

Source of Support: $\quad$ DOE NNSA

Total Award Amount: \$ 385,000 Total Award Period Covered: $\quad$ 10/01/01 - 09/30/02 Location of Project: PNNL

Person-Months Per Year Committed to the Project. Cal:6.00 Acad: 0.00 Sumr: 0.00

Support: $\quad$ Current $\square$ Pending $\square$ Submission Planned in Near Future $\square$ *Transfer of Support

Project/Proposal Title:

Source of Support:

Total Award Amount: $\$ \quad$ Total Award Period Covered:

Location of Project:

Person-Months Per Year Committed to the Project. Cal: Acad: Sumr:

Support: $\quad \square$ Current $\square$ Pending $\quad \square$ Submission Planned in Near Future $\quad{ }^{*}$ Transfer of Support

Project/Proposal Title:

Source of Support:

Total Award Amount: \$ Total Award Period Covered:

Location of Project:

Person-Months Per Year Committed to the Project. Cal: Acad: Sumr:

Support: $\quad \square$ Current $\square$ Pending $\square$ Submission Planned in Near Future $\quad{ }^{*}$ Transfer of Support

Project/Proposal Title:

Source of Support:

Total Award Amount: \$ Total Award Period Covered:

Location of Project:

Person-Months Per Year Committed to the Project. Cal: Acad: Summ:

*If this project has previously been funded by another agency, please list and furnish information for immediately preceding funding period.

NSF Form 1239 (10/99) Page G-2 USE ADDITIONAL SHEETS AS NECESSARY 


\section{Current and Pending Support}

(See GPG Section II.D.8 for guidance on information to include on this form.)

The following information should be provided for each investigator and other senior personnel. Failure to provide this information may delay consideration of this propos
Investigator: Dale Anderson

Source of Support: $\quad$ DOE

Total Award Amount: $\$ \quad$ 138,833 Total Award Period Covered: $\quad$ 10/01/01 - 09/30/02 Location of Project: PNNL

Person-Months Per Year Committed to the Project. Cal:9.00 Acad: 0.00 Sumr: 0.00

Support: $\square$ Current $\square$ Pending $\square$ Submission Planned in Near Future $\square{ }^{*}$ Transfer of Support

Project/Proposal Title:

Source of Support:

Total Award Amount: \$ Total Award Period Covered:

Location of Project:

Person-Months Per Year Committed to the Project. Cal: Acad: Sumr:

Support: $\square$ Current $\square$ Pending $\square$ Submission Planned in Near Future $\square$ *Transfer of Support

Project/Proposal Title:

Source of Support:

Total Award Amount: \$ Total Award Period Covered:

Location of Project:

Person-Months Per Year Committed to the Project. Cal: Acad: Sumr:

Support: $\quad \square$ Current $\square$ Pending $\quad \square$ Submission Planned in Near Future $\quad{ }^{*}$ Transfer of Support

Project/Proposal Title:

Source of Support:

Total Award Amount: \$ Total Award Period Covered:

Location of Project:

Person-Months Per Year Committed to the Project. Cal: Acad: Sumr:

Support: $\quad$ C Current $\square$ Pending $\square$ Submission Planned in Near Future $\quad{ }^{*}$ Transfer of Support

Project/Proposal Title:

Source of Support:

Total Award Amount: $\$ \quad$ Total Award Period Covered:

Location of Project:

Person-Months Per Year Committed to the Project. Cal: Acad: Summ:

${ }^{*}$ If this project has previously been funded by another agency, please list and furnish information for immediately preceding funding period.

NSF Form 1239 (10/99) Page G-3 USE ADDITIONAL SHEETS AS NECESSARY 


\section{Current and Pending Support}

(See GPG Section II.D.8 for guidance on information to include on this form.)

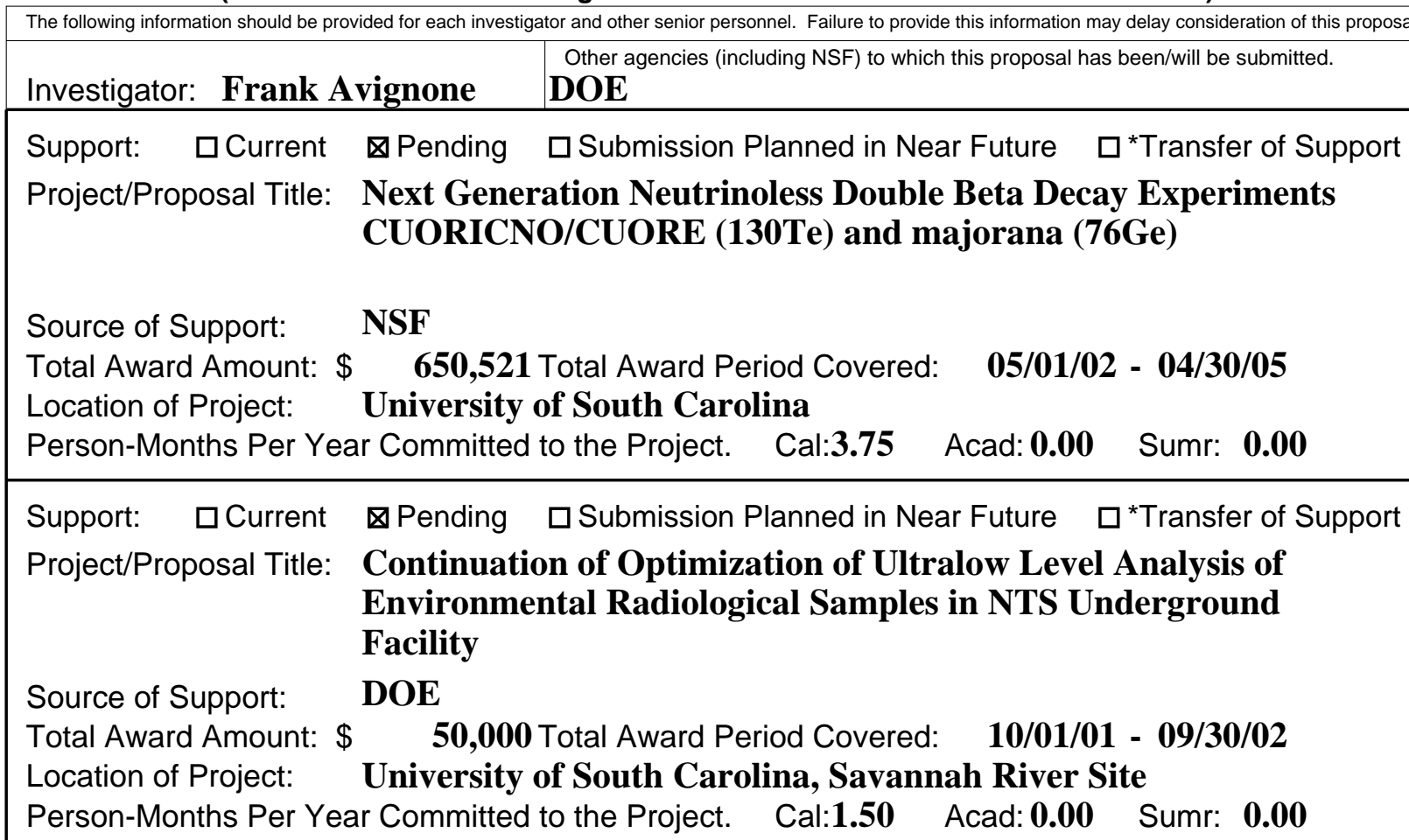

Support: $\quad$ Current $\square$ Pending $\square$ Submission Planned in Near Future $\square$ *Transfer of Support

Project/Proposal Title:

Source of Support:

Total Award Amount: $\$ \quad$ Total Award Period Covered:

Location of Project:

Person-Months Per Year Committed to the Project. Cal: Acad: Sumr:

Support: $\quad \square$ Current $\quad \square$ Pending $\quad \square$ Submission Planned in Near Future $\quad \square$ Transfer of Support

Project/Proposal Title:

Source of Support:

Total Award Amount: $\$ \quad$ Total Award Period Covered:

Location of Project:

Person-Months Per Year Committed to the Project. Cal: Acad: Sumr:

Support: $\quad \square$ Current $\quad \square$ Pending $\quad \square$ Submission Planned in Near Future $\quad \square$ *Transfer of Support

Project/Proposal Title:

Source of Support:

Total Award Amount: \$ Total Award Period Covered:

Location of Project:

Person-Months Per Year Committed to the Project. Cal: Acad: Summ:

*If this project has previously been funded by another agency, please list and furnish information for immediately preceding funding period.

NSF Form 1239 (10/99)

Page G-4

USE ADDITIONAL SHEETS AS NECESSARY 


\section{Current and Pending Support}

(See GPG Section II.D.8 for guidance on information to include on this form.)

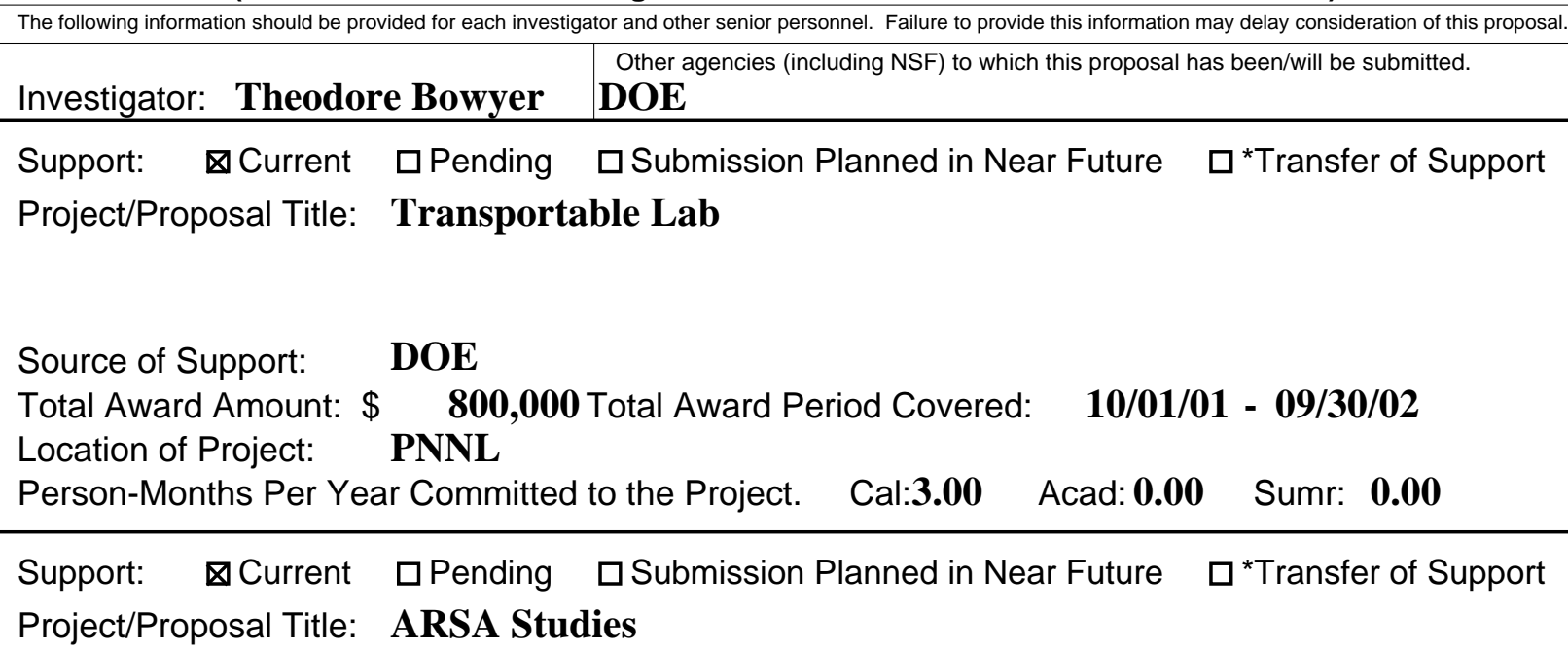

Source of Support: $\quad$ CTBTO

Total Award Amount: \$ 100,000 Total Award Period Covered: $\quad$ 10/01/01 - 09/30/02 Location of Project: PNNL

Person-Months Per Year Committed to the Project. Cal:2.00 Acad: 0.00 Sumr: 0.00

Support: $\quad$ Current $\square$ Pending $\square$ Submission Planned in Near Future $\square$ *Transfer of Support

Project/Proposal Title:

Source of Support:

Total Award Amount: \$ Total Award Period Covered:

Location of Project:

Person-Months Per Year Committed to the Project. Cal: Acad: Sumr:

Support: $\quad \square$ Current $\square$ Pending $\quad \square$ Submission Planned in Near Future $\quad{ }^{*}$ Transfer of Support

Project/Proposal Title:

Source of Support:

Total Award Amount: $\$ \quad$ Total Award Period Covered:

Location of Project:

Person-Months Per Year Committed to the Project. Cal: Acad: Sumr:

Support: $\quad$ Current $\square$ Pending $\square$ Submission Planned in Near Future $\quad{ }^{*}$ Transfer of Support

Project/Proposal Title:

Source of Support:

Total Award Amount: \$ Total Award Period Covered:

Location of Project:

Person-Months Per Year Committed to the Project. Cal: Acad: Summ:

*If this project has previously been funded by another agency, please list and furnish information for immediately preceding funding period.

NSF Form 1239 (10/99) $\quad$ Page G-5 USE ADDITIONAL SHEETS AS NECESSARY 


\section{Current and Pending Support}

(See GPG Section II.D.8 for guidance on information to include on this form.)

\begin{tabular}{|c|c|}
\hline Investigator: Ronald Brodzinski & $\begin{array}{l}\text { Other agencies (including NSF) to which this proposal has been/will be submitted. } \\
\text { DOE }\end{array}$ \\
\hline $\begin{array}{l}\text { Support: } \quad \text { \Current } \\
\text { Project/Proposal Title: }\end{array}$ & $\begin{array}{l}\square \text { Submission Planned in Near Future } \quad \square^{*} \text { Transfer of Support } \\
\text { iver Site Process Flow }\end{array}$ \\
\hline 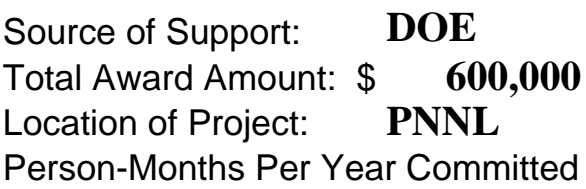 & $\begin{array}{l}\text { Total Award Period Covered: } \quad 10 / 01 / 01-09 / 30 / 02 \\
\text { to the Project. } \quad \text { Cal:7.00 } \quad \text { Acad: } 0.00 \quad \text { Sumr: } \mathbf{0 . 0 0}\end{array}$ \\
\hline
\end{tabular}

Source of Support: $\quad$ DOE

Total Award Amount: $\$ \quad \mathbf{7 0 0 , 0 0 0}$ Total Award Period Covered: $\quad$ 10/01/01 - 09/30/02 Location of Project: $\quad$ PNNL

Person-Months Per Year Committed to the Project. Cal:4.00 Acad: 0.00 Sumr: 0.00

Support: $\quad$ Current $\square$ Pending $\square$ Submission Planned in Near Future $\square$ *Transfer of Support

Project/Proposal Title:

Source of Support:

Total Award Amount: \$ Total Award Period Covered:

Location of Project:

Person-Months Per Year Committed to the Project. Cal: Acad: Sumr:

Support: $\quad \square$ Current $\square$ Pending $\quad \square$ Submission Planned in Near Future $\quad{ }^{*}$ Transfer of Support

Project/Proposal Title:

Source of Support:

Total Award Amount: \$ Total Award Period Covered:

Location of Project:

Person-Months Per Year Committed to the Project. Cal: Acad: Sumr:

Support: $\quad$ Current $\square$ Pending $\square$ Submission Planned in Near Future $\quad{ }^{*}$ Transfer of Support

Project/Proposal Title:

Source of Support:

Total Award Amount: \$ Total Award Period Covered:

Location of Project:

Person-Months Per Year Committed to the Project. Cal: Acad: Summ:

*If this project has previously been funded by another agency, please list and furnish information for immediately preceding funding period. NSF Form 1239 (10/99) Page G-6 USE ADDITIONAL SHEETS AS NECESSARY 


\section{Current and Pending Support}

(See GPG Section II.D.8 for guidance on information to include on this form.)

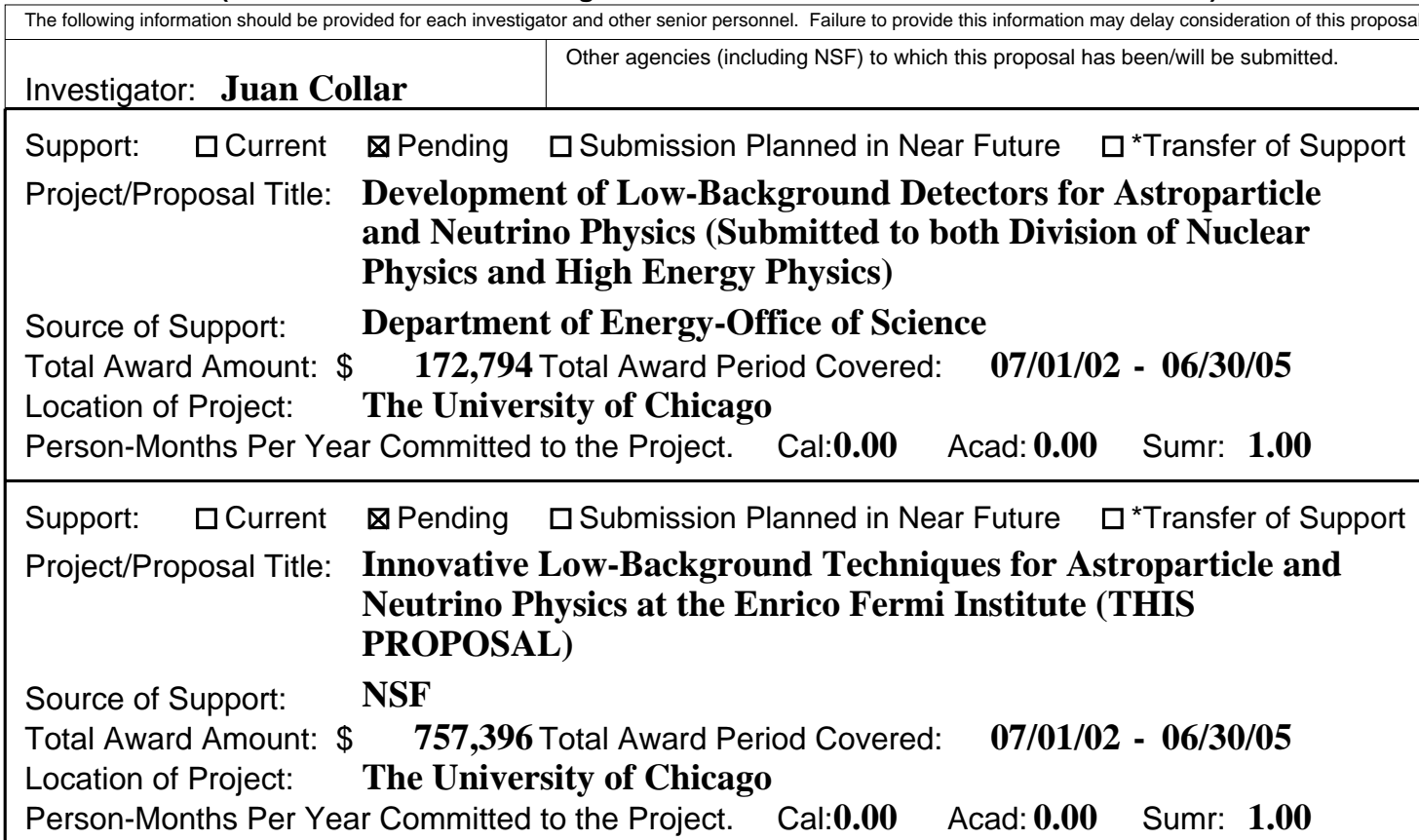

Support: $\square$ Current $\square$ Pending $\square$ Submission Planned in Near Future $\quad \square^{*}$ Transfer of Support

Project/Proposal Title:

Source of Support:

Total Award Amount: \$ Total Award Period Covered:

Location of Project:

Person-Months Per Year Committed to the Project. Cal: Acad: Sumr:

Support: $\quad$ Current $\square$ Pending $\square$ Submission Planned in Near Future $\square^{\star}$ Transfer of Support

Project/Proposal Title:

Source of Support:

Total Award Amount: \$ Total Award Period Covered:

Location of Project:

Person-Months Per Year Committed to the Project. Cal: Acad: Sumr:

Support: $\quad \square$ Current $\quad \square$ Pending $\quad \square$ Submission Planned in Near Future $\quad \square^{*}$ Transfer of Support

Project/Proposal Title:

Source of Support:

Total Award Amount: \$ Total Award Period Covered:

Location of Project:

Person-Months Per Year Committed to the Project. Cal: Acad: Summ:

*If this project has previously been funded by another agency, please list and furnish information for immediately preceding funding period. NSF Form 1239 (10/99) 


\section{Current and Pending Support}

(See GPG Section II.D.8 for guidance on information to include on this form.)

\begin{tabular}{|c|c|c|}
\hline The following information should be provided for each investiga & $\begin{array}{l}\text { ator and other senior personnel. Failure to provide this information } n \\
\text { Other agencies (including NSF) to which this proposal ha } \\
\text { DOE }\end{array}$ & $\begin{array}{l}\text { may delay consideration of this propo } \\
\text { has been/will be submitted. }\end{array}$ \\
\hline $\begin{array}{l}\text { Support: } \quad \text { \Current } \\
\text { Project/Proposal Title: }\end{array}$ & $\begin{array}{l}\square \text { Submission Planned in Near Future } \\
\text { tal Nuclear Physics }\end{array}$ & $\square^{*}$ Transfer of Support \\
\hline 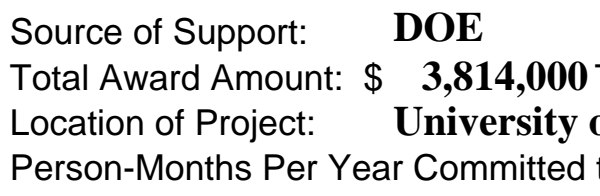 & $\begin{array}{l}\text { Total Award Period Covered: } \quad 12 / 01 / 0 \\
\text { of Washington } \\
\text { to the Project. Cal:12.00 Acad: } \mathbf{0 . 0 0}\end{array}$ & $\begin{array}{l}\text { 01 - 11/30/02 } \\
\text { Sumr: } 0.00\end{array}$ \\
\hline $\begin{array}{l}\text { Support: } \quad \square \text { Current } \quad \square \text { Pending } \\
\text { Project/Proposal Title: }\end{array}$ & $\square$ Submission Planned in Near Future & $\square *$ Transfer of Support \\
\hline $\begin{array}{l}\text { Source of Support: } \\
\text { Total Award Amount: } \$ \\
\text { Location of Project: } \\
\text { Person-Months Per Year Committed }\end{array}$ & $\begin{array}{l}\text { Total Award Period Covered: } \\
\text { to the Project. Cal: } \quad \text { Acad: }\end{array}$ & Sumr: \\
\hline $\begin{array}{l}\text { Support: } \quad \square \text { Current } \quad \square \text { Pending } \\
\text { Project/Proposal Title: }\end{array}$ & $\square$ Submission Planned in Near Future & $\square^{*}$ Transfer of Support \\
\hline $\begin{array}{l}\text { Source of Support: } \\
\text { Total Award Amount: } \$ \\
\text { Location of Project: } \\
\text { Person-Months Per Year Committed }\end{array}$ & $\begin{array}{l}\text { Total Award Period Covered: } \\
\text { to the Project. Cal: } \quad \text { Acad: }\end{array}$ & 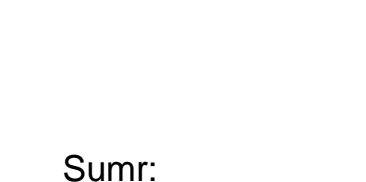 \\
\hline $\begin{array}{l}\text { Support: } \quad \square \text { Current } \quad \square \text { Pending } \\
\text { Project/Proposal Title: }\end{array}$ & $\square$ Submission Planned in Near Future & $\square *$ Transfer of Support \\
\hline $\begin{array}{l}\text { Source of Support: } \\
\text { Total Award Amount: } \$ \\
\text { Location of Project: } \\
\text { Person-Months Per Year Committed }\end{array}$ & $\begin{array}{l}\text { Total Award Period Covered: } \\
\text { to the Project. Cal: Acad: }\end{array}$ & Sumr: \\
\hline $\begin{array}{l}\text { Support: } \quad \square \text { Current } \quad \square \text { Pending } \\
\text { Project/Proposal Title: }\end{array}$ & $\square$ Submission Planned in Near Future & $\square *$ Transfer of Support \\
\hline $\begin{array}{l}\text { Source of Support: } \\
\text { Total Award Amount: } \$ \\
\text { Location of Project: } \\
\text { Person-Months Per Year Committed }\end{array}$ & $\begin{array}{l}\text { Total Award Period Covered: } \\
\text { to the Project. Cal: Acad: }\end{array}$ & Summ: \\
\hline
\end{tabular}




\section{Current and Pending Support}

(See GPG Section II.D.8 for guidance on information to include on this form.)

\begin{tabular}{|l|l|l|}
\hline The following information should be provided for each investigator and other senior personnel. Failure to provide this information may delay consideration of this proposal. \\
\hline $\begin{array}{l}\text { Investigator: Steve Elliott } \\
\text { DOE }\end{array}$
\end{tabular}

Source of Support: $\quad$ DOE

Total Award Amount: $\$ \quad 3,814,000$ Total Award Period Covered: $\quad$ 12/01/01 - 11/30/02

Location of Project: University of Washington

Person-Months Per Year Committed to the Project. Cal:10.30 Acad: 0.00 Sumr: 0.00

Support: $\quad$ Current $\square$ Pending $\square$ Submission Planned in Near Future $\square$ *Transfer of Support

Project/Proposal Title:

Source of Support:

Total Award Amount: $\$ \quad$ Total Award Period Covered:

Location of Project:

Person-Months Per Year Committed to the Project. Cal: Acad: Sumr:

Support: $\quad \square$ Current $\square$ Pending $\quad \square$ Submission Planned in Near Future $\quad{ }^{*}$ Transfer of Support

Project/Proposal Title:

Source of Support:

Total Award Amount: \$ Total Award Period Covered:

Location of Project:

Person-Months Per Year Committed to the Project. Cal: Acad: Sumr:

Support: $\quad$ Current $\square$ Pending $\square$ Submission Planned in Near Future $\quad{ }^{*}$ Transfer of Support

Project/Proposal Title:

Source of Support:

Total Award Amount: \$ Total Award Period Covered:

Location of Project:

Person-Months Per Year Committed to the Project. Cal: Acad: Summ:

*If this project has previously been funded by another agency, please list and furnish information for immediately preceding funding period. NSF Form 1239 (10/99) $\quad$ Page G-8 USE ADDITIONAL SHEETS AS NECESSARY 


\section{Current and Pending Support}

(See GPG Section II.D.8 for guidance on information to include on this form.)

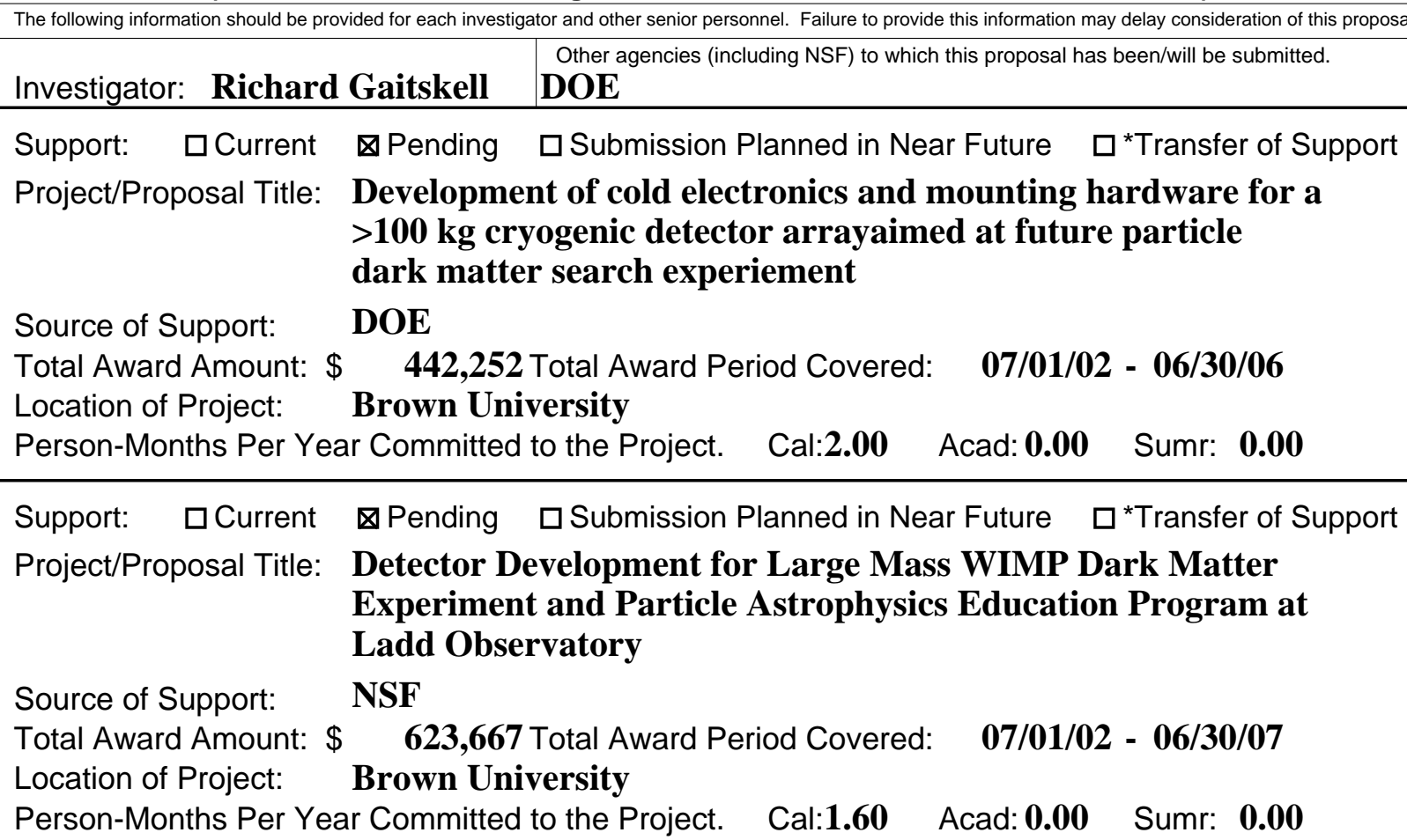

Support: $\quad \square$ Current $\quad \boldsymbol{\text { Pending }} \quad \square$ Submission Planned in Near Future $\quad{ }^{*}$ Transfer of Support

Project/Proposal Title: $\mathbf{X E N O N}$

Source of Support: $\quad$ NSF

Total Award Amount: $\$ \quad \mathbf{1 2 0 , 0 0 0}$ Total Award Period Covered: $\quad$ 07/01/02 - 06/30/04 Location of Project: Brown University

Person-Months Per Year Committed to the Project. Cal:0.00 Acad: 1.00 Sumr: $\mathbf{0 . 0 0}$

Support: $\quad \square$ Current $\square$ Pending $\quad \square$ Submission Planned in Near Future $\quad \square$ *Transfer of Support

Project/Proposal Title:

Source of Support:

Total Award Amount: \$ Total Award Period Covered:

Location of Project:

Person-Months Per Year Committed to the Project. Cal: Acad: Sumr:

Support: $\quad \square$ Current $\quad \square$ Pending $\quad \square$ Submission Planned in Near Future $\quad \square$ *Transfer of Support

Project/Proposal Title:

Source of Support:

Total Award Amount: \$ Total Award Period Covered:

Location of Project:

Person-Months Per Year Committed to the Project. Cal: Acad: Summ:

*If this project has previously been funded by another agency, please list and furnish information for immediately preceding funding period.

NSF Form 1239 (10/99)

Page G-9

USE ADDITIONAL SHEETS AS NECESSARY 


\section{Current and Pending Support}

(See GPG Section II.D.8 for guidance on information to include on this form.)

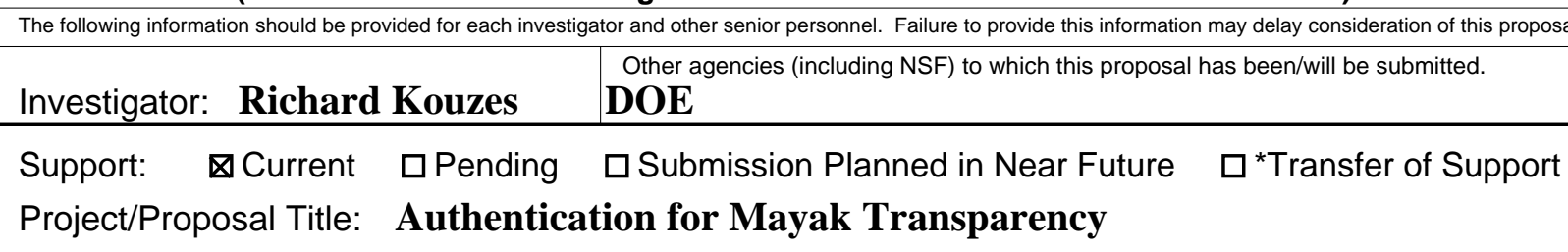

Source of Support: $\quad$ DoD DTRA

Total Award Amount: \$ 4,010,000 Total Award Period Covered: $\quad$ 09/01/00 - 09/30/02 Location of Project: Richland, WA

Person-Months Per Year Committed to the Project. Cal:9.00 Acad: 0.00 Sumr: 0.00

Support: $\square$ Current $\square$ Pending $\square$ Submission Planned in Near Future $\square{ }^{*}$ Transfer of Support

Project/Proposal Title:

Source of Support:

Total Award Amount: \$ Total Award Period Covered:

Location of Project:

Person-Months Per Year Committed to the Project. Cal: Acad: Sumr:

Support: $\square$ Current $\square$ Pending $\square$ Submission Planned in Near Future $\square$ *Transfer of Support

Project/Proposal Title:

Source of Support:

Total Award Amount: \$ Total Award Period Covered:

Location of Project:

Person-Months Per Year Committed to the Project. Cal: Acad: Sumr:

Support: $\quad \square$ Current $\square$ Pending $\square$ Submission Planned in Near Future $\quad{ }^{*}$ Transfer of Support

Project/Proposal Title:

Source of Support:

Total Award Amount: $\$ \quad$ Total Award Period Covered:

Location of Project:

Person-Months Per Year Committed to the Project. Cal: Acad: Sumr:

Support: $\quad$ Current $\square$ Pending $\square$ Submission Planned in Near Future $\quad{ }^{*}$ Transfer of Support

Project/Proposal Title:

Source of Support:

Total Award Amount: \$ Total Award Period Covered:

Location of Project:

Person-Months Per Year Committed to the Project. Cal: Acad: Summ:

${ }^{*}$ If this project has previously been funded by another agency, please list and furnish information for immediately preceding funding period.

NSF Form 1239 (10/99) Page G-1 USE ADDITIONAL SHEETS AS NECESSARY 


\section{Current and Pending Support}

(See GPG Section II.D.8 for guidance on information to include on this form.)

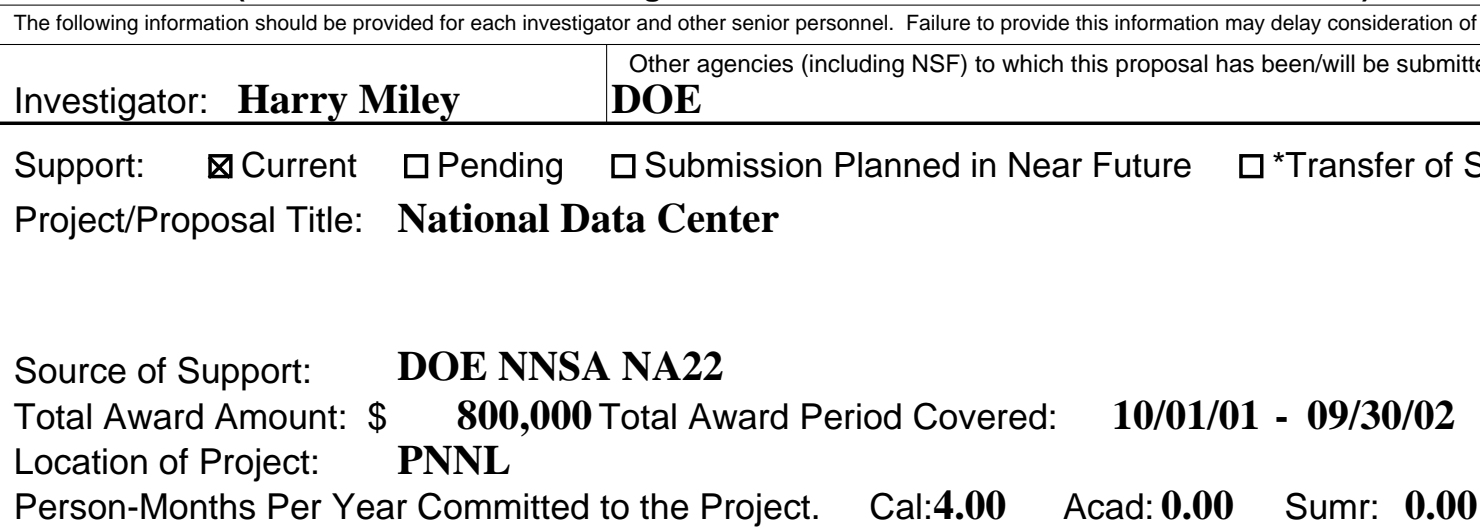

Support: $\quad$ Current $\square$ Pending $\square$ Submission Planned in Near Future $\square *$ Transfer of Support

Project/Proposal Title: Transportable Aerosol

Source of Support: $\quad$ DOE NNSA NA22

Total Award Amount: $\$ \quad$ 250,000 Total Award Period Covered: $\quad$ 10/01/01 - 09/30/02 Location of Project: $\quad$ PNNL

Person-Months Per Year Committed to the Project. Cal:4.00 Acad: 0.00 Sumr: 0.00

Support: $\quad$ Current $\quad \square$ Pending $\quad \square$ Submission Planned in Near Future $\quad{ }^{*}$ Transfer of Support

Project/Proposal Title: International Monitoring System Policy Support

Source of Support: $\quad$ DOE NNSA NA241

Total Award Amount: $\$ \quad \mathbf{6 5 , 0 0 0}$ Total Award Period Covered: $\quad$ 10/01/01 - 09/20/02 Location of Project: $\quad$ PNNL, Vienna International Center

Person-Months Per Year Committed to the Project. Cal:2.00 Acad: 0.00 Sumr: 0.00

Support: $\quad$ Current $\square$ Pending $\quad \square$ Submission Planned in Near Future $\quad \square$ *Transfer of Support Project/Proposal Title: Dual Field Sensor

Source of Support: $\quad$ DOE NNSA NA22

Total Award Amount: $\$ \quad 385,000$ Total Award Period Covered: $\quad$ 10/01/01 - 09/30/02 Location of Project: $\quad$ PNNL

Person-Months Per Year Committed to the Project. Cal:0.50 Acad: 0.00 Sumr: 0.00

Support: $\quad$ Current $\square$ Pending $\quad \square$ Submission Planned in Near Future $\quad \square$ *Transfer of Support

Project/Proposal Title: Majorana LDRD

Source of Support: $\quad$ PNNL LDRD

Total Award Amount: $\$ \quad$ 100,000 Total Award Period Covered: $\quad$ 10/01/01 - 09/30/02 Location of Project: $\quad$ PNNL

Person-Months Per Year Committed to the Project. Cal:2.00 Acad: 0.00 Summ: 0.00

*If this project has previously been funded by another agency, please list and furnish information for immediately preceding funding period.

NSF Form 1239 (10/99)

Page G-10

USE ADDITIONAL SHEETS AS NECESSARY 


\section{Current and Pending Support}

(See GPG Section II.D.8 for guidance on information to include on this form.)

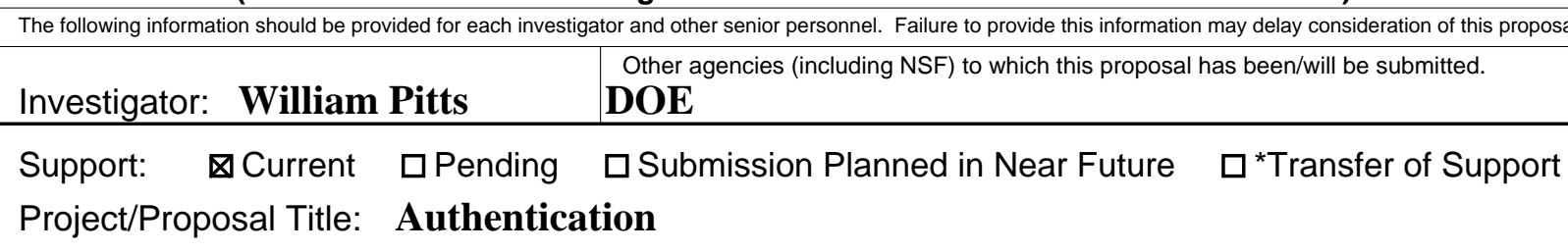

Source of Support: $\quad$ DOE DTRA

Total Award Amount: $\$$ 4,010,000 Total Award Period Covered: $\quad$ 09/01/00 - 09/30/02 Location of Project: PNNL

Person-Months Per Year Committed to the Project. Cal:9.00 Acad: 0.00 Sumr: 0.00

Support: $\quad$ C Current $\square$ Pending $\square$ Submission Planned in Near Future $\quad{ }^{*}$ Transfer of Support

Project/Proposal Title:

Source of Support:

Total Award Amount: \$ Total Award Period Covered:

Location of Project:

Person-Months Per Year Committed to the Project. Cal: Acad: Sumr:

Support: $\square$ Current $\square$ Pending $\square$ Submission Planned in Near Future $\square$ *Transfer of Support

Project/Proposal Title:

Source of Support:

Total Award Amount: \$ Total Award Period Covered:

Location of Project:

Person-Months Per Year Committed to the Project. Cal: Acad: Sumr:

Support: $\quad \square$ Current $\square$ Pending $\quad \square$ Submission Planned in Near Future $\quad{ }^{*}$ Transfer of Support

Project/Proposal Title:

Source of Support:

Total Award Amount: $\$ \quad$ Total Award Period Covered:

Location of Project:

Person-Months Per Year Committed to the Project. Cal: Acad: Sumr:

Support: $\quad$ Current $\square$ Pending $\square$ Submission Planned in Near Future $\quad{ }^{*}$ Transfer of Support

Project/Proposal Title:

Source of Support:

Total Award Amount: \$ Total Award Period Covered:

Location of Project:

Person-Months Per Year Committed to the Project. Cal: Acad: Summ:

${ }^{*}$ If this project has previously been funded by another agency, please list and furnish information for immediately preceding funding period.

NSF Form 1239 (10/99) Page G-11 USE ADDITIONAL SHEETS AS NECESSARY 


\section{Current and Pending Support}

(See GPG Section II.D.8 for guidance on information to include on this form.)

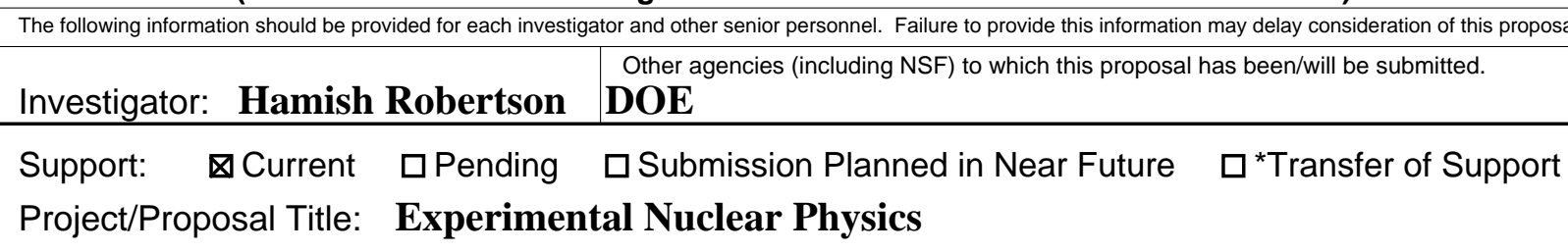

Source of Support: $\quad$ DOE

Total Award Amount: $\$$ 3,814,000 Total Award Period Covered: $\quad$ 12/01/01 - 11/30/02

Location of Project: University of Washington

Person-Months Per Year Committed to the Project. Cal:2.00 Acad: 0.00 Sumr: 0.00

Support: $\square$ Current $\square$ Pending $\square$ Submission Planned in Near Future $\square{ }^{*}$ Transfer of Support

Project/Proposal Title:

Source of Support:

Total Award Amount: \$ Total Award Period Covered:

Location of Project:

Person-Months Per Year Committed to the Project. Cal: Acad: Sumr:

Support: $\square$ Current $\square$ Pending $\square$ Submission Planned in Near Future $\square$ *Transfer of Support

Project/Proposal Title:

Source of Support:

Total Award Amount: \$ Total Award Period Covered:

Location of Project:

Person-Months Per Year Committed to the Project. Cal: Acad: Sumr:

Support: $\quad \square$ Current $\square$ Pending $\quad \square$ Submission Planned in Near Future $\quad{ }^{*}$ Transfer of Support

Project/Proposal Title:

Source of Support:

Total Award Amount: $\$ \quad$ Total Award Period Covered:

Location of Project:

Person-Months Per Year Committed to the Project. Cal: Acad: Sumr:

Support: $\quad$ C Current $\square$ Pending $\square$ Submission Planned in Near Future $\quad \square^{*}$ Transfer of Support

Project/Proposal Title:

Source of Support:

Total Award Amount: $\$ \quad$ Total Award Period Covered:

Location of Project:

Person-Months Per Year Committed to the Project. Cal: Acad: Summ:

*If this project has previously been funded by another agency, please list and furnish information for immediately preceding funding period.

NSF Form 1239 (10/99) $\quad$ Page G-12 USE ADDITIONAL SHEETS AS NECESSARY 


\section{Current and Pending Support}

(See GPG Section II.D.8 for guidance on information to include on this form.)

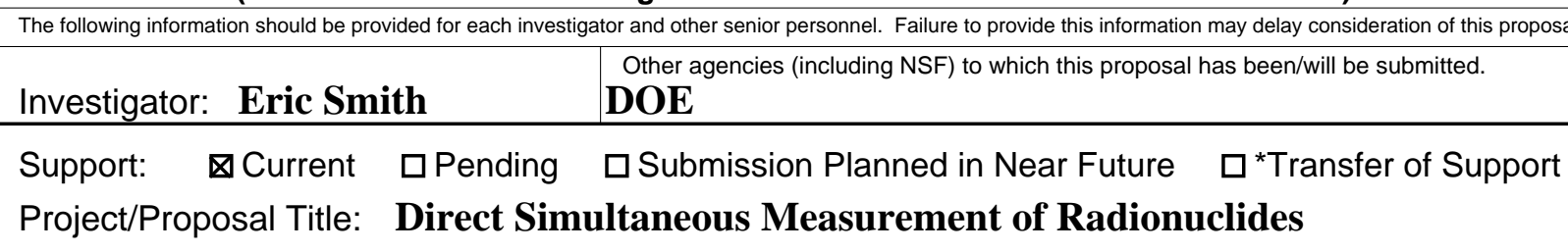

Source of Support: $\quad$ DOE NNSA

Total Award Amount: $\$ \quad \mathbf{3 8 5 , 0 0 0}$ Total Award Period Covered: $\quad$ 09/01/01 - 08/30/02 Location of Project: PNNL

Person-Months Per Year Committed to the Project. Cal:6.00 Acad: 0.00 Sumr: 0.00

Support: $\square$ Current $\square$ Pending $\square$ Submission Planned in Near Future $\square{ }^{*}$ Transfer of Support

Project/Proposal Title:

Source of Support:

Total Award Amount: \$ Total Award Period Covered:

Location of Project:

Person-Months Per Year Committed to the Project. Cal: Acad: Sumr:

Support: $\square$ Current $\square$ Pending $\square$ Submission Planned in Near Future $\square$ *Transfer of Support

Project/Proposal Title:

Source of Support:

Total Award Amount: \$ Total Award Period Covered:

Location of Project:

Person-Months Per Year Committed to the Project. Cal: Acad: Sumr:

Support: $\quad \square$ Current $\square$ Pending $\quad \square$ Submission Planned in Near Future $\quad{ }^{*}$ Transfer of Support

Project/Proposal Title:

Source of Support:

Total Award Amount: $\$ \quad$ Total Award Period Covered:

Location of Project:

Person-Months Per Year Committed to the Project. Cal: Acad: Sumr:

Support: $\quad$ Current $\square$ Pending $\square$ Submission Planned in Near Future $\quad{ }^{*}$ Transfer of Support

Project/Proposal Title:

Source of Support:

Total Award Amount: \$ Total Award Period Covered:

Location of Project:

Person-Months Per Year Committed to the Project. Cal: Acad: Summ:

*If this project has previously been funded by another agency, please list and furnish information for immediately preceding funding period.

NSF Form 1239 (10/99) Page G-13 USE ADDITIONAL SHEETS AS NECESSARY 


\section{Current and Pending Support}

(See GPG Section II.D.8 for guidance on information to include on this form.)

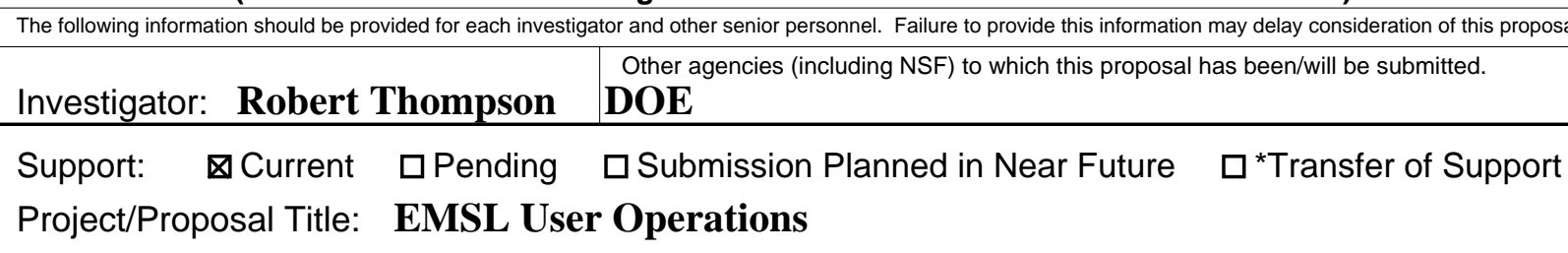

Source of Support: DOE OBER

Total Award Amount: $\$$ 1,190,000 Total Award Period Covered: $\quad$ 10/01/01 - 09/30/02 Location of Project: PNNL

Person-Months Per Year Committed to the Project. Cal:5.00 Acad: 0.00 Sumr: 0.00

Support: $\quad$ Current $\square$ Pending $\square$ Submission Planned in Near Future $\square{ }^{*}$ Transfer of Support

Project/Proposal Title: Electronics

Source of Support: $\quad$ DOE NNSA

Total Award Amount: $\$ \quad \mathbf{2 5 , 0 0 0}$ Total Award Period Covered: $\quad$ 10/01/01 - 09/30/02

Location of Project: PNNL

Person-Months Per Year Committed to the Project. Cal:0.50 Acad: 0.00 Sumr: $\mathbf{0 . 0 0}$

Support: $\quad$ Current $\square$ Pending $\square$ Submission Planned in Near Future $\quad{ }^{*}$ Transfer of Support

Project/Proposal Title:

Source of Support:

Total Award Amount: \$ Total Award Period Covered:

Location of Project:

Person-Months Per Year Committed to the Project. Cal: Acad: Sumr:

Support: $\quad \square$ Current $\square$ Pending $\square$ Submission Planned in Near Future $\quad{ }^{*}$ Transfer of Support

Project/Proposal Title:

Source of Support:

Total Award Amount: $\$ \quad$ Total Award Period Covered:

Location of Project:

Person-Months Per Year Committed to the Project. Cal: Acad: Sumr:

Support: $\square$ Current $\square$ Pending $\square$ Submission Planned in Near Future $\square{ }^{*}$ Transfer of Support

Project/Proposal Title:

Source of Support:

Total Award Amount: \$ Total Award Period Covered:

Location of Project:

Person-Months Per Year Committed to the Project. Cal: Acad: Summ:

*If this project has previously been funded by another agency, please list and furnish information for immediately preceding funding period.

NSF Form 1239 (10/99) Page G-14 USE ADDITIONAL SHEETS AS NECESSARY 


\section{Current and Pending Support}

(See GPG Section II.D.8 for guidance on information to include on this form.)

\begin{tabular}{|c|c|}
\hline Investigator: Werner Tornow & $\begin{array}{l}\text { Other agencies (including NSF) to which this proposal has been/will be submitted. } \\
\text { DOE }\end{array}$ \\
\hline $\begin{array}{ll}\text { Support: } \quad \boldsymbol{\otimes C u r r e n t} & \square \text { Pending } \\
\text { Project/Proposal Title: } & \text { Studies of } \\
& \text { particales }\end{array}$ & $\begin{array}{l}\square \text { Submission Planned in Near Future } \quad \square \text { *Transfer of Support } \\
\text { Nuclear Structure Using Neutrons and Charged }\end{array}$ \\
\hline $\begin{array}{l}\text { Source of Support: } \quad \text { DOE } \\
\text { Total Award Amount: } \mathbf{\$} \mathbf{2 , 5 5 0 , 0 0 0} \\
\text { Location of Project: } \quad \text { TUNL } \\
\text { Person-Months Per Year Committed }\end{array}$ & $\begin{array}{l}\text { Total Award Period Covered: } \quad 04 / 01 / 01-03 / 31 / 02 \\
\text { to the Project. Cal:8.00 Acad: } \mathbf{0 . 0 0} \quad \text { Sumr: } \mathbf{0 . 0 0}\end{array}$ \\
\hline
\end{tabular}

Source of Support: $\quad$ DOE

Total Award Amount: \$ 1,200,000 Total Award Period Covered: $\quad$ 08/01/01 - 03/31/02

Location of Project: Duke FEL Laboratory and TUNL

Person-Months Per Year Committed to the Project. Cal:1.30 Acad: 0.00 Sumr: 0.00

Support: $\quad \square$ Current $\quad \nabla$ Pending $\quad \square$ Submission Planned in Near Future $\quad \square{ }^{*}$ Transfer of Support

Project/Proposal Title: Unergraduate Research in Nuclear Physics at TUNL

Source of Support: $\quad$ NSF

Total Award Amount: $\$ \quad \mathbf{6 0 , 0 0 0}$ Total Award Period Covered: $\quad$ 05/01/01 - 08/31/02 Location of Project: TUNL

Person-Months Per Year Committed to the Project. Cal:0.70 Acad: 0.00 Sumr: 0.00

Support: $\quad \square$ Current $\square$ Pending $\quad \square$ Submission Planned in Near Future $\quad \square$ Transfer of Support

Project/Proposal Title:

Source of Support:

Total Award Amount: \$ Total Award Period Covered:

Location of Project:

Person-Months Per Year Committed to the Project. Cal: Acad: Sumr:

Support: $\quad$ Current $\square$ Pending $\square$ Submission Planned in Near Future $\quad{ }^{*}$ Transfer of Support

Project/Proposal Title:

Source of Support:

Total Award Amount: \$ Total Award Period Covered:

Location of Project:

Person-Months Per Year Committed to the Project. Cal: Acad: Summ:

*If this project has previously been funded by another agency, please list and furnish information for immediately preceding funding period.

NSF Form 1239 (10/99) Page G-15 USE ADDITIONAL SHEETS AS NECESSARY 


\section{Current and Pending Support}

(See GPG Section II.D.8 for guidance on information to include on this form.)

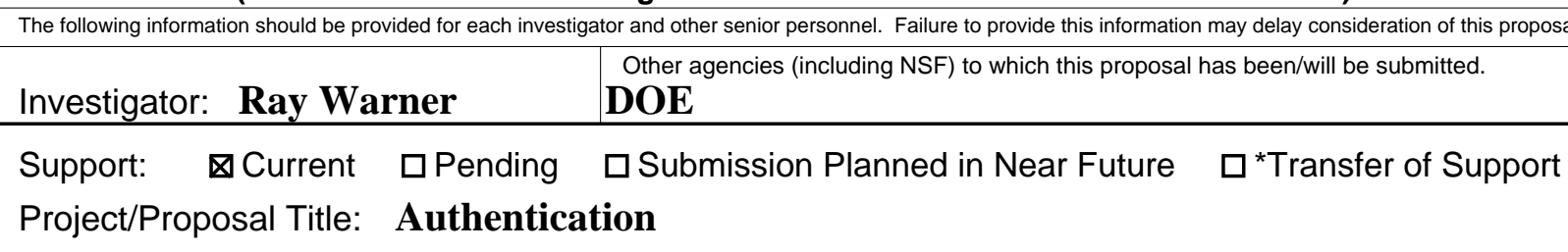

Source of Support: $\quad$ DoD DTRA

Total Award Amount: $\$$ 4,010,000 Total Award Period Covered: $\quad$ 09/01/00 - 09/30/02 Location of Project: PNNL

Person-Months Per Year Committed to the Project. Cal:6.00 Acad: 0.00 Sumr: 0.00

Support: $\square$ Current $\square$ Pending $\square$ Submission Planned in Near Future $\square{ }^{*}$ Transfer of Support

Project/Proposal Title:

Source of Support:

Total Award Amount: \$ Total Award Period Covered:

Location of Project:

Person-Months Per Year Committed to the Project. Cal: Acad: Sumr:

Support: $\square$ Current $\square$ Pending $\square$ Submission Planned in Near Future $\square$ *Transfer of Support

Project/Proposal Title:

Source of Support:

Total Award Amount: \$ Total Award Period Covered:

Location of Project:

Person-Months Per Year Committed to the Project. Cal: Acad: Sumr:

Support: $\quad \square$ Current $\square$ Pending $\square$ Submission Planned in Near Future $\quad{ }^{*}$ Transfer of Support

Project/Proposal Title:

Source of Support:

Total Award Amount: $\$ \quad$ Total Award Period Covered:

Location of Project:

Person-Months Per Year Committed to the Project. Cal: Acad: Sumr:

Support: $\quad$ Current $\square$ Pending $\square$ Submission Planned in Near Future $\quad{ }^{*}$ Transfer of Support

Project/Proposal Title:

Source of Support:

Total Award Amount: \$ Total Award Period Covered:

Location of Project:

Person-Months Per Year Committed to the Project. Cal: Acad: Summ:

*If this project has previously been funded by another agency, please list and furnish information for immediately preceding funding period.

NSF Form 1239 (10/99) Page G-16 USE ADDITIONAL SHEETS AS NECESSARY 


\section{Current and Pending Support}

(See GPG Section II.D.8 for guidance on information to include on this form.)

The following information should be provided for each investigator and other senior personnel. Failure to provide this information may delay consideration of this proposal.

\begin{tabular}{l|l}
\hline & Other agencies (including NSF) to which this proposal has been/will be
\end{tabular} Investigator: Joel L. Webb

DOE

Support: $\quad \square$ Current $\quad \square$ Pending $\quad \square$ Submission Planned in Near Future $\quad \square$ *Transfer of Support

Project/Proposal Title: Carlsbad Environmental Monitoring \& Research Program

Source of Support: DOE

Total Award Amount: $\$ 33,000,000$

Total Award Period Covered: 9/1/91 - 9/1/08

Location of Project: Carlsbad, New Mexico

Person-Months Per Year Committed to the Project. Cal: $5.0 \quad$ Acad: Sumr:

Support: $\square$ Current $\square$ Pending $\square$ Submission Planned in Near Future $\square$ *Transfer of Support

Project/Proposal Title: Actinide Chemistry and Repository Science Program

Source of Support: Los Alamos National Laboratory

Total Award Amount: $\$ 1,469,000 \quad$ Total Award Period Covered: 5/1/01 - 12/29/04

Location of Project: Carlsbad, New Mexico

Person-Months Per Year Committed to the Project. Cal: $3.0 \quad$ Acad: $\quad$ Sumr:

Support: $\quad \square$ Current $\square$ Pending $\quad \square$ Submission Planned in Near Future $\quad \square$ *Transfer of Support

Project/Proposal Title: In Vivo Radiobioassay Measurements

Source of Support: Waste Control Specialists

Total Award Amount: $\$ 233,916$

Total Award Period Covered: 7/1/98 - 7/1/02

Location of Project: Carlsbad, New Mexico

Person-Months Per Year Committed to the Project.

Cal: $1.0 \quad$ Acad:

Sumr:

Support: $\quad \square$ Current $\square$ Pending $\quad \square$ Submission Planned in Near Future $\quad \square$ *Transfer of Support

Project/Proposal Title: In Vivo Radiobioassay Measurements

Source of Support: Westinghouse True Solutions

Total Award Amount: $\$ 457,554 \quad$ Total Award Period Covered: 9/1/98 - 9/1/02

Location of Project: Carlsbad, New Mexico

Person-Months Per Year Committed to the Project.

Cal: $1.0 \quad$ Acad: $\quad$ Sumr:

Support: $\square$ Current $\quad \square$ Pending $\square$ Submission Planned in Near Future $\quad \square$ *Transfer of Support

Project/Proposal Title: Actinide Chemistry \& Repository Science Initiative

Source of Support: DOE

Total Award Amount: $\$ 7,072,767 \quad$ Total Award Period Covered: 9/1/01 - 9/1/08

Location of Project: Carlsbad, New Mexico

Person-Months Per Year Committed to the Project. Cal: $2.0 \quad$ Acad: $\quad$ Sumr:

*If this project has previously been funded by another agency, please list and furnish information for immediately preceding funding period.

NSF Form 1239 (10/99)

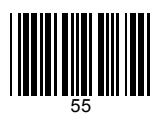




\section{Current and Pending Support}

(See GPG Section II.D.8 for guidance on information to include on this form.)

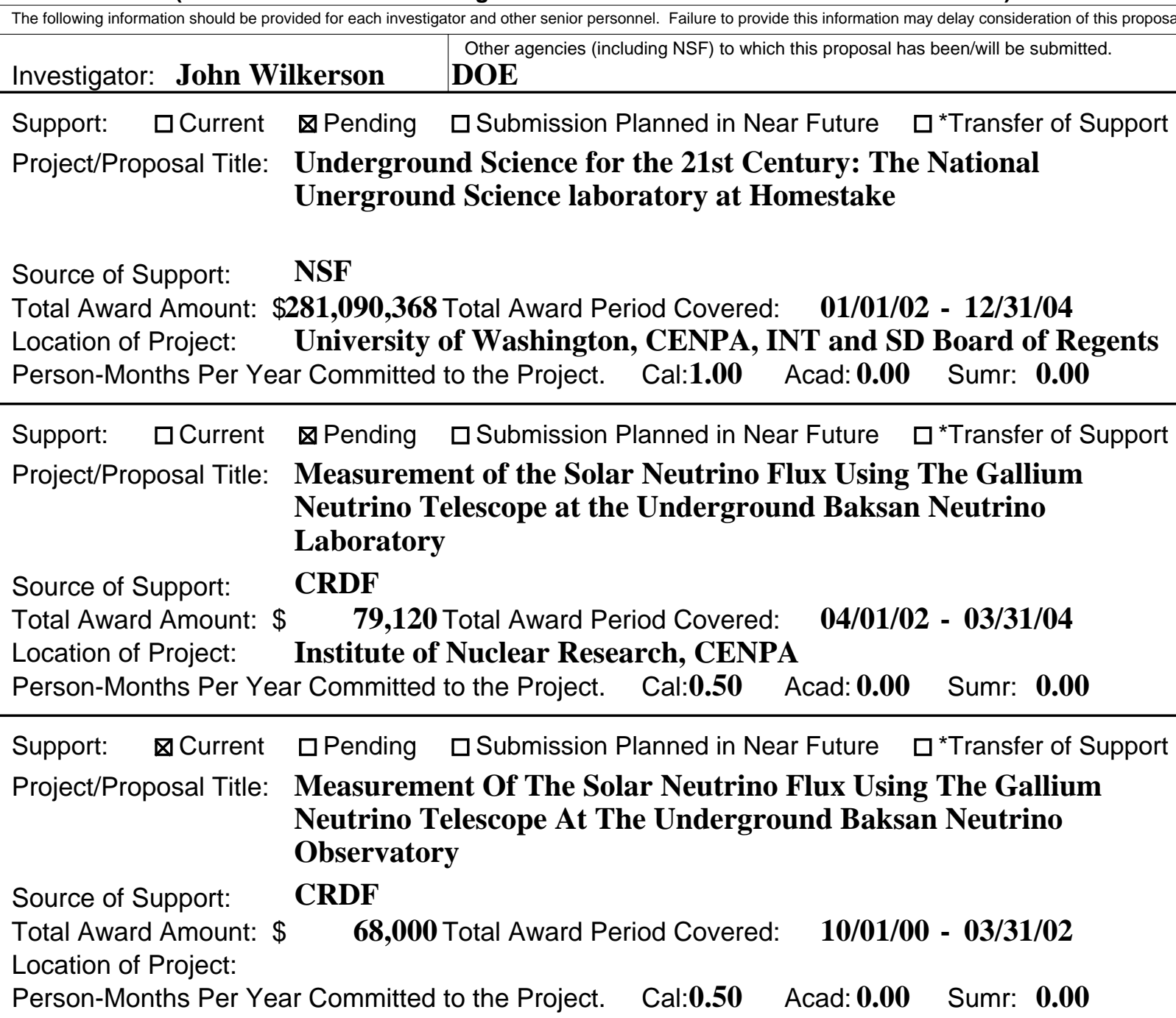

Support: $\quad$ Current $\square$ Pending $\quad \square$ Submission Planned in Near Future $\quad \square$ *Transfer of Support Project/Proposal Title: Experimental Nuclear Physics

Source of Support: $\quad$ DOE

Total Award Amount: $\$$ 3,814,000 Total Award Period Covered: 12/01/01 - 11/30/02 Location of Project: University of Washington

Person-Months Per Year Committed to the Project. Cal:2.00 Acad: 0.00 Sumr: 0.00

Support: $\quad \square$ Current $\quad \square$ Pending $\quad \square$ Submission Planned in Near Future $\quad \square^{*}$ Transfer of Support

Project/Proposal Title:

Source of Support:

Total Award Amount: $\$ \quad$ Total Award Period Covered:

Location of Project:

Person-Months Per Year Committed to the Project. Cal: Acad: Summ:

*If this project has previously been funded by another agency, please list and furnish information for immediately preceding funding period.

NSF Form 1239 (10/99)

Page G-17

USE ADDITIONAL SHEETS AS NECESSARY 


\section{Current and Pending Support}

(See GPG Section II.D.8 for guidance on information to include on this form.)

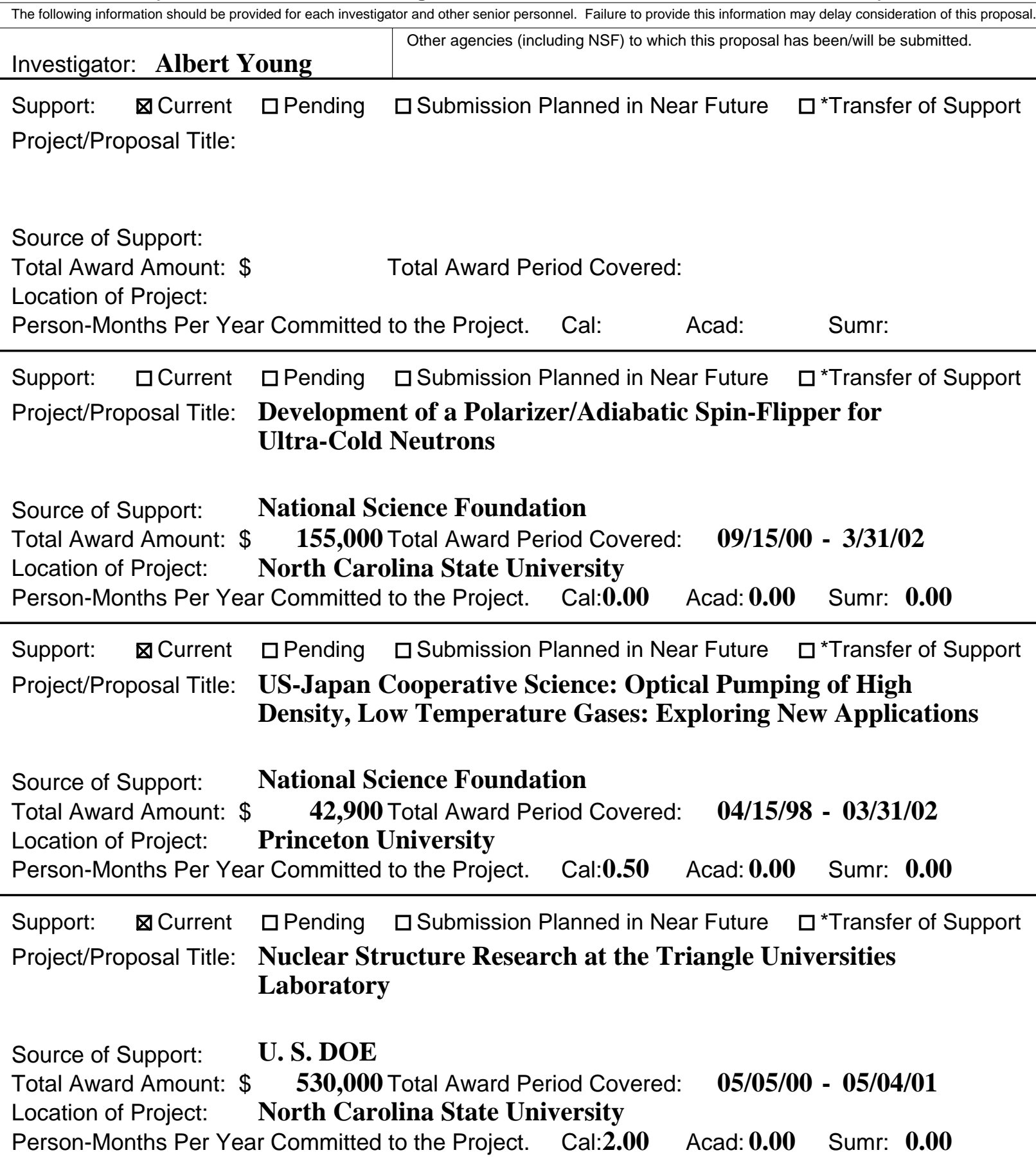

Person-Months Per Year Committed to the Project. Cal.2.00 Acad:0.00 Sumr: 0.00

$\begin{array}{ll}\text { Support: } \square \text { Current } & \boldsymbol{\otimes} \text { Pending } \square \text { Submission Planned in Near Future } \square{ }^{*} \text { Transfer of Support } \\ \text { Project/Proposal Title: } & \text { A Measurement of the Neutron Beta-Asymmetry Using Ultra-Cold } \\ & \text { Neutrons Produced in a Superthermal Solid Deuterium Source }\end{array}$

Source of Support: National Science Foundation

Total Award Amount: $\$ \quad$ 495,000 Total Award Period Covered: $\quad$ 07/01/01 - 06/30/04 Location of Project: North Carolina State University

Person-Months Per Year Committed to the Project. Cal:9.50 Acad: 0.00 Summ: 0.00

*If this project has previously been funded by another agency, please list and furnish information for immediately preceding funding period. NSF Form 1239 (10/99) Page G-1 USE ADDITIONAL SHEETS AS NECESSARY 